
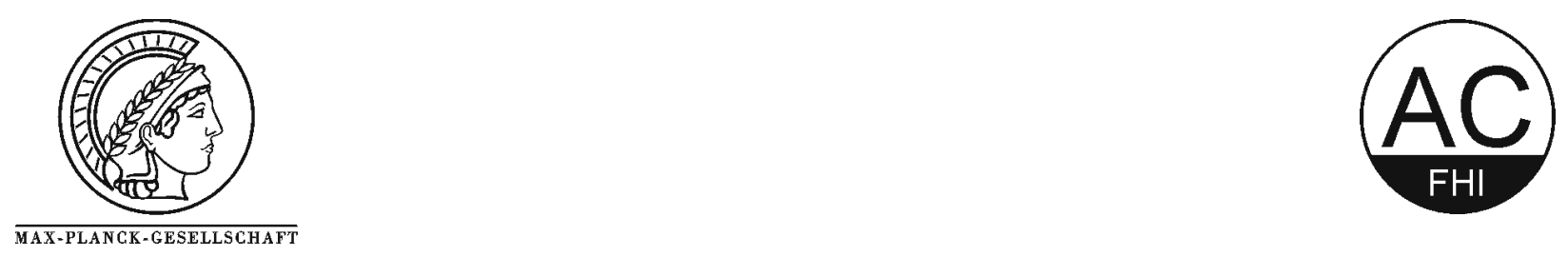

Published in: Progress in Surface Science 70 (2002) 1-151

\title{
Surface chemistry and catalysis on well-defined epitaxial iron-oxide layers
}

\author{
Werner Weiss and Wolfgang Ranke* \\ Department of Inorganic Chemistry, Fritz-Haber-Institut of the Max Planck Gesellschaft, \\ Faradayweg 4-6, 14195 Berlin, Germany \\ * Corresponding author: e-mail ressler@,fhi-berlin.mpg.de, phone +49 308413 4523, fax +49 \\ 3084134401
}

\begin{abstract}
Metal-oxide based catalysts are used for many important synthesis reactions in the chemical industry. A better understanding of the catalyst operation can be achieved by studying elemantary reaction steps on well-defined model catalyst systems. For the dehydrogenation of ethylbenzene to styrene in the presence of steam both unpromoted and potassium promoted iron-oxide catalysts are active. Here we review the work done over unpromoted single-crystalline $\mathrm{FeO}(111), \mathrm{Fe}_{3} \mathrm{O}_{4}(111)$ and $\alpha-\mathrm{Fe}_{2} \mathrm{O}_{3}(0001)$ films grown epitaxially on $\operatorname{Pt}(111)$ substrates. Their geometric and electronic surface structures were characterized by STM, LEED, electron microscopy and electron spectroscopic techniques. In an integrative approach, the interaction of water, ethylbenzene and styrene with these films was investigated mainly by thermal desorption and photoelectron emission spectroscopy. The adsorptiondesorption energetics and kinetics depend on the oxide surface terminations and are correlated to the electronic structures and acid-base properties of the corresponding oxide phases, which reveal insight into the nature of the active sites and into the catalytic function of semiconducting oxides in general. Catalytic studies, using a batch reactor arrangement at high gas pressures and post reaction surface analysis, showed that only $\alpha-\mathrm{Fe}_{2} \mathrm{O}_{3}(0001)$ containing surface defects is catalytically active, whereas $\mathrm{Fe}_{3} \mathrm{O}_{4}(111)$ is always inactive. This can be related to the elementary adsorption and desorption properties observed in ultrahigh vacuum, which indicates that the surface chemical properties of the iron-oxide films do not change significantly across the "pressure-gap". A model is proposed according to which the active site involves a regular acidic surface sites and a defect site next to it. The results on metal-oxide surface chemistry also have implications for other fields, such as environmental science, biophysics and chemical sensors.
\end{abstract}




\section{Contents}

1. Introduction

2. Basic concepts $\quad 6$

2.1. Adsorption process $\quad 6$

2.2. Thermodynamics and energetics of adsorption $\quad 10$

2.3. Langmuir isotherm 13

2.4. Kinetic analysis of isosteric heat of adsorption 14

$\begin{array}{ll}\text { 2.5. Sticking coefficients } & 15\end{array}$

2.6. Microkinetic modeling of surface reactions 17

2.7. Preparation of ordered metal-oxide surfaces 19

2.8. Energetics of metal-oxide surfaces 21

3. Experimental $\quad 23$

3.1. Instrumentation $\quad 23$

$\begin{array}{ll}\text { 3.1.1. Surface analysis systems } & 23\end{array}$

3.1.2. Sample transfer and heating $\quad 24$

3.1.3. High pressure reaction cells 25

3.2. Spectroscopic and micoscopic methods 25

3.2.1. STM, LEED, TDS and PEEM 25

3.2.2. XPS, UPS and XAS 26

3.3. Preparation of epitaxial iron-oxide films 26

$\begin{array}{ll}\text { 4. Styrene synthesis reaction } & 27\end{array}$

$\begin{array}{ll}\text { 4.1. Thermodynamics and byproducts } & 27\end{array}$

4.2. Technical catalysts $\quad 28$

4.3. Reaction kinetics and mechanism 29

4.4. Oxydehydrogenation of ethylbenzene over carbon-based catalysts 30

5. Growth and characterization of epitaxial iron-oxide films 3

5.1. Iron-oxide crystal structures and stability ranges 30

5.2. Identification of iron-oxide phases

5.2.1. LEED patterns 33

5.2.2. AES and XPS measurements $\quad 34$

$\begin{array}{ll}\text { 5.2.3. UPS valence-band spectra } & 36\end{array}$

$\begin{array}{ll}\text { 5.2.4. XAS measurements } & 37\end{array}$

5.3. Growth and structure of ultrathin $\mathrm{FeO}(111)$ films $\quad 40$

5.3.1. FeO(111) layer-by-layer growth 40

5.3.2. Atomic resolution STM and SPA-LEED measurements 41

5.3.3. Models of coincidence structures $\quad 45$

5.3.4. Defect structures $\quad 49$

5.3.5. Discussion $\quad 50$

5.4. Growth of $\mathrm{Fe}_{3} \mathrm{O}_{4}(111)$ and $\alpha-\mathrm{Fe}_{2} \mathrm{O}_{3}(0001)$ multilayer films 53

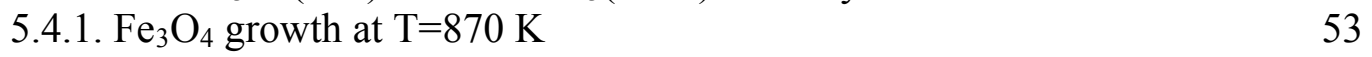

5.4.2. $\mathrm{Fe}_{3} \mathrm{O}_{4}$ growth at $\mathrm{T}=1000 \mathrm{~K} \quad 55$

5.4.3. Morphology of $\mathrm{Fe}_{3} \mathrm{O}_{4}(111)$ and $\alpha-\mathrm{Fe}_{2} \mathrm{O}_{3}(0001)$ multilayer films 56

5.4.4. $\mathrm{Fe}_{3} \mathrm{O}_{4}-\mathrm{Pt}$ interface structure $\quad 57$

5.4.5. Discussion $\quad 58$

5.5 Surface structures on $\mathrm{Fe}_{3} \mathrm{O}_{4}(111)$ films 62

5.5.1. Structures formed below $\mathrm{T}=1000 \mathrm{~K} \quad 62$

5.5.2. Structure formed at $\mathrm{T}=1000 \mathrm{~K} \quad 62$

5.5.3. Discussion $\quad 69$

5.6. Surface structures on $\alpha-\mathrm{Fe}_{2} \mathrm{O}_{3}(0001)$ films

- 2 -"2kyEi9FYSQjBVrZxIPQ.FHIAC_WRa02_review.doc", Datum: 19.02.03 
5.6.1. Pressure dependence of surface structures $\quad 71$

$\begin{array}{ll}\text { 5.6.2. LEED analysis } & 73\end{array}$

$\begin{array}{ll}\text { 5.6.3. Calculations } & 77\end{array}$

5.6.4. Discussion $\quad 78$

$\begin{array}{lr}\text { 5.7. Summary } & 80\end{array}$

6. Adsorption studies $\quad \mathbf{8 1}$

$\begin{array}{lr}\text { 6.1. Ethylbenzene adsorption } & 81\end{array}$

$\begin{array}{ll}\text { 6.1.1. Thermal desorption spectroscopy } & 81\end{array}$

6.1.2. UPS measurements - adsorbate identification and thermodynamics $\quad 85$

6.1.3. Electronic structure of adsorbates $\quad 91$

6.1.4. NEXAFS measurements 94

6.1.5. Discussion $\quad 96$

6.2. Water adsorption 98

6.2.1. Thermal desorption spectroscopy 98

6.2.2. UPS measurements - adsorbate identification and thermodynamics 99

6.2.3. Discussion 106

$\begin{array}{lr}\text { 6.3. Styrene adsorption } & 109\end{array}$

$\begin{array}{lr}\text { 6.4. Summary } & 110\end{array}$

$\begin{array}{lr}\text { 7. Reactivity studies } & 112\end{array}$

$\begin{array}{ll}\text { 7.1. Reaction studies at low pressures } & 113\end{array}$

$\begin{array}{ll}\text { 7.2. Reaction studies at elevated pressures } & 114\end{array}$

$\begin{array}{ll}\text { 7.2.1. Batch-reactor experiments } & 114\end{array}$

$\begin{array}{ll}\text { 7.2.2. Post-reaction analysis } & 116\end{array}$

$\begin{array}{ll}\text { 7.3. Discussion } & 117\end{array}$

$\begin{array}{lr}\text { 8. Conclusion } & 119\end{array}$

$\begin{array}{ll}\text { Acknowledgement } & 121\end{array}$

$\begin{array}{ll}\text { References } & 121\end{array}$ 


\section{Acronyms}

A-D

AES

AF

APB

BE

CCT

CMA

EB

fcc

FWHM

hep

HRTEM

ISS

LEED

ML

MBE

MO

NEXAFS

PEEM

QMS

SATLEED

SIMS

SPA-LEED

St

STM

TDS

TEM

TPR

UHV

UPS

XAS

XPD

XPS

XRD
Adsorption-Desorption

Auger-Electron Spectroscopy

Attenuation Factor

Antiphase Boundary

Binding Energy

Constant Current Topograph

Cylindrical Mirror Analyser

Ethylbenzene

face centered cubic

Full-Width at Half-Maximum

hexagonal close packed

High-Resolution Transmission Electron Microscopy

Ion Scattering Spectroscopy

Low-Energy Electron Diffraction

Monolayer

Molecular Beam Epitaxy

Molecular Orbital

Near-Edge X-ray Absorption Fine Structure Photoelectron Emission Microscopy/Microscope

Quadrupole Mass Spectroscopy/Spectrometer

Symmetrized Automated Tensor LEED

Secondary Ion Mass Spectrometry

Spot-Profile Analysis LEED

Styrene

Scanning Tunneling Microscopy/Microscope

Thermal Desorption Spectroscopy

Transmission electron microscopy

Temperature Programmed Reaction

Ultra-High Vacuum

Ultraviolet Photoelectron Spectroscopy

$\mathbf{X}$-ray Absorption Spectroscopy

$\mathbf{X}$-ray Photoelectron Diffraction

X-ray Photoelectron Spectroscopy

$\mathbf{X}$-ray Diffraction 


\section{Introduction}

Metal-oxides constitute an important class of materials that are involved in environmental science, electrochemistry, biology, chemical sensors, magnetism and other fields. One of their most important applications is heterogeneous catalysis. Metal-oxides are used for synthesizing many organic compounds via selective oxidation, dehydrogenation, isomerization and other chemical processes [1]. Not much is known about atomic scale mechanisms of catalytic reactions on metal-oxides, and our understanding of basic metal-oxide surface chemistry greatly lags behind that of semiconductors and metals. This basic understanding, in particular the relationships between the catalytic function of oxide materials and their crystallographic structures and chemical compositions in the surface region, is needed for a targeted catalyst development. On pure metal catalysts the delocalized electrons can easily be transferred resulting in redox chemistry reaction mechanisms. Oxides are ionic compounds exposing acidic and basic sites, which may control their surface chemical properties. At the same time, the redox chemistry looses in importance, because the electronic states are separated by the bandgap and become less accessible. Metallic and semiconducting oxides with small bandgaps allow a combination of redox and acid-base chemistry, whereas highly ionic oxides with large bandgaps become pure acid-base catalysts. In this context, a large amount of work has been performed over polycrystalline oxide catalyst samples, resulting, for example, in the important concept of surface coordinatively unsaturated sites $[2,3]$. But since electronic surface structures and acid-base properties of oxides may vary greatly with the surface orientation, the conclusions to be drawn from studies on polycrystalline samples are limited. Therefore, a direct elucidation of atomic structural elements involved in chemisorption processes and catalytic conversions requires samples exposing one defined surface orientation. This has led to an increasing number of surface science studies on single-crystal metal-oxide samples within the last decade, accompanied by theoretical work, as summarized in several recent books [4-6].

Technical oxide catalysts are polycrystalline materials that contain several components and promotor additives with precise proportions that have been optimized in order to give the optimal activities and selectivities. Compared to metals, more complex reaction mechanisms may exist. For example, lattice $\mathrm{O}$ atoms may be removed from the catalyst surface and replaced at the end of a catalytic cycle (Mars-van-Krevelen mechanism [7]). In the surface science approach, the elementary steps constituting the reaction mechanism are studied under ultrahigh vacuum (UHV) conditions. By varying the model system surface structures, i.e., by choosing different surface orientations or by varying the oxide composition, structure-reactivity correlations can be studied systematically. The most active phase in the case of structure-sensitive reactions and the atomic structural elements involved can be determined this way. In a second step, the problem of the "pressure-material gap" has to be addressed: Does the mechanism change when going to realistic operation conditions at high gas pressures and temperatures? This may happen for several reasons, as outlined in Section 2.6. One can approach this question by combining surface science experiments with kinetic reaction studies at high pressures [8]. In a final step, the model system activities may be compared to those of real polycrystalline catalyst samples. Several reactions peformed over metal catalysts were analyzed sucessfully by this integrated surface science approach, the most prominent one being the ammonia synthesis [9].

The structural complexity of oxide catalysts constitutes the main problem when tackling catalysis research in an integrated surface science approach. The preparation of metal-oxide surfaces with defined structures and stoichiometries turned out to be very difficult $[10,11]$. Single-crystal samples are sometimes not available and often contain impurities that segregate to the surface in an uncontroled way. Often oxides are electrical insulators that restrict or prevent electron spectroscopy or scanning tunneling microscopy (STM) measurements. Other experimental problems are related to sample mounting, heating problems due to the poor thermal conductivities of oxide materials and temperature control by thermocouples. In particular, the performance of high pressure reaction experiments over

- 5 -"2kyEi9FYSQjBVrZxIPQ.FHIAC_WRa02_review.doc", Datum: 19.02.03 
single-crystal samples seems problematic and has not been done so far, since it is often difficult to clean and restore these samples after reaction experiments, when they are covered with carbonaceous deposits or other side products. Most of these problems can be avoided by using thin epitaxial oxide films grown onto conducting substrates. Often their conductivity is sufficient, their bulk contamination can be controlled and is limited anyway by the small film thickness and, if contaminated, the films can be removed from the substrate and freshly prepared. As outlined in Section 2.7, much progress has been made in preparing ordered oxide films within the last 10 years.

Here we review the progress of the first comprehensive approach to tackle a metal-oxide catalyzed reaction by an integrated surface science study. As a particular reaction the dehydrogenation of ethylbenzene to styrene was chosen, which is performed industrially over Fe-oxide based catalysts in the presence of steam [12]. For studying structure-reactivity relationships in a systematic way, $\mathrm{FeO}(111), \mathrm{Fe}_{3} \mathrm{O}_{4}(111)$ and $\alpha-\mathrm{Fe}_{2} \mathrm{O}_{3}(0001)$ films were grown heteroepitaxially onto $\mathrm{Pt}(111)$ substrates. The atomic surface structure and surface defects of these films was characterized by STM and LEED (Low-energy electron diffraction) crystallography, supplemented by high-resolution transmission electron microscopy (HRTEM) investigations. Their chemical composition and electronic structure were studied by Auger electron spectroscopy (AES) and photoelectron spectroscopy (UPS, XPS). The interaction of gases involved in the styrene synthesis reaction (water, ethylbenzene and styrene) with these model catalyst films was studied by thermal desorption spectroscopy (TDS), XPS, UPS and near edge X-ray absorption fine structure (NEXAFS) measurements. In order to bridge the pressure-gap and obtain information more relevant for real catalysts, the UHV experiments were combined with catalytic reaction experiments at high gas pressures using a batch reactor system, followed by postreaction analysis with AES and photoemission electron microscopy (PEEM).

In real catalysis, potassium promoted Fe-oxides are used. Although experiments with potassium promoted model catalyst films were started, we do not include them into this review. The addition of potassium increases the complexity of the system considerably and the surface characterization of ternary $\mathrm{K}-\mathrm{Fe}-\mathrm{O}$ films is only at its beginning.

The review is organized as follows: The basic concept of the surface science approach and the experimental methods applied in this work are outlined in Section 2. In Section 3 the experimental equipment is described, followed by an introduction to the styrene-synthesis reaction in Section 4 and the characterization of the epitaxial Fe-oxide films with respect to their crystallographic structures and chemical compositions in Section 5. The adsorption studies are presented in Section 6 and the high pressure reaction studies in Section 7. The conclusions and outlook are given in Section 8.

\section{Basic concepts}

\subsection{Adsorption process}

The first step of every surface reaction is the adsorption of the reactants from the gas-phase onto the surface. Langmuir first introduced the concept of the existence of specific adsorption sites given by the surface lattice $[13,14]$. Molecules in the gas-phase striking the surface might bounce back into the gasphase or get adsorbed onto one of these sites. If the interaction between the adsorbed molecules and the surface leads to a chemical bond the process is called chemisorption, if no chemical bond is established the process is classified as physisorption [15]. After adsorption, the free energy of the system consisting of the clean surface and the gas-phase molecules is lowered, where the energy lowering is given by the isosteric heat of adsorption $\mathrm{q}_{\mathrm{st}}$. The surface phenomenom of adsorption can be treated macroscopically by chemical thermodynamics, and the isosteric heat of adsorption can be derived this way, without using atomic concepts [15-20]. As outlined in the next section, the isosteric heat of adsorption can be measured by calorimetry or by a Clausius-Clapeyron analysis of isostere

$$
\text { - } 6 \text {-"2kyEi9FYSQjBVrZxIPQ.FHIAC_WRa02_review.doc", Datum: 19.02.03 }
$$


data, where the latter method is rigorously only correct for adsorbents weakly interacting with the surface. One speaks of chemisorption, if the energy deliberated in bond formation is higher than about $40 \mathrm{~kJ} / \mathrm{mol}$. However, chemisorption requires a direct interaction between substrate and adsorbate charge densities, and one has to look at the electronic structure of the system to really differentiate between physisorption and chemisorption, for example, by spectroscopic techniques. The connection between macroscopic thermodynamics and the microscopic view of adsorbate energetics is given by the potential energy diagrams shown in Fig. 2.1 as quasi-one-dimensional Lennard-Jones representations. This approach, first introduced by Lennard-Jones [21], describes the interaction between the surface and a gas-phase molecule approaching the surface as a superposition of a longrange attractive force and a short-range repulsive force. Today these interactions can be described on the basis of ab-initio quantal calculations using various approaches [22, 23]. Not considering the question of how the gas-phase molecule gets trapped into the adsorbed state, Fig. 2.1a illustrates associative molecular adsorption $\left(\mathrm{E}_{\mathrm{ads}}\right)$, where the gas-phase molecule only sees a downhill potential, until it reaches the equilibrium position $\mathrm{z}_{0}$. Figure $2.1 \mathrm{~b}$ illustrates molecular adsorption $\left({ }^{2} \mathrm{E}_{\mathrm{ads}}\right)$ via a weakly bound physisorbed precursor state $\left({ }^{1} \mathrm{E}_{\mathrm{ads}}\right)$. Figure $2.1 \mathrm{c}$ illustrates a dissociative chemisorption $\left({ }^{\mathrm{d}} \mathrm{E}_{\mathrm{ads}}\right)$ via a molecular precursor state $\left({ }^{\mathrm{m}} \mathrm{E}_{\mathrm{ads}}\right)$, where the activation energy $\mathrm{E}_{\text {ads }}^{*}$ has to be overcome in order to get from the molecular precursor into the chemisorbed state. With the above-mentionen restriction in mind, the adsorption energies $E_{\text {ads }}$ in Fig. 2.1 correspond to the isosteric heats of adsorption $\mathrm{q}_{\mathrm{st}}$, if referred, for example, to one mole of adsorbent.
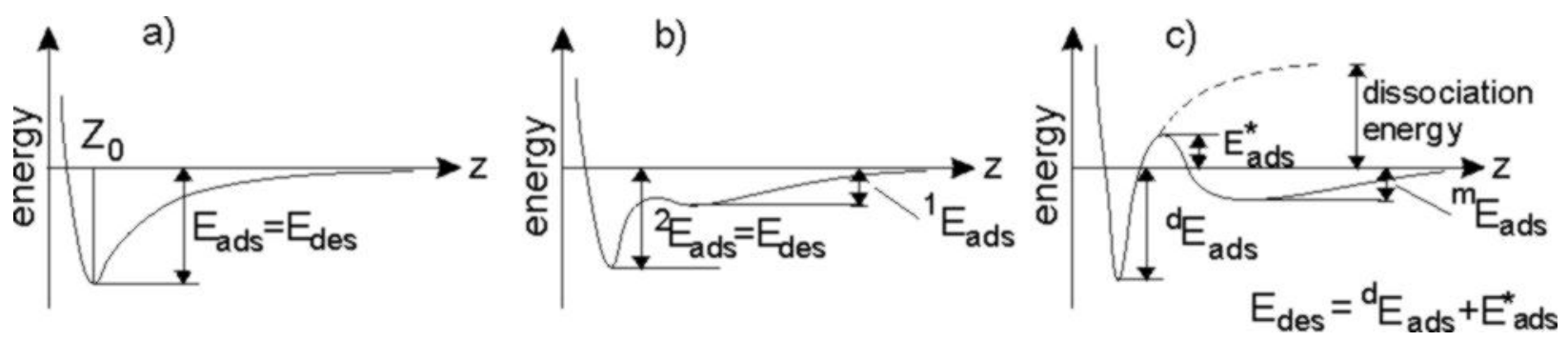

Fig. 2.1. Potential energy diagrams in one-dimensional Lennard-Jones representation. (a) associative chemisorption $\left(E_{a d s}\right)$; (b) associative chemisorption $\left({ }^{2} E_{a d s}\right)$ with precursor $\left({ }^{1} E_{a d s}\right)$; (c) dissociative chemisorption $\left({ }^{d} E_{a d s}\right)$ with molecular precursor $\left({ }^{m} E_{a d s}\right)$ and activation barrier $\left(E^{*}{ }_{a d s}\right)$. Desorption energies $E_{d e s}$ are also indicated.

The strength of the chemisorption bond and the existence of weakly bound precursor states is determined by the atomic-orbital interactions between the substrate surface and the adsorbate molecule. An ultimate goal in catalysis research is to understand the correlations between the geometric and electronic structure of the surface, the geometric shape and electronic structure of the involved molecules and the observed macroscopic catalytic reactivity. One distinguishes between structure-sensitive and structure-insensitive reactions. In the first case, the adsorption depends on the geometric surface.

On metals, many structure-sensitive reactions are found. The dissociative adsorption of $\mathrm{N}_{2}$ on Fe surfaces, which is the rate determining step of the ammonia synthesis reaction, turns out to be highly structure-sensitive [24-26]. Other examples are $\mathrm{CO}$ adsorption [27], $\mathrm{H}$ chemisorption [28] or $\mathrm{O}$ chemisorption [29, 30]. The substrate-adsorbate interaction can be described by interactions between the molecular orbitals of the adsorbate and the delocalized bands of the metal substrate. CO chemisorption on transition metal surfaces, for example, is well-explained by the Blyholder model [31]: The $\mathrm{C}$ lone-pair orbital of $\mathrm{CO}$ donates electronic charge into the highly dispersed empty conduction bands of the metal substrate, which are formed by hybridisation of $\mathrm{d}$ and s states, thereby establishing a metal-adsorbate $\sigma$-donor bond. At the same time, electronic charge is backdonated from 
the occupied metal d-band into the empty $2 \pi^{*}$ molecular orbital of $\mathrm{CO}$, forming a metal-adsorbate $\pi$ acceptor bond, which results in strong bonding with chemisorption energies between 100 and 200 $\mathrm{kJ} / \mathrm{mol}$. The charge transfer between substrate and adsorbate can be viewed as a donor-acceptor process, which determines the adsorbate geometry and which also can be described by ab-initio calculations using density-functional theory [32]. The backdonation from the metal into antibonding molecular-orbital states weakens the bonding within the adsorbate molecule, which can lead to dissociation of the adsorbate molecule [33]. A correlation between the d-band center of transition metal and alloy surfaces and the chemisorption energy of $\mathrm{CO}$ and other small molecules has been established $[32,34]$. Enhanced $\mathrm{CO}$ adsorption energies at surface defect sites of bimetallic surfaces, such as atomic step edges or kinks, has been found recently, which was attributed to the reduced coordination of these metal sites [35-37].

A more complex adsorption behavior is encountered on metal-oxide surfaces. The most detailed understanding has evolved from electronic structure calculations for small probe molecules adsorbed on metal-oxide surfaces, such as $\mathrm{NiO}, \mathrm{MgO}$, and $\mathrm{ZnO}$. These calculations revealed a very different interaction, when compared to the donor-acceptor interaction discussed above [38-47]. The bonding of $\mathrm{CO}$ and other molecules on these surfaces cannot be described by the Blyholder model, as only very moderate $\sigma$-donation or $\pi$-backdonation occurs. Instead, electrostatic forces and Pauli repulsion dominate. For example, on the strongly ionic $\mathrm{NiO}(100)$ surface, the interaction between the multipolar moment of the molecular electron density and that of the ionic surface determines the adsorption energy. One reason is the different band structure of metal-oxides, where the metal s-band usually is strongly destabilized and located higher in energy, so that only a weak substrate-adsorbate orbital overlap becomes possible and no strong $\sigma$-donor bond can be established. On the other hand, no $\pi$ acceptor backbond is possible for steric reasons, because the $\mathrm{CO}$ molecule cannot approach the Ni ions close enough, since they get repelled by the large closed shell O ions on the (100) surface [10]. This Pauli repulsion balances the electrostatic attractive forces, resulting in rather weak adsorption energies ranging from $10-50 \mathrm{~kJ} / \mathrm{mol}$ for $\mathrm{CO}$ on typical oxide surfaces, which correspond to physisorption or weak chemisorption [48-50]. Nevertheless, the adsorbed molecules adopt favored orientations on the surface [51], but, because of the weak interaction, different orientations become possible, as observed for $\mathrm{CO}$ on $\mathrm{Cr}_{2} \mathrm{O}_{3}(0001)$ [52]. Vibration spectroscopy data also are in line with this picture of adsorbed molecules on oxide surfaces $[48,53,54]$.

From a more chemical point of view, the adsorption on metal-oxides can also be discussed in terms of acid-base interactions [3,5]. This concept focuses on the localized electrostatic interaction between substrate atoms and adsorbate molecules, using the concept of coordinative unsaturation. Whereas in bulk oxides all metal cations are coordinatively saturated by the surrounding $\mathrm{O}$ anions, coordinatively unsaturated metal and $\mathrm{O}$ sites are exposed at the surface. Since metal-oxides are ionic compounds, cation sites are Lewis acids, which can interact with donor molecules that expose a lone-pair electron, such as $\mathrm{H}_{2} \mathrm{O}, \mathrm{NH}_{3}$ or $\mathrm{CO}$, through a combination of electrostatics (ion-dipole attraction) and orbital overlap. In this way, a Lewis site $\mathrm{M}^{\mathrm{n}+}$ may act as an acceptor for free-electron pairs of a molecularly adsorbed basic species B:

$$
\mathrm{M}^{\mathrm{n}+}+: \mathrm{B}(\text { gas }) \leftrightarrow \mathrm{M}^{\mathrm{n}+}: \mathrm{B} .
$$

The strength of the acid sites depends on the charge and size of the cations [55] and can be measured by spectroscopic investigation of adsorbed probe molecules [56, 57]. An essential feature of acid-base reactions is that no complete charge transfer takes place. Adsorbate molecules may be classified as electron donors or acceptors, according to the direction of charge transfer, but the electrons are not free and no complete release or capture of electrons occurs. $\mathrm{O}$ anion sites are Brønsted bases that can accept protons, for example, deprotonate a $\mathrm{C}-\mathrm{H}$ group of a hydrocarbon molecule, thereby forming an alkyl and a $\mathrm{OH}$ group on the surface. Surface $\mathrm{OH}$ groups can act as Brønsted acid sites, which dissociate to protonate adsorbed bases ( $*$ denotes a surface lattice atom): 


$$
\mathrm{B}+\mathrm{HO}-* \leftrightarrow \mathrm{BH}^{+}+\mathrm{O}^{-}-*
$$

Oxide surfaces usually become fully covered with adsorbed water and $\mathrm{OH}$ groups once they become exposed to the moisture in the atmosphere. Molecular adsorption of water can occur at metal Lewis sites, mostly with the $\mathrm{O}$ atom oriented towards the surface [58]. Heterolytic dissociative adsorption of water occurs by the simultaneous action of a Lewis acid site and a Brønsted base site, where a $\mathrm{OH}$ group is bound to the metal cation and $\mathrm{H}^{+}$is transferred to a neighboring basic $\mathrm{O}$ anion. Equ. (2.3) illustrates both cases:

$$
\begin{gathered}
\mathrm{M}^{\mathrm{n}+}+\mathrm{H}_{2} \mathrm{O} \leftrightarrow \mathrm{M}^{\mathrm{n}+} \mathrm{OH}_{2}, \\
\mathrm{H}_{2} \mathrm{O}+\mathrm{M}^{\mathrm{n}+}+\mathrm{M}^{\mathrm{n+}} \mathrm{O}^{2-} \leftrightarrow 2 \mathrm{HO}^{-}-\mathrm{M}^{\mathrm{n}+} .
\end{gathered}
$$

This hydroxylation was observed on single-crystalline surfaces of several transition metal-oxides [59, 60-63]. Polar surface planes can be stabilized by hydroxylation, because it reduces excess surface charge and, therefore, the electrostatic surface dipole moment (see Section 2.8). Upon dehydroxylation, these surface planes reconstruct, in order to establish a more stable configuration [64$66]$.

In many selective oxidation and dehydrogenation reactions, $\mathrm{O}$ atoms are transferred into adsorbed organic molecules [3]. In some reactions, lattice $\mathrm{O}$ atoms are involved, which can be added to adsorbed molecules, not as an $\mathrm{O}^{2-}$ anion, but as a neutral $\mathrm{O}$ atom. This is accompanied by a reduction of the oxide surface, such as

$$
\mathrm{CO}+\mathrm{O}_{\text {latt }}^{2-} \rightarrow \mathrm{CO}_{2}+2 \mathrm{e}^{-}
$$

The removed lattice $\mathrm{O}$ atoms can be replaced by adsorbed $\mathrm{O}$ species that migrate to the $\mathrm{O}$ vacancy site at the surface, thereby reoxidizing the catalyst surface, which is known as the Mars van Krevelen mechanism [67].

In summary, adsorption on oxide surfaces is determined both by localized electrostatic interactions and by electronic donor-acceptor interactions between molecular orbitals of the adsorbate and delocalized valence and conduction bands of the substrate. The electrostatic interaction clearly dominates for strongly ionic wide band-gap insulator oxides, because no strong donor-acceptor interaction is possible between the energetically high lying empty conduction bands and the much lower lying occupied molecular orbitals of the adsorbates. For oxides with smaller bandgaps, an orbital overlap becomes more favorable, and the dominance of the electrostatic interaction is reduced. In both cases, the geometric surface structure and the surface termination will determine the steric situation, which defines how close the adsorbate molecule can approach the cation and anion sites on the surface and, therefore, the possible degree of orbital overlap. Here the structure sensitivity for adsorption on metal-oxide surfaces comes into play. It is obvious that the termination of polar metal-oxide surface planes (i.e., which species are exposed at the outermost surface plane), as well as surface relaxations or reconstructions play an important role. In the case of $\mathrm{CO}$ adsorption on the unpolar $\mathrm{NiO}(100)$ surface discussed above, the $\mathrm{CO}$ molecule cannot interact with the metal cations, because it is sterically hindered by the large $\mathrm{O}$ anions to approach these sites. A metal-oxide surface with metal cations relaxed outward from the surface $O$ plane is expected to be more reactive for that reason, whereas a surface terminated by a close-packed $\mathrm{O}$ layer will be completely inert with respect to the adsorption of basic species.

The concept of coordinative unsaturation starts from the fact that a surface site has a lower coordination than the corresponding bulk site. At atomic surface defects, such as steps, kinks, adatoms or vacancies the coordination becomes even lower. Metal cations and $\mathrm{O}$ anions, located at such surface defect sites, will have increased the acidic and basic properties, respectively. Such species can be more easily removed from the solid lattice, which comes into play in oxidation reactions involving lattice $O$. This explains the stability and mostly lower reactivity of defect free, regular oxide surfaces, and the 
higher reactivity of more defective oxide surfaces. However, there is not much direct experimental evidence for the higher reactivity of surface defect sites of metal-oxides. Studies on single-crystalline $\mathrm{NiO}(100)$ and (111) surfaces revealed water to dissociatively adsorb at atomic defect sites [64, 68], and it is well-known that completely hydroxylized oxides are not active, unless they get dehydroxylated, so that highly unsaturated sites are exposed again [2].

\subsection{Thermodynamics and energetics of adsorption}

Even without using atomic concepts, the surface phenomenom of adsorption can be treated by chemical thermodynamics, which derive the relationship between quantities, such as gas pressure, temperature, volume, surface area and others [15, 17-19]. Formulating the Gibbs fundamental equations for the full system consisting of adsorbent (surface) and adsorbate (including the gas-phase), the thermodynamic parameters of adsorbate and adsorbent can be separated, provided they represent truly separable subsystems. Therefore, this derivation is strictly applicable only for weakly interacting physisorbate systems and not for chemisorbate systems. With this restriction in mind, and assuming equilibrium between the gas-phase and the adsorbate phase, a Clausius-Clapeyron analysis yields for a constant adsorbate surface coverage $\Theta[20]$ :

$$
\left(\frac{\partial \ln p}{\partial T}\right)_{\theta}=\frac{-q_{s t}}{R T^{2}} .
$$

Rewriting and integrating this equation over reasonably small pressure and temperature intervals yields

$$
\ln \left(\frac{p_{1}}{p_{2}}\right)=\frac{q_{s t}}{R}\left(\frac{1}{T_{1}}-\frac{1}{T_{2}}\right),
$$

for two pairs of temperature $\mathrm{T}$ and gas pressure $\mathrm{p}$, which produce the same adsorbate coverage. Experimentally, different surface coverages can be established and determined by several techniques [16]. Therefore, the isosteric heat of adsorption can be determined as a function of the surface coverage by measuring adsorption isotherms or isobars, if true equilibrium is established between the adsorbate and the gas-phase. For each constant surface coverage, a semilogarithmic plot of $\ln p$ versus 1/T should yield a straight line with the slope being the isosteric heat of adsorption, as shown for adsorption of ethylbenzene on $\mathrm{Pt}(111)$ in Fig. 2.2 [69]. This analysis was also performed for other adsorption systems, such as $\mathrm{Xe} / \mathrm{Ni}(100)$ [70]. However, a reliable determination of the surface coverage, under adsorption-desorption equilibrium conditions, is not straightforward and has to be carried out carefully in order to avoid mistakes. Also, the establishment of adsorption-desorption equilibrium usually does not strictly represent thermodynamic equilibrium, because in typical UHV experiments the temperature of the gas-phase differs from the sample temperature. As shown in Section 2.4, this causes no problem, if the adsorption kinetics are determined by the sample temperature.

Nevertheless, the most direct method for the determination of heats of adsorption would be calorimetric measurements, which is difficult, because of the very small temperature rise caused by the adsorption of monolayer or submonolayer quantities. King and co-workers succeeded in performing such measurements on thin single-crystal samples by applying molecular-beam techniques in combination with infrared radiation emission measurements $[72,73]$. 

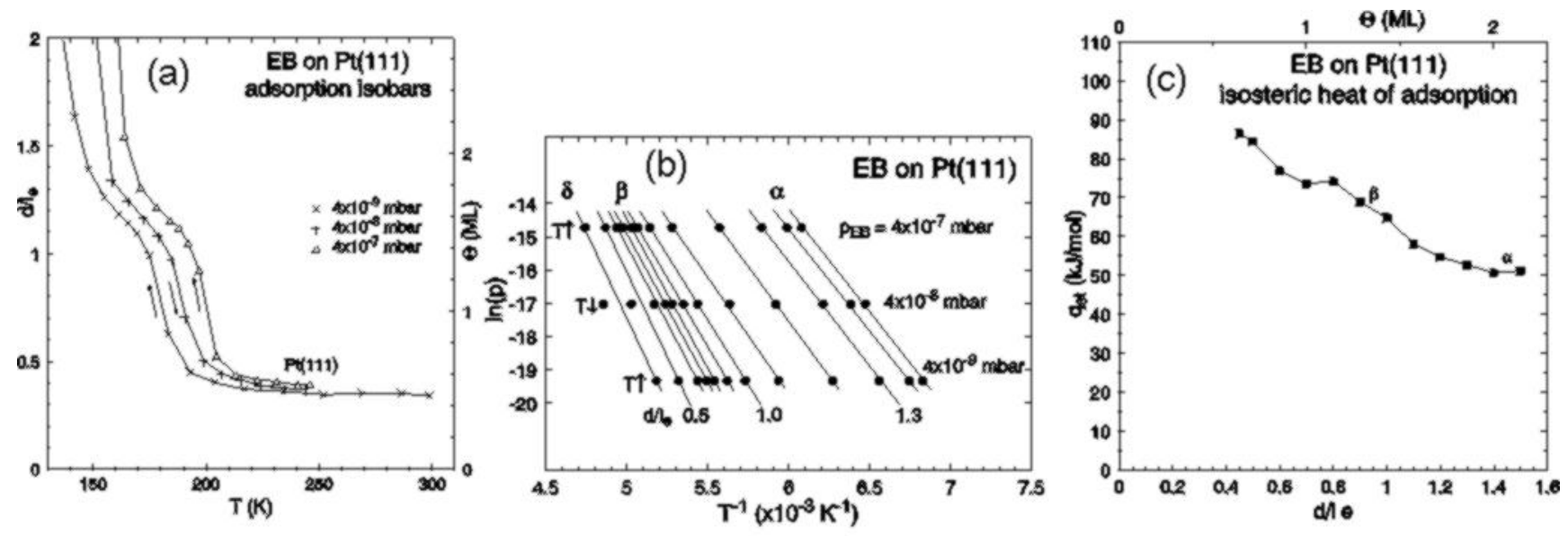

Fig. 2.2. Determination of isosteric heat of adsorption from experimental adsorption isobars for adsorption of ethylbenzene (EB) on Pt(111) [71].

An easier and frequently used method for the determination of the activation energy for desorption $\mathrm{E}_{\mathrm{des}}$ (which in the case of non-activated adsorption is equal to the adsorption energy $\mathrm{E}_{\text {ads }}$ or the isosteric heat of adsorption $\mathrm{q}_{\mathrm{st}}(\Theta)$ see Fig. 2.1) is thermal desorption spectroscopy (TDS, often also termed temperature programmed desorption, TPD) [16, 74-78]. The surface is held at a temperature $T_{0}$ and is exposed to a defined amount of gas, often given in Langmuir units $\left(1 \mathrm{~L}=1.33^{-6} \mathrm{mbar} \times \mathrm{sec}\right)$. It is then heated in vacuum upto a temperature where all the molecules have desorbed. Linear temperature ramps with heating rates $\beta$ ranging from $1-10 \mathrm{~K} / \mathrm{sec}$ are typically applied, so that the sample temperature is given by $T=T_{0}+\beta t$. The concentration of desorbing species is measured with a quadrupole mass spectrometer (QMS) placed right in front of the sample, which yields a desorption trace for the adsorbed molecular species. The total area under the desorption trace corresponds to the total amount of adsorbed species. If the pumping speed is high enough, so that no readsorption takes place during the experiment, the mass spectrometer signal is proportional to the desorption rate $r_{\text {des }}$ as given by the Polanyi-Wigner equation [79]

$$
r_{d e s}=-\frac{\sigma_{A} d \Theta}{d t}=-v_{n}(\Theta) \sigma_{A}^{n} \exp \left(-\frac{E_{d e s}(\Theta)}{k T}\right) \Theta^{n} \text {. }
$$

$\sigma_{A}$ is the density of adsorption sites, $\Theta$ the relative surface coverage with the saturation coverage corresponding to $\Theta=1, n$ denotes the order of desorption, $E_{d e s}(\Theta)$ is the activation energy of desorption and $v(\Theta)$ the preexponetial frequency factor. This equation is the basis for the analysis of TDS, which can be performed in different ways. Both the activation energy of desorption and the preexponetial frequency factor can depend on the surface coverage, because of adsobate-adsorbate interactions.

Since two unknown and coverage dependent parameters are contained in the Polanyi-Wigner equation, $E_{\text {des }}(\Theta)$ and $v(\Theta)$, their determination is not straightforward. Figure 2.3 demonstrates the exactest analysis needing no assumptions for one of these parametes. It is based on a set of desorption traces (a) with different initial coverages $\Theta_{0}$ and was first propose as "complete analysis" by King [76]. For every desorption trace, the coverage-temperature curve is derived by integration from the temperature $T_{0}$ upto a temperature where all molecules have desorbed (b). This yields coveragetemperature curves starting at $\Theta=\Theta_{0}$ for $T=T_{0}$ and decreasing with increasing temperature until $\Theta=0$. Each intersect of a straight line representing a constant surface coverage $\Theta$ (dashed line in Fig. 2.3b) with the coverage-temperature curves gives a temperature $T$, for which the desorption rate can be 
obtained from the corresponding desorption trace in Fig. 2.3a. This yields pairs of $r_{d e s}-T$ values that form straight lines in an Arrhenius plot $\ln \left(r_{d e s}\right)$ versus $1 / T$ as shown in Fig. $2.3 \mathrm{c}$, where $E_{d e s}$ can be obtained from the slope and $v(\Theta)$ from the intersect.

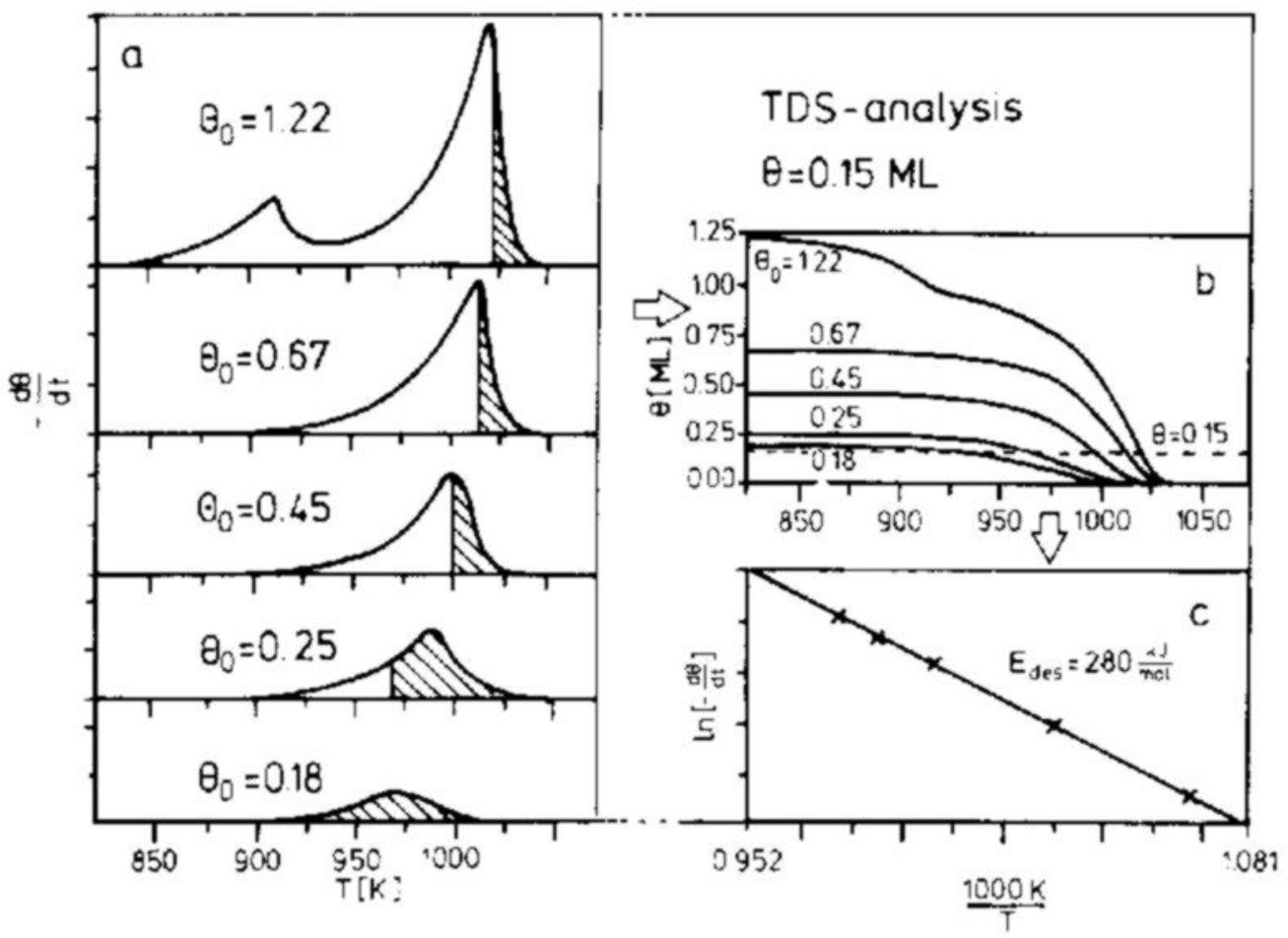

Fig. 2.3. Determination of desorption energies via complete analysis of TDS data. (a) TDS desorption traces measured with increasing initial coverages $\Theta_{0}$; (b) $\Theta-T$ curves obtained by integration of desorption traces. Analysis is performed for $\Theta=0.15$; (c) Arrhenius plot $\ln (\mathrm{d} \Theta / \mathrm{d} t)=\ln \left(r_{\text {des }}\right)$ versus $1 / T$ yields desorption energy according to (2.7). Data are for $\mathrm{Ag} / \mathrm{Ru}(0001)$ [80].

An alternative way is to compute TDS traces from the Polanyi-Wigner equation assuming that $E_{d e s}$ and $v$ are coverage independent. From the best-fit curve, the desorption energy and frequency factor is obtained [81]. However, this procedure is not self-consistent, because $E_{\text {des }}$ and $v$ are not, in general, coverage independent. The simple so-called Redhead analysis is based on the peak maximum temperatute $T_{\max }$ observed in the TDS spectrum and can easily be derived from the Polanyi-Wigner equation [75]. It requires the assumption of a reasonable value for the frequency factor $v$ and is only valid for first-order desorption kinetics

$$
E_{\text {des }}=R T_{\max }\left[\ln \left(v T_{\max } / \beta\right)-3.46\right] .
$$

A final method is the threshold or leading edge analysis [82-84], where only the first part of the TDS trace is considered, until a few percent of the adsorbate have desorbed. The coverage $\Theta$ on the right-hand side of the Polanyi-Wigner equation is set to the initial coverage value $\Theta_{0}$ of the corresponding TDS trace, and a plot of the logarithm of the desorption rate $r_{\text {des }}$ versus $1 / T$ from the initial temperature $T_{0}$ up to the temperature $T$, where some percent have desorbed, yields a straight line from which $E_{d e s}\left(\Theta_{0}\right)$ and $v\left(\Theta_{0}\right)$ can be determined. In this way, $E_{d e s}$ and $v$ can be obtained as a function of coverage by analyzing TDS traces with different initial coverages $\Theta_{0}$. Other analysis procedures are given in the literature $[80,85,86]$. 


\subsection{Langmuir isotherm}

Heterogeneous catalytic reactions take place at pressures and temperatures much higher than those applied in TDS and other surface-science experiments. Therefore, it is desirable to be able to extrapolate the surface coverage from low-pressure and low-temperature measurements to high pressures and temperatures using isotherms or isobars. The rate of adsorption is proportional to the flux $F$ of molecules striking the surface per unit time and unit area. It can be calculated using kinetic gas theory to be $F=p / \sqrt{2 \pi m k T}$ [87] with $p$ being the gas pressure, $m$ the molecule mass and $k$ the Boltzmann constant. The adsorption rate depends further on the probability of the incident particles to stick to the surface, the sticking coefficient $s$, which depends on the surface and the adsorbate molecules under investigation, can assume values between 0 and unity. The sticking coefficient for zero coverage is called initial sticking coefficient $s_{0}$.

Thermodynamic adsorption-desorption equilibrium means that the rates of adsorption and desorption are equal:

$$
r_{a d s}+r_{d e s}=0 \text {. }
$$

The desorption rate is given by (2.7). The adsorption rate is given by the product of the incident flux $F$, the initial sticking probability $s_{0}$, which here is expressed as the product of $s^{*}$ (initial sticking probability for non-activated adsorption) and a Boltzmann term with the activation energy for adsorption $E^{*}{ }_{a d s}$. The coverage dependence of the sticking probability is expressed by the function $f(\Theta)$ :

$$
r_{a d s}(\Theta)=\frac{\sigma_{A} d \Theta}{d t}=\frac{p}{\sqrt{2 \pi m k T}} s^{*} \exp \left(-\frac{E^{*}{ }_{a d s}}{k T}\right) f(\Theta) .
$$

The Langmuir isotherm is based on the assumption that the sticking probability for non-dissociative adsorption is proportional to the number of unoccupied sites: $f(\Theta)=(1-\Theta)$. For dissociative adsorption, two adsorption sites are consumed per incident molecule and $f(\Theta)=(1-\Theta)^{2}$. Any adsobate-adsorbate interactions are neglected, which means that the desorption energy and preexponential factor do not depend on the surface coverage. Equating (2.7) and (2.10) and resolving for $\Theta$ yields for the desorption order $n=1$ :

$$
\Theta(p, T)=\frac{b_{1}(T) \cdot p}{1+b_{1}(T) \cdot p}, \quad b_{1}(T)=\frac{s^{*} \exp \left(q_{s t} / k T\right)}{v_{1} \sigma_{A} \sqrt{2 \pi m k T}}, \quad \mathrm{n}=1, f(\Theta)=(1-\Theta) .
$$

A similar expression can be derived for dissociative adsorption and desorption order $n=2$ [16, 88], viz.,

$$
\Theta(p, T)=\frac{\sqrt{b_{2}(T) \cdot p}}{1+\sqrt{b_{2}(T) \cdot p}}, \quad b_{2}(T)=\frac{s^{*} \exp \left(q_{s t} / k T\right)}{v_{2} \sigma_{A}^{2} \sqrt{2 \pi m k T}}, \quad \mathrm{n}=2, \quad f(\Theta)=(1-\Theta)^{2},
$$

with $q_{s t}=E_{d e s}-E_{a d s}^{*}$ is the isosteric heat of adsorption.

Despite the neglection of adsorbate-adsorbate interactions, the Langmuir isotherm describes many adsorption systems quite well. The function $f(\Theta)$ in (2.10) may also be modified to include the influence of a mobile precursor state in adsorption [88] (see Section 2.5). Other isotherms were derived where some of the assumptions for the Langmuir isotherm were released or relaxed [89] [90][91][ 92]. It should be noted that (2.11) may also be used to describe an adsorption isobar, such as those presented in Fig. 2.2, if the pressure $p$ is kept constant and the temperature $T$ contained in the terms $b(T)$ is varied. 
So far, the adsorption of single chemical species onto one type of adsorption site was considered. If several types of adsorption sites, with different adsorption energies, are present on the surface, the occupation of each site can be described separately by the Langmuir isotherms with the corresponding intrinsic kinetic parameters $\left(s_{0}, \mathrm{v}, E_{d e s}, E^{*}{ }_{a d s}\right)$. In the case of coadsorption of two different species $\mathrm{A}$ and $\mathrm{B}$ different scenarios are possible. If the species $\mathrm{A}$ and $\mathrm{B}$ prefer different types of adsorption sites without affecting each other, the simple Langmuir isotherm describes the adsorption of each species independently, and the corresponding surface coverages $\Theta_{A}$ and $\Theta_{B}$ do not depend on each other. In the case of competitive coadsorption, the simple Langmuir isotherm has to be modified by introducing the fractional coverages $\Theta_{A}$ and $\Theta_{B}$, which now depend on each other according to $\Theta_{A}+\Theta_{B}=\Theta$. For such coadsorption systems, similar expressions can be derived for their Langmuir isotherms, both for dissociative and non-dissociative adsorption, which contain the kinetic parameters of both adsorbate species [16]. The overall spatial distribution of the coadsorbed particles A and B on the surface can be very different and is determined mainly by the adsorbate-adsorbate interaction energies $\omega_{A A}, \omega_{B B}$, and $\omega_{A B}$. If attractive interactions between identical species $\mathrm{A}$ or $\mathrm{B}\left(\omega_{A A}\right.$ and $\left.\omega_{B B}\right)$ dominate over interactions between different species $\mathrm{A}$ and $\mathrm{B}\left(\omega_{A B}\right)$, the two species will not mix on the surface, but form domains with structures characteristic for the A and B phases, which is the classical case of competitive adsorption. On the other hand, if the attractive interaction $\omega_{A B}$ dominates, $\mathrm{A}$ and $\mathrm{B}$ want to become nearest neighbors and may form a composite phase with a new periodicity on the surface, which is called cooperative coadsorption. It is obvious that, for bimolecular Langmuir-Hinshelwood reactions, the reaction rate will be much higher for cooperative adsorption systems than competitive adsorption ones, which form separated domains on the catalyst surface.

\subsection{Kinetic analysis of isosteric heat of adsorption}

In Section 2.2, it was pointed out that a Clausius-Clapeyron analysis for an adsorption system revealing the isosteric heat of adsorption $q_{s t}$ is problematic, if the adsorbate and the substrate interact strongly, and if the surface temperature $T$ and the gas-phase-temperature $T_{\text {gas }}$ are different. Here we present a kinetic derivation for the determination of the isosteric heat of adsorption that circumvents these problems.

Again we start with equating the rates for adsorption (2.10) and desorption (2.7):

$$
\frac{p}{\sqrt{2 \pi m k T_{\text {gas }}}} s^{*} f(\Theta) \exp \left(-\frac{E_{\text {dds }}^{*}(\Theta)}{k T}\right)=v_{n}(\Theta) \sigma_{A} \Theta^{n} \exp \left(-\frac{E_{d e s}(\Theta)}{k T}\right) \text {. }
$$

The difference is that the temperature $T_{\text {gas }}$, which enters into the flux $F=p / \sqrt{2 \pi m k T_{\text {gas }}}$, was allowed to be different from the surface temperature $T$. It was further assumed that the Arrhenius term for adsorption contains the sample temperature $T$. This implies thermal accomodation of the trapped particle, which therefore has the energy $k T$ to overcome the activation barrier $E^{*}{ }_{a d s}$. If we resolve for the pressure $p$, we obtain

$$
p=\frac{\Theta^{n}}{f(\Theta)} \sigma_{A} \sqrt{2 \pi m k T_{g a s}} \frac{v_{n}(\Theta)}{s^{*}} \cdot \exp \left(-\frac{E_{\text {des }}(\Theta)-E^{*}{ }_{a d s}(\Theta)}{k T}\right),
$$

which, for $q_{s t}(\Theta)=E_{d e s}(\Theta)-E^{*}{ }_{a d}(\Theta)$ leads to

$$
\ln p=\ln \left[\frac{\Theta^{n}}{f(\Theta)} \sigma_{A} \sqrt{2 \pi m k T_{\text {gas }}} \frac{v_{n}(\Theta)}{s^{*}}\right]+\frac{-q_{s t}(\Theta)}{R T},
$$


which is formally equivalent to the Clausius-Clapeyron equation. The isosteric heat of adsorption $q_{s t}$ for any given coverage $\Theta$ can be deduced from the slope of a $\ln p$ vs. $1 / T$ plot. The offset term in brackets contains the surface coverage, the kinetic parameters $v_{\mathrm{n}}$ (frequency factor desorption) and $s^{*} / \sqrt{2 \pi m k T}$, which formally corresponds to a frequency factor for adsorption and contains the gasphase temperature $T_{\text {gas }}$. Strong changes in the adsorption kinetics will therefore cause large offsets between the staight lines in the Arrhenius plots. Since this is a purely kinetic derivation, it is evident that no true thermodynamic equilibrium is required (identical gas-phase and sample temperature) for a correct determination of the isosteric heat of adsorption from measured adsorption isotherms or isobars, provided that thermal accomodation of the gas-phase particles prior to adsorption can be assumed.

\subsection{Sticking cofficients}

The initial sticking coefficient $s=s^{*} \exp \left(-E_{\text {ads }}^{*} / k T\right)$ is the ratio between the number of adsorbed particles and the number of impinging particles at vanishing surface coverage, therefore $0 \leq s_{0} \leq 1$. In principle, it can be determined using surface-science techniques capable of measuring absolute coverages upon exposure of the surface to known amounts of gas. Values for $s_{0}$ vary between unity and $10^{-8}$, although for most adsorption systems values between 0.1 and 1 are found [16, 20]. For a particular material, they depend on the surface orientation, and for more open surfaces often have higher values. Also, on atomically rough surfaces with high densities of point defects and steps the sticking coefficient is mostly higher, independent of the particular surface orientation [93-96]. Initial sticking coefficients depend on the population of internal and external degrees of freedom of the impinging molecules, i.e. their translational energy and vibrational and rotational modes [97], which can be investigated via molecular-beam techniques. It turns out that many systems show a "normal energy scaling", which means that the sticking coefficient depends only on the translational energy component normal to the surface [98-104].

In the Langmuir formalism, a linear decrease of the sticking coefficient with surface coverage is assumed for non-dissociative adsorption, $f(\Theta)=(1-\Theta)$. Every particle hitting an occupied surface site is bounced back into the gas-phase. However, many adsorption systems behave differently and exhibit high sticking coefficients upto half monolayer coverages and beyond, then they decrease quite abruptly towards zero. This can be explained by the existence of mobile precursor adsorption states with typical lifetimes in the $10^{-6} \mathrm{~s}$ range. One classifies intrinsic and extrinsic precursor states $[105,106]$. The former exists at empty surface sites and the latter on top of already adsorbed particles. Particles trapped into precursor states are weakly bound to the surface and may be very mobile, so that they can diffuse considerable distances across the surface, until they find an empty adsorption site.

Figure 2.4 is a schematic representation of the possible kinetic processes occuring on a surface with chemisorbed states, as well as intrinsic and extrinsic precursor states. Adsorption from the gas-phase into the precursor states, desorption out of the precursor states and migrations from one precursor into another precursor state occur with certain probabilities. Chemisorption can occur directly or via the intrinsic precursor state. Based on these assumptions, the dependence of the sticking coefficient on the surface coverage was derived by Kisliuk to be [107]:

$$
s(\Theta)=\frac{s_{0}(1-\Theta)}{1+\Theta(K-1)} \text { or } f(\Theta)=\frac{1-\Theta}{1+\Theta(K-1)},
$$

where the constant $\mathrm{K}$ is given by the probabilites indicated in Fig. 2.4:

$$
K=\frac{p_{d}^{\prime \prime}}{p_{c h}^{\prime}+p_{d}^{\prime}}
$$

- 15 -"2kyEi9FYSQjBVrZxIPQ.FHIAC_WRa02_review.doc", Datum: 19.02.03 


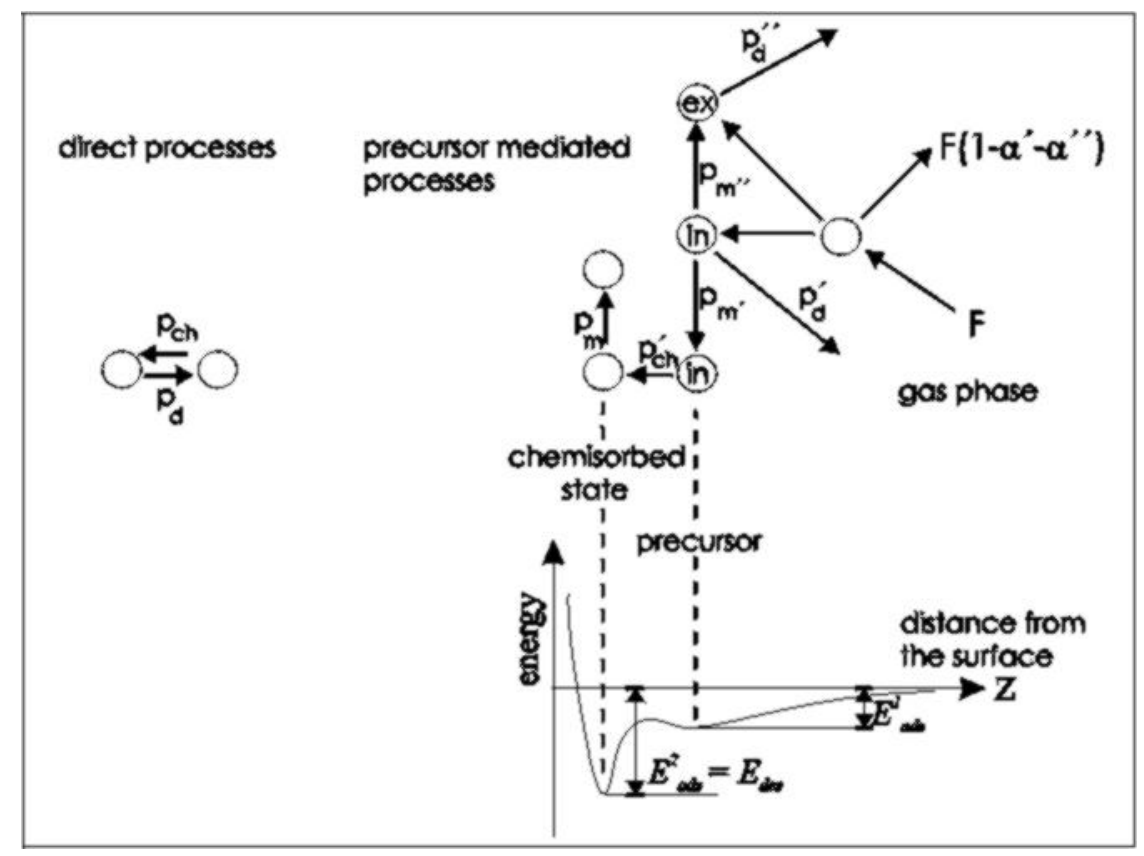

Fig. 2.4. Schematic representation of possible kinetic processes on surface with chemisorption and precursor states. Direct and precursor mediated chemisorption is considered. Processes are correlated with potential energy diagram depicted below and in Fig. 2.1. (ex=extrinsic precursor, in=intrinsic precursor, $\mathrm{F}=$ flux or number of impinging particles, $\alpha^{\prime}$ and $\alpha^{\prime \prime}$ are fractions of trapped molecules, $\mathrm{p}=$ probabilities for migration, desorption or chemisorption)

The coverage-independent sticking coefficient and the linear Langmuir behavior are contained as limiting cases in the Kisliuk model, since $s(\Theta)=s_{0}$ for $K=0$, and $s(\Theta)=s_{0}(1-\Theta)$ for $K=1$. If the probability for desorption out of the extrinsic precursor state $p_{d}^{\prime \prime}$ is smaller than the sum of the probabilities to chemisorb $p_{c h}^{\prime}$ and to desorb out of the intrinsic precursor $p_{d}^{\prime}$, then $K<1$ and a concave curve is obtained (Fig. 2.5). Sometimes $K>1$ and convex curves are observed [108-111]. Coverage dependent sticking coefficients allow to predict more realistic surface coverages at high gas pressures, when chemisorption via the highly populated precursor states becomes the rate limiting step. One has to keep in mind that a particle adsorbed into a mobile precursor state will find a reactive chemisorption site on a catalyst surface, even if this site is located several hundred lattice spacings away.

When the function $f(\Theta)$ according to (2.16) is introduced into (2.10), a more general isotherm (or isobar) equation can be derived for first-order kinetics:

$$
\Theta(p, T)=\frac{1+b_{1}(T) p-\sqrt{\left[1-b_{1}(T) p\right]^{2}+4 K b_{1}(T) p}}{2(1-K)}, \quad K \neq 1 .
$$

The function $b_{l}(T)$ is defined in (2.11). The Langmuir case $(K=1)$ is given by (2.11).

For second-order kinetics, an analytical solution for $\Theta(p, T)$ does not exist. Only for the special case of dissociative adsorption, $f(\Theta)=(1-\Theta)^{2}$, but quasi-first-order desorption $(n=1)$ because of immobile dissociated species, a solution can be derived:

$$
\Theta(p, T)=\frac{1+2 b_{1}(T) p-\sqrt{1+4 b_{1}(T) p}}{2 b_{1}(T) p}
$$




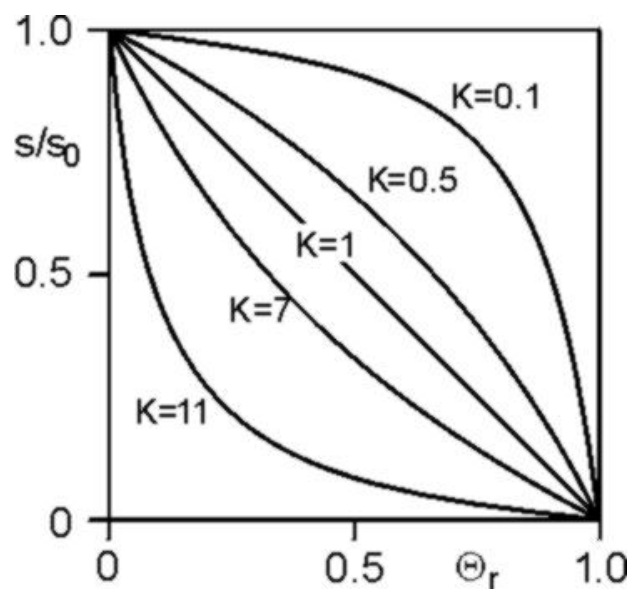

Fig. 2.5. Relative sticking coefficients as function of surface coverage as obtained from Kisliuk model [106, 107]. Parameter $K$ is explained in text.

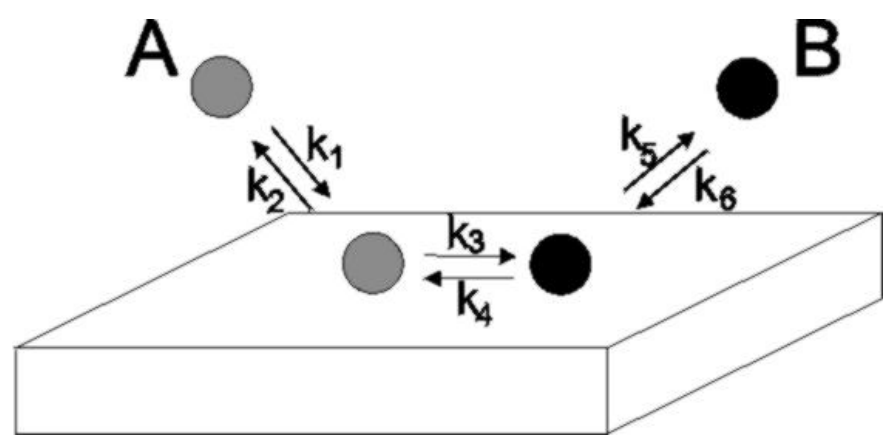

Fig. 2.6. Elementary reaction steps for unimolecular surface reaction.

\subsection{Microkinetic modeling of surface reactions}

Every surface reaction involves at least five elementary steps, as depicted schematically in Fig. 2.6: Trapping and sticking of the reactant(s), adsorption (chemisorption), surface diffusion, chemical reorganization of the reactants (chemical reaction), and desorption of the product(s). Adsorption provides a certain concentration of reactive particles on the surface, which is determined by the gas pressure $p$ and the surface temperature $T$, as discussed in previous sections. The rate of any surface reaction will depend on the reactant surface coverages and, therefore, on these paramaters.

A microkinetic model simulates the rate of a surface reaction for any gas pressure $p$ and catalyst temperature $T$ by using the rates of the elementary steps as input parameters. The modeling can be performed on different levels of complexity [112]. On the most fundamental level, the time development of the system is followed in detail by solving the time-dependent Schrödinger equation or Newton's equation [113]. On the next level, the kinetics can be calculated using the kinetic MonteCarlo method [114-116]. The next level is the continuum description, where the rate of a surface reaction between adsorbed surface species $\mathrm{A} *$ and $\mathrm{B} *$ is assumed to be proportional to their average surface coverages $\Theta_{A}$ and $\Theta_{B}$. The adsorbed species are assumed to be distributed uniformly over the surface and their average properties are considered. Adsorbate-adsorbate interactions are included and the adsorption-desorption parameters depend on the surface coverages. This can lead to quite complex reaction kinetics and kinetic oscillations, if the adsorption probability of one reactant depends strongly on the coverage of another, i.e., if there is a coupling betwen the two adsorbate species $[117,118]$. At the simplest level, in the Langmuir-Hinshelwood description even the coverage dependence of the parameters entering the rate constants is neglected. In the case of bimolecular Langmuir-Hinshelwood reactions, where both reactants are adsorbed, the reactant surface coverages $\Theta_{A}$ and $\Theta_{B}$ are calculated with the Langmuir isotherm formalism for competitive coadsorption. In the case of Eley-Rideal bimolecular reactions, or unimolecular Langmuir-Hinshelwood reactions, the simple Langmuir isotherm can be used for calculating $\Theta_{A}$. Again the reaction rate is assumed to be proportional to the reactant coverages. Although this is a severe simplification, this approach works rather well for several reactions, and is outlined in somewhat more detail below.

First, a reaction mechanism with the elementary steps involved has to be formulated. For a unimolecular surface reaction, one obtains: 
(1)

$\mathrm{A}+$
$\mathrm{A}^{*}$
$\mathrm{~B}^{*}$
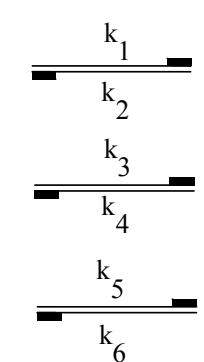

$\mathrm{A}^{*}$

$\mathrm{B}^{*}$

* denotes an empty surface site, which is included as a reactant in the model, A and A* denote the reactant species in the gas-phase and adsorbed onto the surface, respectively. The three elementary steps in (2.21) are described by their corresponding forward and reverse rate constants $k_{1}, k_{2}, \ldots k_{6}$, all of which are given by Arrhenius expressions

$$
k_{i}=v_{i} \exp \left(E_{i} / k T\right)
$$

with activation energies $E_{i}$ and preexponetial frequency factors $v_{i}$. The slowest rate determining step, for example, the surface reaction $\mathrm{A} * \rightarrow \mathrm{B} *$, is described by the rate equation,

$$
r_{2}=k_{2}[\mathrm{~A} *]-k_{3}[\mathrm{~B} *]
$$

where $[\mathrm{A} *]$ and $[\mathrm{B} *]$ correspond to the surface coverages $\Theta_{A}$ and $\Theta_{B}$, respectively. Under thermal equilibrium, this kinetic equation smoothly goes into the corresponding equilibrium equation, and the rate constants are related to the step (2) equilibrium constant $K_{2}$ by $K_{2}=k_{2} / k_{3}$. If all other steps are fast compared to step (2), they can be described by equilibrium equations. As discussed in Section 2.2, step (1) describes the adsorption-desorption equilibrium of the reactant $\mathrm{A}$, which determines the surface coverage $\Theta_{A}$ :

$$
[\mathrm{A} *] /[\mathrm{A}]=\mathrm{k}_{1} / \mathrm{k}_{2}=\mathrm{K}_{1}
$$

or

$$
[A *]=[A] \cdot K_{1}=\Theta_{A}(p, T)=\frac{b_{1}(T) \cdot p}{1-b_{1}(T) \cdot p}, \quad b_{1}(T)=\frac{s^{*} \exp \left[\left(E_{a d s}^{*}-E_{d e s}\right) / k T\right]}{v_{1} \sqrt{2 \pi m k T}} .
$$

The initial sticking coefficient $s_{0}=S^{*} \exp \left(-E^{*}{ }_{a d s} / k T\right)$ and the gas-phase pressure $p$ enter into the rate constant for adsorption $k_{l}$, the frequency factor $v_{l}$ and activation energy for desorption $E_{\text {des }}$ into the rate constant $k_{2}$. Analogous relations hold for the adsorption-desorption equilibrium and the surface coverage of the product B. In the steady-state approximation, the product formation rate $r_{B}$ can be obtained by simple algebra, revealing the Langmuir-Hinshelwood/Hougen-Watson rate law [119],

$$
r_{B}=\frac{\left(\frac{k_{1}}{k_{2}}\right) p_{A} n_{A} \cdot k_{3}-\left(\frac{k_{6}}{k_{5}}\right) p_{B} n_{A} \cdot k_{4}}{1+p_{A}\left(\frac{k_{1}}{k_{2}}\right)+p_{B}\left(\frac{k_{6}}{k_{5}}\right)},
$$

where $n_{A}$ denotes the number of active surface sites. This allows us to calculate the macroscopic reaction kinetics for any pressures and temperatures, based on kinetic and thermodynamic parameters that can be determined from surface science experiments, such as TDS performed under vacuum conditions and lower temperatures, if the number of active sites is known. Such modeling is important for process designing [120], or for simulating surface reactions on model catalyst systems to be compared with quantitative turnover measurements.

Several surface reactions were simulated successfully by microkinetic models [121-124]. In the case of the ammonia synthesis reaction, detailed nitrogen chemisorption studies over Fe single-crystals 
revealed the dissociative chemisorption of $\mathrm{N}_{2}$ as the rate determining step and the parameters for a microkinetic model [117], which was able to correctly extrapolate the catalyst performance to technical reaction conditions [125]. Several comparative experiments over polycrystalline catalyst samples substantiated the model study results and contributed to bridge the "material gap" between single-crystals and polycrystals [126-127]. However, the following problems involved with this extrapolation and with surface-reaction studies on single-crystal model systems in general always have to be kept in mind. The reliability of the extrapolated adsorbate coverages by a Langmuir isotherm formalism and the correct description of adsorbate-adsorbate interactions for the high coverages, under reaction conditions, is still a matter of debate [9]. This approach also assumes the existence of a uniformly composed and structured catalyst surface with identical adsorption sites, which is not necessarily true under reaction conditions. The question of whether the model catalyst used in UHV experiments always really represents the active catalyst state under high pressures with respect to surface structure and composition is a major problem. Technical catalysts are usually complex compounds with several promotor additives in small concentrations, forming a polycrystalline material, which can exhibit several surface planes. In the high pressure gas environment under reaction conditions, the nature of the catalyst can change, the geometric surface structure as a result of the wellknown adsorbate induced restructuring [128-129], the bulk structure and composition, because of solid-state transformations or segregations. These structural changes might not exist under UHV conditions, which is why the "pressure gap" becomes a "material gap". For metal-oxide based catalysts, the material gap can become a more severe problem than for metal catalysts, because a more complex solid-state chemistry determines the catalytically active state on binary compounds.

It may happen, under reaction conditions, that completely new compounds are formed at the catalyst surface or in the subsurface region. This may involve reactant species or other foreign atoms. In this case, the catalyst activity is determined by the properties of these new compounds. In ammonia synthesis over Fe catalysts, the formation of substoichiometric Fe nitrides is discussed [9]. For the partial oxidation of methanol to formaldehyd over copper catalysts the formation of copper suboxides was observed at ambient gas pressures of 10 mbar [130] and on silver catalysts a reactive $\mathrm{O}_{\gamma}$ species embedded into the outermost Ag layer was found [131]. In both cases, these O species differ from adsorbed $\mathrm{O}$ or regular oxide species with respect to their electronic structure and reactivity. Another situation is encountered if the reactants or reactant intermediates form active sites on the surface that completely differ from the catalyst surface, which leads to an autocatalytic mechanism. For example, an active carbonaceous species, carbidic $\mathrm{C}$, was observed to increase the methane formation from synthesis gas $\left(\mathrm{CO}+\mathrm{H}_{2}\right)$ over single-crystal $\mathrm{Ni}(100)$ model catalysts [132, 133].

Despite these problems, the "single-crystal approach" is the first step for gaining insight into the atomic-scale mechanism of heterogeneous catalytic reactions. In the second step, comparative studies on polycrystalline catalyst samples and under elevated gas pressures have to be made, in order to bridge the "material gap". Alternatively. in-situ characterization of the active-catalyst state under technical reaction conditions at elevated gas pressures may be performed, which can be done with single-crystal or polycrystalline systems. Increasing efforts are being put into the development of real in-situ spectroscopic techniques.

\subsection{Preparation of ordered metal-oxide surfaces}

The preparation of metal-oxide surfaces with defined structures and compositions plays a key role in surface-science studies on these materials. The establishment of geometrical order alone is not sufficient, because the stoichiometry on the surface may differ from that of the bulk. Under thermodynamic equilibrium, bulk oxide stoichiometries depend on the $\mathrm{O}_{2}$ gas-phase pressure $p\left(\mathrm{O}_{2}\right)$ and temperature $T$, as given by the corresponding $p\left(\mathrm{O}_{2}\right)-T$ phase diagram. The surface composition will also depend on these parameters. In addition to regular surface areas, atomic defects are always

- 19 -"2kyEi9FYSQjBVrZxIPQ.FHIAC_WRa02_review.doc", Datum: 19.02.03 
present. These can be point defects, such as adatoms or vacancies, atomic steps, domain boundaries, facets, and defects that extend into the bulk, such as grain boundaries. The adsorption properties of defect sites may differ considerably from those of regular surface sites [43], and may play an important role for the catalytic properties of metal-oxides. Since all specroscopic surface-science and reactivity experiments average over large parts of the sample surface, the surface chemistry of defects and regular surface sites must be studied separately. The ultimate goal is to prepare surfaces with different well-defined concentrations of non-ideal defect structures. Such surfaces were prepared successfully by homo- and heteroepitaxy for some metals and bimetals and studied with respect to their adsorption properties [35, 37], for metal-oxides, we are still at the beginning.

When using metal-oxide single-crystals, some surface orientations can be prepared by cleaving or fracture, which certainly produces clean surfaces with the corresponding bulk stoichiometries. An $\alpha$ $\mathrm{Fe}_{2} \mathrm{O}_{3}(0001)$ surface was prepared from a naturally grown crystal in this way [134]. Other orientations have to be prepared by the usual procedure of cutting, polishing, sputtering and annealing in vacuum or in $\mathrm{O}_{2}$ atmosphere. Many metal-oxide surfaces have been prepared in this way as reviewed in [4-6], also among these are several surface planes of $\mathrm{Fe}_{3} \mathrm{O}_{4}$ and $\alpha-\mathrm{Fe}_{2} \mathrm{O}_{3}$ single-crystals [135-141]. However, single-crystal samples may cause several experimental problems. Very often they contain contaminants that segregate to the surface, which makes the preparation of clean surfaces difficult. Sometimes they are wide bandgap insulators, which causes electrostatic charging problems, when using electron-spectroscopy techniques. Their brittle nature makes mounting, heating and temperature control difficult, because spotwelding a thermocouple onto the sample is not possible.

These experimental problems are avoided by using thin epitaxial films that are grown onto conducting metal substrates. Such films also turn out to be very clean if compared to single-crystal samples. Well-ordered films of several transition metal-oxides $(\mathrm{Ni}, \mathrm{Cr}, \mathrm{Fe})$ were prepared by oxidizing the surface region of the corresponding metal single-crystals $[10,142]$, and ordered $\mathrm{Al}_{2} \mathrm{O}_{3}$ films were obtained by surface oxidation of NiAl single-crystals [143].

Another way is to grow heteroepitaxial films onto dissimilar metal substrates, which allows a better control of the film composition and thickness, if the substrate is inert, thus preventing segregation of $\mathrm{O}$ into the underlying substrate, which may be a problem in the case of oxidizing a metal single-crystal surface region. Heteroepitaxial growth was achieved by direct vapor deposition of the oxides for $\mathrm{BaO}$ [144] and $\mathrm{CeO}_{2}[145,146]$. Metal deposition under molecular $\mathrm{O}_{2}$ atmosphere (molecular beam epitaxy, MBE) was applied to oxides of $\mathrm{Zr}$ [147], $\mathrm{Ni}$ [148], Ti [149], Cr [150], V [151], Al, Mg and Si [152, 153]. Plasma assisted MBE was used for oxides of $\mathrm{Mg}, \mathrm{Ni}, \mathrm{Co}, \mathrm{Ti}, \mathrm{Ru}, \mathrm{Mn}, \mathrm{V}, \mathrm{Al}, \mathrm{Cr}, \mathrm{Zr}, \mathrm{Ce}$ and Fe, as revieved recently [154]. A further technique is metal deposition and subsequent oxidation, which was applied to oxides of Ti $[155,156]$, Ce [157], as well as Fe as presented in this work. Ordered Feoxide films were prepared in this way by Vurens et al. for the first time onto $\operatorname{Pt}(111)[158,159]$ and $\operatorname{Pt}(100)$ [160] substrates. Later, the atomic structure of these Fe-oxide monolayer films was studied by STM in conjunction with STM image simulations [161-163] and by photoelectron diffraction experiments [164]. Well-ordered epitaxial Fe-oxide films were also obtained by some other techniques. $\mathrm{Fe}_{3} \mathrm{O}_{4}(100)$ films were grown on $\mathrm{MgO}(100)$ substrates by $\mathrm{MBE}$ in oxygen $[165,166]$ and $\mathrm{NO}_{2}$ [167] atmospheres, several orientations of $\mathrm{Fe}_{3} \mathrm{O}_{4}$ and $\alpha-\mathrm{Fe}_{2} \mathrm{O}_{3}$ films were grown on different surface planes of $\mathrm{Al}_{2} \mathrm{O}_{3}$ and $\mathrm{MgO}$ substrates by oxygen-plasma-assisted $\mathrm{MBE}$ [168-170]. For studying the coupling between magnetic layers, $\mathrm{Fe}_{3} \mathrm{O}_{4} / \mathrm{NiO}$ [171] and $\mathrm{Fe}_{3} \mathrm{O}_{4} / \mathrm{CoO}$ [172] superlattices were grown by MBE.

All these studies revealed that growth parameters, such as $\mathrm{O}_{2}$ gas pressure, metal-deposition rate and substrate temperature are critical for the oxide phases and crystallographic quality of the films. This indicates that the oxidation kinetics plays an important role, which is plausible, because epitaxial growth always occurs under thermodynamical non-equilibrium conditions. Furthermore, it turns out 
that the lattice mismatch between the $\mathrm{O}$ sublattices, in the case of oxide on oxide growth, mainly determines the interfacial energies and, therefore, the epitaxial growth mode.

\subsection{Energetics of metal-oxide surfaces}

The bonding in metal-oxides has both ionic and covalent character, the latter is due to mixing of O2p with metal valence orbitals $(3 \mathrm{~d}, 4 \mathrm{~d} \ldots)$. For a correct description of the surface energetics quantal ab-initio calculations are required, which reveal the complete electronic and geometric surface structure with the lowest total energy. Nevertheless, it is useful to consider the two limiting cases of a purely ionic and a purely covalent bond. This gives a qualitative picture of the surface energetics, which is useful for discussing experimental findings. In a purely ionic model, metal-oxides consist of cations and anions with positive and negative charges. The free energy, and, thus, the stability, is then strongly influenced by electrostatic energies. The surface energy $\gamma_{s}$, defined as the excess energy associated with the surface, is given by [173, 174]:

$$
\gamma_{s}=(\text { cohesive energy of finite crystal) - (number of atoms) }
$$

\section{$\times($ cohesive energy per atom in infinite crystal).}

For purely ionic compounds, it can be determined by calculating Madelung sums, which might become quite complex [175]. A simpler description of the energetics of ionic surfaces, revealing the same results, was introduced by Tasker $[174,176]$. The crystal is considered as a stack of planes, as in Fig. 2.7. An electrically neutral bulk repeat unit, indicated by the brackets in Fig.2.7, which may consist of one or several planes when starting from the surface, builds up the whole crystal, if stacked repeatedly. Three types of surfaces can be classified. For type 1 all planes are equal and have no net charge, because they contain both cations and anions, as, for example, the (100) planes of crystals with the rocksalt structure. The bulk repeat unit consists of one plane only and has no permanent electric dipole moment perpendicular to the surface. If the surface is oriented perpendicular to a polar direction of an ionic crystal, the planes are charged, since they consist only of either anions or cations. For these surfaces, the bulk repeat unit is a composite layer that consist of several charged planes. For a type 2 surface, the oppositely charged planes are arranged in a symmetrical way and again no electric dipole moment perpendicular to the surface exists. An example are the (0001) surfaces of crystals with the corundum structure terminated by a topmost cation layer. For a type 3 surface, the oppositely charged planes generate a permanent dipole moment within the bulk repeat unit, as, for example, (111) surfaces of rocksalt crystals. Note that the classification of type 2 and 3 surfaces depends on the particular surface termination formed. For example, an Fe-terminated $\alpha-\mathrm{Fe}_{2} \mathrm{O}_{3}(0001)$ surface, shown in Fig. 2.7, is of type 2. If the topmost Fe layer is removed, a new bulk repeat unit starting from the surface is obtained, which now has a dipole moment perpendicular to the surface. Thus, an $\mathrm{O}$ terminated $\alpha$ $\mathrm{Fe}_{2} \mathrm{O}_{3}(0001)$ surface represents a type 3 surface.

Surfaces of type 1 and 2 are stable, whereas type 3 surfaces are not. This can be rationalized by considering the electrostatic energy of ions located deep within the bulk of the crystal, if additional composite layers are stacked onto the surface. Since the composite layers of type 1 and 2 surfaces have no electric dipole moment perpendicular to the surface, there is no effect on the potential energy of bulk ions. For a type 3 surface, this potential energy increases monotonically, as depicted in the crystal slab shown Fig. 2.8a. Each bulk repeat unit consists of oppositely charged planes with the distance R and charge densities $\pm \sigma$, which produces a constant electrostatic field $E$ inside the composite layers $\left(E=\sigma / \varepsilon_{0}\right)$, whereas there is no field between them. The electrostatic potential $V$ increases monotonically, leading to an infinite energy for surfaces of type 3 . 
type 1 surface
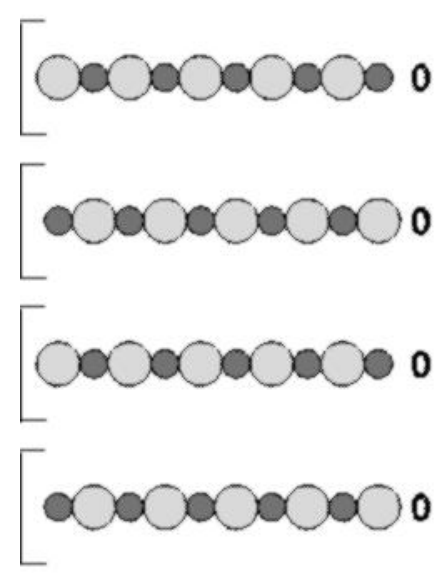

type 2 surface

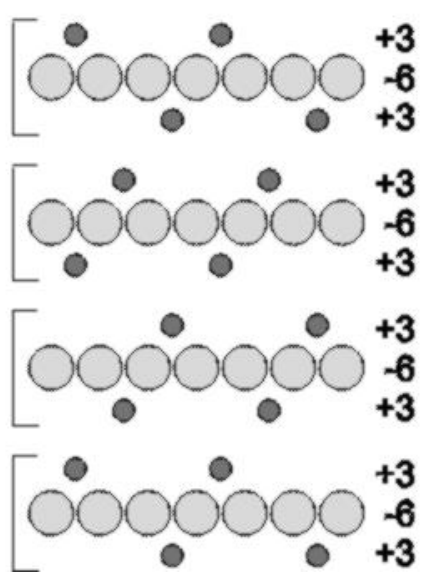

type 3 surface

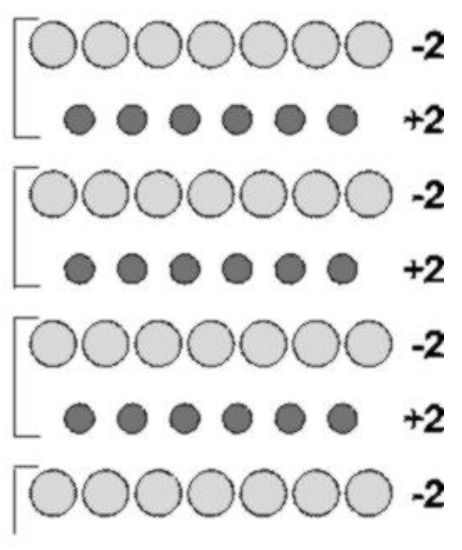

Fig. 2.7. Classification of ionic surfaces exemplified with rocksalt (100) (type 1), corundum (0001) (type 2), and rocksalt (111) (type 3) surfaces. Nominal charges in units of elementary charges per twodimensional unit cell within each layer are given next to atomic planes. Bulk repeat units starting from surface plane are indicated by brackets.

Fig. $2.8 \mathrm{~b}$ shows how an unstable type 3 surface can be transformed into a stable type 2 surface by changing the charge in the surface layers of the crystal slab. When half of the surface charge $-\sigma / 2$ and $+\sigma / 2$ is removed from the left and right layers of the stack, respectively, electrical neutrality of the stack is maintained and a new bulk repeat unit is obtained by formally splitting the layers inside the slab into two parts as indicated by the brackets. Simple electrostatics reveals the potential variation indicated, which now leads to a finite surface energy. The potential energy variations in Fig. 2.8 can also be rationalized by considering the one-dimensional Poisson equation

$$
\frac{d^{2} V(z)}{d z^{2}}=-\frac{\sigma}{\varepsilon \varepsilon_{0}} .
$$

The charge density in each plane determines the curvature of the potential variation $V(z)$ across this plane. At the first plane on the left side in Fig. $2.8 \mathrm{~b}$ this curvature is reduced by a factor of 0.5 , when compared to the corresponding curvature in Fig. 2.8a, leading to the finite potential throughout the crystal. Thus, a type 3 surface can be stabilized by a reduced surface charge.

A reduction of the surface charge can be realized in several ways:

- by removing ions from the topmost surface layer thereby creating vacancies;

- by reconstructions involving several layers. An octopolar reconstruction was predicted for rocksalt (111) surfaces [177, 178] and observed experimentally on $\mathrm{NiO}(111)$ [ 10];

- by adsorption of charged species, such as protons or $\mathrm{OH}$ groups, as observed on $\mathrm{NiO}(111)$ and $\mathrm{Cr}_{2} \mathrm{O}_{3}(0001)$ surfaces [64];

- by strong surface relaxations that change the electronic density of states at the surface, which can also reduce or even compensate the excess surface charge. This mechanism is assumed to happen at the polar $\mathrm{Fe}_{3} \mathrm{O}_{4}(111)$ surface studied here. This certainly is one driving force for the large relaxations observed at many polar metal-oxide surfaces, both experimentally [179, 180] and theoretically [180-182]. 
a)

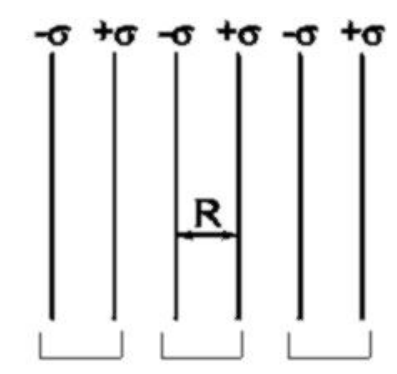

b)

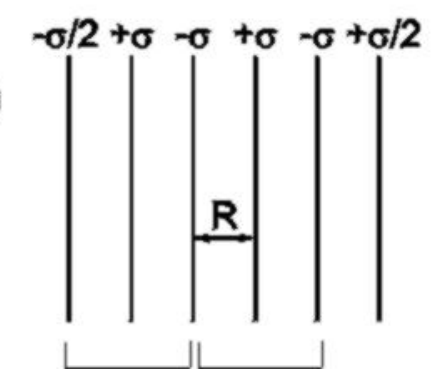

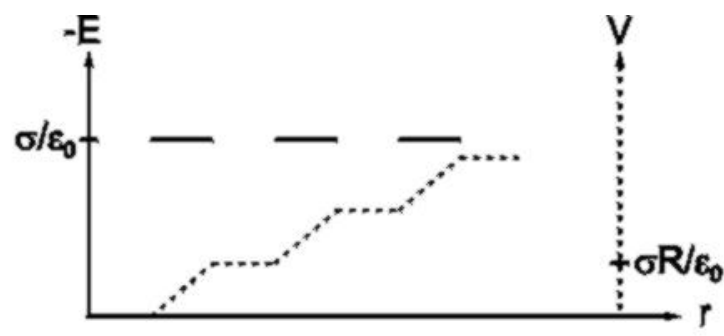

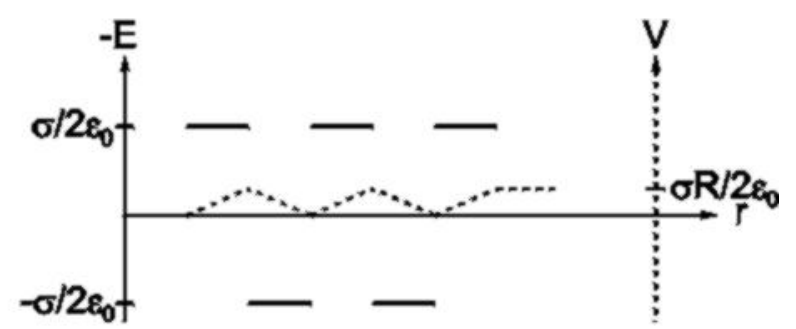

Fig. 2.8. Spatial variation of electric field $E$ (full lines) and electrostatic potential $V$ (dashed lines) in crystal along polar direction. Bulk repeat units indicated by brackets consist of planes separated by distance $R$ and bearing charge densities $\pm \sigma$. (a) shows type $3 \quad$ surface where electrostatic potentia increases monotonically through crystal and surface energy becomes infinite. (b) shows type 2 surface that was obtained from type 3 surface by removing half of surface plane charges. Here electrostatic potential and surface energy remain finite.

In the covalent limit, the energetically favored surface is the one with the minimum number of broken bonds (so-called dangling bonds) at the surface. The minimization of the number of dangling bonds is a well-known driving force for reconstructions on semiconductor surfaces, as for example, the dimer formation on the $\operatorname{Si}(100)-(2 \times 1)$ surface $[183,184]$. For a simple counting of dangling bonds, the different bonds need to be weighted according to their coordination numbers. For example, the breaking of a tetrahedral bond should require about 1.5 times the energy of the breaking of an octahedral bond, assuming an identical number of valencies in both cases. For metal-oxides containing several types of ions that are differently coordinated, the number of dangling bonds depends on the particular surface termination. The $\mathrm{Fe}_{3} \mathrm{O}_{4}(111)$ type 3 surface was found to form a strongly relaxed bulk termination, which among all possible bulk terminations has the smallest number of dangling bonds, as well as the bulk repeat unit with the smallest electric dipole moment [185].

\section{Experimental}

\subsection{Instrumentation}

\subsubsection{Surface analysis systems}

Four UHV chambers, with different combinations of surface analytical tools, were used in the studies reviewed here. All chambers were pumped by turbomolecular pumps yielding a base pressure below $10^{-10}$ mbar. In order to avoid vibrations, the STM chamber contained an additional ion pump. The turbo pump could be separated by a gate valve and switched off during the measurements. All chambers were equipped with a gas inlet manifold, evaporators for $\mathrm{Fe}$ and potassium, a sputter gun and full rotatable manipulators with almost identical sample heating-cooling stations. A small cell for high- 
pressure treatments is attached via a $63 \mathrm{~mm}$ gate valve to all chambers. It also serves as load lock. The samples can be transferred using a magnetically coupled transfer rod.

Two chambers were connected by a gate valve and the sample could also be transferred between them. One of them contains an ion-scattering spectroscopy (ISS) spectrometer and a photoelecton emission microscope (PEEM). The other is mainly used for TDS experiments. A Balzers quadrupole mass spectrometer (QMS) is mounted horizontally and the sample can be placed $1 \mathrm{~mm}$ in front of a stainless steel tube with a diameter of $5 \mathrm{~mm}$, smaller than the sample diameter of $10 \mathrm{~mm}$. This tube is connected to the ionization region of the QMS, which is shielded from the surrounding by a tantalum basket, so that only molecules desorbing from the sample surface are detected. This chamber also contains a backview LEED optics and a cylindrical mirror analyser (CMA) for AES. The STM analysis chamber is equipped with a commercial Burleigh Instruments STM head mounted horizontally on a $150 \mathrm{~mm}$ flange, a backview LEED optics and a CMA Auger spectrometer. The STM and ISS/PEEM chambers are mounted on tables with pneumatic air legs for vibration damping. The final chamber contains a double pass CMA for photoelectron spectroscopy using UV light from a He discharge lamp (UPS) of X-rays from a $\mathrm{Mg} \mathrm{K} \alpha / \mathrm{Al} \mathrm{K} \alpha$ source (XPS), a QMS and a high-resolution LEED optics suitable for spot profile analysis work (SPALEED).

\subsubsection{Sample transfer and heating}

Figure 3.1a shows a front view photograph of the manipulator head with the sample heating-cooling station and the sample on its holder, pushed half-way into the sample quiver [186]. Figure 3.1b is a schematic side view in the transfer position with the transfer rod and wobble stick. The magnetic rod is connected to the manipulator by the indicated centering bores, which provides mechanical stability during transfer. Using the wobble stick, the sample holder can be pulled to the transfer rod, where it is held by spring clamps, or it can be pushed from the transfer rod into the sample quiver on the manipulator. Afterwards, the wobble stick and the transfer rod are retracted. Due to the mechanical connection between manipulator and transfer rod, the transfer is safe and a sample loss is not possible.

The sample holder is fabricated from a sapphire single-crystal. Sapphire was chosen because it has a high thermal conductivity below room temperature allowing fast liquid nitrogen cooling, whereas its thermal conductivity is low at elevated temperatures, thus lifting the thermal contact during annealing. Sapphire is mechanically stable and generates no trouble during sample transfer. It is chemically inert, which makes it suitable for prolonged oxidation treatments at elevated pressures and catalytic experiments in reactive atmospheres. Heating from 100 to $1300 \mathrm{~K}$ with $5 \mathrm{~K} / \mathrm{s}$ does not damage the sapphire sample holder, if it was annealed at $2100 \mathrm{~K}$ before. It also provides electrical insulation needed for applying bias voltages to the sample during STM and photoemission measurements. In the front view photograph (Fig. 3.1a) of the sample heating-cooling station, the sample holder with a mounted Pt sample can be seen. Below the sample the sapphire holder has a $8 \mathrm{~mm}$ diameter hole, so that electron beam heating from behind is possible, when the sample holder is placed into its final position on the manipulator. The filament of the electron beam heater is placed within a ceramic tube and can be seen in Fig. 3.1a. Sample temperatures up to $1500 \mathrm{~K}$ are reached with this heater. Four holes around the sample are used for screws that provide electrical contact with spring bolts on the manipulator station. Two screws provide electrical contact to a thermocouple spotwelded to the side of the sample. Two screws are used to fix the sample either by Pt clamps or by tungsten wires. Currents up to $30 \mathrm{~A}$ can be passed through, which allows for resistive sample heating, if wanted when tungsten wires are used. Temperatures up to $1000 \mathrm{~K}$ are reached with this wire heater. The manipulator head contains a liquid nitrogen reservoir made from copper for sample cooling down to $100 \mathrm{~K}$. 

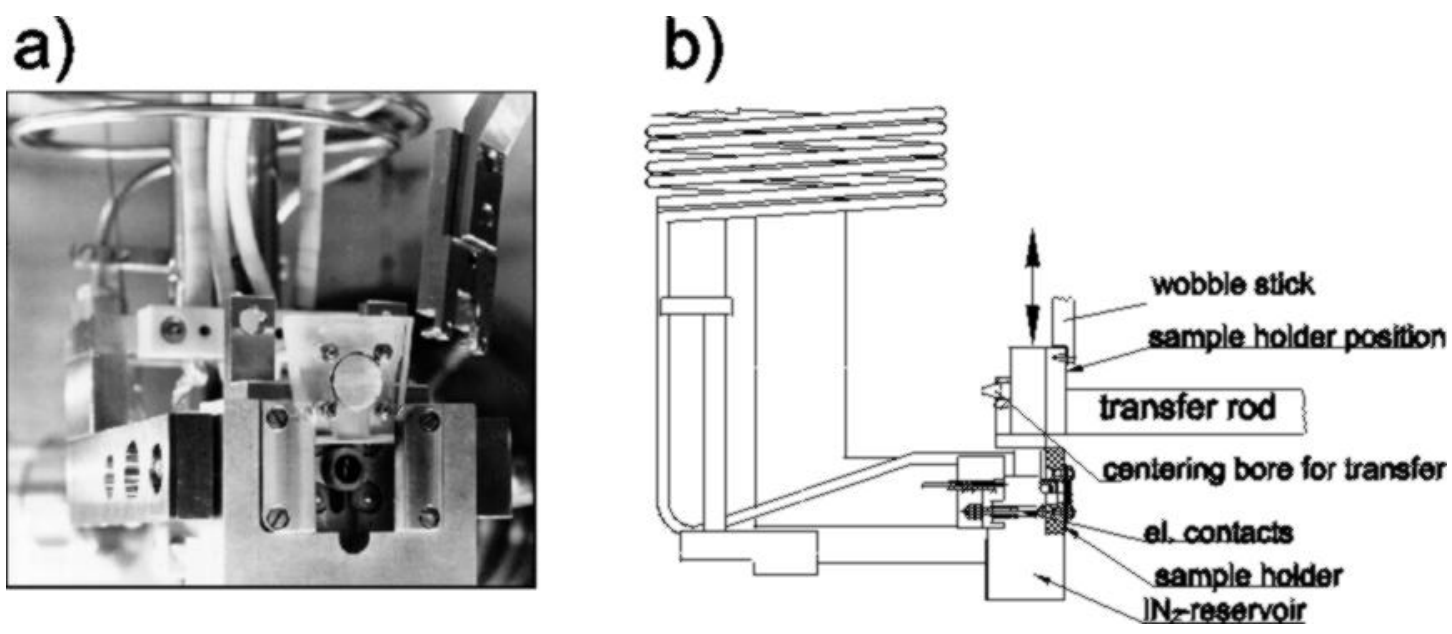

Fig. 3.1: (a): Front view photograph of sample heating-cooling station on the off-axis manipulator (a). (b) Schematic side view of magnetic transfer rod, wobble stick, and heating-cooling station in transfer position.

\subsubsection{High pressure reaction cells}

The high pressure cells consist of $63 \mathrm{~mm}$ diameter double crosses, which are separated from the main chambers by $63 \mathrm{~mm}$ gate valves. The sample is transferred in the same way from the magnetic rods into the sample stations of the high pressure cells, as described in the previous section. The sample station is similar to that on the manipulators. Since electron beam heating is not possible at high pressures, the resistive heater is used for short oxidation treatments up to $\mathrm{O}_{2}$ pressures of 1 mbar. For longer oxidation treatments in $\mathrm{O}_{2}$ pressures up to 1000 mbar, a $250 \mathrm{~W}$ halogen lamp can be positioned in front of the sample and used as a radiation heater. Temperatures up to $1000 \mathrm{~K}$ can be reached in this way. For batch reactor experiments, the educt gases are leaked into the cell and a stainless steel cup is put over the sample using the wobble stick, thus forming a batch reactor with approximately $1 \mathrm{ml}$ volume. For product detection during the batch reactor experiments, reactor gas is leaked via a stainless steel tube from the cup directly into the ionization region of the QMS in the neighboring TDS chamber. At total reactor pressures between 0.1 and 10 mbar, this allows online product and educt detection [186].

\subsection{Spectroscopic and micoscopic methods}

\subsubsection{STM, LEED, TDS and PEEM}

All STM measurements were performed at room temperature in the constant current mode. Tungsten tips were etched electrochemically in 3-molar $\mathrm{NaOH}$ solution and placed afterwards into the STM magazine. Before performing the measurements the tips were cleaned in situ by electron bombardment. Calibration of the scanner was performed on a $\mathrm{Si}(111)-(7 \times 7)$ surface. Image processing of the experimental data included background subtraction and smoothing procedures, some images were corrected for thermal drifts.

The LEED measurements were performed at $T=120 \mathrm{~K}$. Intensity-voltage curves were measured under normal incidence. The diffraction patterns were recorded with a CCD camera from the LEED screen and stored via a VMEbus computer onto a workstation hard disk (ultra sparc 2). Noise reduction was possible by averaging up to 255 images. For extraction of the $I-V$ curves, these images were processed with software programs developed at the Fritz-Haber-Institut. 
For all TDS experiments, the gases were adsorbed at $\mathrm{T}=100 \mathrm{~K}$, if not stated otherwise. The heating rate was always $5 \mathrm{~K} / \mathrm{s}$, and the exposures are given in Langmuirs $\left(1 \mathrm{~L}=1.33 \times 10^{-6} \mathrm{mbar}\right)$. For the PEEM measurements the sample is illuminated with xenon or deuterium discharge lamps. PEEM measurements could be performed at different sample temperatures.

For the adsorption and batch reactor experiments EB and water were dosed via leak valves into the UHV chambers or into the high pressure cells, respectively. Triple destilled water and high purity EB were used. The EB was filled under $\mathrm{N}_{2}$ atmosphere into small glass bulbs containing activated molecular sieve to reduce possible contaminations by water. Before dosing water or EB into the chambers the gas lines were pumped off and backfilled again in order to remove possible contaminations. The pressure indication of the ionization gauge was corrected for the ionization probability of EB, which is about 7 times higher than for $\mathrm{N}_{2 \cdot}$ [187]

\subsubsection{XPS, UPS and XAS}

Ultraviolett photoelectron spectroscopy (UPS) using a Helium resonance lamp (He I line $21.2 \mathrm{eV}$, He II line, $40.8 \mathrm{eV}$ ) and $X$-ray photoelectron spectroscopy (XPS) using $\mathrm{MgK} \alpha$ radiation ( $h v=1253.6$ $\mathrm{eV}$ ) were performed using a double pass CMA. The direction of light incidence was normal to the analyser axis. As a result, the spectra were averaged over a large range of escape angles.

$X$-ray adsorption spectroscopy (XAS) was performed with different systems at BESSY-I. They also allowed to perform conventional XPS measurements. Details will be given together with the spectra to be presented. The photon energy was calibrated to an accuracy of $\pm 0.5 \mathrm{eV}$ by reference to the $\mathrm{La3d} \rightarrow 4 \mathrm{f}$ transition at $836 \mathrm{eV}$. All XAS data were collected in the partial electron yield modus $(-450$ $\mathrm{eV}$ retarding voltage), resulting in a probing depth comparable to XPS. All spectra of clean and adsorbate covered Fe-oxide films were divided by the spectrum from the clean $\operatorname{Pt}(111)$ sample or a clean Au surface, which eliminated spectral artifacts from contaminated optical components.

\subsection{Preparation of epitaxial iron-oxide films}

Since heteroepitaxial growth offers the best control of film stoichiometry, thickness and morphology, single-crystalline Fe-oxide films were grown on $\mathrm{Pt}(111)$ substrates. The Pt(111) surface was cleaned by cycles of argon ion bombardment $(1 \mathrm{keV})$ and annealing to $1300 \mathrm{~K}$ and by oxidationannealing cycles, until it exhibited a sharp LEED pattern and no contamination signals in AES. The Fe-oxide films were prepared by Fe deposition at room temperature and subsequent oxidation for at least 2 min at temperatures between 870 and $1000 \mathrm{~K}$ in $10^{-6} \mathrm{mbar}_{2}$ partial pressure as shown in Fig. 3.2. In this way, 1-2 monolayer thick $\mathrm{FeO}(111)$ films and $\mathrm{Fe}_{3} \mathrm{O}_{4}(111)$ multilayer films can be prepared. $\alpha-\mathrm{Fe}_{2} \mathrm{O}_{3}(0001)$ films were obtained by oxidizing $\mathrm{Fe}_{3} \mathrm{O}_{4}$ films in $\mathrm{O}_{2}$ pressures between $10^{-4}$ and 1 mbar in the seperate high pressure cells. After this oxidation, the samples were cooled down to room temperature in the high pressure $\mathrm{O}_{2}$ atmosphere, then the $\mathrm{O}_{2}$ was pumped off and the sample was transferred back into the analysis chamber. In the STM chamber, the Fe evaporation is accomplished with an electron beam evaporator, in all other chambers by resistively heating a tungsten wire with an Fe wire wrapped around it. In the STM chamber, the Fe-oxide film thickness was calibrated using STM by determining the amount of Fe necessary to complete Fe-oxide monolayers. In the UPS chamber, the Fe deposition rate was estimated from the first oxide layer formation. Since EB adsorbs on $\mathrm{Pt}(111)$, but not on the thin initial $\mathrm{FeO}(111)$ film at room temperature, the first $\mathrm{FeO}$ layer coverage can be titrated by the characteristic UPS valence-band emission features of EB adsorbed onto Pt(111). A corresponding titration was also performed using TDS of CO, which adsorbs on Pt(111), but not on the $\mathrm{FeO}$ film. 


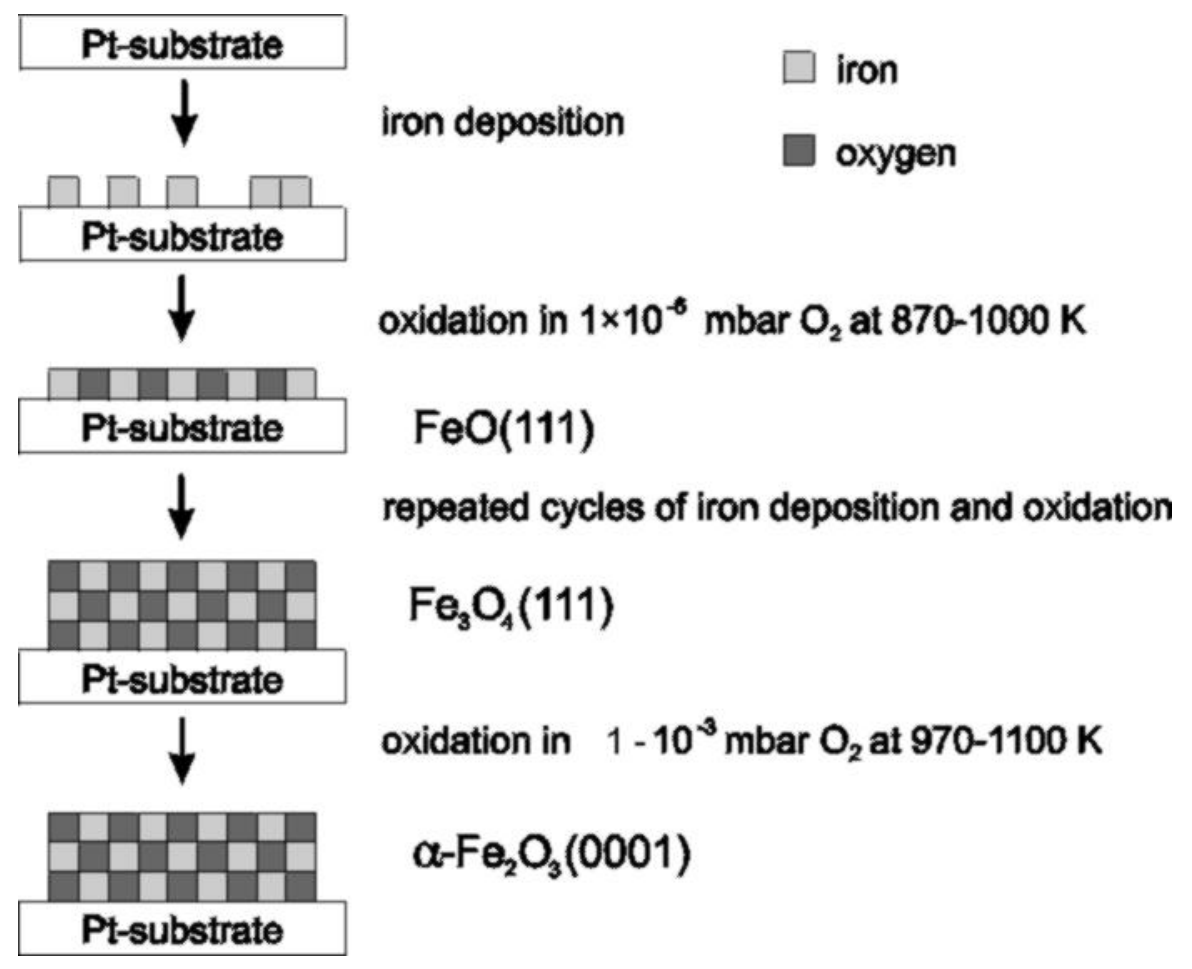

\section{Styrene synthesis}

\subsection{Thermodynamics and byproducts}

The dehydrogenation of $\mathrm{EB}$ to $\mathrm{St}$ is an endothermic $(\Delta H=129.4 \mathrm{~kJ} / \mathrm{mol})$ and reversible reaction [188]:

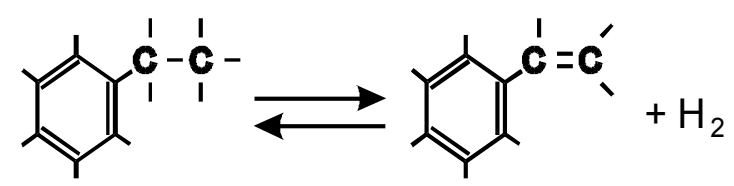

At room temperature, the reaction equilibrium is located far towards the educt side. It can be shifted towards the product side by increasing the temperature, which increases the equilibrium constant $\mathrm{K}$, due to the van't Hoff relationship and by reducing the pressure, since two moles of product are formed from one mol of EB. Therefore, the technical styrene synthesis is run around $600^{\circ} \mathrm{C}$ with an excess of steam with water-EB mixtures with molar ratios from 5:1 to 12:1. Styrene plants run their reactors under isothermal or adiabatic conditions with flow rates that ensure short contact times in order to prevent polymerization of styrene [12]. The equilibrium $\mathrm{EB}$ conversion at $600^{\circ} \mathrm{C}$ and 0.1 bar pressure is $83 \%$ [189], and conversions between 50 and $60 \%$ are obtained in technical reactors. The main byproducts of the EB dehydrogenation are benzene $(\approx 1 \%)$ and toluene $(\approx 2 \%)$ formed by catalytic dealkylation and hydrodealkylation of $\mathrm{EB}$, respectively:

$$
\begin{aligned}
\mathrm{C}_{6} \mathrm{H}_{5} \mathrm{CH}_{2} \mathrm{CH}_{3} & \rightleftarrows \mathrm{C}_{6} \mathrm{H}_{6}+\mathrm{C}_{2} \mathrm{H}_{4} \\
\mathrm{C}_{6} \mathrm{H}_{5} \mathrm{CH}_{2} \mathrm{CH}_{3}+\mathrm{H}_{2} & \rightleftarrows \mathrm{C}_{6} \mathrm{H}_{5} \mathrm{CH}_{3}+\mathrm{CH}_{4}
\end{aligned}
$$

They also can be formed by steam dealkylation:

$$
\begin{array}{ll}
\mathrm{C}_{6} \mathrm{H}_{5} \mathrm{CH}_{2} \mathrm{CH}_{3}+\mathrm{H}_{2} \mathrm{O} \rightleftarrows \mathrm{C}_{6} \mathrm{H}_{5} \mathrm{CH}_{3}+\mathrm{CO}+2 \mathrm{H}_{2} \\
\mathrm{C}_{6} \mathrm{H}_{5} \mathrm{CH}_{2} \mathrm{CH}_{3}+2 \mathrm{H}_{2} \mathrm{O} \rightleftarrows \mathrm{C}_{6} \mathrm{H}_{6}+2 \mathrm{CO}+3 \mathrm{H}_{2}
\end{array}
$$


All these reactions are accompanied by the formation of coke that can deactivate the catalysts. This coke is removed by combustion with steam according to the water-gas shift reaction:

$$
\begin{array}{lll}
\mathrm{C}+\mathrm{H}_{2} \mathrm{O} & \rightleftarrows \mathrm{CO}+\mathrm{H}_{2} \\
\mathrm{CO}+\mathrm{H}_{2} \mathrm{O} & \rightleftarrows \mathrm{CO}_{2}+\mathrm{H}_{2}
\end{array}
$$

The dilution of EB with superheated steam, therefore, has several desired effects for the styrene synthesis:

- It increases the EB conversion, because the educt partial pressure is reduced.

- It supplies part of the heat needed for the endothermic reaction.

- It removes carbonaceous deposits from the catalyst surface.

- Since $\mathrm{H}_{2}$ is formed during EB dehydrogenation, steam prevents the reduction of Fe-oxide to metallic Fe. It stabilizes an active Fe-oxide stoichiometry under reaction conditions.

\subsection{Technical catalysis}

The industrial dehydrogenation of EB to styrene has been carried out over potassium promoted Feoxide catalysts since 1957 [188]. Today, about 23 million tons of styrene are produced per year worldwide [190], which makes even small improvements of the catalysts profitable. An extensive review on the development of styrene synthesis catalysts was published by Lee [191], a recent review can be found in [12]. Potassium increases the activity of pure $\mathrm{Fe}_{2} \mathrm{O}_{3}$ catalysts by one order of magnitude, and is believed to remove carbonaceous surface deposits by catalyzing the combustion of coke with steam [191, 192]. Technical catalysts are prepared from about $80 \mathrm{wt} \%$ of Fe-oxide and at least $10 \mathrm{wt} \%$ of potassium oxide. Small amounts of alumina and chromia act as structural promotors and increase the lifetime of the catalysts [189]. Oxides of V, Ce, W or Mo improve the selectivity, but the effect is only moderate [193]. Therefore, any catalyst model can be restricted to systems consisting of $\mathrm{Fe}$ and potassium oxides.

After starting the catalytic reaction in an EB-steam mixture, the EB conversion to St increases gradually. The steady state is reached within 20 hours time on stream [193-195], which indicates formation of the active phase under reaction conditions. Mross proposed the working catalyst to consist of $\mathrm{Fe}_{2} \mathrm{O}_{3}, \mathrm{~K}_{2} \mathrm{Fe}_{22} \mathrm{O}_{34}$, and $\mathrm{Fe}_{3} \mathrm{O}_{4}$ covered by a liquid film of $\mathrm{KOH}$ [196], a model that was ruled out later. Hirano first proposed an active $\mathrm{KFeO}_{2}$ phase on the catalyst surface, based on X-ray diffraction measurements on spent and "steamed" catalysts in the absence of air [194]. This was substantiated later by Muhler et al., who performed a detailed study of the catalyst precursor and of the working catalyst applying a variety of in-situ and ex-situ techniques [195, 197]. They developed the catalyst life-cycle shown schematically in Fig. 4.1.

The precursor material consists of hematite, $\alpha-\mathrm{Fe}_{2} \mathrm{O}_{3}$, and the ternary $\mathrm{K}_{2} \mathrm{Fe}_{22} \mathrm{O}_{34}$ phase, which has a cubic crystal structure similar to the spinel structure of $\mathrm{Fe}_{3} \mathrm{O}_{4}$, magnetite. Under reaction conditions, the active catalyst phase is formed, which consists of a thin $\mathrm{KFeO}_{2}$ layer supported on a solid solution of $\mathrm{K}_{2} \mathrm{Fe}_{22} \mathrm{O}_{34}$ in $\mathrm{Fe}_{3} \mathrm{O}_{4}$. The $\mathrm{K}_{2} \mathrm{Fe}_{22} \mathrm{O}_{34}$ phase acts as a storage medium from which the active surface is continuously supplied with a near monolayer coverage of potassium ions. This potassium migration causes a continious solid-state transformation of the catalyst during its lifetime. An irreversible deactivation is caused by spatial disintegration of the Fe-oxide and the potassium compound leading to a core of potassium rich $\mathrm{Fe}_{3} \mathrm{O}_{4}$ and a shell of only $\mathrm{Fe}_{3} \mathrm{O}_{4}$, by the total loss of potassium, and by the reduction of $\mathrm{KFeO}_{2}$ and $\mathrm{K}_{2} \mathrm{Fe}_{22} \mathrm{O}_{34}$ into $\mathrm{Fe}_{3} \mathrm{O}_{4}$ and $\mathrm{KOH}$. A reversible deactivation of the catalyst is caused by blocking of active surface sites with $\mathrm{CO}_{2}$ adsorbed in competition with the reactant, as well as by the formation of inactive carbonaceous surface deposits. These species can be removed by a 
steam treatment. A catalytically active role of the carbonaceous species has never been discussed in literature for the non-oxidative dehydrogenation process.

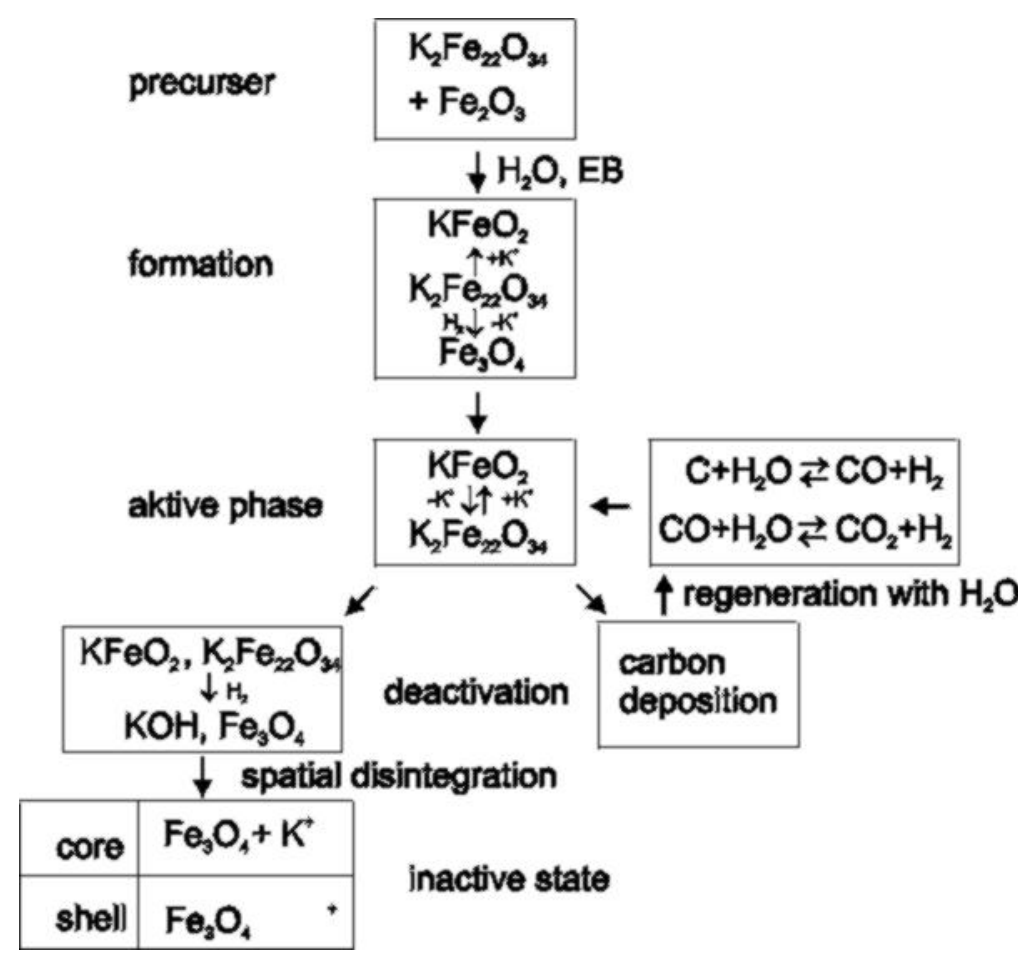

Fig. 4.1. Schematic life cycle of styrene catalyst with potassium and no other promotor additives [195].

\subsection{Reaction kinetics and mechanism}

The first kinetic studies of EB dehydrogenation was performed by Carra and Forni [198]. They desribed the reaction by a unimolecular Langmuir-Hinshelwood mechanism, where the reaction rate depends on the adsorption-desorption equilibrium of EB and styrene. The stronger adsorption of styrene leads to a site-blocking effect by the product. These findings were confirmed by further studies of Lebedev et al. [199], Hirano [193, 194], and Goodman et al. [192]. It was established that water vapor does not participate in the reaction itself, but acts purely as inert dilution agent. However, as discussed above, it prevents the formation of inactive carboneceous surface deposits and the reduction of the Fe-oxide to metallic Fe by the produced $\mathrm{H}_{2}$. Whereas Carra and Forni [198] assume one type of active sites generating all products in parallel reactions, several specialized reaction sites were postulated by Hirano [194]. For potassium promoted catalysts, activation energies ranging between 120 and $190 \mathrm{~kJ} / \mathrm{mol}$ were found. Since the activation energies for unpromoted $\alpha-\mathrm{Fe}_{2} \mathrm{O}_{3}$ catalysts were similar $(150$ to $170 \mathrm{~kJ} / \mathrm{mol})$ [192, 194, 198, 200] identical active sites for the styrene formation on potassium promoted and unpromoted Fe-oxide catalysts were proposed [194, 201].

According to [197], the active catalyst exposes a surface containing $\mathrm{Fe}^{3+}$ ions and $\mathrm{K}^{+}$ions at a ratio of 1:1, which was already suggested in Lee's model [191]. In analogy to the oxidative dehydrogenation of butadiene over $\mathrm{MgFe}_{2} \mathrm{O}_{4}$ catalysts [202], a two step mechanism associated with an acidic and a basic site is postulated for the dehydrogenation of EB over Fe-oxide and other transition metal-oxide catalysts [197, 203]. Such a reaction scheme was proposed previously [204]. The C-H groups of the EB-ethylgroup are deprotonated at basic $\mathrm{O}$ sites and two $\mathrm{OH}$ groups are formed at the surface. Simultaneously, or subsequently, an electron transfer to acidic $\mathrm{Fe}^{3+}$ sites is required before the styrene product molecule can desorb from the catalyst surface. Finally, the $\mathrm{H}$ atoms forming the two $\mathrm{OH}$ groups somehow leave the catalyst surface, the reduced $\mathrm{Fe}^{2+}$ species become reoxidized to $\mathrm{Fe}^{3+}$, and 
the basic $\mathrm{O}$ sites are reestablished for the next turnover cycle. This picture agrees with results from an infrared spectroscopy study, where styrene formed by EB dehydrogenation was observed to be bound to the catalyst surface through the vinyl group, whereas styrene adsorbed from the gas-phase was bound via the aromatic ring [201]. In the active $\mathrm{KFeO}_{2}$ surface phase, potassium saturated $\mathrm{Fe}-\mathrm{O}$ bonds increase the basicity of the $\mathrm{O}$ sites. They also must be located in an appropriate geometry with respect to the acidic $\mathrm{Fe}^{3+}$ sites, so that an effective deprotonation of the $\mathrm{EB}$ becomes possible.

\subsection{Oxydehydrogenation of ethylbenzene over carbon-based catalysts}

Since the early seventies, it is known that certain types of carbon show good catalytic activities and selectivities for the oxydehydrogenation of EB to styrene [205]:

$$
\mathrm{C}_{6} \mathrm{H}_{5} \mathrm{CH}_{2} \mathrm{CH}_{3}+1 / 2 \mathrm{O}_{2} \rightleftarrows \mathrm{C}_{6} \mathrm{H}_{5} \mathrm{C}_{2} \mathrm{H}_{3}+\mathrm{H}_{2} \mathrm{O} \text {. }
$$

Since then there has been a great deal of interest in oxidative dehydrogenation schemes [206-209], because the formation of water as a byproduct makes the process exothermic and thermodynamically enables complete conversion. This also reduces the energy consumption for the styrene synthesis over Fe-oxide catalysts considerably. In more recent studies, various carbon materials including zeolithes exhibited higher activities and selectivities than Fe-oxide based catalysts at much lower reaction temperatures than $600^{\circ} \mathrm{C}[210,211]$. For example, $80 \%$ conversion and $90 \%$ selectivity were achieved at $350^{\circ} \mathrm{C}$ over ultrahigh surface area carbon molecular sieves [212].

During the 1980's, the oxydehydrogenation of EB was investigated over various oxide compounds $[213,214]$ and phosphates by several groups [215-218]. It was found that carbonaceous layers form on the surface of these compounds, which act as the true catalyst. This "active coke" is an organic polymer that is formed from EB and $\mathrm{O}_{2}$ in the feed and contains $\mathrm{C}, \mathrm{H}$ and O. XPS and SIMS measurements indicated the presence of carbonyl species and quinone-like groups $[219,220]$. The $\mathrm{O}$ atoms in these quinoid groups can dehydrogenate EB forming water as a product, as proposed by Emig and coworkers in a speculative reaction mechanism [215]. About 50\% of the surface is supposed to be covered by these carbonaceous species, after an equilibrium between their formation and combustion is established. The formation of this acive coke was found to depend on the surface acidity of the inorganic support [221, 222]. Basic substrates, such as magnesia and titania are nearly inactive, and very acidic sites are also ineffective $[215,223]$. Generally, sites with a Lewis acidity of moderate strength are considered to form the active coke most effectively [218]. So far there never was evidence that carbonaceous overlayers act as the true catalyst for the dehydrogenation of ethylbenzene over Feoxide based catalysts.

\section{Growth and characterization of epitaxial iron-oxide films}

\subsection{Iron-oxide crystal structures and stability ranges}

$\mathrm{Fe}$ and $\mathrm{O}$ form a number of phases with different stoichiometries and crystal structures. These are: $\mathrm{FeO}$ (wüstite), $\mathrm{Fe}_{3} \mathrm{O}_{4}$ (magnetite), $\alpha-\mathrm{Fe}_{2} \mathrm{O}_{3}$ (hematite), $\gamma-\mathrm{Fe}_{2} \mathrm{O}_{3}$ (maghemite) and $\varepsilon-\mathrm{Fe}_{2} \mathrm{O}_{3}$ [224]. The latter two phases have only been synthesized artificially, but all other phases occur also naturally. Under thermodynamic equilibrium, their stability ranges depend on the ambient $\mathrm{O}_{2}$ gas pressure $p\left(\mathrm{O}_{2}\right)$ and on the temperature $T$. These stability ranges for pressures below 1 bar are shown in the phase diagram for the Fe-O system [225] depicted in Fig. 5.1. It was calculated using commercial software [226] for $\mathrm{p}\left(\mathrm{O}_{2}\right)$-T ranges used in the preparation of the Fe-oxide films. It agrees well with $\mathrm{Fe}-\mathrm{O}_{2}$ phase diagrams published earlier [227]. 


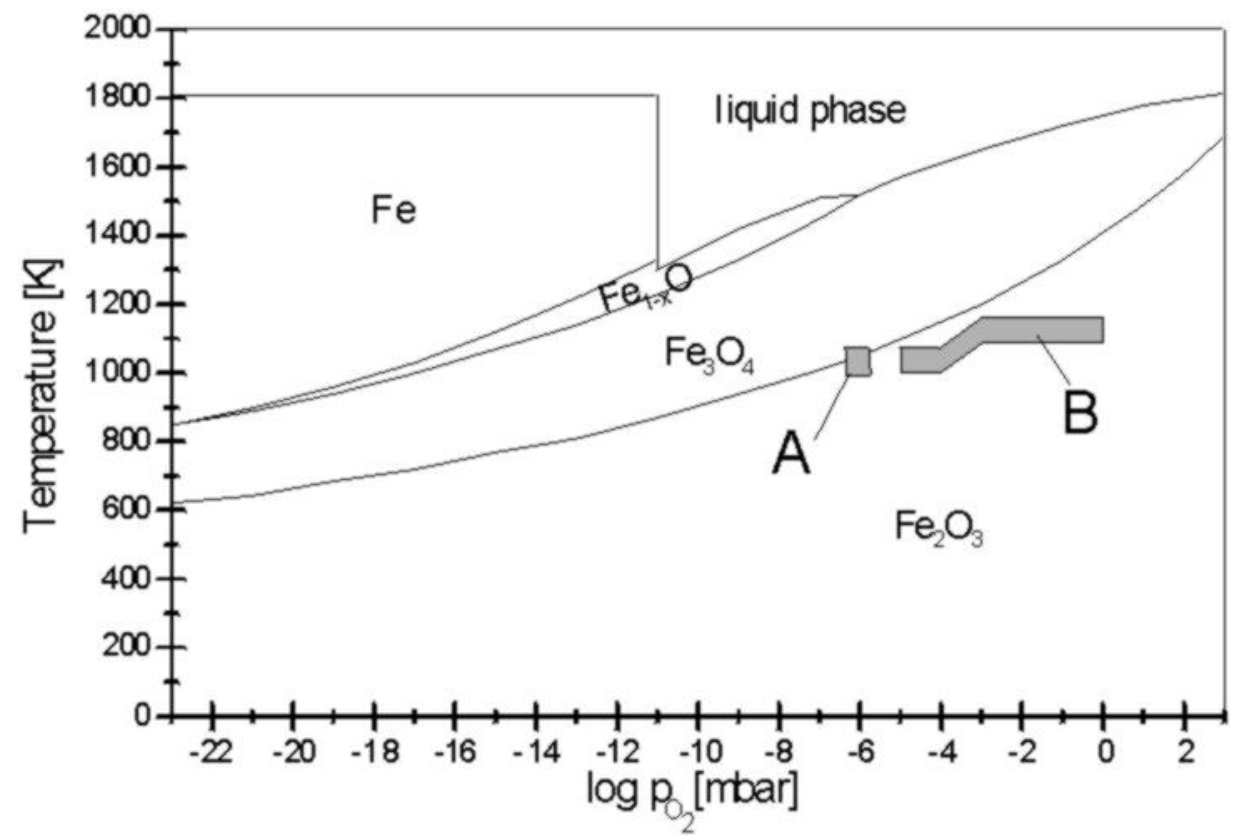

Fig. 5.1. $\mathrm{p}\left(\mathrm{O}_{2}\right)-\mathrm{T}$ phase diagram of $\mathrm{Fe}$ $\mathrm{O}_{2}$ system. Ranges where $\mathrm{Fe}_{3} \mathrm{O}_{4}(\mathrm{~A})$ and $\mathrm{Fe}_{2} \mathrm{O}_{3}$ (B) films were grown on $\mathrm{Pt}(111)$ are indicated. After [225].

Figure 5.2 presents perspective side and top views of the three Fe-oxide crystal structures studied in this work. They expose surface planes as obtained by truncating the bulk structure. The side views show the hexagonal Fe and $\mathrm{O}$ (111) and (0001) planes stacked consecutively along the [111] and [0001] crystal directions, the top views show the unreconstructed (111) and (0001) surfaces terminated by topmost Fe layers. The two-dimensional surface unit cells are indicated.

$\mathrm{FeO}$ wüstite crystallizes in the $\mathrm{NaCl}$ structure containing four formula units in the cubic unit cell. The large $\mathrm{O}^{2-}$ anions form a close packed fcc sublattice with the small $\mathrm{Fe}^{2+}$ cations located in the interstitial sites. All Fe ions are octahedrally coordinated to O. Under thermal equilibrium this phase is stable only for temperatures above $843 \mathrm{~K}$, below this temperature it disproportionates into metallic $\mathrm{Fe}$ and $\mathrm{Fe}_{3} \mathrm{O}_{4}$ [224]. Therefore, this oxide must be prepared above $\mathrm{T}=843 \mathrm{~K}$ and quenched afterwards down to room temperature. Usually, wüstite exhibits a large deviation from stoichiometry, $\mathrm{Fe}_{1-\mathrm{x}} \mathrm{O}$, with $\mathrm{x}$ extending from $5 \%$ up to $15 \%$, depending on the $\mathrm{O}_{2}$ partial pressure and temperature [228]. The $\mathrm{Fe}$ deficiency is caused by an oxidation of $\mathrm{Fe}^{2+}$ ions to $\mathrm{Fe}^{3+}$. The cubic lattice constant increases with increasing $\mathrm{Fe}$ content, ranging from $4.28 \AA$ to $4.32 \AA$ for stoichiometric $\mathrm{FeO}$ prepared at high $\mathrm{O}_{2}$ pressures (40-50 kbar) [ 229]. At $T=843 \mathrm{~K}$, the lattice constant of $\mathrm{Fe}_{0.95} \mathrm{O}$ is $4.31 \AA$ [230]. In this oxide, the $\mathrm{O}$ and $\mathrm{Fe}$ (111) planes form ideal two-dimensional hexagonal lattices with an interatomic distance of $3.04 \AA$, which corresponds to the lattice constant of the hexagonal unit cell on the unreconstructed $\mathrm{FeO}(111)$ surface, indicated in Fig. 5.2. Along the [111] direction the Fe and O (111) planes form the cubic $\mathrm{ABC}$ stacking sequence with an interlayer distance of $1.25 \AA$. The Fe-O bond length is $2.16 \AA$.

$\mathrm{Fe}_{3} \mathrm{O}_{4}$ magnetite is a ferrimagnet and crystallizes in the inverse spinel structure [224, 230]. The $\mathrm{O}$ anions form a close-packed fcc sublattice with $\mathrm{Fe}^{2+}$ and $\mathrm{Fe}^{3+}$ cations located in the interstitial sites. One cation site is tetrahedrally coordinated to $\mathrm{O}$ and is occupied only by $\mathrm{Fe}^{3+}$ ions. The other site is octahedrally coordinated to $\mathrm{O}$ and is occupied by equal numbers of $\mathrm{Fe}^{2+}$ and $\mathrm{Fe}^{3+}$ ions. The cubic unit cell has a lattice constant of $8.396 \AA$ and contains eight formula units, which can be written as $\left(\mathrm{Fe}^{3+}\right)_{8}\left[\mathrm{Fe}^{3+} \mathrm{Fe}^{2+}\right]_{8} \mathrm{O}_{32}$. The parantheses () denote the tetrahedrally coordinated $\mathrm{Fe}^{3+}$ ions, often labeled A sites, and the brackets [ ] denote the octahedrally coordinated $\mathrm{Fe}^{2+}$ and $\mathrm{Fe}^{3+}$ ions or $\mathrm{B}$ sites. The high electrical conductivity of magnetite at room temperature is attributed to electron hopping processes 
between the $\mathrm{Fe}^{2+}$ and $\mathrm{Fe}^{3+}$ ions. At $T \approx 120 \mathrm{~K}$, the conductivity decreases by two orders of magnitude, which is attributed to freezing of these electrons (Verwey transition) [231]. In the band structure picture, the high electical conductivity of $\mathrm{Fe}_{3} \mathrm{O}_{4}$ is a consequence of the half-filled $3 \mathrm{~d}$ band formed by the Fe atoms located at the B sites, and the Verwey transition induces a band splitting with a banggap opening up at the Fermi level [232].

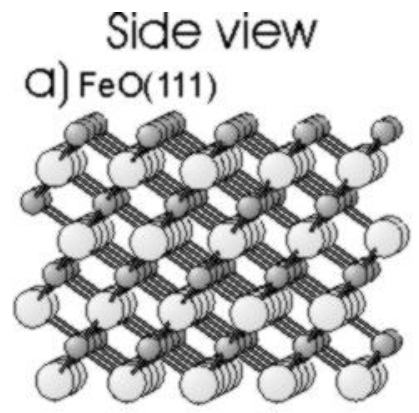

\section{Iron Layer structures}
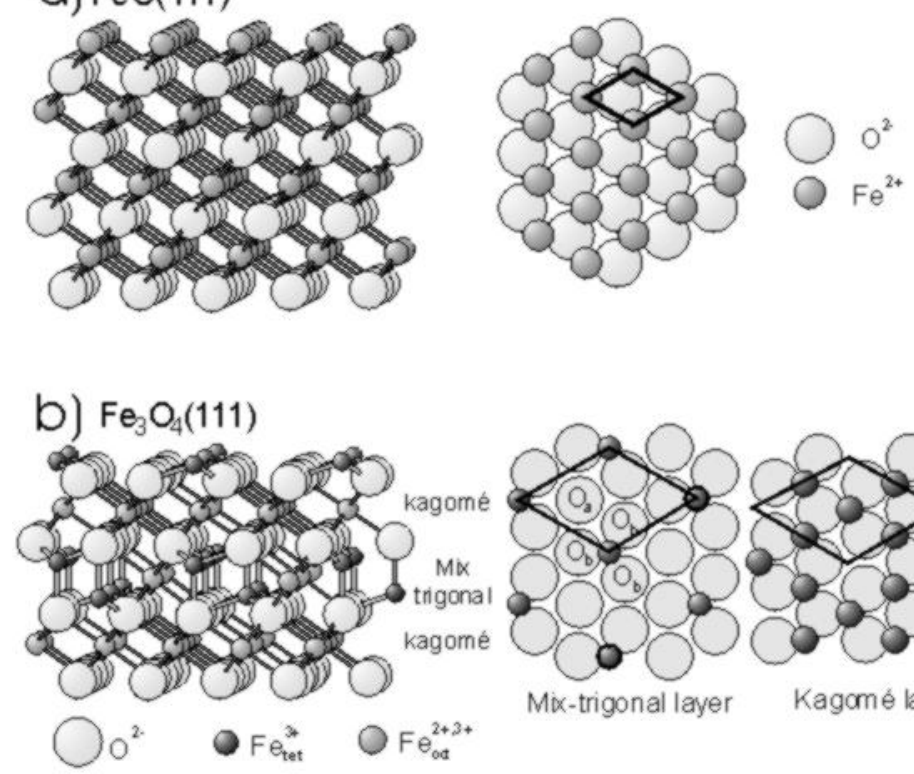

C) $\alpha-\mathrm{Fe}_{2} \mathrm{O}_{3}(0001)$

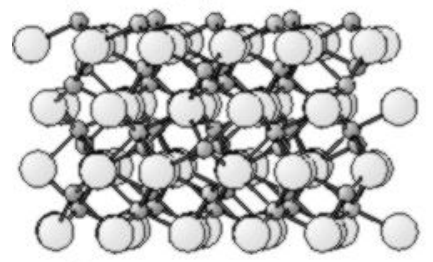

$\mathrm{O} \mathrm{Fe}^{3+}$

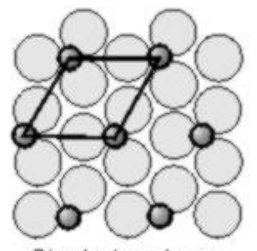

Single iron layer
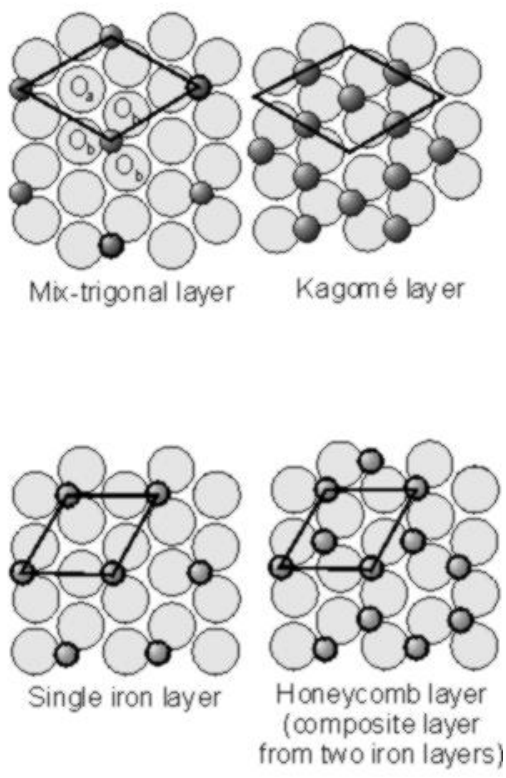

Fig. 5.2. Perspective side views of Feoxide crystal structures and top views cut parallel to close packed O layers. Bulk truncated (111) and (0001) surface structures terminated by outermost Fe planes are shown. Surface unit cells are indicated. Top views are drawn with full cation and anion sizes. In side views, ionic radii were reduced by factor of two. From [225].

Along the [111] axis of $\mathrm{Fe}_{3} \mathrm{O}_{4}$, the hexagonal $\mathrm{O}$ planes form a cubic $\mathrm{ABC}$ stacking sequence. Between the $\mathrm{O}$ (111) planes either one Kagomé or three hexagonal (mix-trigonal) Fe layers alternate. In the Kagomé layer, $3 / 4$ of the octahedrally coordinated $\mathrm{B}$ sites are occupied by $\mathrm{Fe}^{2+}$ and $\mathrm{Fe}^{3+}$ species. The unoccupied sites in these planes are $5.94 \AA$ apart forming an ordered hexagonal lattice. In the mixtrigonal layers, only $1 / 4$ of all sites are occupied within each subplane: The central plane contains octahedrally coordinated $\mathrm{Fe}^{3+}$ and $\mathrm{Fe}^{3+}$ species located on $\mathrm{B}$ sites, the other two planes tetrahedrally coordinated $\mathrm{Fe}^{3+}$ species located on A sites. The interatomic Fe-Fe distance within these planes is also $5.94 \AA$, which corresponds to the lattice constant of the two-dimensional surface unit cell of $\mathrm{Fe}_{3} \mathrm{O}_{4}(111)$ indicated in Fig. 5.2. There is a slight deviation of the $\mathrm{O}$ anion positions within the (111) planes from an ideal hexagonal arrangement, and the $\mathrm{p} 3 \mathrm{~m} 1$ point group symmetry of this surface is due to two types of $\mathrm{O}$ anions, which are denoted $\mathrm{O}_{\mathrm{a}}$ and $\mathrm{O}_{\mathrm{b}}$ in Fig. 5.2. The $\mathrm{O}_{\mathrm{a}}$ species are located $0.04 \AA$ closer to the topmost single octahedrally coordinated Fe layers than the $\mathrm{O}_{b}$ species. The average O-O interatomic distance within the (111) planes is $2.97 \AA$. If we consider weighted values for the $\mathrm{O}_{a}$ and $\mathrm{O}_{\mathrm{b}}$ sublayers, neighboring O (111) planes separated by the Kagomé layer are $2.37 \AA$ apart, O planes separated by the three mix-trigonal layers are $2.48 \AA$ apart. This leads to a distance of $4.85 \AA$ between 
every second $\mathrm{O}$ layer, which corresponds to the distance between equivalent (111) surface terminations of $\mathrm{Fe}_{3} \mathrm{O}_{4}$. The $\mathrm{Fe}$ subplanes of the mix-trigonal $\mathrm{Fe}$ layers are separated by $0.6 \AA$. The $\mathrm{Fe}-\mathrm{O}$ bond lenghts for tetrahedrally coordinated $\mathrm{Fe}$ is $1.88 \AA$, that for octahedrally $\mathrm{Fe}$ is $2.07 \AA$. The (111) surface plane is stable and frequently occurs on naturally grown crystals, which assume octahedral shapes [224].

$\gamma-\mathrm{Fe}_{2} \mathrm{O}_{3}$ maghemite is a metastable phase with a cubic crystal structure closely related to the $\mathrm{Fe}_{3} \mathrm{O}_{4}$ magnetite structure. When $\mathrm{Fe}_{3} \mathrm{O}_{4}$ is oxidized to $\gamma$ - $\mathrm{Fe}_{2} \mathrm{O}_{3}$, cation vacancies $(\otimes)$ are created at octahedral interstitials resulting in $\left(\mathrm{Fe}^{3+}\right)_{8}\left[\mathrm{Fe}^{3+}{ }_{5 / 3} \otimes_{1 / 3}\right]_{8} \mathrm{O}_{32}$. This is connected with a small decrease of the cubic lattice constant to values between 8.33 and $8.35 \AA$. Reasons for these different values may be that one often deals with solid solutions of $\mathrm{Fe}_{3} \mathrm{O}_{4}$ and $\gamma-\mathrm{Fe}_{2} \mathrm{O}_{3}$ rather than with a pure $\gamma-\mathrm{Fe}_{2} \mathrm{O}_{3}$ phase.

The phase diagram in Fig. 5.1 shows that $\alpha-\mathrm{Fe}_{2} \mathrm{O}_{3}$ hematite is the only Fe-oxide phase that is stable at room temperature in thermodynamic equilibrium with ambient $\mathrm{O}_{2}$ athmospheres. It crystallizes in the corundum structure with the hexagonal unit cell containing six formula units. The lattice constants are $a=5.035 \AA$ and $c=13.72 \AA[230]$. The $\mathrm{O}$ anions form an hcp sublattice with ABAB stacking. The $\mathrm{Fe}^{3+}$ species in the interstitials are arranged in distorted octahedra and form two sublayers. The interatomic distance within these Fe layers is $5.03 \AA$, which corresponds to the lattice constant of the two-dimensional unit cell of an unreconstructed (0001) surface, as indicated in the top view of Fig. 5.2. The $\mathrm{O}$ anion positions within the (111) planes also deviate slightly from an ideal hexagonal arrangement. The average O-O interatomic distance is $2.91 \AA$. Due to the distortion of the octahedra there are two different Fe-O bond lenghts, $1.96 \AA$ and $2.09 \AA$. The two Fe subplanes in between the O planes are separated by $0.6 \AA$. The distance between two O planes is $2.29 \AA$, which corresponds to the distance between equivalent (0001) surface terminations.

The quasi-hexagonal surfaces of the three Fe-oxide phases in Fig. 5.2 form three different unit cells, provided no reconstructions occur. They have lattice constants of $3.04 \AA(\mathrm{FeO}), 5.94 \AA\left(\mathrm{Fe}_{3} \mathrm{O}_{4}\right)$ and $5.03 \AA\left(\alpha-\mathrm{Fe}_{2} \mathrm{O}_{3}\right)$. The $\alpha-\mathrm{Fe}_{2} \mathrm{O}_{3}(0001)$ unit cell is rotated by $30^{\circ}$ with respect to the others. Therefore the LEED patterns of these surfaces can be used to identify the different Fe-oxide phases.

\subsection{Identification of iron-oxide phases}

\subsubsection{LEED patterns}

Figure 5.3 shows the LEED patterns of the clean Pt(111) substrate surface and three different Feoxide films grown onto the $\operatorname{Pt}(111)$ surface, together with top views of the corresponding surface structures. The surface terminations are different from those in Fig. 5.2 and represent the most stable terminations, as deduced from the investigations presented in this section. The $(1 \times 1)$ unit cells in realspace and reciprocal-space are indicated. The hexagonal unit cell of the $\operatorname{Pt}(111)$ surface has a lattice constant of $2.77 \AA$, which corresponds to the positions of the first-order diffraction spots visible in its LEED pattern. The LEED pattern in (b) is due to the first Fe-oxide layer, which consists of a hexagonal $\mathrm{Fe}-\mathrm{O}$ bilayer forming an $\mathrm{FeO}(111)$ like structure. Six main diffraction spots are surrounded by rosettes of satellite spots. The main spot positions correspond to a periodicity of about $3.1 \AA$, slightly larger than expected for an unreconstructed $\mathrm{FeO}(111)$ surface $(3.04 \AA)$. The satellite spots are caused by the $12 \%$ lattice mismatch with respect to the Pt substrate, as discussed in Section 5.3. The $\mathrm{FeO}(111)$ monolayer film forms an unreconstructed surface terminated by a close-packed $\mathrm{O}$ layer. This is known from X-ray absorption spectroscopy [233], atomic resolution STM images in conjunction with theoretical image simulations [163], and from photoelectron diffraction experiments [164].

Figure 5.3c displays the LEED pattern of an $\mathrm{Fe}_{3} \mathrm{O}_{4}(111)$ film, which is at least $100 \AA$ thick. The satellite spots of the first $\mathrm{FeO}(111)$ layers have disappeared and the first-order spot positions correspond to a periodicity of about $6 \AA$, as expected for an unreconstructed $\mathrm{Fe}_{3} \mathrm{O}_{4}(111)$ surface 
structure (5.92 $\AA$ ). The dynamical LEED structure analysis and the atomic resolution STM images presented in Section 5.6 reveal a surface structure exposing $1 / 4$ monolayer of tetrahedrally coordinated $\mathrm{Fe}$ atoms in the topmost layer.Figure 5.3d shows the LEED pattern obtained after oxidizing the $\mathrm{Fe}_{3} \mathrm{O}_{4}(111)$ film in $10^{-1} \mathrm{mbar}_{2}$. The first-order spot positions correspond to a $5 \AA$ periodicity along a direction rotated by $30^{\circ}$ with respect to the atom rows on the $\mathrm{Fe}_{3} \mathrm{O}_{4}(111)$ surface. This corresponds to an $\alpha-\mathrm{Fe}_{2} \mathrm{O}_{3}(0001)$ film at least $100 \AA$ thick, forming an unreconstructed $(1 \times 1)$ surface structure. As presented in Section 5.7, different surface terminations are formed on these films, depending on the preparation conditions. The LEED patterns in Fig. 5.3 also reflect the epitaxial relationships between the oxide overlayers and the Pt substrate. The marked unit cells correspond to the hexagonal unit cells formed on the $\mathrm{O}$ (111) planes of the three oxide films, which ideally have lattice constants of $3.04 \AA$ (FeO), $2.97 \AA\left(\mathrm{Fe}_{3} \mathrm{O}_{4}\right)$, and $2.90 \AA\left(\alpha-\mathrm{Fe}_{2} \mathrm{O}_{3}\right)$. The unit cells of $\mathrm{Fe}_{3} \mathrm{O}_{4}$ and $\alpha-\mathrm{Fe}_{2} \mathrm{O}_{3}$ are aligned with the $\mathrm{Pt}(111)$ LEED spots, which means that the atomic rows in the $\mathrm{O}$ sublattices are parallel to the atomic rows of the $\mathrm{Pt}(111)$ substrate. The spots of the FeO film are slightly elongated (hardly visible in Fig. $5.3 \mathrm{~b}$ ), due to a small rotation of the O lattice with respect to the Pt substrate see Section 5.3.
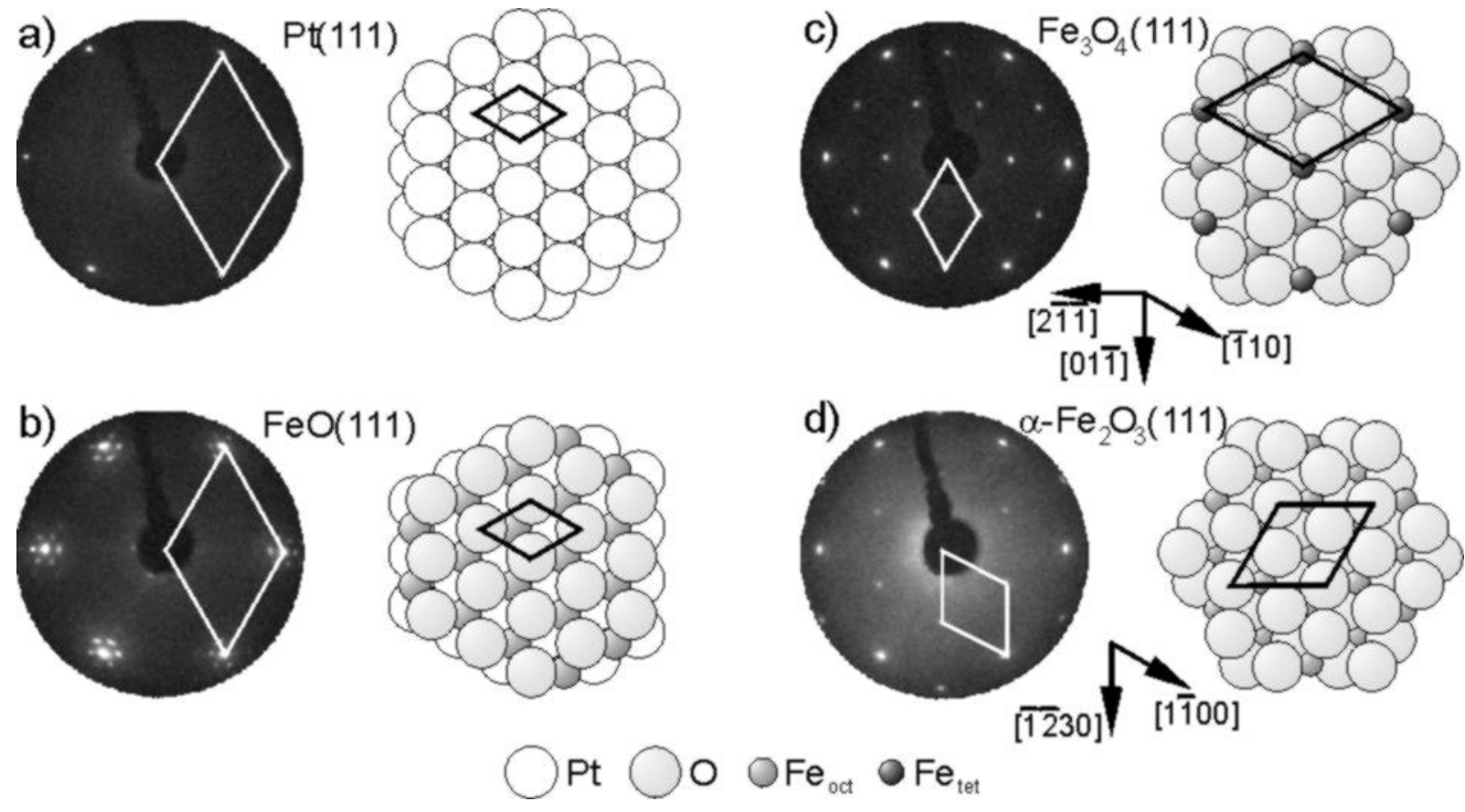

Fig. 5.3. LEED patterns at $E=60 \mathrm{eV}$ and top views of corresponding surface structures of $\operatorname{Pt}(111)$ substrate (a), and of different Fe-oxide films grown onto $\mathrm{Pt}(111)$ (b) to (d). Unit cells in real and reciprocal space and crystallographic directions in cubic (a-c) and hexagonal crystal structures (d) are indicated. Epitaxial relationships between oxide films and substrate surface lattice is reflected in this figure. After [234].

\subsubsection{AES and XPS measurements}

Figure 5.4 shows AES spectra of three Fe-oxide films. They were scaled, so that the Fe signals at $651 \mathrm{eV}$ kinetic energy had the same amplitude. No contamination signals are seen, neither here nor in the XPS survey scans (not shown here). For the FeO monolayer film, the Pt signal at $237 \mathrm{eV}$ has about the same amplitude as the Fe signal at $651 \mathrm{eV}$, which corresponds to at least $1 \mathrm{ML}$ thickness, in agreement with combined AES and ion scattering spectroscopy measurements [160]. As expected, the $\alpha-\mathrm{Fe}_{2} \mathrm{O}_{3}$ film has a higher $\mathrm{O} / \mathrm{Fe}$ intensity ratio than the $\mathrm{Fe}_{3} \mathrm{O}_{4}$ film. 
Figure 5.5 shows XPS spectra measured of metallic Fe and the three Fe-oxide films in the range covering the $\mathrm{O}_{\mathrm{KLL}}$ Auger peak and the Fe $2 p$ photoemission peaks. The Fe $2 p$ core levels are split into $2 p_{1 / 2}$ and $2 p_{3 / 2}$ components, due to the spin-orbit coupling. The Fe2 $p_{3 / 2}$ core-level of elemental Fe appears at $706.3 \mathrm{eV}$, that of of the $\alpha-\mathrm{Fe}_{2} \mathrm{O}_{3}$ multilayer film and of the $\mathrm{FeO}$ monolayer film at $711.3 \mathrm{eV}$ and $710 \mathrm{eV}$ binding energy, respectively. These values are characteristic for the $\mathrm{Fe}^{3+}$ and $\mathrm{Fe}^{2+}$ species and agree well with literature data $[29,197]$. On $\alpha-\mathrm{Fe}_{2} \mathrm{O}_{3}$ the shake-up satellite line characteristic for

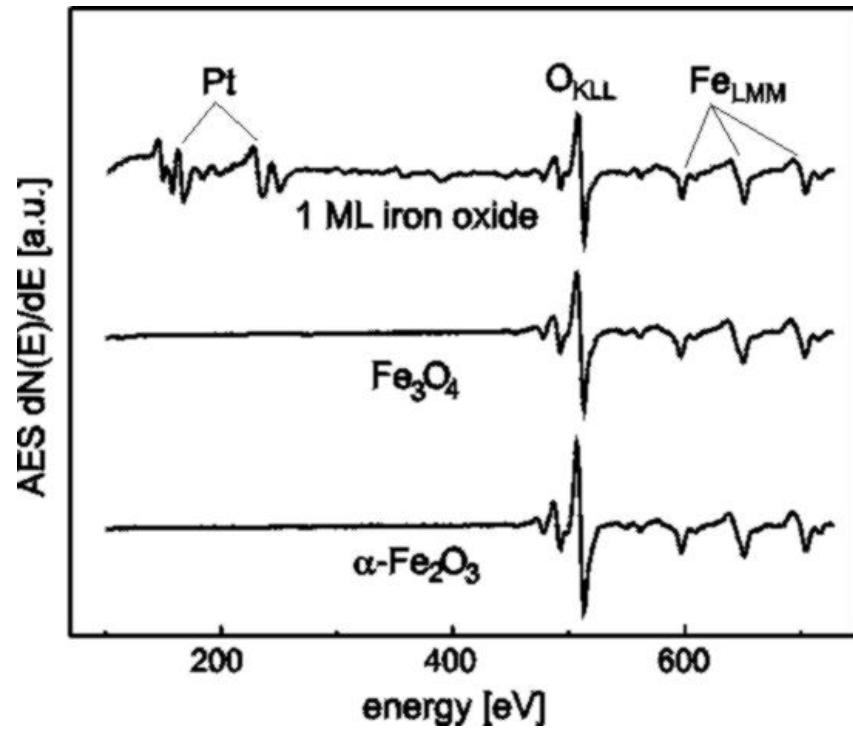

Fig. 5.4: AES spectra of epitaxial Fe-oxide films grown onto $\mathrm{Pt}(111)$. $\mathrm{FeO}(111)$ film is $1-2 \mathrm{ML}$, $\mathrm{Fe}_{3} \mathrm{O}_{4}(111)$ and $\alpha-\mathrm{Fe}_{2} \mathrm{O}_{3}(0001)$ films are at least 100 Å thick. From [235].

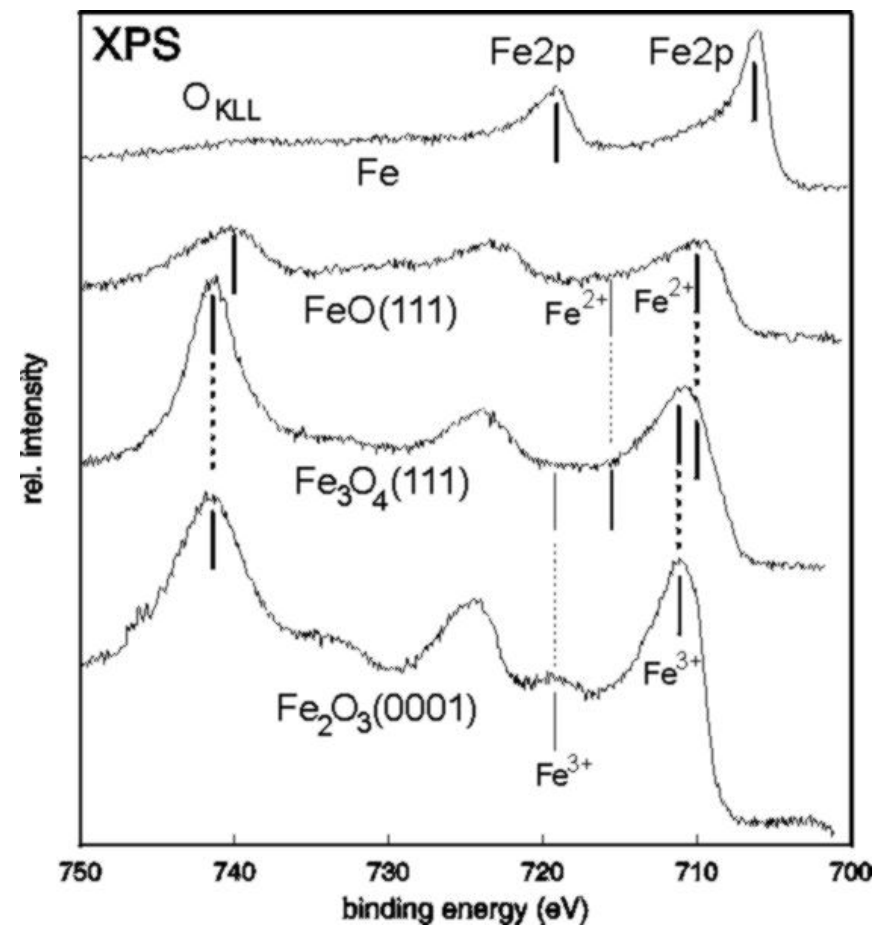

Fig. 5.5. XPS spectra of evaporated elemental Fe film (a) and of epitaxial Fe-oxide films grown onto Pt(111). 1-2 ML thick FeO(111) film (b), and at least $100 \AA$ thick $\mathrm{Fe}_{3} \mathrm{O}_{4}(111)$ (c) and $\alpha$ $\mathrm{Fe}_{2} \mathrm{O}_{3}(0001)$ films (d). After [278].

the $\mathrm{Fe}^{3+}$ species [29] at $719.2 \mathrm{eV}$ binding energy is clearly visible. The $\mathrm{Fe} 2 \mathrm{p}_{3 / 2}$ signal in the $\mathrm{FeO}$ monolayer spectrum is asymmetrically broadened, when compared to the $\alpha-\mathrm{Fe}_{2} \mathrm{O}_{3}$ spectrum, which can be attributed to a satellite excitation of the $\mathrm{Fe}^{2+}$ species [197]. Magnetite $\mathrm{Fe}_{3} \mathrm{O}_{4}$ contains both $\mathrm{Fe}^{3+}$ and $\mathrm{Fe}^{2+}$ species, which also causes a broader $\mathrm{Fe} 2 \mathrm{p}$ signals than that of the $\alpha-\mathrm{Fe}_{2} \mathrm{O}_{3}$ multilayer. The binding energy of the O1s peak (not shown here, see [233]) is independent of the oxide phase at 530.1 $\mathrm{eV}$, in agreement with literature $[29,197]$. In contrast, the $\mathrm{O}_{\mathrm{KLL}}$ emission of the thin FeO film is shifted by about $1.5 \mathrm{eV}$ towards higher kinetic energy, when compared to $\mathrm{Fe}_{3} \mathrm{O}_{4}$ and $\mathrm{Fe}_{2} \mathrm{O}_{3}$. It is unlikely that this is due to the coordination of $\mathrm{O}$ to $\mathrm{Fe}^{2+}$, since emission at this energy should then also appear in the spectrum of $\mathrm{Fe}_{3} \mathrm{O}_{4}$, which is not observed. It is more likely that it is due to the complexity of the Auger process, which involves three levels, two of which are L-shell levels that corresponds to the $\mathrm{O}$ valence electrons, depends on many-body effects and has a two-hole final-state.

In summary, the AES spectra in Fig. 5.4 and XPS spectra in Fig. 5.5 of the three Fe-oxide films agree well with spectra measured on corresponding polycrystalline or single-crystal bulk samples. 


\subsubsection{UPS valence-band spectra}

The valence and conduction bands of the Fe-oxides are formed by Fe3d states that are hybridized with $\mathrm{O} 2 \mathrm{p}$ states [182, 236-238]. This hybridization and the complexity of electron correlation effects make the interpretation of the valence-band photoemission spectra difficult. Resonant photoemission allows us to separate the Fe3d derived states in the spectra. Excitations of the type $3 \mathrm{p}^{6} 3 \mathrm{~d}^{\mathrm{n}} \rightarrow$ $\left[3 p^{5} 3 d^{n+1}\right]^{*}$ (the asterisk denotes an excited state) dominate at the Fe3p threshold. These excitations decay to a $3 \mathrm{p}^{6} 3 \mathrm{~d}^{\mathrm{n}-1}+\mathrm{e}^{-}$state thereby resonantly enhancing the direct photoemission process $3 \mathrm{p}^{6} 3 \mathrm{~d}^{\mathrm{n}} \rightarrow$ $3 \mathrm{p}^{6} 3 \mathrm{~d}^{\mathrm{n}-1}+\mathrm{e}^{-}$. By taking the difference between valence-band energy distribution curves (EDC's) measured just above and below the $\mathrm{Fe} 3 \mathrm{p} \rightarrow 3 \mathrm{~d}$ resonance energy, the nonresonating $\mathrm{O} 2 \mathrm{p}$ contributions are eliminated and the distribution of $\mathrm{Fe} 3 \mathrm{~d}$ derived final states is obtained. This was done for the first time by Fujimori et al. on in-situ scraped surfaces of $\mathrm{Fe}_{\mathrm{x}} \mathrm{O}$ and $\alpha-\mathrm{Fe}_{2} \mathrm{O}_{3}$ single-crystals [239, 240], later by $\mathrm{Lad}$ et al. on cleaved $\mathrm{FeO}, \mathrm{Fe}_{3} \mathrm{O}_{4}$ and $\alpha-\mathrm{Fe}_{2} \mathrm{O}_{3}$ single-crystals [241]. Furthermore, constantinitial-state (CIS) spectra measured across the $\mathrm{Fe} 3 \mathrm{p}$ excitation threshold can be used to distinguish the $3 \mathrm{~d}^{\mathrm{n}-1}$ from the $3 \mathrm{~d}^{\mathrm{n}} \mathrm{L}$ photoemission final states, i.e., one can identify strongly hybridized Fe-O states in the valence-band ( $\mathrm{L}$ is a hole state in an O ligand) [239, 240, 242-244]. These studies revealed that, in all Fe-oxides, $3 \mathrm{~d}^{\mathrm{n}} \mathrm{L}$ final states are located predominantly between the Fermi level and $9 \mathrm{eV}$ binding energy, whereas $3 \mathrm{~d}^{\mathrm{n}-1}$ final states are located between 9 and $16 \mathrm{eV}$ binding energy. These observations and the large spectral valence-band width of $16 \mathrm{eV}$ can also be modeled by configuration-interaction cluster calculations on $\left[\mathrm{FeO}_{6}\right]^{10-}$ and $\left[\mathrm{FeO}_{6}\right]^{9-}$ clusters representing $\mathrm{FeO}$ and $\alpha-\mathrm{Fe}_{2} \mathrm{O}_{3}$, respectively [240].

Figure 5.6 shows angle integrated valence-band EDC's for a $\mathrm{Fe}_{3} \mathrm{O}_{4}(111)$ multilayer film taken with $h v=120 \mathrm{eV}$ photon energy (a), and for an $\alpha-\mathrm{Fe}_{2} \mathrm{O}_{3}(0001)$ multilayer film taken with $h v=100 \mathrm{eV}$ photon energy (b). In both spectra, the main intensity arises from Fe3d states, due to their higher photoemission cross-sections at these photon energies $[245,246]$. In the $\mathrm{Fe}_{3} \mathrm{O}_{4}$ spectrum, a feature at $1.2 \mathrm{eV}$ binding energy can be seen, which is assigned to a $3 \mathrm{~d}^{6} \mathrm{~L}$ final state of $\mathrm{Fe}^{2+}$ species. This feature is not present in the $\alpha-\mathrm{Fe}_{2} \mathrm{O}_{3}$ spectrum, which also indicates a pure $\alpha-\mathrm{Fe}_{2} \mathrm{O}_{3}$ phase in the surface region as these spectra are very surface sensitive. $3 \mathrm{~d}^{4}$ final-state emission can be seen between 10 and $18 \mathrm{eV}$ binding energy in the $\alpha-\mathrm{Fe}_{2} \mathrm{O}_{3}$ film, whereas in the $\mathrm{Fe}_{3} \mathrm{O}_{4}$ film containing $\mathrm{Fe}^{2+}$ and $\mathrm{Fe}^{3+}$ species these features are less well resolved, due to overlap of $3 \mathrm{~d}^{4}$ and $3 \mathrm{~d}^{5}$ final states. In addition to the LEED patterns observed on these two films, the photoemission measurements also indicate that single phased $\mathrm{Fe}_{3} \mathrm{O}_{4}$ magnetite and $\alpha-\mathrm{Fe}_{2} \mathrm{O}_{3}$ hematite films have formed on the $\mathrm{Pt}(111)$ substrate.

Figure 5.7 presents angle-integrated valence-band EDC's of the $\mathrm{FeO}(111)$ monolayer. The spectra are taken just above $(\mathrm{hv}=60 \mathrm{eV}$, Fig. 5.7a) and below $(\mathrm{hv}=50 \mathrm{eV}$, Fig. 5.7b) the Fe3p threshold. Corresponding spectra from a cleaved $\mathrm{Fe}_{\mathrm{x}} \mathrm{O}(100)$ single-crystal surface measured by Lad and Henrich [241] are shown for comparison. The difference spectra between spectra (a) and (b) reflecting the Fe3d derived final states are shown at the bottom. The $3 \mathrm{~d}$-derived emission extend down to $20 \mathrm{eV}$ below the Fermi level, which is in general agreement with the results of Lad et al. for a cleaved $\mathrm{Fe}_{\mathrm{x}} \mathrm{O}(100)$ single-crystal surface. However, the shape of the spectra differs significantly, indicating a different electronic structure for the $\mathrm{FeO}(111)$ film. This might be due to Fe-Pt interaction at the substrateoverlayer interface, leading to Fe3d final-state features coupled with the Pt electronic states, or to the formation of a completely different band structure, since the $\mathrm{FeO}(111)$ film represents a twodimensional system.

All features observed in the valence-band spectra of the $\mathrm{Fe}_{3} \mathrm{O}_{4}$ and $\alpha-\mathrm{Fe}_{2} \mathrm{O}_{3}$ multilayer films agree well with data from polycrystalline and single-crystal bulk samples, indicating the equivalence of these multilayer films to the corresponding bulk oxides. This is further confirmed by angle-resolved photoemission measurements using synchrotron radiation performed on the $\mathrm{Fe}_{3} \mathrm{O}_{4}(111)$ multilayer films [247]. The observed band dispersion along the $\Gamma$-L symmetry line of the first Brillouin zone 
agrees reasonably well with band-structure calculations for bulk $\mathrm{Fe}_{3} \mathrm{O}_{4}$. The $\mathrm{FeO}$ monolayer film represents a two-dimensional system and has a different electronic structure than bulk FeO.

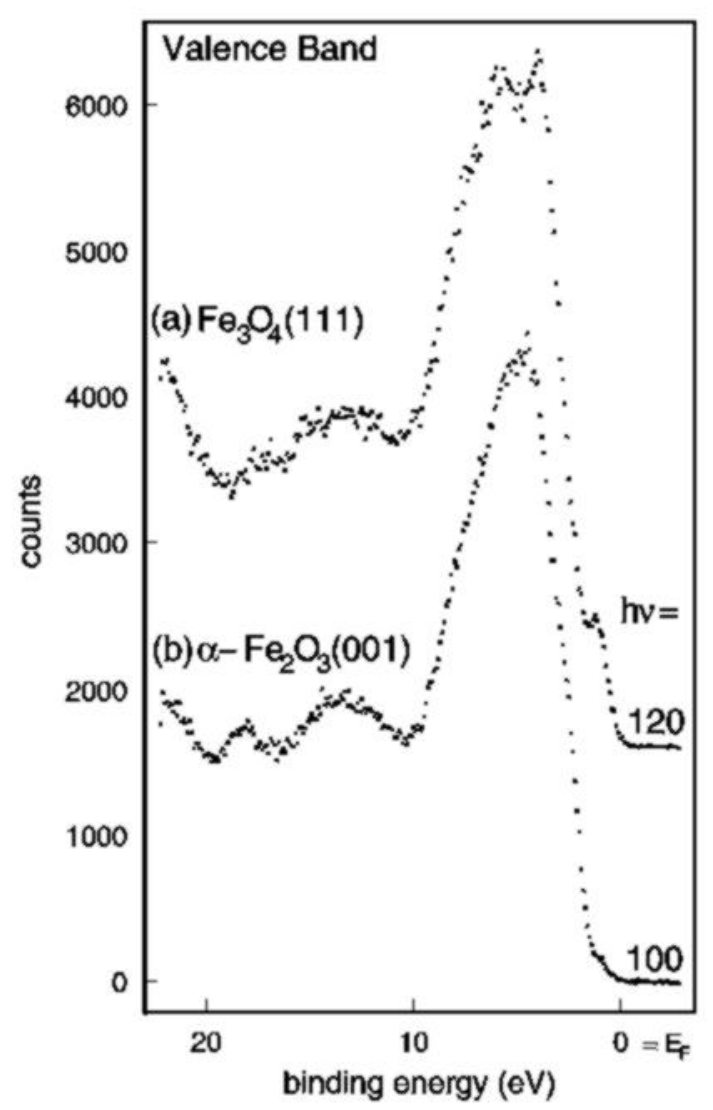

Fig. 5.6. (Left) Valence-band photoemission spectra of (a) $\mathrm{Fe}_{3} \mathrm{O}_{4}(111)$ and (b) $\alpha-\mathrm{Fe}_{2} \mathrm{O}_{3}(0001)$ multilayer films [233].

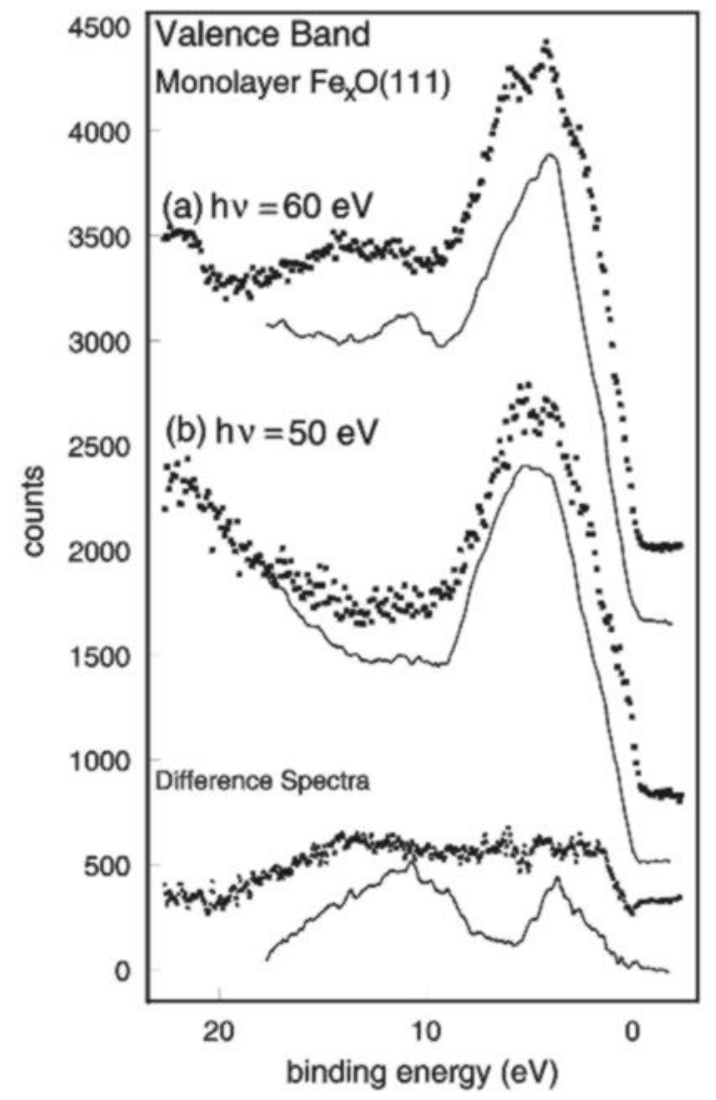

Fig. 5.7. (Right) Valence-band photoemission spectra of a $\mathrm{FeO}(111)$ monolayer film, taken at photon energies above (a) and below $\mathrm{Fe} 3 \mathrm{p}$ absorption edge (b). For comparison, corresponding valence-band data of cleaved $\mathrm{FeO}(100)$ single-crystal surfaces from Lad and Henrich [241] are shown. From [233].

\subsubsection{XAS measurements}

Figure 5.8 shows the $\mathrm{O}$ K-edge absorption spectra of the $\mathrm{FeO}(111)$ monolayer (a), the $\mathrm{Fe}_{3} \mathrm{O}_{4}(111)$ multilayer (b) and the $\alpha-\mathrm{Fe}_{2} \mathrm{O}_{3}(001)$ multilayer film (c) for two angles $\Theta_{e}$ between the $\boldsymbol{E}$-vector of the light and the surface normal, $\Theta_{e}=20^{\circ}$ and $90^{\circ}$. The intensities of the XAS spectra were normalized to the edge jump at $\approx 555 \mathrm{eV}$ photon energy. The data are in good agreement with spectra from natural $\alpha$ $\mathrm{Fe}_{2} \mathrm{O}_{3}$ and $\mathrm{Fe}_{3} \mathrm{O}_{4}$ mineral samples [248] and with electron-energy-loss spectroscopy (EELS) data for $\mathrm{Fe}_{\mathrm{x}} \mathrm{O}, \mathrm{Fe}_{2} \mathrm{O}_{3}$ and $\mathrm{Fe}_{3} \mathrm{O}_{4}$ thin films [249].

In a first approximation, the O K-edge XAS spectra can be interpreted as an image of the O pprojected unoccupied density of states, that is the weight of the $\mathrm{O} 2 \mathrm{p}$ states in the conduction band [250]. In a pure ionic model, the oxide anion would have the configuration $O 1 s^{2} 2 s^{2} 2 p^{6}$ and the $1 s \rightarrow 2 p$ 


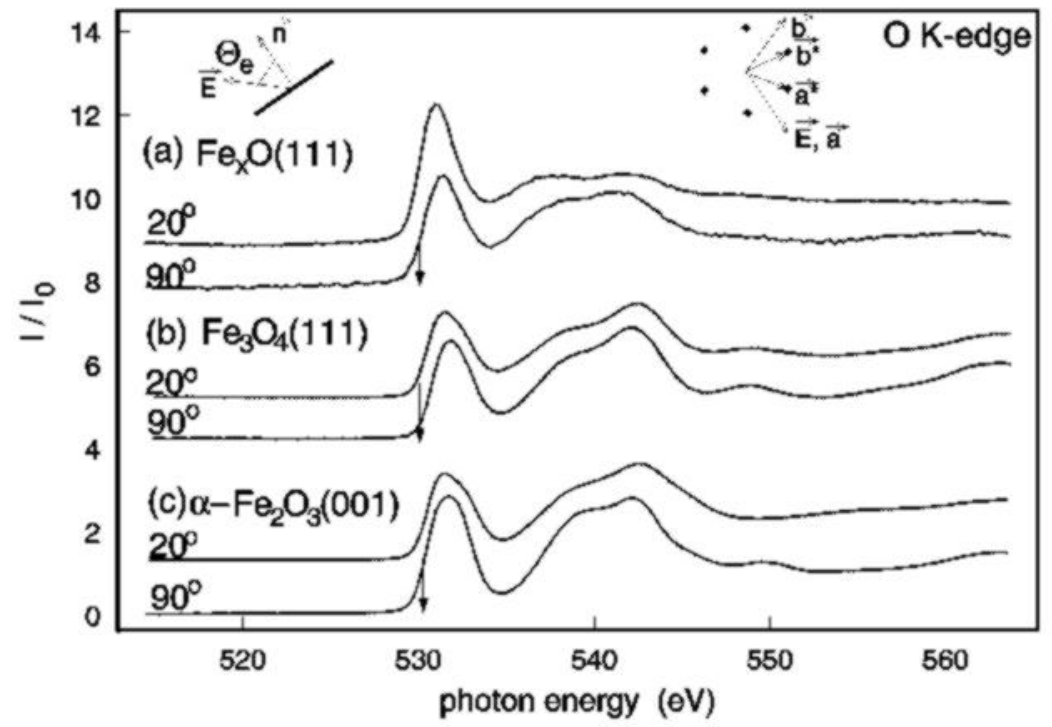

Fig.5.8. O K-edge X-ray

absorption spectra of ordered Fe-

oxide films on Pt(111): (a)

$\mathrm{FeO}(111)$ monolayer film, (b)

$\mathrm{Fe}_{3} \mathrm{O}_{4}(111)$ and (c) $\alpha-\mathrm{Fe}_{2} \mathrm{O}_{3}(111)$

multilayer films. All spectra are shown for two angles between $\boldsymbol{E}$ vector of light and surface normal, $\Theta_{e}=20^{\circ}$ and $90^{\circ}$. Arrows indicate

Fermi level positions. Insert:

Schematic (1x1) LEED pattern with surface lattice vectors in real space ( $\boldsymbol{a}$ and $\boldsymbol{b}$ ) and in reciprocal space $\left(\boldsymbol{a}^{*}\right.$ and $\left.\boldsymbol{b}^{*}\right)$ [233].

channel would be closed in XAS. Covalency reduces the number of filled states with O2p character, so that the strength of the O1s signal at the threshold is related to the degree of covalency. All spectra exhibit a sharp white line at about $533 \mathrm{eV}$ and a broader structure with two peaks at about $542 \mathrm{eV}$. The white line is assigned to the $\mathrm{O} 2 \mathrm{p}-\mathrm{Fe} 3 \mathrm{~d}$ band and the broader structure $5-20 \mathrm{eV}$ above the threshold to the O2p-Fe4sp band. This assignment agrees with molecular-orbital calculations [248, 251-253]. The broad Fe4sp band at $542 \mathrm{eV}$ also indicates a significant covalent bonding between Fe4sp states and $\mathrm{O} 2 \mathrm{p}$ states. It consists always of two peaks separated by $\approx 3.8 \mathrm{eV}$ for the multilayers and by $\approx 4.5 \mathrm{eV}$ for the monolayer film. In accordance to the $\mathrm{O}$ K-edges of the 3d-transition metal-oxides [248] this structure can be related to the symmetry set up by nearest $\mathrm{O}$ neighbors. The increased splitting of $\approx 4.5$ $\mathrm{eV}$ for the $\mathrm{FeO}(111)$ monolayer indicates a different local surrounding of the $\mathrm{Fe}^{2+}$ cations in the monolayer film. One also observes a different intensity ratio between the first sharp Fe $3 \mathrm{~d}$ unoccupied band at $\approx 533 \mathrm{eV}$ and the broader Fe4sp band at $\approx 542 \mathrm{eV}$. For the $\mathrm{FeO}(111)$ monolayer, this intensity ratio is much larger than for the multilayer spectra of $\mathrm{Fe}_{2} \mathrm{O}_{3}$ and $\mathrm{Fe}_{3} \mathrm{O}_{4}$. The reason for this may be the increase in the number of unoccupied $3 \mathrm{~d}$ states available for mixing with $\mathrm{O} 2 \mathrm{p}$ states (hybridization) within the monolayer, which can be related to either the different local surroundings of the Fe cations within the mono- and multilayers or to the coupling of occupied Fe3d states with unoccupied Pt5d states at the substrate-overlayer-interface. In analogy to the photoemission spectra, the XAS measurements show that the electronic structure of the $\mathrm{FeO}(111)$ monolayer film is different from that of $\mathrm{FeO}$ bulk crystals.

To a first approximation, the electronic structure of metal-oxides can be described by molecularorbital theory. In the field of the surrounding ligands, the degeneracy of the five Fe3d states is lifted, because of their different orbital overlaps with the $O$ ligands [254]. In an octahedral complex, the metal $e_{g}\left(d_{z^{2}}, d_{x^{2}-y^{2}}\right)$ states are pushed up in energy with respect to the $t_{2 g}\left(d_{x y}, d_{x z}, d_{y z}\right)$ states. This ligand-field splitting is reversed in tetrahedral complexes. In addition, one has to consider the exchange repulsion between the $d$ electrons, which splits the $t_{2 g}$ and $e_{g}$ bands into two bands, each resulting in the corresponding spin-up and spin-down bands. Tossell et al. calculated the molecularorbital energies of octahedrally coordinated $\mathrm{Fe}^{2+}$ and $\mathrm{Fe}^{3+}$ ions in $\left[\mathrm{FeO}_{6}\right]^{10-}$ and $\left[\mathrm{FeO}_{6}\right]^{9-}$ clusters representing $\mathrm{FeO}$ and $\alpha-\mathrm{Fe}_{2} \mathrm{O}_{3}$, respectively [255]. For both oxides, the exchange splitting is larger than the ligand-field splitting, leading to high spin complexes. The absolute values are given in the energy diagrams in Fig. $5.9 \mathrm{~b}$ and c. A trigonal coordination for the $\mathrm{Fe}^{2+}$ species was assumed in the $\mathrm{FeO}(111)$ monolayer film in Fig. 5.9a, which leads to a crystal-field splitting like that in a tetrahedral complex. 
(a) Monolayer $\mathrm{Fe}_{x} \mathrm{O}$ (111)

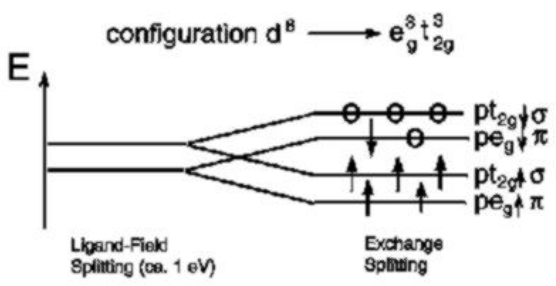

(b) $[\mathrm{FeO}]^{10-}$ Cluster for Wustite FeO

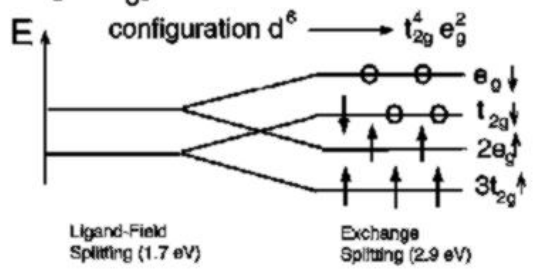

(c) $[\mathrm{FeQ}]^{9-}$ Cluster for Hematite $\mathrm{Fe}_{2} \mathrm{O}_{3}$

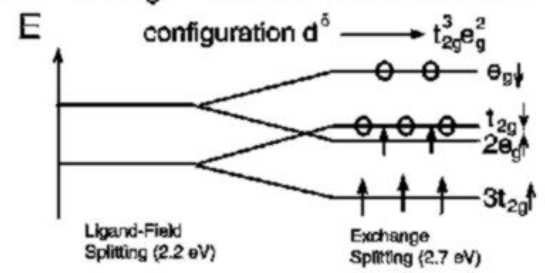

Fig. 5.9. Molecular-orbital energy diagrams (a) for trigonally coordinated $\mathrm{Fe}^{2+}$ species in $\mathrm{FeO}(111)$ monolayer film, (b) for $\left[\mathrm{FeO}_{6}\right]^{10-}$ cluster representing octahedrally coordinated $\mathrm{Fe}^{2+}$ species in $\mathrm{FeO}$, and (c) for $\left[\mathrm{FeO}_{6}\right]^{9-}$ cluster representing octahedrally coordinated $\mathrm{Fe}^{3+}$ species in $\alpha-\mathrm{Fe}_{2} \mathrm{O}_{3}$

[255]. Indicated ligand-field and exchange splittings were determined in [255] by $\mathrm{MO}$ calculations. Ligand-field splitting of $1 \mathrm{eV}$ for monolayer film was obtained by fitting experimantal data with two Gaussian/Lorenzian curves [233].

Based on these MO diagrams, the broadening of the white lines are identified as excitation into the $\mathrm{t}_{2 \mathrm{~g} \downarrow}$ and $\mathrm{e}_{\mathrm{g} \downarrow}$ symmetry bands separated by the ligand-field splitting, as shown in Fig. 5.9. For the $\mathrm{Fe}_{3} \mathrm{O}_{4}$ and $\alpha-\mathrm{Fe}_{2} \mathrm{O}_{3}$ multilayer films, we fitted the white lines with two combined Gaussian/Lorenzian functions separated by a ligand-field splitting of 1.3 and $1.5 \mathrm{eV}$, respectively. This is somewhat smaller than the values from the MO calculations ( $2.2 \mathrm{eV}$ "crystal-field splitting" for the $\left[\mathrm{FeO}_{6}\right]^{9-}$ cluster), but it is in reasonable agreement with recently published data for natural minerals [248] $(0.9 \mathrm{eV} / 1.3 \mathrm{eV}$ for $\left.\mathrm{Fe}_{3} \mathrm{O}_{4} / \alpha-\mathrm{Fe}_{2} \mathrm{O}_{3}\right)$. The splitting agrees also with optical data, which reveal a ligand-field splitting of 1.6 $\mathrm{eV}$ for the octahedrally hydrated $\mathrm{Fe}^{3+}$ ion [256]. From the fit, we obtain a ligand-field splitting of $1^{\circ} \mathrm{eV}$ for the trigonally coordinated $\mathrm{Fe}^{2+}$ species in the $\mathrm{FeO}$ monolayer film.

In summary, the XAS measurements also demonstrate that the $\mathrm{Fe}_{3} \mathrm{O}_{4}$ and $\alpha-\mathrm{Fe}_{2} \mathrm{O}_{3}$ multilayer films are equivalent to their bulk oxide counterparts, whereas the $\mathrm{FeO}(111)$ monolayer film has different properties, when compared to bulk FeO. 


\subsection{Growth and structure of ultrathin FeO(111) films}

\subsubsection{FeO(111) layer-by-layer growth}

Figure 5.10 shows large area STM images of initial Fe-oxide growth stages on Pt(111). Submonolayer amounts of Fe were repeatedly deposited and subsequently oxidized for 2 min at $\mathrm{T}=870 \mathrm{~K}$. The clean $\mathrm{Pt}(111)$ substrate surface with terraces seperated by monoatomic steps is shown in (a). In (b) to (f) the oxide overlayer coverage increases from 0.7 to 2.2 ML. For all coverages, characteristic $\mathrm{FeO}(111) / \mathrm{Pt}(111)$ LEED patterns with satellites similar to the one displayed in Fig. 5.3b are observed. However, a closer inspection of the LEED patterns reveals small coverage dependent differences. They are due to small structural changes that lead to four slightly different $\mathrm{FeO}(111) / \mathrm{Pt}(111)$ coincidence structures, which all form unreconstructed surfaces terminated by a close-packed $\mathrm{O}$ layer. These structural changes also are reflected by different Moiré patterns visible in high-resolution STM images. They allow the identification of coverage dependent coincidence structures formed by the $\mathrm{FeO}(111)$ films and are discussed in Section 5.3.3.

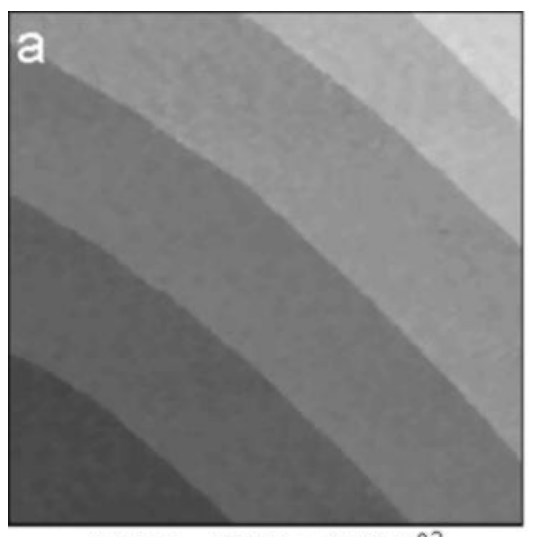

$0.0 \mathrm{ML}, 6000 \times 6000 \AA^{2}$

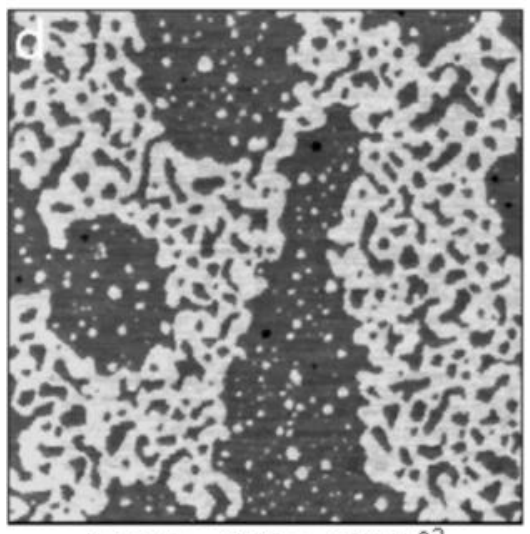

$1.5 \mathrm{ML}, 4000 \times 4000 \AA^{2}$

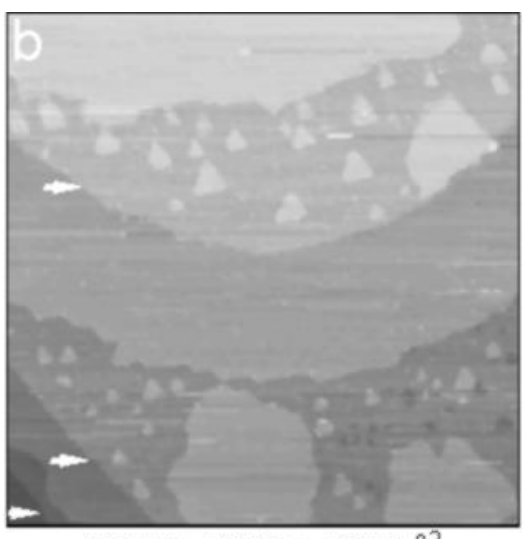

$0.7 \mathrm{ML}, 6000 \times 6000 \AA^{2}$

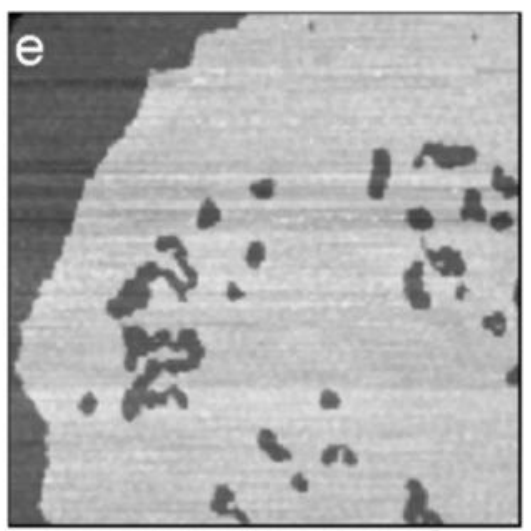

$1.9 \mathrm{ML}, 6000 \times 6000 \AA^{2}$

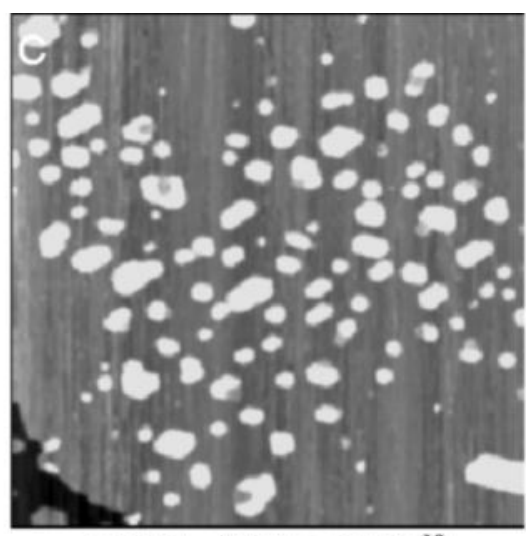

$1.2 \mathrm{ML}, 4000 \times 4000 \AA^{2}$

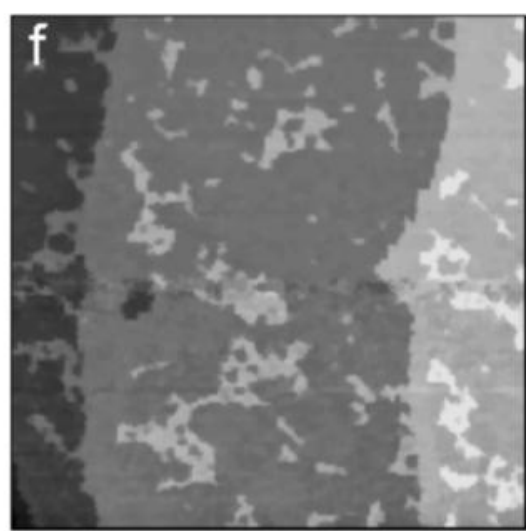

$2.2 \mathrm{ML}, 6000 \times 6000 \AA^{2}$

Fig. 5.10. Large scale STM images of initial Fe-oxide growth on $\mathrm{Pt}(111)$ for oxidation temperature of $870 \mathrm{~K}$. FeO(111) grows in layer-by-layer mode up to $2.5 \mathrm{ML}$ thickness. From [234].

In Fig. 5.10b about $70 \%$ of the Pt surface is covered by the first $\mathrm{FeO}$ layer. The terraces of the $\mathrm{Pt}$ substrate separated by monoatomic steps are marked by arrows. Starting at these steps, a closed overlayer always forms on the lower terrace, whereas overlayer islands and bare substrate regions coexist on the upper terrace near the step edges. Narrower Pt terraces, such as those in the lower left corner in Fig. 5.10b, are partly covered by a closed overlayer that started to form at the upward step edge. No overlayer islands form on the remaining bare parts. This indicates that a mobile Fe-oxide 
precursor is formed during the oxidation cycle, which prefers a downward step diffusion on the $\operatorname{Pt}(111)$ surface. No second layer islands have formed on top of the first layer at this growth stage. The Pt substrate steps get slightly rougher during $\mathrm{FeO}$ formation with more kink sites present. This step edge roughening can also be seen in Fig. 5.10e and $\mathrm{f}$.

Upon completion of the first $\mathrm{FeO}$ layer the first change in the atomic $\mathrm{FeO}(111)$ coincidence structure occurs (see Section 5.3.3.). The second $\mathrm{FeO}$ layer islands start to grow and have reached diameters from 50 to $400 \AA$ in Fig. 5.10c. Their edges run along the the [ $\overline{1} 10]$ and [01 $\overline{1}]$ directions of the $\mathrm{FeO}(111)$ overlayer. As the growth proceeds, the morphology of the second layer changes in a characteristic manner, which can be seen on the 1.5 ML thick film shown in Fig. 5.10d. In those regions where the coverage is sufficient for the coalescence of second layer islands, the surface morphology is characterized by the existence of vacancy islands that expose the first $\mathrm{FeO}$ layer underneath. The remaining surface parts are characterized by isolated small second layer islands. The step edges of both islands and vacancy islands run along the [ $\overline{1} 10]$ and [01 $\overline{1}]$ directions of $\mathrm{FeO}(111)$. At about 1.7 ML coverage, all second layer islands have coalesced and the whole surface is characterized by vacancy islands. Together with the formation of these vacancy islands in the second $\mathrm{FeO}$ layer the second change in the atomic $\mathrm{FeO}(111)$ coincidence structure takes place (see Section 5.3.3). In Fig. 5.10e, the second layer is almost completed. Third layer island growth does not start before completion of the second $\mathrm{FeO}$ layer. At this point, the third change in the $\mathrm{FeO}(111)$ coincidence structure takes place. In Fig. 5.10f, the coverage is $2.2 \mathrm{ML}$ and third FeO layer islands up to $500 \AA$ in diameter have formed. Note the difference in shape between the second and third layer islands at their initial growth stages, as visible by comparing Fig. 5.10c and $\mathrm{f}$. The largest obtainable FeO coverage was $2.5 \mathrm{ML}$. Around $2 \mathrm{ML}$ coverage $\mathrm{Fe}_{3} \mathrm{O}_{4}(111)$ islands start to grow. Their growth is discussed in Section 5.4 .

At $870 \mathrm{~K}$ oxidation temperature, Fe-oxide, thus, grows layer-by-layer onto $\mathrm{Pt}(111)$ up to $2.5 \mathrm{ML}$ thickness. Each layer consists of a FeO(111) bilayer. In the next Section, it will be shown that they are laterally expanded compared to bulk FeO. The Pt substrate steps underneath the oxide overlayer become slightly rougher. At oxidation temperatures around $1000 \mathrm{~K}$, only one $\mathrm{FeO}(111)$ bilayer can be grown on $\mathrm{Pt}(111)$. The high temperature growth is accompanied by strong changes of the Pt substrate morhoplogy, as discussed in [234].

\subsubsection{Atomic resolution STM and SPA-LEED measurements}

\section{(i) STM images}

The $\mathrm{FeO}(111)$ coincidence structures formed on $\mathrm{Pt}(111)$ were studied by atomic resolution STM and high-resolution SPA-LEED (Spot-Profile-Analysis LEED) measurements. The latter technique allows us to determine average surface lattice constants with high precision. By combining both techniques detailed models for the atomic arrangements in the $\mathrm{FeO}(111)$ films could be derived.

Fig. 5.11a displays a large scale $1000 \times 1000 \AA^{2}$ and a $55 \times 55 \AA^{2}$ atomic resolution STM image of the first coincidence structure formed at submonolayer coverages (structure 1). It exhibits a hexagonal surface lattice with a short-range periodicity of $3.11 \AA$ as determined precisely from the highresolution LEED intensity scan shown in Fig. 5.12 for $\Theta_{\mathrm{FeO}}=0.4$ ML. This short-range periodicity is modulated by a long-range periodicity, which creates a Moiré superstructure in the STM image. The large hexagonal unit cell of this Moiré superstructure can be defined by the brightest atomic features in the STM image marked in Fig. 5.11a, which do not line up with the atom rows on the $\mathrm{FeO}(111)$ surface. It has a lattice constant of $25 \AA$ and is rotated by $11^{\circ}$ against the small $(1 \times 1)$ surface unit cell on the oxide film. 

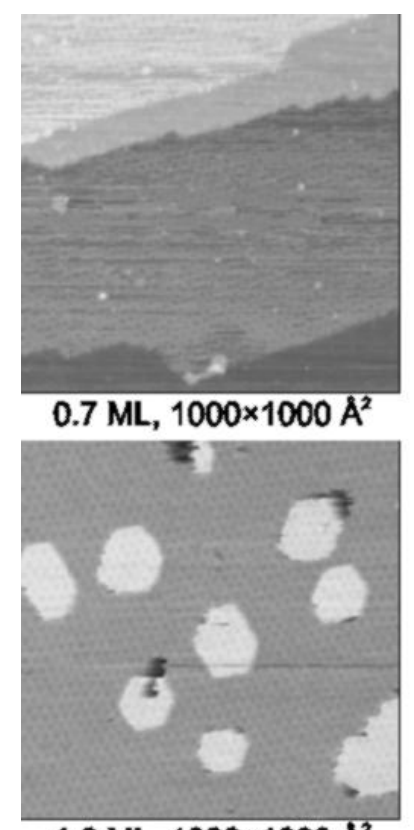

$1.2 \mathrm{ML}, 1000 \times 1000 \AA^{2}$
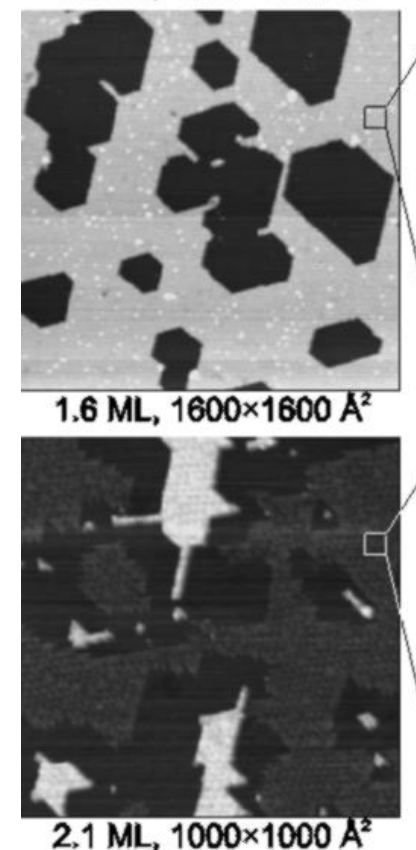
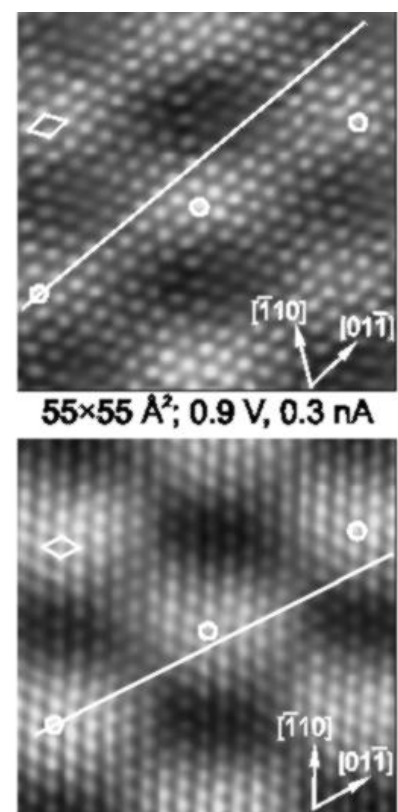

$55 \times 55 \AA^{2} ; 0.2 \mathrm{~V}, 0.5 \mathrm{nA}$

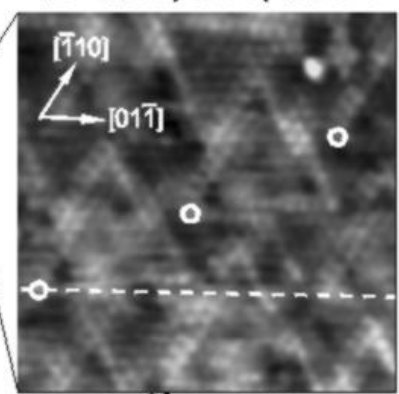

$85 \times 85 \AA^{2} ; 0.1 V, 0.7 n A$

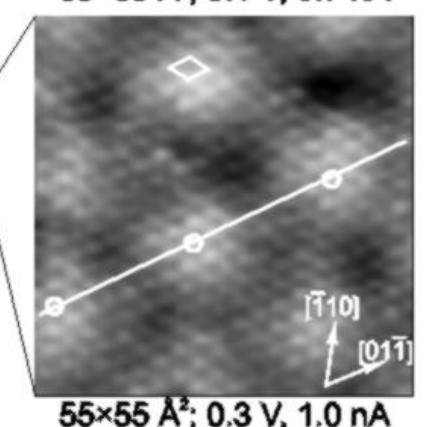

a)

\section{Structure 1}

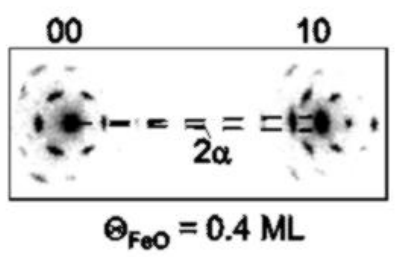

b)

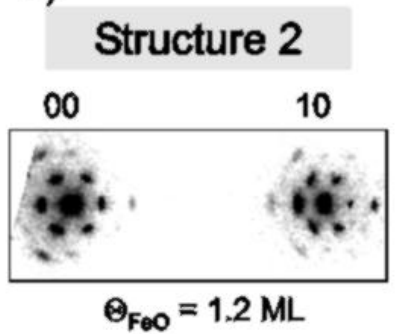

c)

\section{Structure 3(+2)}

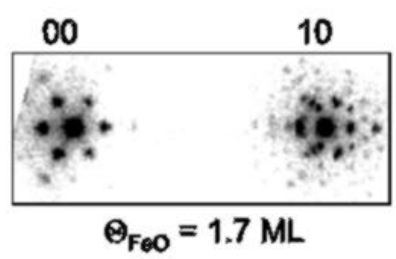

d)

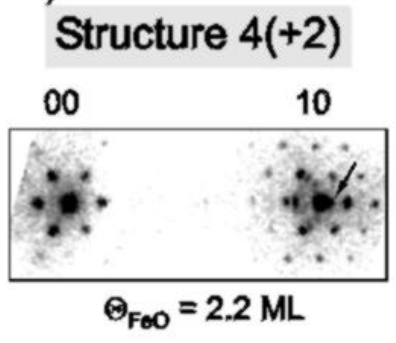

Fig. 5.11

Fig. 5.11. Four coincidence structures formed by $\mathrm{FeO}(111)$ on $\mathrm{Pt}(111)$ at coverages $\Theta_{\mathrm{FeO}}$ indicated. Large scale images on left show film morphologies, in middle corresponding atomic resolution STM images are presented, and on right grey scale intensity plots around the (00) and (10) beams of corresponding LEED patterns at $E=90 \mathrm{eV}$. Marked atoms and white lines indicate different Moiré angles of four structures. After [259, 261, 269] 


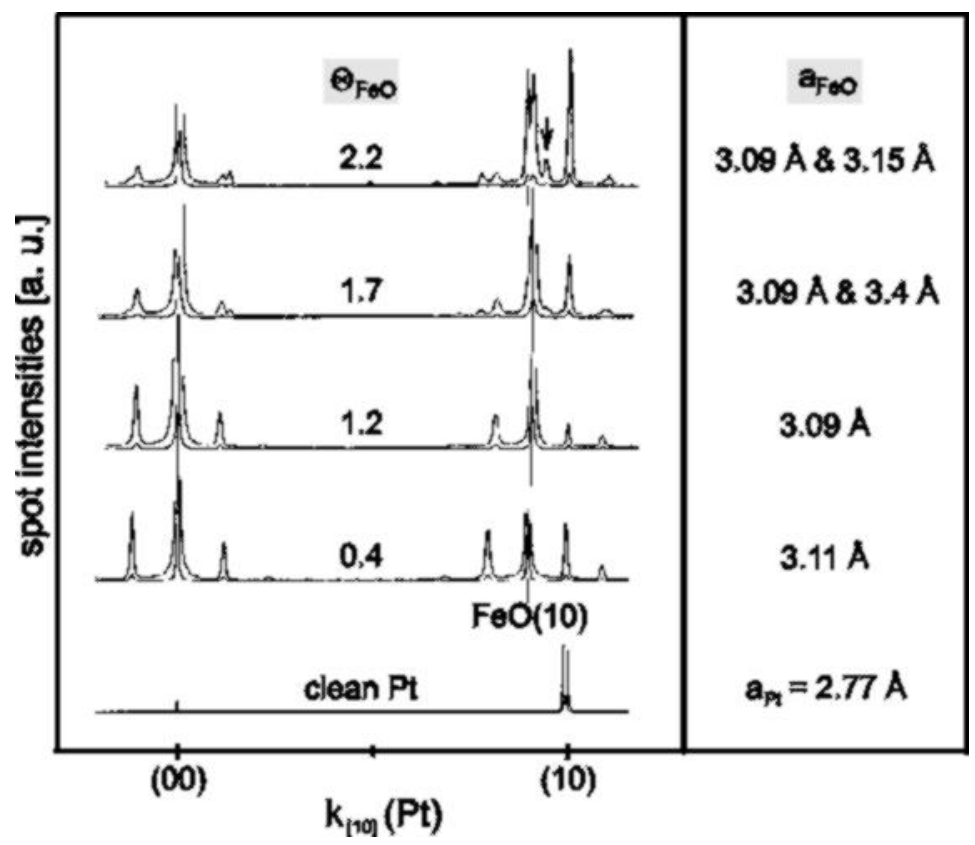

Fig. 5.12. SPA-LEED intensity line scans between (00) and (10) beams of clean $\mathrm{Pt}(111)$ surface and of $\mathrm{FeO}(111) / \mathrm{Pt}(111)$ coincidence structures displayed in Fig. 5.11. All curves are scaled in same way, line scans expanded by a factor of 10 are superimposed. Lattice constants $a_{\mathrm{FeO}}$ indicated on right were determined by comparison with (10) beam position of clean Pt(111). After [259].

Figure 5.13 shows a rigid model for this submonolayer structure 1. It consists of a laterally expanded $\mathrm{O}$ terminated $\mathrm{FeO}(111)$ bilayer on top of the $\mathrm{Pt}(111)$ surface. The Fe atoms are separated by $3.10 \AA$ and form rows that are rotated by $1.3^{\circ}$ against the underlying Pt atom rows along the [1 10$]$ and [01 $\overline{1}]$ directions. This corresponds to a lattice expansion of $2 \%$ compared to the cubic sodium chloride $\mathrm{FeO}$ bulk structure, which has an interatomic distance of $3.04 \AA$. The large superstructure cell indicated has a lattice constant of $25.4 \AA$. When starting from the left corner of this cell, the rotational mismatch of $\alpha=1.3^{\circ}$ between the $\mathrm{FeO}$ bilayer and the Pt surface lattice as well as the lateral $\mathrm{FeO}$ lattice constant of $\mathrm{a}_{\mathrm{FeO}}=3.10 \AA$ lead to an Fe coincidence site on the Pt surface after going eight Pt lattice spacings along the [01 $\overline{1}$ ] direction and two Pt lattice spacings along the [ $\overline{1} 10]$ direction. This site was chosen arbitrarily on top of a Pt surface atom in Fig. 5.13. In this way, the commensurate coincidence superstructure cell with a lattice constant of $\mathrm{a}_{\mathrm{coi}}=25.4 \AA$ is formed, which is rotated by $\beta=10.9^{\circ}$ against the $(1 \times 1)$ unit cell of the $\operatorname{Pt}(111)$ surface and by the Moiré angle $\alpha+\beta=12.2^{\circ}$ against the $\mathrm{FeO}(111)$ $(1 \times 1)$ unit cell. It also can be described by $(\sqrt{ } 84 \times \sqrt{ } 84) \mathrm{R} 10.9^{\circ}$ or by $\left(\begin{array}{cc}8 & 2 \\ -1 & 10\end{array}\right)$ superstructure cells using the Wood or matrix notation, respectively. The angle $\alpha+\beta=12.2^{\circ}$ and the lattice constant $\mathrm{a}_{\mathrm{FeO}}=3.10 \AA$ obtained from this model agree well with the Moiré angle of $11^{\circ}$ observed by STM and the $3.11 \AA$ lattice constant observed by LEED.

A similar model for an $\mathrm{FeO}(111)$ bilayer on $\mathrm{Pt}(111)$ was proposed by Galloway et al. for the first time [161], who observed the STM image, which we observe for coverages above 1 ML (structure 2 in Fig. 5.11). Electron-scattering quantum-chemistry theory calculations were performed to simulate this STM image. It turned out that the image contrast is not directly related to the surface topography, but is mainly due to electronic effects [163]. The bright regions in the Moire superstructure correspond to topographically low lying regions, where the first layer Fe atoms are located on Pt hollow sites. The dark regions in the STM Moiré pattern correspond to topographically high lying regions, where the Fe atoms are located on top of Pt atoms. For platinum STM tips, the atomic corrugation maxima occur over $\mathrm{O}$ atom positions. Based on these findings, we interpret the corrugation maxima in all atomic resolution STM images of Fig. 5.13 as the $\mathrm{O}$ atoms, which form a close-packed hexagonal surface layer. 


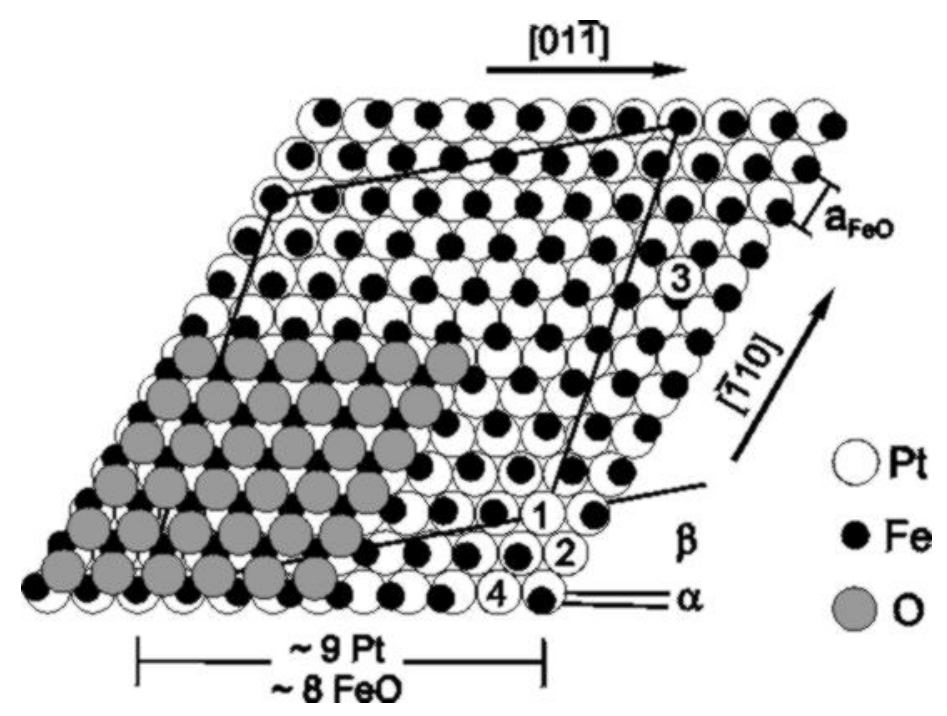

Fig. 5.13. Rigid model for four

$\mathrm{FeO}(111) / \mathrm{Pt}(111)$ coincidence structures.

$\mathrm{FeO}(111)$ bilayers have different lattice constants $a_{\mathrm{FeO}}$, rotation misfit angles against $\mathrm{Pt}$ surface lattice $\alpha$, and moiré angles $\alpha+\beta$. This leads to coincidence sites $1-4$, where Fe atoms are assumed to be located on top of Pt atoms.

Here coincidence structure 1 with its superstructure cell is shown. From [261].

\section{(ii) LEED patterns}

Figure 5.14 shows the LEED pattern of the low coverage $\mathrm{FeO}(111)$ film (structure 1), a schematic representation of it and a SPA-LEED grey scale intensity plot of the regions around the (00) and (10) beams. The first-order Pt substrate spots are still visible and occur at the same positions as on the clean surface (crosses in Fig. 5.14b). They correspond to the $\mathrm{Pt}(111)-(1 \times 1)$ surface unit cell with a lattice constant of $2.77 \AA$. The oxide film also forms a hexagonal LEED pattern, which is superimposed on the hexagonal $\mathrm{Pt}(111)$ LEED pattern. The first-order $\mathrm{FeO}(111)$ spots are located closer to the specular beam than the first-order Pt spots, because of its lager surface unit cellwith a lattice constant of about $3.1 \AA$ (large dots). The formation of the satellite diffraction spots around the (00) and $\mathrm{FeO}(10)$ beams (small dots) can be equally explained by multiple diffraction processes between the Pt substrate and the oxide overlayer as by diffraction at the large Moire superstructure unit cell of the buckled overlayer. Both descriptions lead to the same satellite spot positions that are given by linear combinations of $\mathrm{Pt}$ substrate and oxide overlayer surface reciprocal lattice vectors $\mathrm{g}(\mathrm{hk})_{\mathrm{Pt}}+\mathrm{g}(\mathrm{hk})_{\mathrm{FeO}}$, where $\mathrm{h}$ and $\mathrm{k}$ denote the indices of the integer diffraction spots [257]. From the weak satellite spot intensities, when compared to the substrate- and overlayer integer spot intensities, it can be concluded that multiple diffraction is the dominating mechanism. This indicates a small buckling in the oxide overlayer, which is in line with STM image simulations that revealed the atomic corrugations in the STM images to be mainly due to the local electronic surface structure and not to the surface topography [163].

In the multiple scattering picture, satellite spot 1 near the (00) beam in Fig. 5.14b, c, for example, is created by double diffraction described by the scattering vector sum $\mathrm{g}(10)_{\mathrm{Pt}}+\mathrm{g}(-10)_{\mathrm{FeO}}$. Spot 2 near the (10) beams is created by double diffraction described by $\mathrm{g}(01)_{\mathrm{Pt}}+\mathrm{g}(1-1)_{\mathrm{FeO}}$ and spot 3 by $\mathrm{g}(-11)_{\mathrm{Pt}}+\mathrm{g}(01)_{\mathrm{FeO}}$. Spot 4 is created by double diffraction $\mathrm{g}(0-1)_{\mathrm{Pt}}+\mathrm{g}(11)_{\mathrm{FeO}}$, spot 5 by $\mathrm{g}(-10)_{\mathrm{Pt}}+\mathrm{g}(20)_{\mathrm{FeO}}$ and spot 6 by $\mathrm{g}(-11)_{\mathrm{Pt}}+\mathrm{g}(2-1)_{\mathrm{FeO}}$. We also observe very weak spots, due to triple diffraction events, which are not visible in the LEED patterns as presented here.

For a non-rotated $\mathrm{FeO}(111)$ bilayer perfectly aligned to the Pt substrate, we expect all diffraction beams to be sharp. If domains with FeO bilayers rotated by different angles $\alpha$ coexist on the Pt surface, a characteristic broadening or splitting of some LEED spots is expected. The grey scale plot in Fig. $5.14 \mathrm{c}$ displays the (00) and (10) beams with their surrounding double diffraction satellite spots. Only 

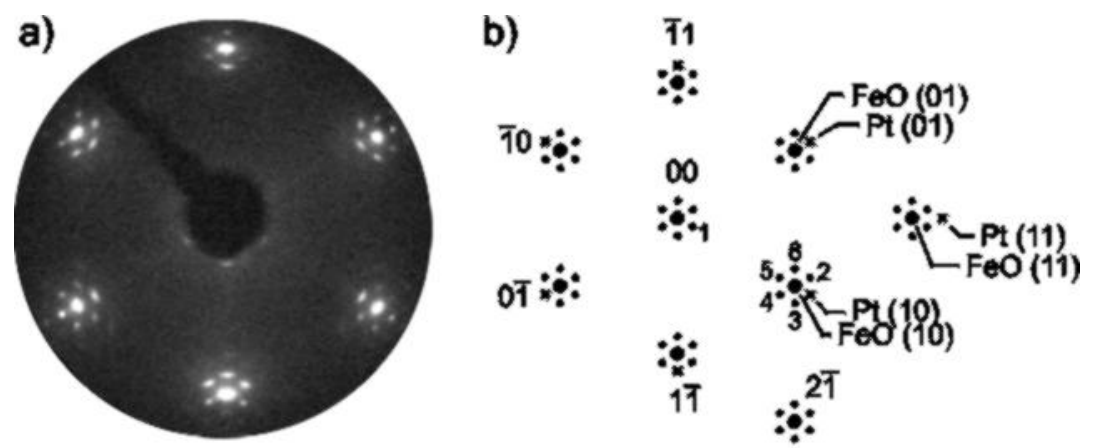

Fig. 5.14. (a) LEED pattern with satellite structure of $0.9 \mathrm{ML}$ thick FeO film on $\mathrm{Pt}(111)$ at $E=60 \mathrm{eV}$. (b) Schematic representation of this satellite LEED pattern. Crosses indicate Pt substrate spot positions, large dots first-order $\mathrm{FeO}$ integer spots, and small dots double diffraction satellite spots. (c) SPALEED gray-scale intensity plot of c)

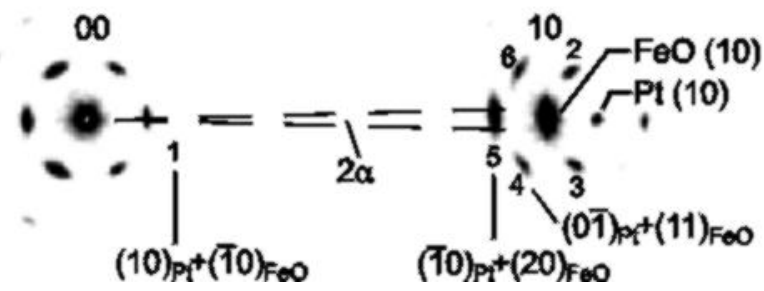

$\mathrm{FeO}(111) / \mathrm{Pt}(111)$ LEED pattern for $E=90 \mathrm{eV}$, displaying regions around (00) and (10) beams. Reciprocal surface lattice vectors involved in formation of some satellite spots are indicated. After [259, 269].

the $(00)$ and the $\mathrm{Pt}(10)$ beams are round, the $\mathrm{FeO}(10)$ beam is elongated perpendicular to the direction connecting the (00) and (10) beams. This is due to the coexistence of domains with different rotational mismatches between the $\mathrm{FeO}$ bilayer and the $\mathrm{Pt}$ substrate as discussed above. The rotation angle $\pm \alpha$ can occur in both directions, and is of the order of $1^{\circ}$, leading to domains rotated by $\alpha \approx \pm 1^{\circ}$, which produces a splitting or elongation of the $\mathrm{FeO}$ beams, depending on the resolution of the LEED system. The spot splitting is determined by the angle $2 \alpha$, as indicated in the grey scale plot in Fig. 5.14c. If several rotation angles are present a spot elongation is expected, which corresponds to LEED beam broadening on mosaic crystal surfaces [258]. The satellite spots around the $\mathrm{FeO}(10)$ beam are elongated according to the length of the involved overlayer scattering vectors parallel to the surface. Spots 2 and 3 are least elongated, because they are created by double diffraction, involving the shortest overlayer scattering vector $\mathrm{g}(10)_{\mathrm{FeO}}$, whereas spots 4 and 6 are more elongated, as they are created by double diffraction with the longer overlayer scattering vector $\mathrm{g}(11)_{\mathrm{FeO}}$. Spot 5 is elongated most, because the longest overlayer scattering vector $\mathrm{g}(20)_{\mathrm{FeO}}$ is involved. All these spots around the $\mathrm{FeO}(10)$ beam are elongated perpendicular to the direction connecting them with the $\operatorname{Pt}(10)$ spot, a consequence of the involved overlayer scattering vector directions.

\subsubsection{Models of coincidence structures}

For an oxidation temperature of $870 \mathrm{~K}$, the $\mathrm{FeO}$ films grow layer by layer upto a $2.5 \mathrm{ML}$ thickness. Four slightly different $\mathrm{FeO}(111)$ coincidence structures evolve with increasing $\mathrm{FeO}$ coverage $\Theta_{\mathrm{FeO}}$, which all exhibit similar satellite LEED patterns, as discussed in Section 5.3.1. Large scale STM images of the film morphologies, atomic resolution STM images and grey scale LEED intensity plots around the (00) and (10) beams of these structures are shown in Fig. 5.11. They differ with respect to the lattice constants of their $\mathrm{FeO}(111)-(1 \times 1)$ unit cells $a_{\mathrm{FeO}}$ and their coincidence superstructure unit cells $a_{c o i}$, their rotation misfit angles against the substrate surface lattice $\alpha$, and their Moire angles $\alpha+\beta$. The latter are visualized in the atomic resolution STM images by the marked atoms and the lines. These different lattice parameters lead to four different coincidence sites on the $\operatorname{Pt}(111)$ surface, which are numbered 1-4 in the rigid model depicted in Fig. 5.13. Table 5.1 summarizes the experimentally observed values for $a_{\mathrm{FeO}}, a_{c o i}, \alpha$, and $\alpha+\beta$ and the theoretical values obtained from the corresponding rigid models. The lattice constants $a_{\mathrm{FeO}}$ were determined from the high-resolution SPA-LEED intensity profiles shown in Fig. 5.12, the Moiré angles $\alpha+\beta$ from the atomic resolution STM images are 
displayed in Fig. 5.11. The misfit angles $\alpha$ could be estimated from the elongation of the $\mathrm{FeO}(10)$ beams in the LEED patterns in Fig. 5.11, and the coincidence superstructure lattice constants $a_{c o i}$ were determined both by the atomic resolution STM images and the corresponding satellite LEED spot positions.

\section{(i) Structure 1}

Structure 1 is formed at submonolayer coverages and was described in the previous section. The rigid model for this structure consists of an $\mathrm{FeO}(111)$ bilayer on top of the $\mathrm{Pt}(111)$ surface, where the coincidence site labeled 1 in Fig. 5.13 is reached after going eight Pt lattice spacings along the [01 $\overline{1}$ ] direction and two Pt lattice spacings along the [110] direction. This results in theoretical lattice constants of $a_{\mathrm{FeO}}=3.10 \AA$ and $a_{c o i}=25 \AA$, an rotation misfit angle against the Pt substrate of $\alpha=1.3^{\circ}$ and a Moiré angle of $12.2^{\circ}$. These values agree well with the corresponding experimentally observed values listed in Table 5.1.

\section{(ii) Structure 2}

Upon completion of the first layer structure 1 transforms into structure 2, which was observed by Galloway et al. [161]. The high-resolution LEED scan in Fig. 5.12 reveals a small shift of the FeO(10) beam away from the specular beam, due to reduction of the lateral lattice constant from $a_{\mathrm{FeO}}=3.11 \AA$ to $3.09 \AA$, when going from $\Theta_{\mathrm{FeO}}=0.4$ to $1.2 \mathrm{ML}$. The $\mathrm{FeO}(10)$ beam in Fig. $5.11 \mathrm{~b}$ is less elongated, when compared to structure 1. The Moiré angle in the STM image has decreased to $\alpha+\beta=5^{\circ}$, and the Moiré superstructure unit cell has a lattice constant of $a_{c o i}=26 \AA$. These values agree well with those of the rigid model in Fig. 5.13, where coincidence site 2 is reached after going nine Pt lattice spacings along the $[01 \overline{1}]$ direction and one Pt lattice spacing along the $[\overline{1} 10]$ direction, resulting in theoretical lattice constants of $\mathrm{a}_{\mathrm{FeO}}=3.09 \AA$ and $\mathrm{a}_{\mathrm{coi}}=26.4 \AA$, a misfit angle of $\alpha= \pm 0.6^{\circ}$ and an Moiré angle of $5.8^{\circ}$. On the first layer surface, this structure is observed until the second layer is completed, on the second layer surface it is observed up to coverages of about 1.5 ML.

\section{(iii) Structure 3 and 4}

At coverages between 1.5 and 2.0 ML, a new structure 3 is observed on the surface of the second layer islands, while structure 2 remains in the one layer thick regions. Figure 5.15 shows the regions around the (00) and (10) LEED beams observed at $\Theta_{\mathrm{FeO}}=1.7 \mathrm{ML}$. The new structure 3 spots, indicated by the grids, correspond to a $(8 \sqrt{ } 3 \times 8 \sqrt{ } 3) \mathrm{R} 30^{\circ}$ superstructure cell with respect to $\mathrm{Pt}(111)-(1 \times 1)$, which has a lattice constant of $\mathrm{a}_{\mathrm{coi}}=38.4 \AA$. Structure 2 spots are also visible. The shoulder on the left side of the $\mathrm{FeO}(10)$ beam in the line scan in Fig. 5.12 for $\Theta_{\mathrm{FeO}}=1.7 \mathrm{ML}$ is due to a structure 3 spot. In the rigid model of Fig. 5.13, this superstructure corresponds to coincidence site 3, which is reached after going $8 \mathrm{Pt}$ spacings along the [01 $]$ and [ $\overline{1} 10]$ directions each.

The STM image of structure 3 is not characterized by a honeycomb Moiré pattern, but by triangular features as can be seen in Fig. 5.11c. The triangles have side lenghts of about $35 \AA$ and occur with a periodicity of $38 \AA$ along directions rotated by $\beta= \pm 30^{\circ}$ to the [ $\left.01 \overline{1}\right]$ and [ $\left.\overline{1} 10\right]$ directions of the atom rows on $\mathrm{Pt}(111)$, which corresponds to the $(8 \sqrt{3} \times 8 \sqrt{ } 3) \mathrm{R} 30^{\circ}$ superstructure observed by LEED. The "triangle structure" in the second layer, thus, represents a coincidence structure with respect to the $\mathrm{Pt}(111)$ surface. A closer inspection reveals considerable short-range disorder with interatomic distances varying between 3.1 and $3.8 \AA$. Therefore, a rigid model with equally spaced atoms as shown in Fig. 5.13 is not a good description for structure 3. Nevertheless, the atomic resolution STM image 
indicates that coincidence site 3 is reached after going seven $\mathrm{FeO}$ lattice spacings along the [01 $\overline{1}$ ] direction and six $\mathrm{FeO}$ lattice spacings along the [110] direction. Together with the superlattice periodicity determined by LEED $\left(a_{c o i}=38.4 \AA\right)$ this results in an average lattice constant of $a_{\mathrm{FeO}}=3.40 \AA$ and a rotation angle between the overlayer atom rows and the Pt atom rows of $\alpha=-2.5^{\circ}$. The positions of the LEED spots marked by the arrows in Fig. 5.15 exactly correspond to this lattice constant and rotation misfit angle, which therefore can be considered as first-order spots of an average $(1 \times 1)$ unit cell of structure 3 . The Moiré angle between the superstructure unit cell and the $(1 \times 1)$ unit cell of structure 3 is $\alpha+\beta=27.5^{\circ}$, as indicated by the dotted line and the marked atoms in Fig. 5.11c.

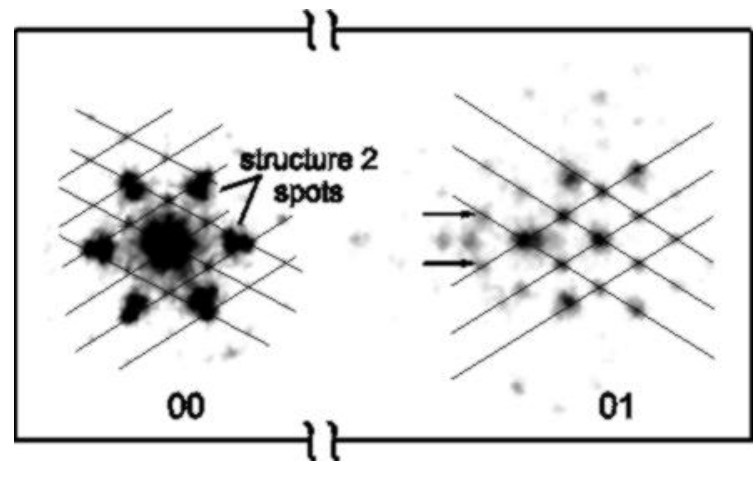

Fig. 5.15. SPA-LEED grey-scale intensity plots around (00) and (01) LEED beams for $\Theta_{\mathrm{FeO}}=1.7 \mathrm{ML}$. Fractional order spots of $(8 \sqrt{ } 3 \times 8 \sqrt{ } 3) \mathrm{R} 30^{\circ}$ coincidence structure 3 are indicated by line grids, double diffraction satellite spots of coexisting structure 2 are are also visible. Arrows mark first-order diffraction beams of a hexagonal lattice with lattice constant of $3.40 \AA$ and rotated by $\alpha= \pm 2.5^{\circ}$ against Pt substrate.

After $[259,269]$.

After completion of the second layer, structure 3 disappears completely and transforms into structure 4. At coverages between 2.0 and $2.5 \mathrm{ML}$, only structure 4 exists in the three layer thick regions, whereas structure 4 coexists with structure 2 in the in the two layer thick regions. The coexistence of structure 2 and 4 is evident from the two $\mathrm{FeO}(10)$ beams in the high-resolution LEED scan in Fig. 5.12 for $\Theta_{\mathrm{FeO}}=2.2 \mathrm{ML}$, which correspond to $\mathrm{FeO}$ lattice constants of $a_{\mathrm{FeO}}=3.09 \AA$ (structure 2) and $a_{\mathrm{FeO}}=3.15 \AA$ (structure 4). The spot marked by the arrow is due to $\mathrm{Fe}_{3} \mathrm{O}_{4}(111)$ islands that begin to form at this stage. The $\mathrm{FeO}(10)$ beam and the surrounding satellite spots of the LEED pattern of structure 4 in Fig. $5.11 \mathrm{~d}$ are not elongated, indicating no rotational misfit against the substrate lattice to exist anymore. The atomic resolution STM image of structure 4 reveals a Moiré superstructure with a lattice constant of $a_{c o i}=22 \AA$ that is not rotated against the FeO $(111)-(1 \times 1)$ unit cell. Seven $\mathrm{FeO}(111)$ lattice spacings can be seen within one superstructure period, which corresponds to a non-rotated $(8 \times 8)$ coincidence structure with respect to $\mathrm{Pt}(111)$, where $7 \mathrm{FeO}(111)$ lattice spacings fit onto $8 \mathrm{Pt}(111)$ lattice spacings as indicated by coincidence site 4 in the rigid model of Fig. 5.13. This model reveals $a_{\mathrm{FeO}}=3.16 \AA$ and $a_{c o i}=22.2 \AA$, in good agreement with the corresponding experimental values.

Since structures 3 and 4 have closely related superstructure unit cells and transform into each other with increasing coverage, a model for structure 3 in the second $\mathrm{FeO}$ layer with structure 4 in the first $\mathrm{FeO}$ layer underneath is proposed. An inspection of five different triangles in the atomic resolution STM image of structure 3 in Fig. 5.16a reveals that many $\mathrm{O}$ atom positions coincide within the error limits, but some definitely do not. The positions where at least three of the five atom positions coincided are mostly aligned along the rows marked by the lines in Fig. 5.16a, which form the triangles. These average $\mathrm{O}$ atom positions are shown as large black and gray circles in the model for structure 3 depicted in Fig. 5.16b. The open grey circles represent the first layer $\mathrm{O}$ atoms underneath. The large black atoms occupy hollow site positions with respect to the first $\mathrm{FeO}(111)$ bilayer, which correctly continue the $\mathrm{NaCl}$ type lattice, the grey atoms are on "wrong", mostly bridge positions. This leads to a more open packing with O-O distances between 3.1 and $3.8 \AA$ and an average value of 3.4 $\AA$. Pt substrate atoms are depicted as thin open circles in the lower half of the image. They are located 
below the triplets of small black circles, which mark the positions of the first layer Fe atoms located in threefold hollow sites of the Pt substrate (other Fe atoms omitted for clarity) and creating the $(8 \times 8)$ unit cell of structure 4 . These are the topographically lowest positions, but according to STM calculations [163], they represent the bright regions in the honeycomb STM patterns, due to electronic effects. The average positions of the triangles were arranged, so that their corners all have equivalent positions with respect to the Fe atom triplets, which in this way become located approximately halfway along the triangle sides. In fact, the STM image in Fig. 5.16a reveals the brightest protrusions in these regions, which supports the assumption of structure 4 in the first layer. Structure 3 is laterally expanded quite strongly, when compared to bulk $\mathrm{FeO}$, the reason for that will be discussed below.
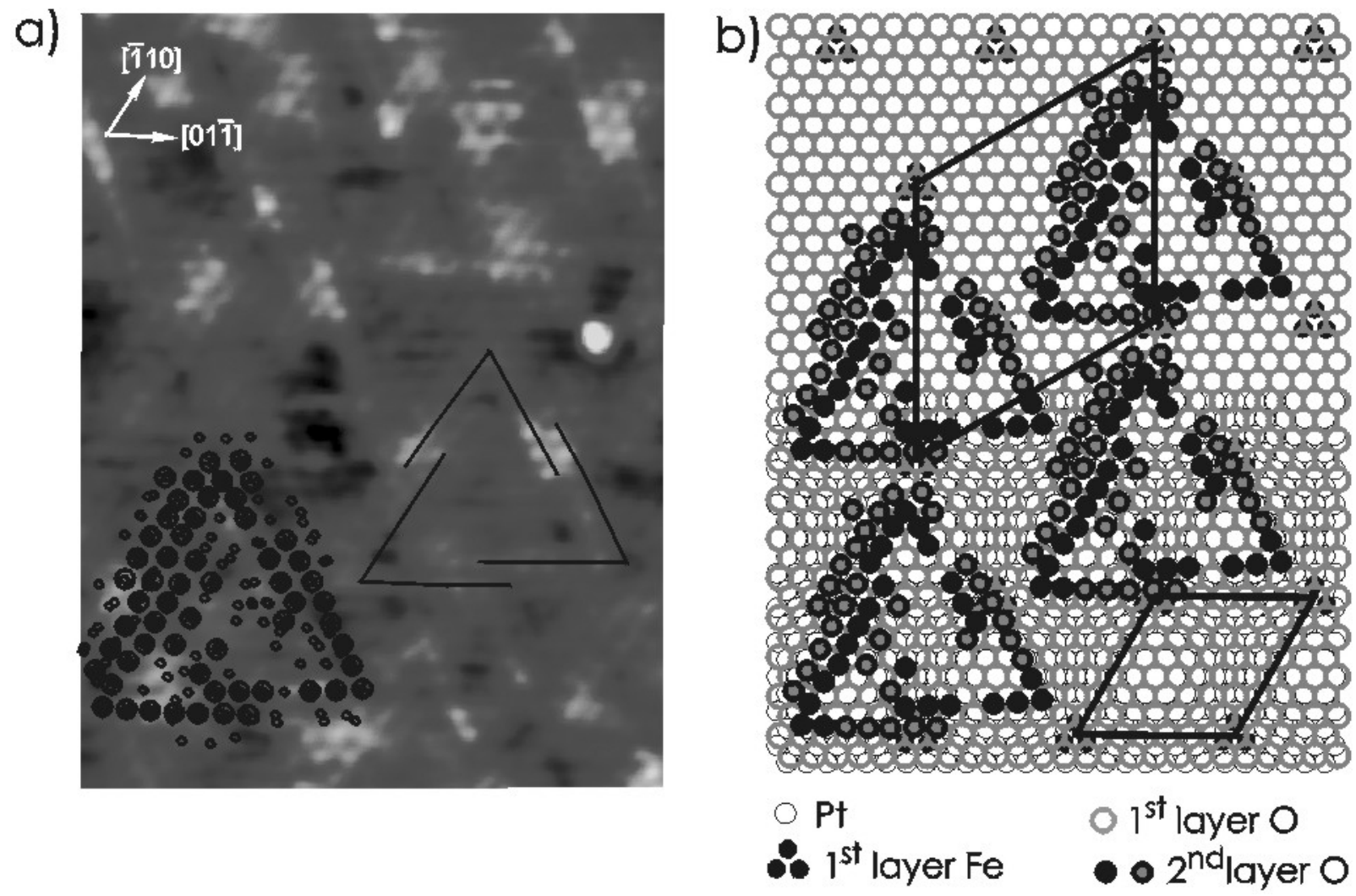

Fig. 5.16. (a) Atomic resolution $70 \times 90 \AA^{2}$ STM image of coincidence structure 3 . Triangles occur with a $(8 \sqrt{ } 3 \times 8 \sqrt{ } 3) \mathrm{R} 30^{\circ}$ periodicity relative to $\mathrm{Pt}(111)-(1 \times 1)$. Interatomic distances vary between 3.1 and 3.8 $\AA$ with average value of $3.4 \AA$. (b) Model for coincidence structure 3 formed in second FeO layer on top of first $\mathrm{FeO}$ layer with coincidence structure 4. Open black circles in lower half mark Pt atoms. Small black circle triplets denote first layer Fe atoms located on Pt hollow sites, which span $(8 \times 8)$ cell of structure 4 indicated by small hexagon. Large open grey circles mark $\mathrm{O}$ atoms in first layer. Large black and grey filled circles denote average $\mathrm{O}$ atom positions in second layer. Large hexagon indicates $(8 \sqrt{3} \times 8 \sqrt{ } 3) \mathrm{R} 30^{\circ}$ superstructure. After [261], supplemented by unpublished data. 
TABLE 5.1. Lattice parameters of rigid models for $\mathrm{FeO}(111) / \mathrm{Pt}(111)$ coincidence structures 1-4. Coverage ranges $\Theta_{\mathrm{FeO}}$ of their appearence are listed. Large coincidence superstructure unit cells are given in matrix and Wood notations, referred to $\mathrm{Pt}(111)-(1 \times 1) . a_{\mathrm{FeO}}$ is lattice constant of $\mathrm{FeO}(111)-$

$(1 \times 1)$ unit cell and $a_{\text {coi }}$ that of coincidence superstructure cell. $\alpha$ is rotation misfit angle between $\mathrm{FeO}(111)$ bilayer and $\mathrm{Pt}(111)$ surface lattice, $\alpha+\beta$ is Moiré angle observed in STM images. Underlined numbers denote experimentally observed values, other numbers theoretically expected ones. After [259].

\begin{tabular}{|c|c|c|c|c|c|c|c|}
\hline No. & $\Theta_{\mathrm{FeO}}[\mathrm{ML}]$ & \multicolumn{2}{|c|}{ coincidence structure } & $a_{\mathrm{FeO}}[\AA]$ & $a_{c o i}[\AA]$ & $\alpha$ & $\alpha+\beta$ \\
\hline 1 & $0-1 \mathrm{ML}$ & $\left(\begin{array}{cc}8 & 2 \\
-2 & 10\end{array}\right)$ & $(\sqrt{84} \times \sqrt{84}) \mathrm{R} 10.9^{\circ}$ & $\begin{array}{c}3.102 \\
\underline{3.11} \\
\end{array}$ & $\begin{array}{c}25.4 \\
\underline{25}\end{array}$ & $\begin{array}{l}1.3^{\circ} \\
1-1.5^{\circ} \\
\end{array}$ & $\begin{array}{c}12.2^{\circ} \\
\underline{11^{\circ}} \\
\end{array}$ \\
\hline 2 & $1-2 \mathrm{ML}$ & $\left(\begin{array}{cc}9 & 1 \\
-1 & 10\end{array}\right)$ & $(\sqrt{91} \times \sqrt{91}) \mathrm{R} 5.2^{\circ}$ & $\begin{array}{c}3.093 \\
\underline{3.09} \\
\end{array}$ & $\begin{array}{c}26.4 \\
\underline{26}\end{array}$ & $\begin{array}{c}0.6^{\circ} \\
\text { small }\end{array}$ & $\begin{array}{l}5.8 \\
5^{\circ} \\
\end{array}$ \\
\hline 3 & $1.5-2 \mathrm{ML}$ & $\left(\begin{array}{cc}8 & 8 \\
-8 & 16\end{array}\right)$ & $(8 \sqrt{3} \times 8 \sqrt{3}) \mathrm{R} 30^{\circ}$ & $\begin{array}{c}3.406 \\
\underline{3.4^{*}}\end{array}$ & $\begin{array}{c}38.4 \\
\underline{38}\end{array}$ & $\underline{-}-5^{\circ}$ & $\begin{array}{r}27.5^{\circ} \\
\underline{28^{\circ}}\end{array}$ \\
\hline 4 & $2-2.5 \mathrm{ML}$ & $\left(\begin{array}{ll}8 & 0 \\
0 & 8\end{array}\right)$ & $(8 \times 8)$ & $\begin{array}{c}3.166 \\
\underline{3.15}\end{array}$ & $\begin{array}{c}22.2 \\
22 \\
\end{array}$ & $\begin{array}{c}0^{\circ} \\
\text { small } \\
\end{array}$ & $\begin{array}{c}0^{\circ} \\
\text { small } \\
\end{array}$ \\
\hline
\end{tabular}

* average value

\section{(iv) Interlayer distances}

All $\mathrm{FeO}(111)$ coincidence structures formed on $\mathrm{Pt}(111)$ are laterally expanded, when compared to the $\mathrm{FeO}$ bulk structure. These lateral expansions are accompanied by reductions of the Fe-O interlayer spacings (Table 5.2). For structure 2 in the first layer, an interlayer distance of $0.68 \AA$ was deduced from photoelectron diffraction measurements [260] and from STM measurements in conjunction with theoretical image simulations [164], 45\% smaller than the FeO bulk value of $1.25 \AA$. In this work, a step height of $2.1 \AA$ was measured by STM for second layer islands with structure 2 located on first layer regions with structure 2, as well as for third layer islands with structure 4 located on second layer regions with structure 4 . This results in an Fe-O interlayer distance of $1.05 \AA, 16 \%$ smaller than the $\mathrm{FeO}$ bulk value. From the lateral lattice constants and the interlayer distances, the Fe-O bond lengths were calculated, as listed in Table 5.2. They all are reduced, when compared to the FeO bulk value (see Section 5.3.5).

\subsubsection{Defect structures}

At submonolayer coverages, coincidence structure 1 (Fig. 5.11a) is formed. In the rigid model, the $\mathrm{FeO}(111)$ bilayer is laterally expanded and rotated by $1.3^{\circ}$ against the Pt surface lattice, creating a Moiré superstructure with a defined lattice constant and Moiré angle. However, close inspection of the atomic resolution STM images shows that the superstructure period and the Moiré angles vary slightly. The rigid coincidence structure models are only an approximate description for the $\mathrm{FeO}(111)$ films. Furthermore, the overlayer atom rows do not form exactly straight lines, but are wiggled, which can be seen in the atomic resolution STM image of Fig. 5.11a. Slightly different rotational mismatches between the $\mathrm{FeO}(111)-(1 \times 1)$ unit cells and the Pt substrate are created in this way. 
TABLE 5.2. Experimentally measured lateral lattice constants $\mathrm{aFeO}$ and $\mathrm{Fe}-\mathrm{O}$ interlayer distances in the first three $\mathrm{FeO}$ layers. Fe-O interatomic distances were deduced from these values. From [261].

\begin{tabular}{|c|c|c|c|c|}
\hline Layer & Structure & $\mathrm{a}_{\mathrm{FeO}}[\AA]$ & $\begin{array}{c}\mathrm{Fe}-\mathrm{O} \\
\text { Interlayer distance }[\AA]\end{array}$ & $\begin{array}{c}\text { Fe-O } \\
\text { interatomic distance }[\AA]\end{array}$ \\
\hline $1^{\text {st }}$ & 1 & 3.11 & & 1.91 \\
\hline $1^{\text {st }}$ & 2 & 3.09 & 0.68 & 2.10 \\
\hline $2^{\text {nd }}$ & 2 & 3.09 & 1.05 & 2.10 \\
\hline $2^{\text {nd }}$ & 3 & $3.40^{*}$ & $<1.05$ & 2.16 \\
\hline $3^{\text {rd }}$ & 4 & 3.15 & 1.05 & 1.25 \\
\hline bulk & & 3.04 & \multicolumn{3}{c}{} \\
\hline
\end{tabular}

* average value

The existence of different rotation misfit angles is also evident from the $1000 \times 1000 \AA^{2}$ image of an 0.9 ML thick FeO film, shown in Fig. 5.17, which shows several defect structures. The honeycomb Moiré superstructure is clearly visible. The dark regions without honeycomb pattern are uncovered $\mathrm{Pt}$ areas. A monoatomic Pt step separating two FeO covered terraces is visible in the upper left corner. The Pt step height of $2.3 \AA$ is measured. Two domain boundaries meet at the upper left of the image. Lateral shifts between the honeycomb coincidence superstructure cells are found at such domain boundaries, as indicated by arrow 1. Formerly separated $\mathrm{FeO}$ island have grown together at such boundaries. The region above the domain boundary, marked by arrow 1, shows a well-ordered superstructure without visible defects. The superstructure cell direction changes by $3^{\circ}$ at the position marked by arrow 2 . Arrow 4 indicates a $7^{\circ}$ direction change of the rows formed by the superstructure cells, which can be explained by the coexistence of structures 1 and 2 , indicating that structure 2 already exists at this stage. The $3^{\circ}$ direction change indicates the existence of superstructures with different rotation misfit angles. In the lower part of the image, marked by number 3 , several honeycomb superstructure rows end in small areas of uncovered Pt. Two honeycomb rows formerly separated by a row in between them move together at these points. They are inclined by small angles with respect to each other. The honeycomb row end points are located along a tilt grain boundary, where slightly inclined superstructure rows meet. This tilt grain boundary in an epitaxial monolayer film is the two-dimensional analogy to the well-known tilt grain boundaries in three-dimensional crystals [262].

\subsubsection{Discussion}

If heteroepitaxial growth takes place close to equilibrium conditions, the growth mode depends on the surface free energies of the substrate $\gamma_{s}$ and the film $\gamma_{f}$ as well as on the interfacial energy $\gamma_{i, n}$ [263]. The interfacial energy $\gamma_{i, n}$ depends on the specific chemical interaction between film and substrate atoms at the interface. When a material grows pseudomorphic on a substrate with lattice mismatch, $\gamma_{i, n}$ also contains the elastic energy of the strained film, which increases with film thickness. Then $\gamma_{i, n}$ depends on the number of layers $n$. The surface energy of the strained film $\gamma_{f}$ might deviate from the corresponding surface energy of an unstrained semi-infinite crystal, but $\gamma_{f}$ does not depend on the film thickness for metal and semiconductor materials studied so far. However, the energy of polar oxide surfaces can depend on the film thickness, as discussed below. In this case, the energy of the system can be written as

$$
\Delta \gamma_{n}=\gamma_{f, n}+\gamma_{i, n}-\gamma_{s}
$$


with $\gamma_{f, n}$ denoting the film surface energy depending on the number of layers $n$. A layer-by-layer growth (Frank-van der Merve mode) is only possible, if $\Delta \gamma_{n}<0$, for all $n$. If $\Delta \gamma_{n}>0$ already for the first layer $n=1$, a three-dimensional film growth takes place from the beginning (Volmer-Weber mode). If $\Delta \gamma_{n}>0$, for $n>1$, the first $n$-1 layers grow layer-by-layer, before a three-dimensional growth starts (i.e. Stranski-Krastanov growth).

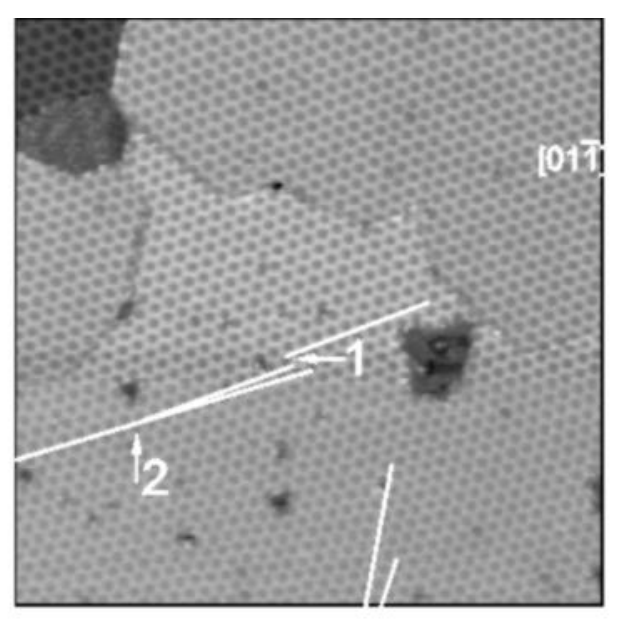

Fig. 5.17. $1000 \times 1000 \AA^{2} \mathrm{STM}$ image of $0.9 \mathrm{ML}$ $\mathrm{FeO}(111)$ film on $\mathrm{Pt}(111)$. Honeycomb Moiré superstructure is visible. Indicated defects are discussed in text. $\mathrm{U}_{\mathrm{t}}=0.5 \mathrm{~V}, \mathrm{I}_{\mathrm{t}}=0.2 \mathrm{nA}$. From

[259].

The first Fe-oxide layer completely wets the Pt surface. Obviously its surface free energy $\gamma_{f, 1}$ is lower than that of the $\operatorname{Pt}(111)$ substrate surface $\gamma_{s}$, and the interfacial energy $\gamma_{i, 1}$ is also small. This is an expected behavior, since many metal-oxides have lower surface energies than metals [129], which is the reason why most metals form islands or clusters on oxide substrates [264]. The first FeO layers are laterally expanded, when compared to bulk FeO. This increases the mismatch beyond that of bulk $\mathrm{FeO}(3.04 \AA)$, which is $10 \%$ compared to $\mathrm{Pt}(111)(2.77 \AA)$. Therefore, the initial Fe-oxide growth is not pseudomorphic. Three slightly different $\mathrm{FeO}(111)$ coincidence structures (1, 2, and 4) form stable interface configurations at the corresponding overlayer coverages. This indicates that the energetics of the chemical interaction at the $\operatorname{Pt}(111)-\mathrm{FeO}(111)$ interface does not change much, if the interface geometry varies slightly, which also is confirmed by the coexistence of slightly different rotation misfit angles against the Pt surface (Fig. 5.17). Although there exist preferred coincidence sites, creating the observed LEED patterns, other sites can also occur. The total energy of the system is, thus, dominated by the surface energies $\gamma_{f, n}$ and the elastic energies of the strained films, which are contained in $\gamma_{i, n}$.

For an oxidation temperature of $870 \mathrm{~K}$, the $\mathrm{FeO}(111)$ films grow layer-by-layer up to a maximum thickness of $2.5 \mathrm{ML}$, when a three-dimensional growth of $\mathrm{Fe}_{3} \mathrm{O}_{4}(111)$ islands starts as is discussed in the next section. This represents a Stranski-Krastanov growth mode for Fe-oxides on $\mathrm{Pt}(111)$, if we expand this growth mode terminus to the present case with two different oxide phases involved, $\mathrm{FeO}$ and $\mathrm{Fe}_{3} \mathrm{O}_{4}$, which have closely related crystal structures. The Stranski-Krastanov growth of metal and semiconductor systems, studied so far, begins with a pseudomorphic layer-by-layer growth that is limited by the elastic film energy. This elastic energy increases up to a critical film thickness, then a three-dimensional growth of unstrained islands with dislocations at the substrate-overlayer interface or of coherently strained islands with limited sizes becomes favorable [265]. The layer-by-layer growth of $\mathrm{FeO}(111)$ is limited by a different mechanism, when compared to metals and semiconductors. If we consider the interface energy to be constant, each of the four different $\mathrm{FeO}(111)$ coincidence structures 
must reflect a minimum total energy arrangement balancing the elastic energies in the strained films and the surface energies for each coverage. The lateral expansion increases in the second and third $\mathrm{FeO}$ layers, when compared to the first layer expansion (see Table 5.1), an unusual behaviour as one would expect the film to assume the $\mathrm{FeO}$ bulk structure with increasing thickness. The lateral expansions are accompanied by reduced interlayer spacings (Table 5.2). An inward relaxation of the outermost atomic planes is expected for polar metal-oxide surfaces [6], as discussed in Section 5.5 for the $\mathrm{Fe}_{3} \mathrm{O}_{4}$ surface, but not for the interlayer distance of the deeper lying first and second bilayers, as observed here. However, these relaxations reduce the electrostatic $\mathrm{FeO}(111)$ surface energy, which increases with the number of layers $n$. The layer-by-layer growth for $\mathrm{FeO}$ is limited to a maximum thickness of $2.5 \mathrm{ML}$, mainly because of the film surface energy $\gamma_{f, n}$. which increases with film thickness.

Figure 5.18 illustrates schematically one $(\mathrm{a}, \mathrm{c})$ and two $(\mathrm{d}, \mathrm{f})$ bilayer thick films of an ionic material on top of a metal substrate. Each bilayer consists of two oppositely charged atomic planes as for $\mathrm{FeO}(111)$ on $\mathrm{Pt}(111)$. The $\mathrm{FeO}(111)$ films form polar unreconstructed surfaces, terminated by negatively charged close-packed $O$ layers, which represent type 3 surfaces according to the classification of Tasker. As discussed in Section 2.8, each bilayer increases the surface potential $V(z)$, as indicated by the solid lines in Fig. $5.18 \mathrm{~b}$ and e for the case of unrelaxed films. For a bulk crystal, this leads to an infinite surface energy, for thin films the surface energy $\gamma_{f, n}$ increases with the number of layers $n$ to finite values. Since the potential step within each bilayer is proportional to the interlayer distance, the surface energy of the films can be decreased by relaxations that reduce the interlayer distances. This situation is depicted in Fig. 5.18c and $\mathrm{f}$ and by the broken lines in Fig. 5.18b and e. The electrostatic surface potential is, thus, the driving force for the reduction of the Fe-O interlayer distances in the. Since reduced interlayer spacings would reduce the Fe-O bond lengths, the films respond by increasing their lateral lattice constants. The relaxing $\mathrm{O}$ atoms squeeze the $\mathrm{Fe}$ atoms underneath apart, leading to lateral lattice constants of $a_{\mathrm{FeO}}=3.11 \AA$ and $3.09 \AA$ for structures 1 and 2 in the first bilayer, respectively. In the second layer, the lateral expansions become even larger, because the surface potential increases considerably as the second layer grows, leading to structure 3 $\left(a_{\mathrm{FeO}}=3.4 \AA\right)$ and structure $4\left(a_{\mathrm{FeO}}=3.15 \AA\right)$. The resulting Fe-O bond lenghts in the second and third $\mathrm{FeO}$ layers are $2.1 \AA$, slightly smaller than the $\mathrm{FeO}$ bulk value of $2.16 \AA$ (see Table 5.2). The interlayer distance for structure 2 in the first layer is $0.68 \AA$, resulting in an Fe-O bond length of only $1.91 \AA$. This is considerably smaller than the $\mathrm{FeO}$ bulk value and corresponds to a value expected for adsorbed $\mathrm{O}$, as observed for $\mathrm{O}$ on rhodium [266] and ruthenium [267]. This indicates a reduced ionic charge in the first bilayer, which must be induced by a charge redistribution within the overlayer, as indicated by the $\delta^{-}$in Fig. 5.18f. As discussed in Section 2.8, such a charge redistribution further decreases the surface energy according to the dotted potential line in Fig. 5.18e.

Since the second bilayer considerably increases the surface energy, the system tries to avoid its formation and forms a slightly compressed first bilayer with structure 2 before the first layer gets completed. The second layer then starts to grow with structure 2, and the surface energy of the film strongly increases with the second layer coverage. This is the driving force for the transformation into structure 3 in the second layer, which is located on top of structure 4 in the first layer. Structure 3 is extremely expanded with O-O distances up to $3.8 \AA$ and an average lattice constant of $3.4 \AA$. The top layer $\mathrm{O}$ atoms relax deeply into the $\mathrm{Fe}$ layer, leading to a strongly reduced $\mathrm{Fe}-\mathrm{O}$ interlayer distance and, thus, a much smaller surface energy. The $\mathrm{Fe}$ and $\mathrm{O}$ atoms of the second bilayer even leave their correctly coordinated sites with respect to the underlying structure 4 . Before the second layer is completed, structure 3 transforms into the more compressed structure 4, because the system again tries to avoid the formation of the next layer. Third layer islands with structure 4 then start to grow. It can be seen in the large scale STM image in Fig. 5.11d that the even more compressed structure 2 forms around these third layer islands. The system tries to incorporate as much material as possible into the first two bilayers, in order to avoid the third layer growth, because it would increases the surface 
energy of the film so strongly. More than a FeO coverage of $2.5 \mathrm{ML}$ is not possible, because growth of $\mathrm{Fe}_{3} \mathrm{O}_{4}$ islands then becomes favorable.

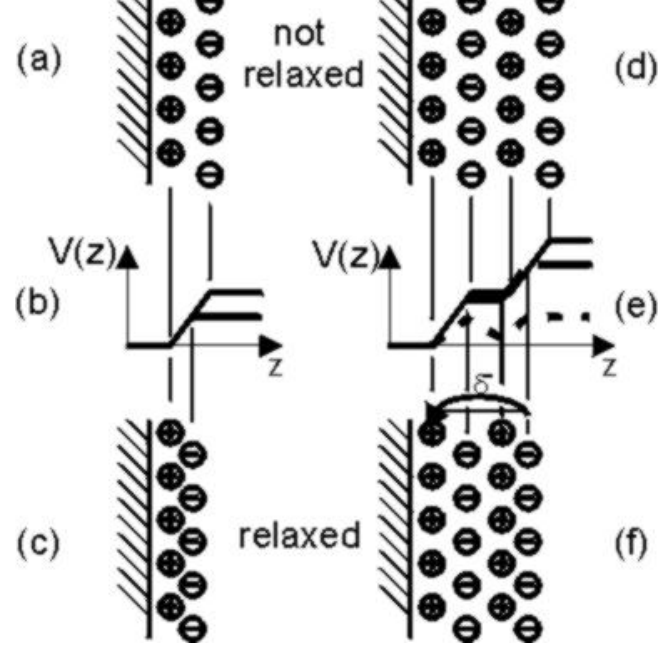

Fig. 5.18. Schematic model of one and two bilayer thick films of ionic material on top of metal substrate in side view representation. Each bilayer consists of two oppositely charged planes.

Electrostatic potential variations $V(r)$ for unrelaxed and relaxed overlayers are indicated by solid and broken lines, respectively. Dotted line indicates variation after charge redistribution of $\delta^{-}$ has occured within overlayer. From [261].

In summary, Fe-oxide grows in a Stranski-Krastanov mode onto $\mathrm{Pt}(111)$ involving two oxide phases with closely related crystal structures, $\mathrm{FeO}$ and $\mathrm{Fe}_{3} \mathrm{O}_{4}$. The initial $\mathrm{FeO}$ layer-by-layer growth is limited to a maximal thickness of $2.5 \mathrm{ML}$, mainly because of the $\mathrm{FeO}(111)$ surface energy, which increases with the number of layers. The reduction of the electrostatic energy of the polar $\mathrm{FeO}(111)$ surface is also the driving force for the observed strong relaxations. Four different coincidence structures, which all have laterally expanded lattice constants and reduced interlayer distances are formed up to $2.5 \mathrm{ML}$ coverage. The oxide then continues to grow by formation of three-dimensional $\mathrm{Fe}_{3} \mathrm{O}_{4}(111)$ islands. This growth mechanism, ruled by electrostatic surface energies, may well be involved in epitaxial growth of many ionic materials along polar crystal directions.

\subsection{Growth of $\mathrm{Fe}_{3} \mathrm{O}_{4}(111)$ and $\alpha-\mathrm{Fe}_{2} \mathrm{O}_{3}(0001)$ multilayer films}

\subsection{1. $\mathrm{Fe}_{3} \mathrm{O}_{4}$ growth at $\mathrm{T}=870 \mathrm{~K}$}

In order to study the further film growth, further Fe was deposited and oxidized. For an oxidation temperature of $870 \mathrm{~K}, \mathrm{Fe}$ quantities corresponding to $1 \mathrm{ML}$ were chosen. Consequently, it took a large number of growth cycles to form thick $\mathrm{Fe}_{3} \mathrm{O}_{4}$ films. Although this preparation method and the surface characterization cycles in between took upto several days, contamination by $\mathrm{C}$ or $\mathrm{OH}$ groups was never observed since these contaminations are readily removed during each oxidation cycle. Also, thick films can be cleaned simply by oxidation. For studies on the surface of thick films, a large amount of Fe was deposited in one cycle and oxidized for correspondingly longer periods. The same final film quality can be reached in this way.

The $1 \times 1 \mu \mathrm{m}^{2}$ images in Fig. 5.19 display the Fe-oxide growth at an oxidation temperature of $870 \mathrm{~K}$ after about $2 \mathrm{ML}$ of $\mathrm{FeO}$ have grown layer by layer. Figure 5.19a is similar to the film shown in Fig. 5.10f with the second $\mathrm{FeO}$ layer completed and the third $\mathrm{FeO}$ layer islands having formed. Additional deposition led to several islands with nearly triangular basal planes. They are 20-25 $\AA$ high and extend laterally over 2000 to $3000 \AA$. At this point, the LEED pattern Fig. $5.19 \mathrm{e}$ is observed. It is a superposition of the $\mathrm{FeO}(111) / \mathrm{Pt}(111)$ satellite LEED pattern and the $\mathrm{Fe}_{3} \mathrm{O}_{4}(111)$ LEED pattern shown in Fig. 5.3. The brightest spots are split, as a result of the different average interatomic distances within the $\mathrm{O}$ (111) planes of the two oxides, as discussed in Section 5.2. High-resolution LEED 
measurements reveal exactly the bulk lattice spacing of $\mathrm{Fe}_{3} \mathrm{O}_{4}$, which shows that (111) oriented islands with the $\mathrm{Fe}_{3} \mathrm{O}_{4}$ bulk structure have formed.
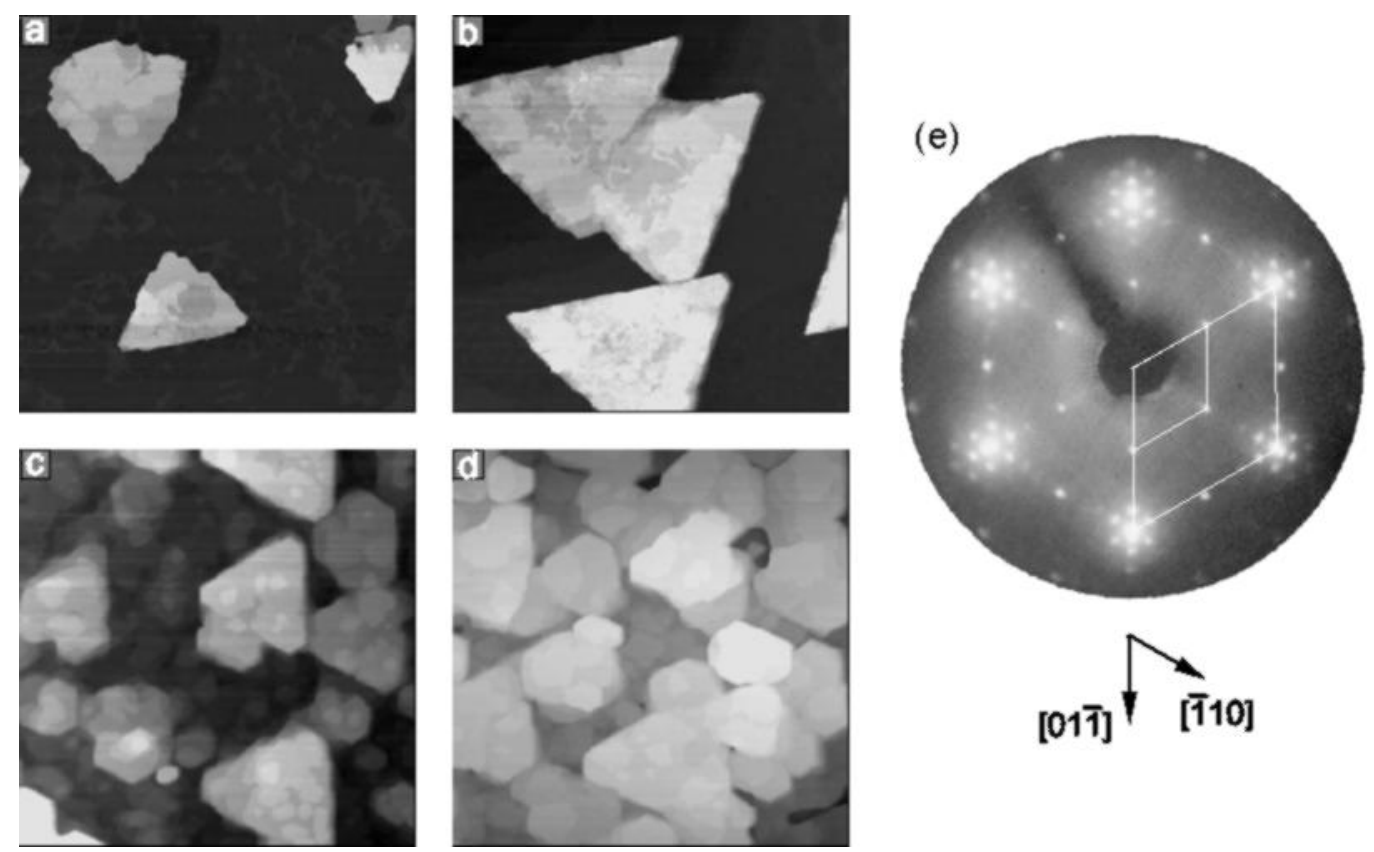

Fig. 5.19. (a)-(d): $1 \times 1 \mu \mathrm{m}^{2}$ STM images of consecutive $\mathrm{Fe}_{3} \mathrm{O}_{4}(111)$ island growth stages for oxidation temperature of $870 \mathrm{~K}$. Initially number of islands does not increase but island sizes do. Island heights are 20-25 $\AA$ in (a) and 40-50 $\AA$ in (b) and (c). In (c) new islands have nucleated between large ones, in

(d) all islands have coalesced forming $\mathrm{Fe}_{3} \mathrm{O}_{4}(111)$ multilayer film at least $100 \AA$ thick. (e): LEED pattern of island film (a) at $E=60 \mathrm{eV}$. It represents superposition of $\mathrm{FeO}(111) / \mathrm{Pt}(111)$ and $\mathrm{Fe}_{3} \mathrm{O}_{4}(111)$

LEED patterns shown in Fig. 5.3b and c. Corresponding reciprocal unit cells are indicated. From [234].

Figure $5.19 \mathrm{~b}$ shows the film after additional evaporation-oxidation cycles. No increase in the number of $\mathrm{Fe}_{3} \mathrm{O}_{4}$ islands is observed, even after inspection of many images. Instead, the $\mathrm{Fe}_{3} \mathrm{O}_{4}$ islands have increased in size. Now they are 40-50 $\AA$ high and form almost perfect triangles with side lengths of 6000 to $7000 \AA$. Triangular shaped islands rotated by $180^{\circ}$ against each other are found at the surface as can be seen on the right border of the image in Fig. 5.19b. The side edges of the islands run along the $[\overline{1} 10]$ and $[01 \overline{1}]$ directions of the Pt substrate and the $\mathrm{Fe}_{3} \mathrm{O}_{4}(111)$ surface, as is known from LEED and from high-resolution STM images. Some islands have coalesced already at this stage. The island surfaces exhibit high step densities with terraces 100-300 $\AA$ wide. The step height is mostly 4.8 $\AA$, which corresponds to the distance between equivalent (111) surface terminations of $\mathrm{Fe}_{3} \mathrm{O}_{4}$. As the growth proceeds, new small islands with hexagonal shapes start to grow between and on top of the large triangular $\mathrm{Fe}_{3} \mathrm{O}_{4}$ islands (Fig. 5.19c). These new islands also increase in size and eventually coalesce, until the whole substrate surface is covered by a thick $\mathrm{Fe}_{3} \mathrm{O}_{4}$ film (Fig. 5.19d). From the deposited amount of Fe and the island heights, we estimate this film to be at least $100 \AA$ thick. It can be grown thicker by further Fe deposition-oxidation cycles. The $\mathrm{FeO}(111)$ satellite LEED pattern has vanished and the $\mathrm{Fe}_{3} \mathrm{O}_{4}(111)$ LEED pattern (Fig. 5.3c) is observed at this point. 


\subsection{2. $\mathrm{Fe}_{3} \mathrm{O}_{4}$ growth at $\mathrm{T}=1000 \mathrm{~K}$}

The $1 \times 1 \mu \mathrm{m}^{2}$ images in Fig. 5.20 display the Fe-oxide growth for an oxidation temperature of 1000 $\mathrm{K}$, after a complete $\mathrm{FeO}(111)$ monolayer has grown onto the $\mathrm{Pt}(111)$ surface. For all growth stages the LEED patterns of Fig. 5.19e are observed, indicating the formation of $\mathrm{Fe}_{3} \mathrm{O}_{4}(111)$ islands. The islands form crystallites with straight edges and triangular or hexagonal shapes, depending on the growth stage and exact growth conditions. They are atomically flat with very few steps $4.8 \AA$ high, which again indicates the existence of one $\mathrm{Fe}_{3} \mathrm{O}_{4}(111)$ surface termination. The island edges run along the [1 10$]$ and $[01 \overline{1}]$ directions of $\mathrm{Fe}_{3} \mathrm{O}_{4}$. Due to the finite aspect ratio of the STM tip, it was not possible to determine the exact slope of the island side facets. Slopes of less than $70^{\circ}$ can be ruled, out because this slope was measured for neighboring facets of hexagonal islands and for facets of triangular islands. A slope of $90^{\circ}$ corresponds to a vertical side facet.
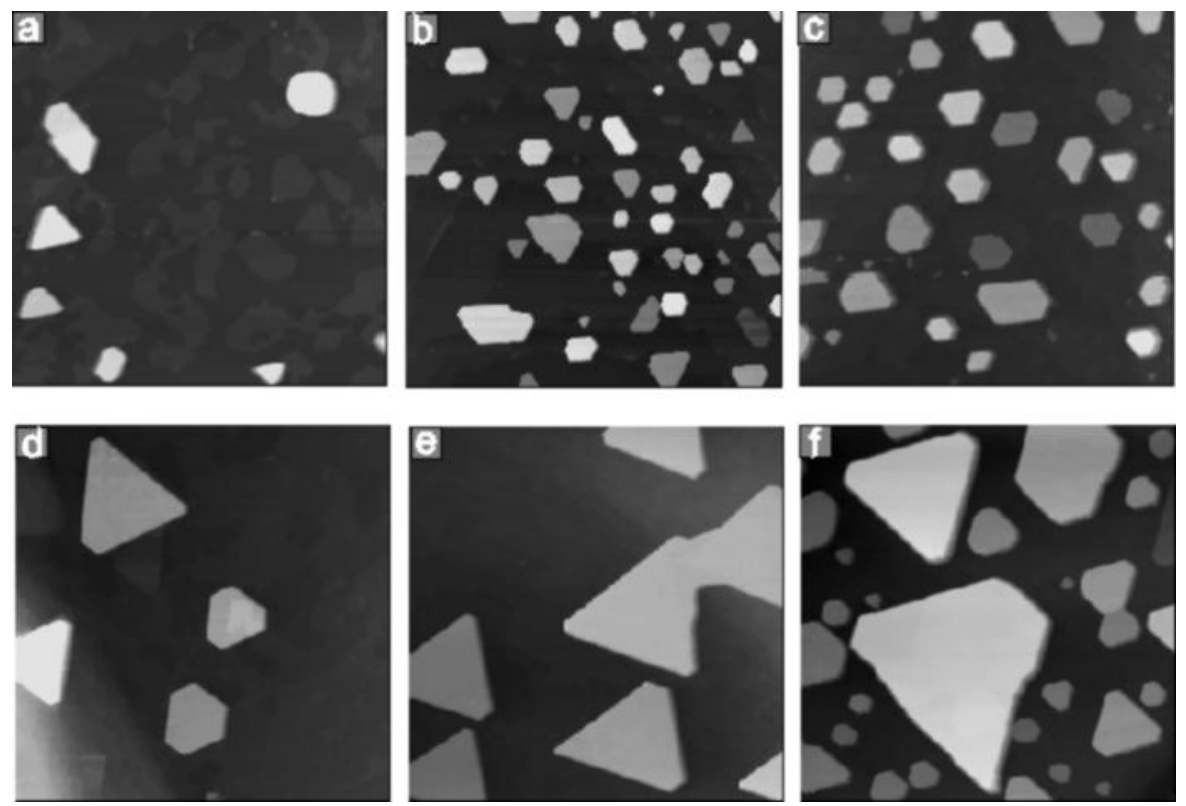

Fig. 5.20. $1 \times 1 \mu \mathrm{m}^{2} \mathrm{STM}$ images of consecutive $\mathrm{Fe}_{3} \mathrm{O}_{4}$ island growth stages for oxidation temperature of 1000 K. In (a)-(c) Fe deposition per cycle was low (same as for films in Fig. 5.19). Islands are 25-70 Å high. In (d)-(f) Fe deposition rate was 10 times larger. Island heights are 35-60 $\AA$ in (d), 40-70 $\AA$ in (e) and 25$100 \AA$ in (f). From [234].

In Fig. 5.20a-c, the amount of Fe deposited in each cycle is about the same as for the films grown at $\mathrm{T}=870 \mathrm{~K}$, presented in the previous section. Initially, Fig. 5.20a, hexagonal and some triangular shaped islands with heights between 25 and $60 \AA$ and lateral dimensions around $1000 \AA$ have formed. Triangular islands rotated by $180^{\circ}$ can also be seen. After additional deposition-oxidation cycles (Fig. $5.20 \mathrm{~b}$ ), the number of islands has increased, but not their sizes. Only the island heights have increased slightly, now they range from 25 to $70 \AA$. More hexagonal islands have formed. The number and size of islands does not change upon further Fe deposition-oxidation cycles, as can be seen in Fig. 5.20c. No coalescence of island is observed and no closed $\mathrm{Fe}_{3} \mathrm{O}_{4}$ film can be obtained.

Figure 5.20d-f displays the $\mathrm{Fe}_{3} \mathrm{O}_{4}$ island growth at $1000 \mathrm{~K}$ oxidation temperature with the amount of Fe deposited before each oxidation cycle increased by a factor of ten, when compared to Fig. 5.20ac. Initially (Fig. 5.20d), two small islands with hexagonal shape and two large islands with triangular shape rotated by $180^{\circ}$ against each other can be seen. The island heights range from 35 to $60 \AA$. After further growth (Fig. 5.20e), the islands have grown larger both laterally and in height. Now they are 40-70 A high. All islands are triangular. Upon further deposition-oxidation cycles (Fig. 5.20f), some islands have coalesced to form the largest one in this image, and new small islands start to grow between the large islands. The small islands are 25-60 $\AA$ high, the large ones 80-100 $\AA$. Although this growth behavior is similar to that observed in Fig. 5.19 for $870 \mathrm{~K}$ oxidation temperature, no 
completely closed $\mathrm{Fe}_{3} \mathrm{O}_{4}$ films can be obtained at $1000 \mathrm{~K}$. However, at slightly lower temperatures $(920 \mathrm{~K})$, closed films can be grown, as is shown in the next section.

\subsubsection{Morphology of $\mathrm{Fe}_{3} \mathrm{O}_{4}(111)$ and $\alpha-\mathrm{Fe}_{2} \mathrm{O}_{3}(0001)$ multilayer films}

Figure 5.21 shows $1 \times 1 \mu \mathrm{m}^{2}$ images of three $\mathrm{Fe}_{3} \mathrm{O}_{4}(111)$ (a-c) films and an $\alpha-\mathrm{Fe}_{2} \mathrm{O}_{3}(0001)$ film (d) together with cross-section scans along the white lines in the images. The film in Fig. 5.21a was grown at $\mathrm{T}=870 \mathrm{~K}$ oxidation temperature. Large (2000-4000 $\AA$ ) and smaller islands have coalesced, resulting in a film that is at least $100 \AA$ thick. The mesoscopic surface roughness is about $40 \AA$ on the length scale of $1 \mu \mathrm{m}$ and a high step density has formed with terrace widths between 50 and $500 \AA$. Most steps are about $4.8 \AA$ high, indicating the dominance of one $\mathrm{Fe}_{3} \mathrm{O}_{4}(111)$ surface termination. After annealing this film for $15 \mathrm{~min}$ at $1000 \mathrm{~K}$ in $10^{-6} \mathrm{mbar}_{2}$ (Fig. 5.21b), the surface step density has decreased considerably. Atomically flat terraces, more than $1000 \AA$ wide have formed, separated by steps $4.8 \AA$ (or multiples thereof) high. The LEED spots have become sharper and the background intensity has decreased. Screw dislocations can be seen; two are marked by arrows in Fig 5.21b. The cross-section shows, however, that the mesoscopic surface roughness is not significantly reduced compared to the film in Fig. 5.21a.
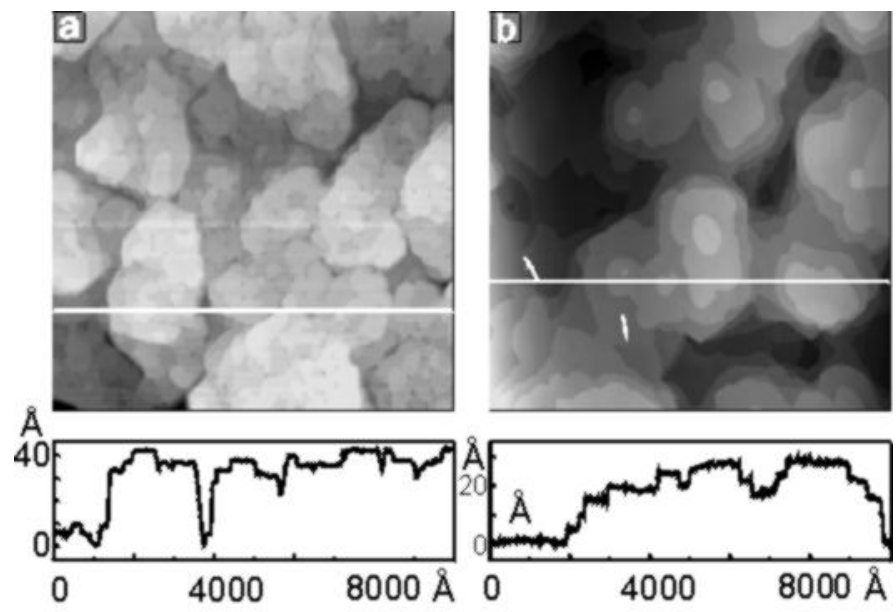

Fig. 5.21. $1 \times 1 \mu \mathrm{m}^{2}$ STM images and crosssection scans of closed Fe-oxide films at least $100 \AA$ thick. $\mathrm{Fe}_{3} \mathrm{O}_{4}(111)$ film in (a) was grown at $T=870 \mathrm{~K}$. Post-annealing of this film to $T=1000$ $\mathrm{K}$ creates film shown in (b); white arrows mark screw dislocations. $\mathrm{Fe}_{3} \mathrm{O}_{4}(111)$ film in (c) was grown at $T=920 \mathrm{~K}$. d) shows $\alpha-\mathrm{Fe}_{2} \mathrm{O}_{3}(0001)$ film obtained by oxidizing film in b) at $T=1000$ $\mathrm{K}$ in $p\left(\mathrm{O}_{2}\right)=10^{-1}$ mbar. From [234].

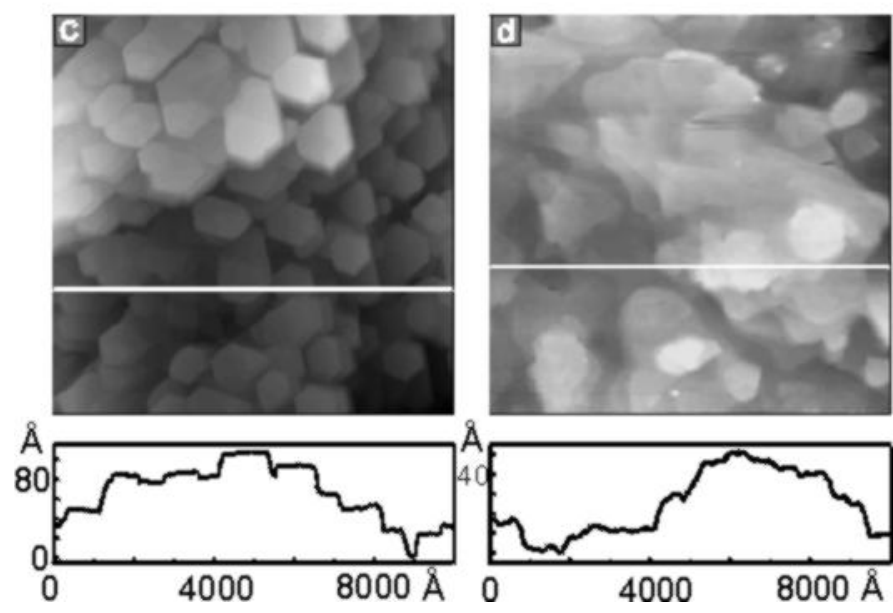

For oxidation temperatures of $1000 \mathrm{~K}$, no closed films had been obtained, but at $920 \mathrm{~K}$ they are closed (Fig. 5.21c). Their morphology differs significantly from the morphologies of the films in Fig. 5.21 $\mathrm{a}$ and $\mathrm{b}$. The entire substrate is covered by mostly hexagonal crystallites 1000-2000 $\AA$ in diameter. They form a closed $\mathrm{Fe}_{3} \mathrm{O}_{4}$ film with a mesoscopic surface roughness of about $100 \AA$ on a length scale 
of $1 \mu \mathrm{m}$. The crystallite surfaces are atomically flat with $1000 \AA$ wide terraces and steps $4.8 \AA$ (or multiples thereof) high, again indicating the existence of one $\mathrm{Fe}_{3} \mathrm{O}_{4}(111)$ surface termination. The terrace widths decrease rapidly towards the crystallite edges, where step bunching occurs, which is not resolved in the cross-section of Fig. 5.21c. The morphology of these films does not change upon post annealing for $15 \mathrm{~min}$ at $\mathrm{T}=1000 \mathrm{~K}$ in $10^{-6} \mathrm{mbar} \mathrm{O}_{2}$. Compared to the lower oxidation temperature of $870 \mathrm{~K}$, more Fe deposition-oxidation cycles were necessary at $920 \mathrm{~K}$ to obtain closed films, indicating that coalescence startsat a larger island heights.

The $\mathrm{Fe}_{3} \mathrm{O}_{4}(111)$ film in Fig. 5.21b was transformed into an $\alpha-\mathrm{Fe}_{2} \mathrm{O}_{3}(0001)$ film (Fig. 5.21d), by a high pressure oxidation in $10^{-1}$ mbar at $1000 \mathrm{~K}$. After oxidation, the sample was cooled down in $\mathrm{O}_{2}$, in order to quench the structure formed under the high $\mathrm{O}_{2}$ pressure. The $\mathrm{Fe}_{3} \mathrm{O}_{4}(111)$ LEED pattern (Fig. 5.3c), has vanished and the LEED pattern (Fig. 5.3d), was obtained, which corresponds to an unreconstructed (0001) surface termination of $\alpha-\mathrm{Fe}_{2} \mathrm{O}_{3}$ (see Section 5.2A). The hexagonal O (0001) planes are aligned to those of the former $\mathrm{Fe}_{3} \mathrm{O}_{4}(111)$ film and, thus, to the $\mathrm{Pt}(111)$ surface lattice. The mesoscopic surface roughness in Fig. 5.21d is almost unchanged and is about $50 \AA$ on a length scale of $1 \mu \mathrm{m}$. Although the LEED pattern of this film was of good quality, only few atomically flat terraces were observed. Better surface order was obtained after oxidation at $1120 \mathrm{~K}$, where atomically flat terraces upto $500 \AA$ wide developed. Domains exposing two different terminations of $\alpha-\mathrm{Fe}_{2} \mathrm{O}_{3}(0001)$ are observed on these terraces (see Section 5.6). Equivalent terminations are separated by steps $2.3 \AA$ (or multiples thereof) high.

\subsection{4. $\mathrm{Fe}_{3} \mathrm{O}_{4}$-Pt interface structure}

From the LEED pattern of the $\mathrm{Fe}_{3} \mathrm{O}_{4}$ island films in Fig. 5.19e, it follows that the hexagonal $\mathrm{O}$ (111) planes in the $\mathrm{Fe}_{3} \mathrm{O}_{4}$ magnetite structure are aligned to those of the hexagonal $\mathrm{Pt}(111)$ surface lattice and to those of the first $\mathrm{FeO}(111)$ layers, as displayed (Fig. 5.3). The high-resolution LEED measurement shown in Fig. 5.12 for $\theta_{\mathrm{FeO}}=2.2 \mathrm{ML}$ reveal the $\mathrm{Fe}_{3} \mathrm{O}_{4}$ bulk lattice constant for the islands. The question whether the first $\mathrm{FeO}$ layers under the islands retain their structure or transform into $\mathrm{Fe}_{3} \mathrm{O}_{4}$ was resolved by transmission electron microscopy (TEM) in combination with image simulation techniques [268]. Figure 5.22 is a high-resolution TEM image of the $\mathrm{Fe}_{3} \mathrm{O}_{4} / \mathrm{Pt}$ interface. The simulated image shows that the $\mathrm{Fe}_{3} \mathrm{O}_{4}$ lattice continues right through, until the interface without an $\mathrm{FeO}$ layer, which would have a different lattice constant. However, the lattice mismatch between $\mathrm{Pt}$ and $\mathrm{Fe}_{3} \mathrm{O}_{4}$ induces local variations of the lattice constant at the interface of upto $\pm 2 \%$, leading to periodic strain fields. According to the crystal structure of $\mathrm{Fe}_{3} \mathrm{O}_{4}$ (Fig. 5.2b), the first Fe layer on Pt may arrange as a Kagomé layer or as mix-trigonal layers, which would result in a different height of the following first O layer and, thus, induce antiphase boundaries (APB's) in the film. Such APB's, with the expected shift of $1.86 \AA$ in [111] direction of both domain types with respect to each other, were actually observed (Fig. 5.23), which shows that both $\mathrm{Fe}_{3} \mathrm{O}_{4}$ terminations at the interface are possible.

In $\mathrm{FeO}$, both the $\mathrm{Fe}$ layers and the $\mathrm{O}$ layers are fully close packed. $\mathrm{In}_{\mathrm{Fe}} \mathrm{O}_{4}$, however, the $\mathrm{Fe}$ layers (both the Kagomé or the three mix-trigonal layers) contain only 3 atoms per $4 \mathrm{O}$ atoms. Even if we assume the most expanded structure 4 for the initial $\mathrm{FeO}$ layer, about $13 \%$ of the first $\mathrm{Fe}$ layer has to be removed during its transformation into $\mathrm{Fe}_{3} \mathrm{O}_{4}$. Obviously, the mobility of $\mathrm{Fe}$ during the oxidation treatment at $870-1000 \mathrm{~K}$ is high enough to incorporate the first layer $\mathrm{Fe}$ into the forming $\mathrm{Fe}_{3} \mathrm{O}_{4}$ islands. The initial $\mathrm{FeO}$ layer is, thus, not a stable enough entity to maintain its nature below the growing $\mathrm{Fe}_{3} \mathrm{O}_{4}$ phase. 

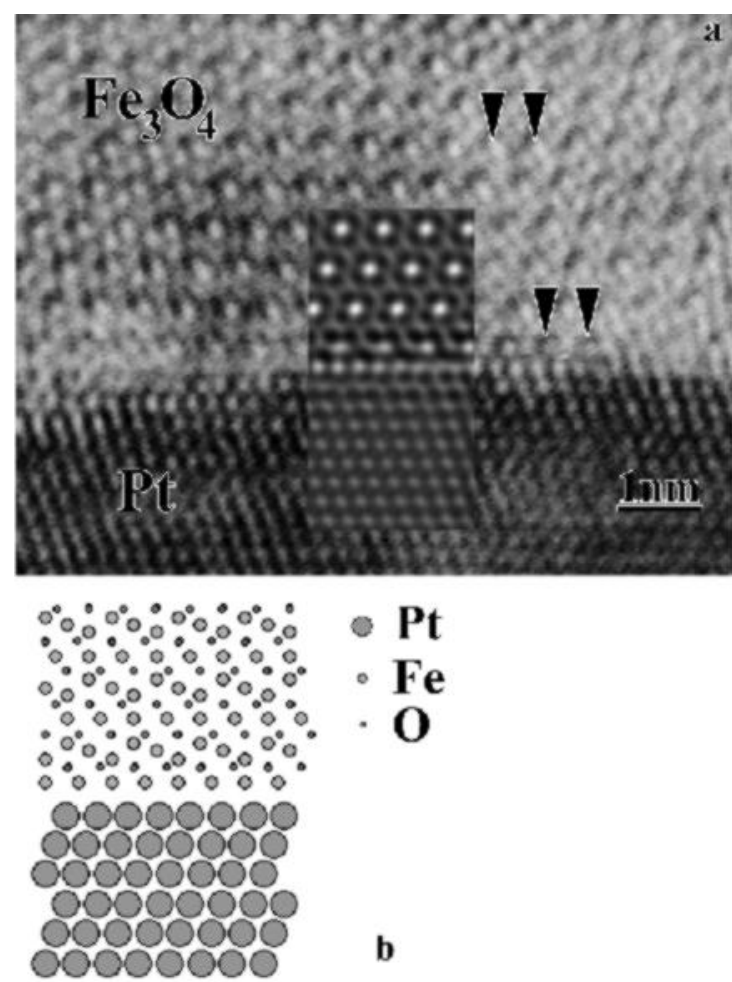

Fig. 5.22.(a) High-resolution TEM image along [1 $1 \overline{1} 0]$ direction of $\mathrm{Fe}_{3} \mathrm{O}_{4} / \mathrm{Pt}$ interface with simulated image as inset. Spots marked by arrows near interface are contracted by $2 \%$ compared to corresponding spots marked in bulk. (b) Atomic model corresponding to simulated image of interface. After [268].
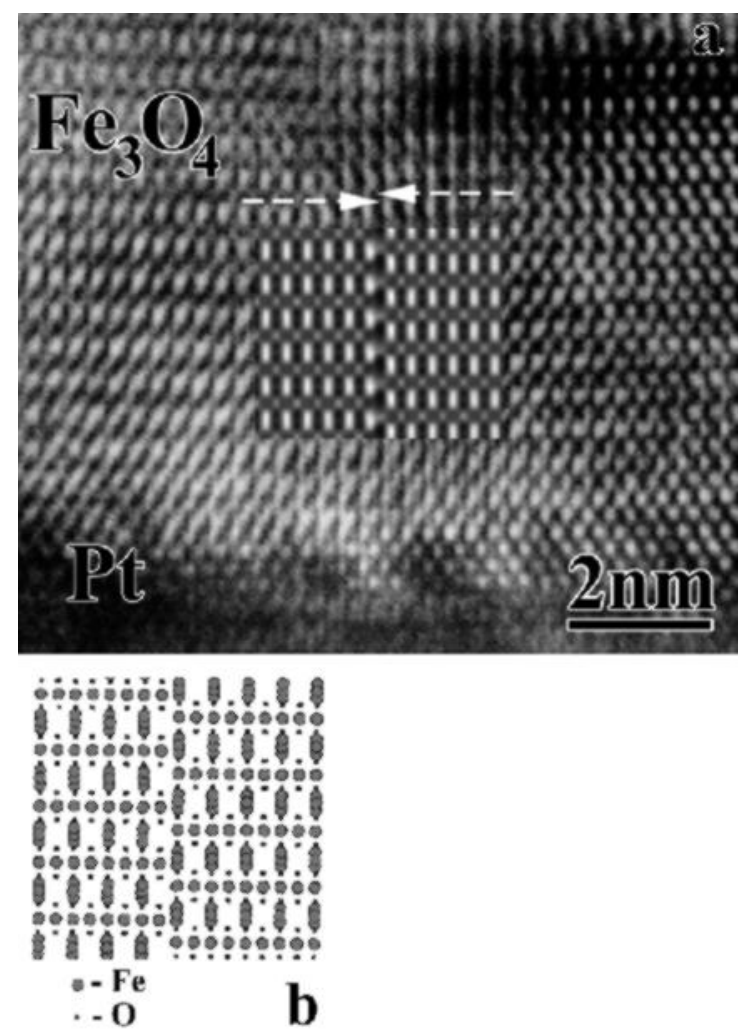

Fig. 5.23. (a) High-resolution TEM image along $[11 \overline{2}]$ direction of antiphase boundary between two domains in $\mathrm{Fe}_{3} \mathrm{O}_{4}$ film with shift of $1.86 \AA$ along [111] direction. Simulated image is shown as inset. (b) Projection of corresponding atomic model. After[268].

\subsubsection{Discussion}

\section{(i) $\mathrm{Fe}_{3} \mathrm{O}_{4}$ island formation}

The higher the growth temperature, the thinner is the $\mathrm{FeO}$ film onto which island formation starts: for $T=1000 \mathrm{~K}$ at $1 \mathrm{ML} \mathrm{FeO}$ and for $T=870 \mathrm{~K}$ at around $2 \mathrm{ML} \mathrm{FeO}$. Due to the higher mobility at elevated temperatures, the less stable second and third $\mathrm{FeO}(111)$ layers can rearrange more easily to form $\mathrm{Fe}_{3} \mathrm{O}_{4}(111)$ islands. Figure 5.24 shows the initial island formation for an intermediate oxidation temperature of $T=920 \mathrm{~K}$. Island growth starts when the $\mathrm{FeO}$ coverage is about 1.6 ML. The shape and size of the bright third layer island, about $1200 \times 500 \AA^{2}$ in size in the upper part of Fig. 5.24a, resembles the $\mathrm{Fe}_{3} \mathrm{O}_{4}(111)$ islands in Fig. 5.20a-c. Two regions with different surface structures are found on its surface. In high-resolution STM images, the brighter surface regions exhibit $1.5 \AA$ high cluster-like features without long-range order, whereas the darker regions are well-ordered. The vertical height difference between the second $\mathrm{FeO}$ layers and the ordered surface regions on the island is $2.5 \AA$, which corresponds to the distance between consecutive Fe-O (111) bilayers in the bulk FeO structure. A $100 \times 100 \AA^{2}$ atomic resolution STM image of the ordered region is shown in Fig. 5.24b. It exhibits an unreconstructed $\mathrm{FeO}(111)-(1 \times 1)$ surface forming the non-rotated coincidence structure 4 discussed in Section 5.3.3. Several randomly distributed defects exist. Missing corrugation maxima are in registry with the corrugation maxima on the defect-free parts. Therefore, they are interpreted as $\mathrm{O}$ vacancies. Since the surface structure and morphology of the bright island in Fig. 5.24a is clearly 
different from the third layer $\mathrm{FeO}(111)$ islands grown at $870 \mathrm{~K}$ (Fig. 5.10f and 5.11d), this island represents the initial growth stage of an $\mathrm{Fe}_{3} \mathrm{O}_{4}(111)$ island.

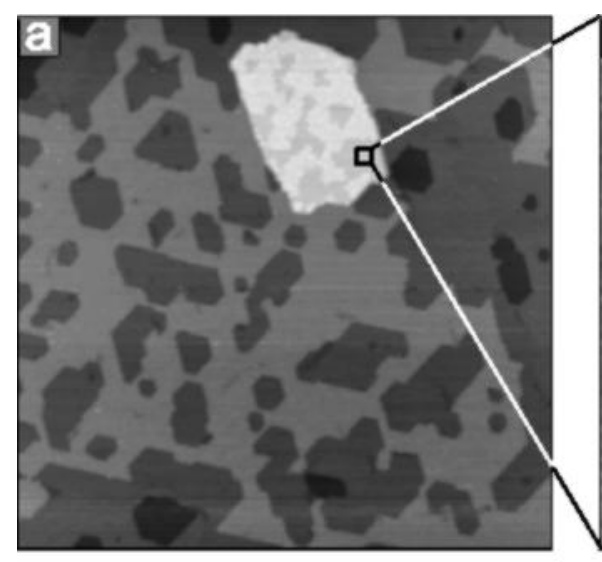

$1,6 \mathrm{ML}$ FeO, $3500 \times 3500 \AA^{2}$

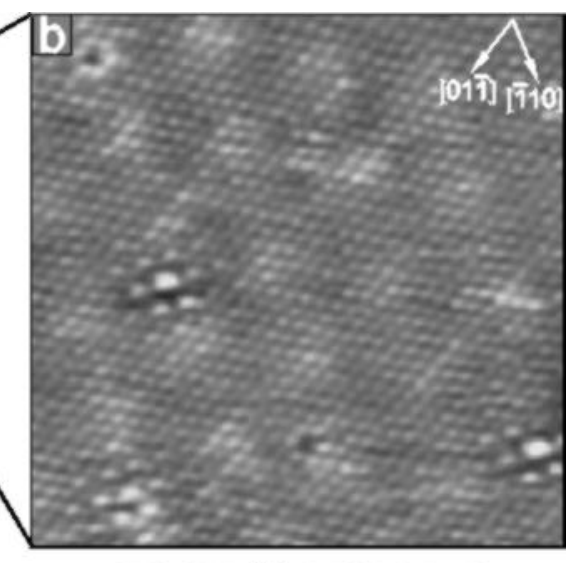

$100 \times 100 A^{2} ; 0.3 V, 1.0 \mathrm{nA}$
Fig. 5.24. (a) Large scale STM image of 1.6 ML thick $\mathrm{FeO}(111)$ film prepared at $920 \mathrm{~K}$ oxidation temperature. Bright third layer island $1200 \times 500 \AA^{2}$ in size represents initial growth stage of $\mathrm{Fe}_{3} \mathrm{O} 4(111)$ island. (b) Unreconstructed $\mathrm{FeO}(111)-(1 \times 1)$ surface structure with $(8 \times 8)$ coincidence structure 4 on top of island [269].

For oxide-on-oxide growth, different cases were discussed. A layer-by-layer growth was observed in case of small lattice mismatch, e.g., for $\mathrm{Fe}_{3} \mathrm{O}_{4}(001)$ on $\mathrm{MgO}(001)$ [171] and for $\mathrm{Fe}_{3} \mathrm{O}_{4}(110)$ on $\mathrm{MgO}(110)$ (0.3\% mismatch) [167], whereas island growth was observed for large mismatch, e.g., for $\mathrm{Fe}_{3} \mathrm{O}_{4}(111)$ on $\mathrm{Al}_{2} \mathrm{O}_{3}(0001)$ [166, 168] (8.4\% mismatch). Depending on the island size and on the interface/surface energies, dislocated unstrained or coherently strained islands can form on top of the first wetting layers [265]. If the large activation energy for the formation of dislocations at the interface can be overcome, dislocated islands without strain grow [270]. The growth of unstrained islands can be described by an Ostwald ripening mechanism, which was formulated theoretically by Lifschitz [271] and Wagner [272] for crystal growth in supersaturated solutions and extended later to epitaxial island growth on substrates by Chakraverty [273]. Since the the underlying $\mathrm{FeO}$ layer dissolves into the $\mathrm{Fe}_{3} \mathrm{O}_{4}$ islands, in our case, the island formation cannot be explained by these mechanisms. It seems more likely that homogeneous nucleation of the islands occurs and that the strain imposed on the FeO layer by the growing island facilitates the out diffusion of $\mathrm{Fe}$.

According to the $\mathrm{Fe}-\mathrm{O}_{2}$ bulk phase diagram depicted in Fig. 5.1, the formation of $\alpha-\mathrm{Fe}_{2} \mathrm{O}_{3}$ is expected in most of the range where $\mathrm{Fe}_{3} \mathrm{O}_{4}$ is formed $\left(p\left(\mathrm{O}_{2}\right)=10^{-6} \mathrm{mbar}, T=870-1000 \mathrm{~K}\right)$, which is due to the oxidation kinetics. The oxidation of $\mathrm{Fe}_{3} \mathrm{O}_{4}$ to $\alpha-\mathrm{Fe}_{2} \mathrm{O}_{3}$ is obviously a slow process. After annealing $\mathrm{Fe}_{3} \mathrm{O}_{4}(111)$ multilayer films for several hours at our $\mathrm{Fe}_{3} \mathrm{O}_{4}$ growth conditions, a film consisting of both $\mathrm{Fe}_{3} \mathrm{O}_{4}$ and $\alpha-\mathrm{Fe}_{2} \mathrm{O}_{3}$ phases was detected by LEED. That the oxidation kinetics determines the Fe-oxide phase formed during epitaxial growth was also observed by Kim et al. [170], who demonstrated that the selective growth of $\mathrm{Fe}_{3} \mathrm{O}_{4}$ and $\alpha-\mathrm{Fe}_{2} \mathrm{O}_{3}$ films depends on the growth rate and oxygen pressure, during oxygen-plasma assisted molecular beam epitaxy. High growth rates and low oxygen pressures were required to obtain $\mathrm{Fe}_{3} \mathrm{O}_{4}$, while low growth rates and high oxygen pressures were needed to obtain $\alpha-\mathrm{Fe}_{2} \mathrm{O}_{3}$.

\section{(ii) $\mathrm{Fe}_{3} \mathrm{O}_{4}$ island facets}

Large $\mathrm{Fe}_{3} \mathrm{O}_{4}$ islands, mostly with triangular basal planes and small islands with mostly hexagonal basal planes, were observed as schematically indicated in Fig. 5.25. The STM measurements reveal contact angles $\alpha$ between the substrate surface and the facet planes to be at least $70^{\circ}$. $\{100\}$ planes as island facets can be ruled out, because they have contact angles of $55^{\circ}$ and $125^{\circ}$. The most stable surfaces of magnetite are the $\{111\}$ and $\{110\}$ planes, leading to octahedral and rhombodecahedral 
macroscopic crystal forms [224]. As known from atomic resolution STM images measured on the island surfaces, the island edges run parallel to the atomic rows on the $\mathrm{Fe}_{3} \mathrm{O}_{4}(111)$ surface, i.e., along the $[\overline{1} 10]$ and $[01 \overline{1}]$ directions indicated in Fig. 5.3c. $\{110\}$ planes that are compatible with these side edges are the (110) and (110) planes and those obtained by the p3m1 symmetry of the $\mathrm{Fe}_{3} \mathrm{O}_{4}(111)$ surface. However, they can also be ruled out since they have contact angles of $35^{\circ}$ and $145^{\circ}$, respectively. Therefore, we attribute three of the six island facets to (111) planes and symmetrically equivalent ones, as indicated in Fig. 5.25, because they form an angle $\alpha$ of $70.5^{\circ}$ to the substrate surface and island edges along the atomic rows as observed. The next stable low-index surface planes of magnetite are the $\{112\}$ planes, and we attribute $(2 \overline{1} \overline{1})$-type planes to the other three facets of the hexagonal islands. These facets form a contact angle of $90^{\circ}$ and also island edges as observed.
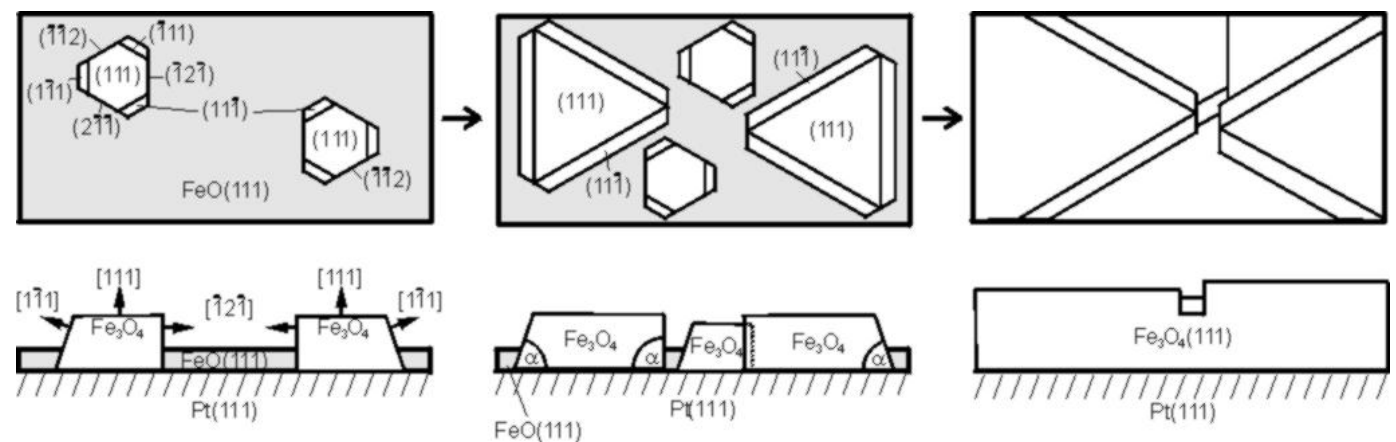

Fig. 5.25.

Schematic representation of Stranski-Krastanov growth for Feoxides on $\mathrm{Pt}(111)$. Island surface and facet plane orientations are indicated.

\section{(iii) $\mathrm{Fe}_{3} \mathrm{O}_{4}$ island growth mechanism}

We do not observe any influence of defects on the $\operatorname{Pt}(111)$ substrate on the island formation. Therefore, we assume a homogeneous nucleation mechanism, which takes place during each oxidation cycle following the Fe deposition at room temperature. A super-saturated solution of Fe or an Fe-oxide precursor is generated on top of the $\mathrm{FeO}(111)$ films, resulting in a uniform nucleation probability. Three different island growth scenarios were observed: Regime 1 - oxidation temperature $870 \mathrm{~K}$ (Fig. 5.19); regime 2 - oxidation temperature $1000 \mathrm{~K}$ (Fig. 5.20a-c); regime 3 - oxidation temperature also $1000 \mathrm{~K}$, but the amount of Fe deposited in each growth cycle was one order of magnitude larger (Fig. 5.20d-f). In all regimes, the islands grow much faster laterally than vertically. The aspect ratio of the islands (height/basal plane diameter) increases with increasing oxidation temperature, indicating an additional activation energy for the upward diffusion of Fe-oxide on the island facets. At the higher oxidation temperature in regimes 2 and 3, there is a higher Fe mobility, which leads to smoother island surfaces and straighter island edges in comparison to regime 1.

Most small islands with diameters below $2000 \AA$ are hexagonal, all larger islands are triangular. If a crystal is in equilibrium with its vapor phase, the total surface free energy is minimized and the equilibrium crystal shape can be obtained by the Wulff construction, provided the relevant surface free energies are known [274, 275]. The hexagonal islands might be close to the equilibrium shape of such magnetite crystallites. However, if a crystal grows from a super-saturated solution, the growth shape of the crystal may deviate from its equilibrium shape, because the growth velocity perpendicular to a crystal plane increases with the corresponding surface free energy. Here, the fast growing $(2 \overline{1} \overline{1})$ planes eventually disappear and the slow growing stable ( $\overline{1} 11)$ surface planes remain, leading to large triangular islands that expose only (111) facets, as indicated in Fig. 5.25 [262]. The formation of triangular shaped islands rotated by $180^{\circ}$ indicates the existence of two in-plane orientations of $\mathrm{Fe}_{3} \mathrm{O}_{4}(111)$ related by an $180^{\circ}$ rotation about the [111] axes. This agrees with LEED measurements, 
which sometimes revealed a sixfold rotational symmetry of the LEED pattern. This symmetry can only be explained by $180^{\circ}$ domain orientations being simultaneously present at the surface, since the $\mathrm{Fe}_{3} \mathrm{O}_{4}(111)$ surface has a threefold rotational symmetry. Such rotational twinning has also been observed for systems, such as $\mathrm{Pt}(111)$ on $\alpha-\mathrm{Al}_{2} \mathrm{O}_{3}(0001)$ [276] and $\mathrm{Fe}_{3} \mathrm{O}_{4}(111)$ on $\alpha-\mathrm{Al}_{2} \mathrm{O}_{3}(0001)$ [168]. It is a natural growth mode for (111) films of fcc crystals and is caused by the random occurrence of $\mathrm{ABCABC}$ and $\mathrm{ACBACB}$ stacking sequences, which presumably has a minor effect on the interfacial energy or nucleation probability.

In all three regimes, similar numbers of islands are formed at the beginning, as expected for a homogeneous nucleation mechanism, which takes place in the oxide precursor solution formed by the Fe deposited onto $\mathrm{FeO}(111)$. During further growth cycles, the islands formed in regime 1 and 3 increase in size, but their number remains almost constant. This is due to an Ostwald ripening mechanism [271-273]: During each oxidation cycle, new small islands nucleate from the supersaturated oxide precursor solution between the larger islands that have already formed. As a result of the Gibbs-Thomson relation [272], the small islands have a larger solubility in the surrounding precursor solution, which results in material transport from the small to the large islands leading to dissolution of the small islands. In this way the large islands grow at the expense of the small islands, resulting in an increase in size, but not in the number of the islands. When the islands reach lateral sizes of 3000-5000 $\AA$ and triangular shapes, new small islands start to form between them. Since triangular shaped islands only expose (111) facets with a small growth velocity, the material transport to them slows down. As a result, small islands that nucleate during the following oxidation cycles, do not dissolve anymore within the oxidation time. The small islands in Fig. 5.20f exhibit hexagonal, or even almost round shapes, which indicates that dissolution from their edges has started, as a result of the ripening process.

In regime 2 , the sizes of the initially formed islands do not increase, upon further growth cycles. The number of islands increases at the beginning and remains constant. During each oxidation cycle, the oxide precursor solution is removed from the regions between the islands by oxide island growth, but at $T=1000 \mathrm{~K}, \mathrm{Fe}$ is also lost by diffusion into the Pt substrate and by desorption into the gas-phase as observed by ISS [160] and mass spectrometry [235]. As the total surface area of all islands also increases the loss of Fe-oxide by evaporation becomes significant. Since only small amounts of $\mathrm{Fe}$ were deposited, the precursor solution is consumed quickly. The resulting slow growth is balanced by the Fe-oxide loss, due to evaporation from the islands. When the situation in Fig. 5.20b or c is reached, no new islands can grow, since all islands that nucleate dissolve again, which results in the observed constant number and size of islands. In regime 3 , with an oxidation temperature of $1000 \mathrm{~K}$, the material losses are the same as in regime 2, but due to the much larger amount of deposited Fe the precursor solution is not consumed as quickly, so that ripening can occur. In Fig. 5.20c, the total amount of deposited $\mathrm{Fe}$ and the total oxidation time were higher than in Fig. 5.20d, although less ripening took place. This indicates that the material transport during ripening cannot simply be explained by surface diffusion, but needs the existence of the precursor solution.

\section{(iv) $\mathrm{Fe}_{3} \mathrm{O}_{4}$ and $\mathrm{\alpha}-\mathrm{Fe}_{2} \mathrm{O}_{3}$ multilayer films}

Considering the lateral and vertical $\mathrm{Fe}_{3} \mathrm{O}_{4}$ island dimensions of 3000-5000 $\AA$ and $100 \AA$, respectively, their morphology is described by flat platelets rather than by columns. For oxidation temperatures between $870 \mathrm{~K}$ and $920 \mathrm{~K}$, these islands eventually coalesce and form closed films at least $100 \AA$ thick, which can be transformed into $\alpha-\mathrm{Fe}_{2} \mathrm{O}_{3}(0001)$ films by a high pressure oxidation. The orientations of all three oxide phases with respect to the Pt substrate are depicted in Fig. 5.3. The epitaxial relationships with respect to the $\mathrm{Pt}$ substrate are $\mathrm{Fe}_{3} \mathrm{O}_{4}[\overline{1} 10] \| \mathrm{Pt}[\overline{1} 10]$ and $\mathrm{Fe}_{2} \mathrm{O}_{3}[1 \overline{1} 00] \| \mathrm{Pt}[\overline{1} 10]$ and correspondingly for the $180^{\circ}$ twinning domains. 
The dominance of one single surface termination separated by steps $4.8 \AA$ (or multiples thereof) high is observed on all $\mathrm{Fe}_{3} \mathrm{O}_{4}(111)$ films, and is discussed in more detail in Section 5.5. The step densities on the films grown at $870 \mathrm{~K}$ (Fig. 5.21a) decrease after a post-annealing treatment to $1000 \mathrm{~K}$ in $10^{-6} \mathrm{mbar}_{2}$, whereas no changes occur after post-annealing the films grown at $920 \mathrm{~K}$ (Fig $5.21 \mathrm{c}$ ), which indicates that a stable $\mathrm{Fe}_{3} \mathrm{O}_{4}$ film morphology is established around $920 \mathrm{~K}$. The mesoscopic surface roughness of the $\mathrm{Fe}_{3} \mathrm{O}_{4}(111)$ films grown at 870 is three times lower than at $920 \mathrm{~K}$ (Fig. 5.21b, c) and the atomic-step densities on these two films differ in a similar way. The mesoscopic surface roughness of the $\alpha-\mathrm{Fe}_{2} \mathrm{O}_{3}(0001)$ films is similar to that of the $\mathrm{Fe}_{3} \mathrm{O}_{4}(111)$ films from which it was prepared by high pressure oxidation, but the atomic-step density is considerably higher. This high step density can be partly reduced by increasing the temperature during the high pressure oxidation, which shows that the mesoscopic and atomic surface roughness can be controlled, within a certain range, by choosing the proper film growth and preparation conditions.

\subsection{Surface structures of $\mathrm{Fe}_{3} \mathrm{O}_{4}(111)$ films}

\subsubsection{Structures formed below $\mathrm{T}=1000 \mathrm{~K}$}

Different surface structures coexist on $\mathrm{Fe}_{3} \mathrm{O}_{4}(111)$ multilayer films prepared at oxidation temperatures between 870 and $920 \mathrm{~K}$ [277]. They all display hexagonal surface lattices in the atomic resolution STM images, which have unit cells with a lattice constant of $6 \AA$ that are oriented along the [110] and [01 $\overline{1}$ ] directions of the $\mathrm{Fe}_{3} \mathrm{O}_{4}(111)$ surface. This is in line with the $\mathrm{Fe}_{3} \mathrm{O}_{4}(111)$ LEED pattern observed on these films (Fig. 5.3c). About $90 \%$ of the surface is covered by regions denoted $\chi$ in Fig. 5.26 with one protusion within the $6 \AA$ hexagonal unit cell. The rest of the surface is covered by islands and domains with different atomic structures that apparently are located somewhat deeper than the surrounding $\chi$ regions. An example is shown in a large-scale image in Fig.5.26a. Three different regions denoted $\alpha, \beta$ and $\gamma$ exist within this island, as shown in Fig. 5.26b. They form triangular and hexagonal patches arranged to form a superlattice with a $50 \AA$ periodicity along the $[\overline{1} 10]$ and [01 $\overline{1}]$ directions on the $\mathrm{Fe}_{3} \mathrm{O}_{4}(111)$ surface. The $\beta$ and $\gamma$ regions are characterized by a hexagonal unit cell with a lattice constant of $6 \AA$ with four protrusions or depressions $3 \AA$ apart. Figure $5.26 \mathrm{c}$ and d display another surface area, where triangular patches denoted $\delta$ again exhibit four protrusion $3 \AA$ apart within the $6 \AA$ unit cell. These triangular patches are separated by the $\varepsilon$ regions that appear darker in the STM images and form no ordered long-range superstructure. Sometimes, further long-range modulations are observed. In general, the long-range periodicities are better developed, if the corresponding structures form larger domains. The surface coverage of these structures depends on the oxidation temperature. For $870 \mathrm{~K}$ oxidation temperature, they cover $10-15 \%$ of the surface, for $930 \mathrm{~K}$ only $5 \%$ and for $1000 \mathrm{~K}$ oxidation temperature they disappear completely and the whole surface is covered by the $\chi$ phase.

\subsubsection{Structure formed at $T=1000 \mathrm{~K}$}

After a final oxidation treatment at $\mathrm{p}\left(\mathrm{O}_{2}\right)=10^{-6}$ mbar and $\mathrm{T}=1000 \mathrm{~K}$ for 15 minutes, the structure $\chi$ is formed on the entire surface, independent of the film growth temperature. The formation of one single $\mathrm{Fe}_{3} \mathrm{O}_{4}(111)$ surface termination agrees also with observation of only one step height of $4.8 \pm 0.2 \AA$ or multiples thereof. This corresponds to the double spacing of $O$ layers along the [111] direction in $\mathrm{Fe}_{3} \mathrm{O}_{4}$, i.e., the distance between equivalent surface terminations. Based on a dynamical LEED intensity analysis, Barbieri et al. $[179,185]$ have proposed a surface structure model for $\mathrm{Fe}_{3} \mathrm{O}_{4}(111)$ films prepared in a similar way. However, the large R-factor of 0.46 left some doubt about the reliability of this structure determination. Therefore, the surface analysis was repeated for five different $\mathrm{Fe}_{3} \mathrm{O}_{4}(111)$ films both by atomic-resolution STM measurements and by dynamical LEED intensity calculations. 


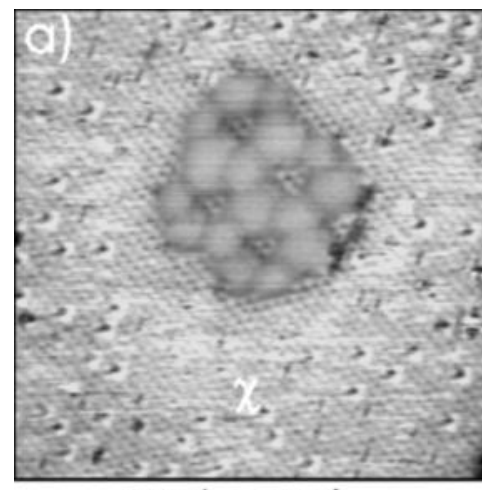

$240 \AA \times 240 \AA$

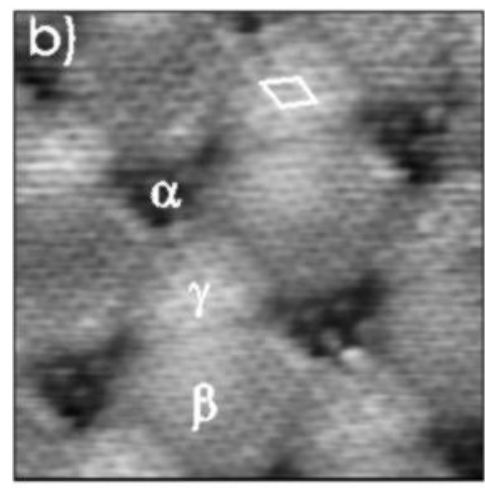

$86 \AA \times 86 \AA$

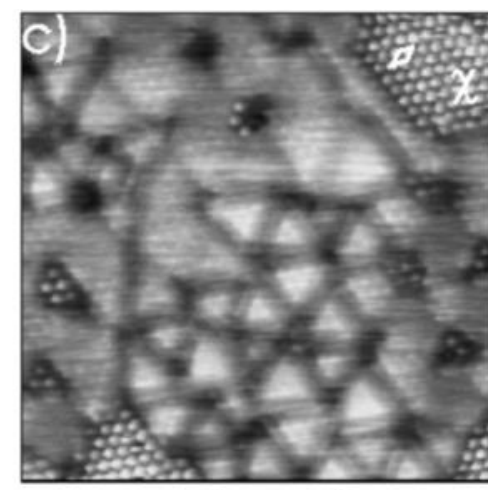

$200 A \times 200 A$

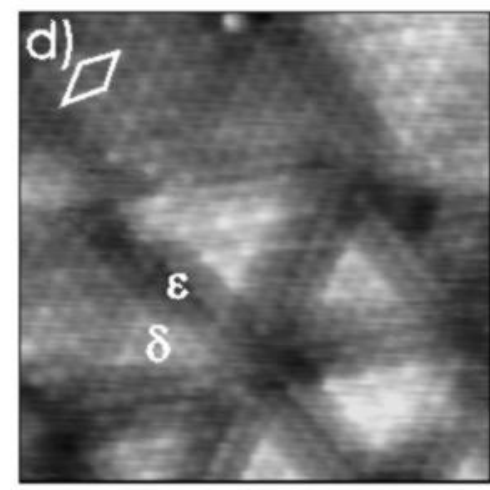

$70 \AA \times 70 \AA$

Fig. 5.26. Atomic resolution STM images of $\mathrm{Fe}_{3} \mathrm{O}_{4}(111)$ film oxidized at $920 \mathrm{~K}$ in $10^{-6} \mathrm{mbar} \mathrm{O}_{2}$. (b) and (d) show magnified areas from (a) and (c), respectively. Hexagonal unit cells with lattice constant of $6 \AA$ are indicated. $\alpha, \beta, \gamma$, and $\delta$ denote regions with different surface structures exhibiting four protrusions $3 \AA$ apart within $6 \AA$ unit cell. In (c) such regions are separated by $\varepsilon$ regions. Different regions arrange in patches forming periodic superstructures or non-periodic long-range modulations. About $90 \%$ of entire surface is covered by region $\chi$, which exhibits no long-range modulation and one protrusion within each $6 \AA$ unit cell. All images were taken at identical tunneling parameters $\mathrm{U}_{\mathrm{t}}=0.3 \mathrm{~V}$, $\mathrm{I}_{\mathrm{t}}=0.3 \mathrm{nA}$. After [277].

\section{(i) Notation for the spinel structure}

The cubic inverse spinel structure of $\mathrm{Fe}_{3} \mathrm{O}_{4}$ is displayed in Fig. 5.27 in a perspective side-view exposing a (111) surface plane. Close-packed $\mathrm{O}$ layers form a cubic $\cdots$ ABCABC $\cdots$ stacking sequence along the [111] direction, indicated by the capital letters A, B, and C. Each letter denotes the lateral registry of the two-dimensional $(1 \times 1)$ unit cell, which has a lattice constant of $5.94 \AA$ and contains four $\mathrm{O}$ atoms (see top view in Fig. 5.2). As pointed out in Section 5.1, three of them are symmetrically equivalent, $\mathrm{O}_{\mathrm{a}}$, and one differs, $\mathrm{O}_{\mathrm{b}}$. Since their vertical coordinates differ by ony $0.04 \AA$, they are treated here as one $\mathrm{O}$ layer. The Fe layers are denoted by lower case letters $\mathrm{a}, \mathrm{b}$, and $\mathrm{c}$ to indicate their lateral registries. The subscipts give the number of atoms within the $(1 \times 1)$ unit cell. For four atoms per unit cell no subscript is given. The first letter denotes the topmost surface layer, so that the termination shown in Fig. 5.27 is described by $a_{1} \mathrm{Ba}_{3} \mathrm{Ca}_{1} \mathrm{~b}_{1} \mathrm{c}_{1} \mathrm{Ac}_{3} \mathrm{~B} \cdots$.

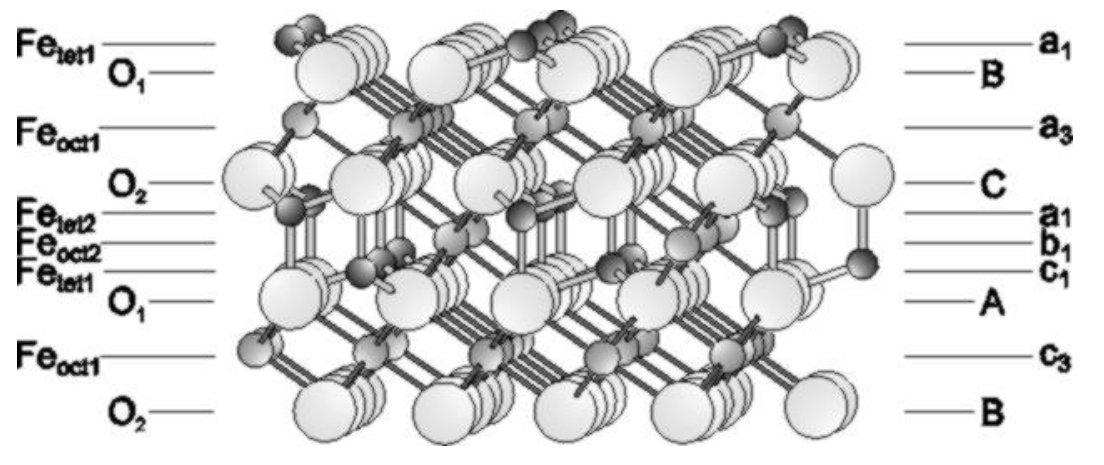

Fig. 5.27. Perspective side view of $\mathrm{Fe}_{3} \mathrm{O}_{4}$ structure exposing (111) surface plane. Two types of notations for layer stacking along [111] direction are given on left and right sides. 
The six bulk terminations indicated on the left side of Fig. 5.27 can be obtained by cutting the spinel (111) stacking sequence. They expose tetrahedrally coordinated $\mathrm{Fe}$ atoms $\mathrm{Fe}_{\text {tet } 1}$ or $\mathrm{Fe}_{\text {tet } 2}$, octahedrally

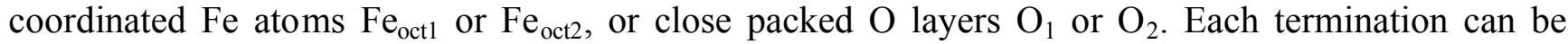
described by the stacking sequence notation indicated on the right side. For example, an $\mathrm{Fe}_{3} \mathrm{O}_{4}(111)$ surface terminated by an $\mathrm{Fe}_{\text {oct } 1}$ layer is denoted by $\mathrm{a}_{3} \mathrm{Ca}_{1} \mathrm{~b}_{1} \mathrm{c}_{1} \mathrm{Ac}_{3} \mathrm{~B} \cdots$. The stacking sequence of planes along the [111] direction can also be described by $\mathrm{Fe}_{\text {tet1 } 1}-\mathrm{O}_{1}-\mathrm{Fe}_{\text {oct1 }}-\mathrm{O}_{2}-\mathrm{Fe}_{\text {tet2 }}-\mathrm{Fe}_{\text {oct2 }} \cdots$.

\section{(ii) LEED calculations}

In dynamical LEED calculations, the diffraction beam intensities $I$ are calculated as a function of the electron energy in $\mathrm{eV}$ and compared to the experimental intensity-voltage $(I-V)$ curves. The scattering properties of the solid are modeled by a muffin-tin potential, usually derived from selfconsistent atomic orbitals [257]. The potential variation normal to the surface is represented by a sharp step, which is modeled by the real part of the inner potential $\mathrm{V}_{0}$. The inelastic scattering and the corresponding mean free path of the incoming electrons is modeled by the imaginary part of the inner potential $\mathrm{V}_{0 \mathrm{i}}$. Thermal vibrations of the atoms are described by a Debye-Waller factor with a fixed Debye temperature, which also modifies the scattering phase shifts.

The LEED calculations were performed using the Barbieri/Van Hove Symmetrized Automated Tensor LEED (SATLEED) package [279]. The theory of this perturbation method was mainly developed by Rous and Pendry [280], and a detailed computational description is given in [281, 282]. In a first step, a full dynamical calculation is performed for a chosen reference structure, which gives the quantities needed to construct a tensor. With this tensor, $I-V$ curves for a variety of models in the proximity of the reference structure can be calculated approximately with little cost of computer time. The search for the best structure model is performed by an automated algorithm, where the agreement between theoretical and experimental I-V curves was quantified by the Pendry R-factor [283]. Since the tensor calculations are perturbative, care has to be taken if atomic coordinates in the optimized model deviate by more than about $0.1 \AA$ from the starting reference structure. In this case, a new reference structure with the geometry of the optimized model has to be defined, a new full dynamical calculation has to be performed, followed by the tensor search in the proximity of the new reference structure. This procedure is repeated until the final structure obtained by the tensor search deviates by less than a few hundredths of an Ångström from the last reference structure. In order to rule out alternative models with larger R-factors, the variance at the R-factor minimum of the best-fit structure $\Delta R$ was used as a confidence interval, where $\Delta R$ is calculated under the assumption of random, noncorrelated errors [283]. Since large relaxations have to be expected for a polar metal-oxide surface, such as $\mathrm{Fe}_{3} \mathrm{O}_{4}(111)$, a large number of reference structure calculations with a subsequent search for an $\mathrm{R}$-factor minimum in the vicinity of each reference structure has to be performed in order to reliably test a single surface termination. All reasonable surface terminations have to be tested, in order to find a model that confidentially corresponds to the true surface structure. For this, a shell script was written, which automatically creates reference structures with geometries varied in a systematic way, for which the full dynamical calculations with the subsequent tensor searches were performed.

The scattering phase shifts were calculated for $\mathrm{Fe}^{2+}$ and $\mathrm{O}^{2-}$ ions in the $\mathrm{FeO}$ sodium chloride structure as described in [185]. Some (111) layers in the $\mathrm{Fe}_{3} \mathrm{O}_{4}$ structure are separated by less than $1 \AA$ (for example $0.6 \AA$ spacing between $\mathrm{a}_{1}$ and $\mathrm{b}_{1}$ layers), which is too close to assure convergence of the multiple scattering computations between these layers. Therefore, composite layers conisting of several atomic planes with larger spacings inbetween [185] were defined. For example, the $\mathrm{a}_{1} \mathrm{Ba}_{3} \mathrm{Ca}_{1} \mathrm{~b}_{1} \mathrm{c}_{1} \mathrm{Ac}_{3} \cdots$ termination in Fig. 5.27 was separated into the $\mathrm{a}_{1} \mathrm{Ba}_{3}$ slab as the surface composite layer, while the $\mathrm{Ca}_{1} \mathrm{~b}_{1} \mathrm{c}_{1} \mathrm{~A}$ and $\mathrm{c}_{3}$ layers represented the bulk repeat unit. Multiple scattering within each composite layer was treated exactly by the Beeby inversion scheme, the interlayer scattering was described by the renormalized forward scattering approximation [278]. The search for the best 
structure was performed with phase shifts for $1 \leq 6$, a temperature of $2 \mathrm{~K}$ and a Debye temperatur of $500 \mathrm{~K}$ for both $\mathrm{O}$ and $\mathrm{Fe}$. It was checked that this number of phase shifts led to no loss in accuracy when compared to more phase shifts. The imaginary part of the inner potential $\mathrm{V}_{0 \mathrm{i}}$ was fixed to a value of $5 \mathrm{eV}$, the real part $\mathrm{V}_{0}$ was fitted within each calculation starting from a value of $10 \mathrm{eV}$. In the final refinement of the best structure, the temperature of calculation was set to the experimental sample temperature of $120 \mathrm{~K}$, the number of phase shifts was increased from $1_{\max }=6$ to 7 , and the imaginary part of the inner potential, as well as the Debye temperatures for both Fe and $\mathrm{O}$ atoms, were optimized.

\section{(iii) Experimental I-V curves}

In order to check the reliability of the experimental data, $I-V$ curves of 5 different $\mathrm{Fe}_{3} \mathrm{O}_{4}(111)$ films were measured, which were grown at oxidation temperatures of $870 \mathrm{~K}$ or $920 \mathrm{~K}$ and finally annealed to $\mathrm{T}=1000 \mathrm{~K}$ for $15 \mathrm{~min}$ in $10^{-6} \mathrm{mbar}_{2}$. One film displayed a LEED pattern with sixfold symmetry although a threefold symmetry is expected for an $\mathrm{Fe}_{3} \mathrm{O}_{4}(111)$ surface. The LEED patterns of the other films deviated from sixfold symmetry in different ways. This is due to coexisting $\mathrm{Fe}_{3} \mathrm{O}_{4}(111)$ domains rotated by $180^{\circ}$ as observed with STM during the $\mathrm{Fe}_{3} \mathrm{O}_{4}$ island growth described in Section 5.4. In each film, these domains cover different surface areas leading to different $I-V$ curves. This problem was solved by averaging the symmetrically inequivalent beams of each diffraction order, for example the (10) and (01) beams, which considerably reduced the total size of the database. After this averaging, the data of all films were very similar. In combination with STM measurements the remaining differences could be assigned to the concentration of atomic point defects at the surface, as discussed below.

\section{(iv) Selection of the best model}

Since $(2 \times 2)$ reconstructed $\mathrm{FeO}(111)$ surface structures could be ruled out convincingly by Barbieri et al. [185], we restricted our search to models for the $\mathrm{Fe}_{3} \mathrm{O}_{4}(111)$ surface with $\mathrm{p} 3 \mathrm{~m} 1$ symmetry. A large number of models was tried [284]. Those which yielded a Pendry R-factor $\mathrm{R}_{\mathrm{P}}<0.5$ in a first optimization cycle are listed in Table 5.3, together with the number of varied structural parameters. The two columns on the right give the surface excess charge $\mathrm{q}_{\mathrm{s}}$ and the effective number of dangling bonds, which will be discussed below. The size of the database was $1000 \mathrm{eV}$ in this first search, and the vertical coordinates of the top-most 12 to 15 atoms per surface unit cell were varied.

TABLE 5.3. Surface terminations considered in first search of dynamical LEED analysis. Those yielding Pendry $R$-factors $R_{P}<0.5$ in a first search are printed bolt face. Notation is explained in text. Also listed are number of structural parameters, surface excess charges $q_{S}$, and effective number of dangling bonds for each termination. From [284].

These models were refined in a second step of the search. Many different reference structures were defined, with the spacing between the first two or three layers was varied in steps of $0.15 \AA$ over total ranges of at least $1 \AA$. Again the vertical coordinates of the topmost 12 to 15 atoms per surface unit cell were varied. This search was performed independently for the reduced data set used in the first step and for two other data sets over $1300 \mathrm{eV}$. All three data sets led to the same best-fit structure, which was model \#1 with strong interlayer relaxations. This structure also was obtained after starting the search with different reference structures. The next best models were \#2 and \#9, which can clearly be ruled out, based on the variance $\Delta R$ at the $R$-factor minimum.

For the final refinement the $1300 \mathrm{eV}$ data set of the film, which had the lowest concentration of point defects, as known from STM measurements on the films. The calculation was performed for the experimental sample temperature of $120 \mathrm{~K}$. The imaginary part of the inner potential was optimized to 
$4 \mathrm{eV}$, the Debye temperatures for Fe and $\mathrm{O}$ were optimized independently to $280 \mathrm{~K}$ and $520 \mathrm{~K}$, respectively. Figure 5.28 shows the comparison between the experimental and calculated $I-V$ curves for the final best-fit structure, which revealed an $R$-factor $R_{p}=0.20$.

\begin{tabular}{|c|c|c|c|c|c|}
\hline \multicolumn{2}{|r|}{ Model } & \multirow{2}{*}{$\begin{array}{c}\# \\
\text { parameters } \\
9 \\
\end{array}$} & \multirow{2}{*}{$\begin{array}{r}R_{P} \\
0.42 \\
\end{array}$} & \multirow{2}{*}{$\begin{array}{r}\mathrm{q}_{\mathrm{S}}[\mathrm{e}] \\
-1.25 \\
\end{array}$} & \multirow{2}{*}{$\begin{array}{c}\begin{array}{c}\text { eff } \# \text { of } \\
\text { dangling bonds }\end{array} \\
6.6 \\
\end{array}$} \\
\hline 1 & $\mathbf{a}_{1} \mathbf{B a}_{3} \mathrm{Ca}_{1} \mathbf{b}_{1} \mathbf{c}_{1} \mathbf{A} \ldots$ & & & & \\
\hline 2 & $a_{1} B_{3} a_{3} C a_{1} b_{1} c_{1} A \cdots$ & 8 & 0.48 & +0.75 & 7.8 \\
\hline 3 & $\mathrm{c}_{1} \mathrm{Ba}_{3} \mathrm{Ca}_{1} \mathrm{~b}_{1} \mathrm{c}_{1} \mathrm{~A} \cdots$ & 9 & 0.55 & -1.75 & 7.2 \\
\hline 4 & $\mathrm{c}_{1}(0.6 \AA) \mathrm{Ba}_{3} \mathrm{Ca}_{1} \mathrm{~b}_{1} \mathrm{c}_{1} \mathrm{~A} \cdots$ & 9 & 0.65 & -1.75 & 7.2 \\
\hline 5 & $\mathrm{c}_{1} \mathrm{~B}_{3} \mathrm{a}_{3} \mathrm{Ca}_{1} \mathrm{~b}_{1} \mathrm{c}_{1} \mathrm{~A} \cdots$ & 8 & 0.63 & +0.25 & 8.4 \\
\hline 6 & $\mathrm{c}_{1}(0.6 \AA) \mathrm{B}_{3} \mathrm{a}_{3} \mathrm{Ca}_{1} \mathrm{~b}_{1} \mathrm{c}_{1} \mathrm{~A} \cdots$ & 8 & 0.50 & +0.25 & 8.4 \\
\hline 7 & $\mathrm{c}_{1} \mathrm{a}_{1} \mathrm{Ba}_{3} \mathrm{Ca}_{1} \mathrm{~b}_{1} \mathrm{c}_{1} \mathrm{~A} \cdots$ & 7 & 0.56 & +1.25 & 6.6 \\
\hline 8 & $\mathrm{c}_{1} \mathrm{a}_{1} \mathrm{~B}_{3} \mathrm{a}_{3} \mathrm{Ca}_{1} \mathrm{~b}_{1} \mathrm{c}_{1} \mathrm{~A} \cdots$ & 9 & 0.60 & +3.25 & 7.8 \\
\hline 9 & $\mathrm{Ba}_{3} \mathrm{Ca}_{1} \mathrm{~b}_{1} \mathbf{c}_{1} \mathrm{~A} \cdots$ & 8 & 0.38 & -4.25 & 10.2 \\
\hline 10 & $\mathbf{B}_{3} a_{3} C_{a_{1}} b_{1} c_{1} A \cdots$ & 7 & 0.38 & -2.25 & 11.4 \\
\hline 11 & $\mathrm{~B}_{1} \mathbf{a}_{3} \mathrm{Ca}_{1} \mathbf{b}_{1} \mathbf{c}_{1} \mathbf{A} \cdots$ & 9 & 0.37 & +1.75 & 7.8 \\
\hline 12 & $\mathrm{C}_{1}(1.2 \AA) \mathrm{a}_{3} \mathrm{Ca}_{1} \mathrm{~b}_{1} \mathrm{c}_{1} \mathrm{~A} \cdots$ & 9 & 0.52 & +1.75 & - \\
\hline 13 & $\mathrm{C}_{1}(1.2 \AA) \mathrm{B}_{1} \mathrm{a}_{3} \mathrm{Ca}_{1} \mathrm{~b}_{1} \mathrm{c}_{1} \mathrm{~A} \cdots$ & 8 & 0.52 & -0.25 & - \\
\hline 14 & $\mathrm{a}_{3} \mathrm{Ca}_{1} \mathrm{~b}_{1} \mathrm{c}_{1} \mathrm{Ac}_{3} \mathrm{~B} \cdots$ & 8 & 0.50 & +3.75 & 9 \\
\hline 15 & $\mathrm{Ca}_{1} \mathrm{~b}_{1} \mathrm{c}_{1} \mathrm{Ac}_{3} \mathrm{~B} \cdots$ & 8 & 0.75 & -3.75 & 9 \\
\hline 16 & $\mathrm{C}_{3} \mathrm{a}_{1} \mathrm{~b}_{1} \mathrm{c}_{1} \mathrm{Ac}_{3} \mathrm{~B} \cdots$ & 7 & 0.68 & -1.75 & 7.8 \\
\hline 17 & $\mathrm{C}_{1} \mathrm{a}_{1} \mathrm{~b}_{1} \mathrm{c}_{1} \mathrm{Ac}_{3} \mathrm{~B} \cdots$ & 9 & 0.73 & +2.25 & 11.4 \\
\hline 18 & $\mathrm{C}_{1} \mathrm{~b}_{1} \mathrm{c}_{1} \mathrm{Ac}_{3} \mathrm{Bc}_{1} \mathrm{a}_{1} \mathrm{~b}_{1} \mathrm{C} \cdots$ & 8 & 0.57 & -0.75 & 7.8 \\
\hline 19 & $\mathrm{~A}_{1} \mathrm{cBa}_{3} \mathrm{Ca}_{1} \mathrm{~b}_{1} \mathrm{c}_{1} \mathrm{~A} \cdots$ & 6 & 0.64 & & \\
\hline 20 & $\mathrm{~A}_{1} \mathrm{c}_{3} \mathrm{Ba}_{3} \mathrm{Ca}_{1} \mathrm{~b}_{1} \mathrm{c}_{1} \mathrm{~A} \cdots$ & 7 & 0.61 & & \\
\hline 21 & $\mathrm{~B}_{1} \mathrm{aCa}_{1} \mathrm{~b}_{1} \mathrm{c}_{1} \mathrm{~A} \cdots$ & 8 & 0.60 & & \\
\hline 22 & $\mathrm{C}_{1} \mathrm{aCa}_{1} \mathrm{~b}_{1} \mathrm{c}_{1} \mathrm{~A} \cdots$ & 8 & 0.51 & & \\
\hline 23 & $\mathrm{C}_{1}(1.2 \AA) \mathrm{B}_{1} \mathrm{aCa}_{1} \mathrm{~b}_{1} \mathrm{c}_{1} \mathrm{~A} \cdots$ & 9 & 0.58 & & \\
\hline
\end{tabular}

The $\mathrm{Fe}_{3} \mathrm{O}_{4}(111)$ surface structure obtained from the LEED analysis is shown in Fig. 5.29. It corresponds to the same termination as obtained by Barbieri et al. [185] with $1 / 4 \mathrm{ML}$ of tetrahedrally coordinated $\mathrm{Fe}_{\text {tet } 1}$ ions exposed in the top layer and a close-packed $\mathrm{O}$ layer underneath. However, the new analysis revealed different surface relaxations, which also are given in Fig. 5.29. The atomic coordinates of this surface structure are given in [284]. 


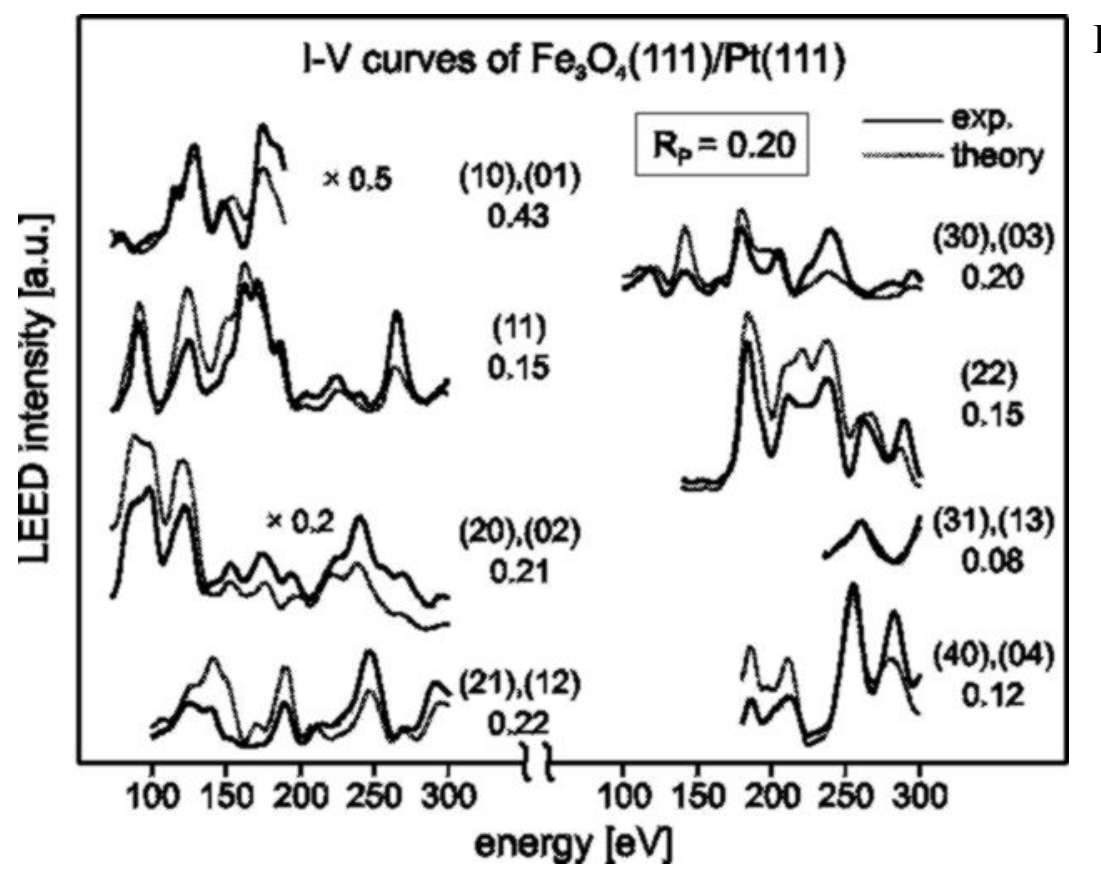

a)

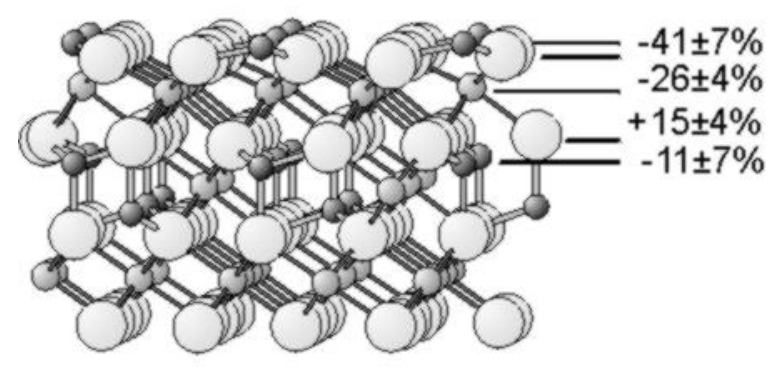

b)

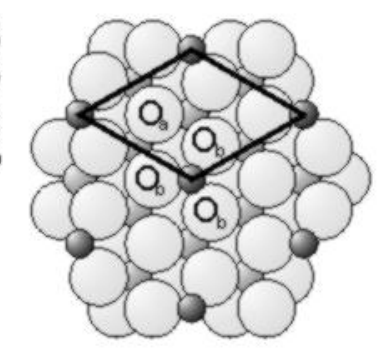

c)

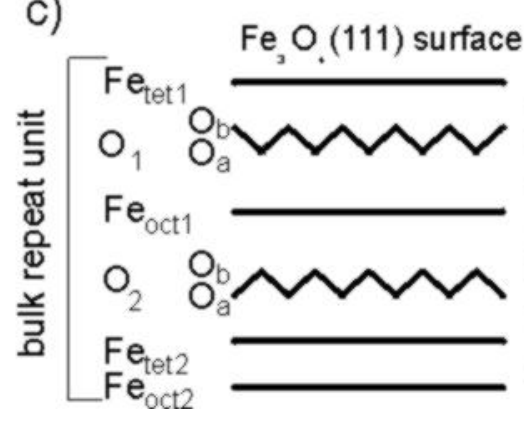

layer distances $[\AA]$

bulk surface

$\begin{array}{ll}0.64 & 0.38 \pm 0.05 \\ 0.04 & 0.08 \pm 0.09\end{array}$

$\begin{array}{ll}1.18 & 0.87 \pm 0.05\end{array}$

$\begin{array}{ll}1.18 & 1.36 \pm 0.05 \quad+15 \pm 4\end{array}$

$0.04 \quad 0.12 \pm 0.09$

$0.64 \quad 0.57 \pm 0.05$

$0.60 \quad 0.60$
Fig. 5.28. Comparison of experimental and theoretical $I-V$ curves for best-fit structure obtained for $\mathrm{Fe}_{3} \mathrm{O}_{4}(111)$ surface, with Pendry $R$-factor of 0.20 for $1300 \mathrm{eV}$ database. From [284].
Fig. 5.29. Side (a) and top (b) views of $\mathrm{Fe}_{3} \mathrm{O}_{4}(111)$ surface structure. Top view is drawn with full ionic sizes, side view with ionic sizes reduced by factor of 0.5. Relative layer relaxations in $\%$ of bulk values are indicated. (c) Schematic side view of relaxed layers with absolute values of bulk

\section{(v) STM measurements}

Figure 5.30 shows atomic-resolution STM images of two $\mathrm{Fe}_{3} \mathrm{O}_{4}(111)$ films grown at oxidation temperatures of $870 \mathrm{~K}$ and $920 \mathrm{~K}$, both finally annealed for 15 minutes at $\mathrm{T}=1000 \mathrm{~K}$ in $10^{-6} \mathrm{mbar}_{2}$. $1 \times 1 \mu \mathrm{m}^{2}$ morphology images of these films are shown in Fig. $5.21 \mathrm{~b}$ and c. On both films, the surface termination $\chi$ (Fig. 5.26) has formed. Atomic steps are $4.8 \AA$ (or multiples thereof) high. Different types of surface point defects are observed. On the film grown at $\mathrm{T}=870 \mathrm{~K}$ (a) their total concentrations is about $50 \%$ and on the film grown at $\mathrm{T}=920 \mathrm{~K}$ (b) it is about $15 \%$. Generally, the 
point-defect concentration decreased with increasing growth temperature. On average, the defect concentration on the films grown at $\mathrm{T}=920 \mathrm{~K}$ was $20 \%$.

According to their appearance in the STM images three types of defects 1-3 in Fig. 5.30a and b can be distinguished. Type 1 defects appear brighter than the hexagonally arranged periodic protrusions, their bright appearence increases in size when going from negative to positive bias voltages. For bias

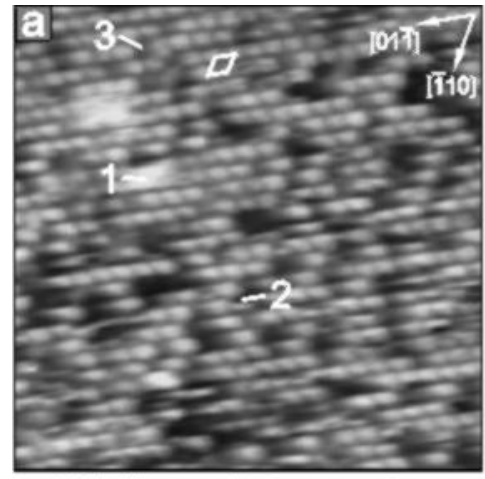

$870 \mathrm{~K}$ growth temperature

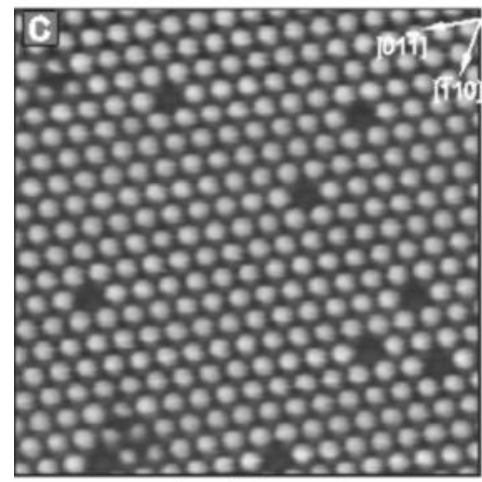

negative bias voltage

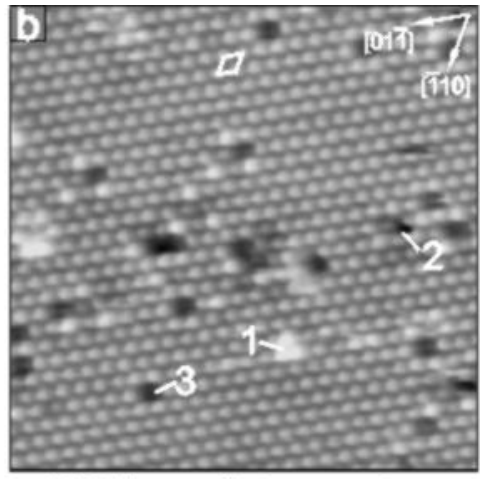

$920 \mathrm{~K}$ growth temperature

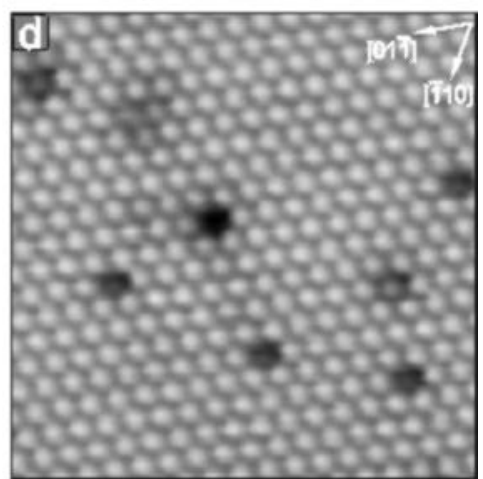

positive bias voltage
Fig. 5.30. (a) and (b) $150 \times 150 \AA^{2}$ images of $\mathrm{Fe}_{3} \mathrm{O}_{4}(111)$ films grown at $870 \mathrm{~K}$ and $920 \mathrm{~K}$, finally annealed for $15 \mathrm{~min}$ at 1000 $\mathrm{K}$ in $10^{-6} \mathrm{mbar}_{2}$. Three types of defects

1-3 are observed. (a) $U_{t}=+0.2 \mathrm{~V}$, $I_{t}=1.7 \mathrm{nA}$. (b) $U_{t}=-0.8 \mathrm{~V}, I_{t}=0.3 \mathrm{nA}$. (c) and (d) $100 \times 100 \AA^{2}$ areas of $\mathrm{Fe}_{3} \mathrm{O}_{4}(111)$ surface grown at $920 \mathrm{~K}$ with only type 3 defects present, taken at different bias voltages. (a) $U_{t}=-0.9 \mathrm{~V}, I_{t}=0.5 \mathrm{nA}$. (b) $U_{t}$ $=+0.5 \mathrm{~V}, I_{t}=0.2 \mathrm{nA}$. From [269].

voltages below $+0.4 \mathrm{~V}$, type 2 defects appear as dark depressions between the periodic protrusions, for bias voltages above $+0.4 \mathrm{~V}$ (not shown) they appear as bright protrusions. Type 3 defects appear as a single missing protrusion of the hexagonal surface lattice at all bias voltages, and represent the dominant type of defect present on all surfaces. The appearance of the regular hexagonal surface lattice as protrusions does also not depend on the bias voltage, which is demonstrated in the images of Fig. 5.30c and d.

Since no contaminants were detected by AES, type 1 and 2 defects could be Fe-oxide clusters, $\mathrm{OH}$ groups or water molecules. LEED calculations [277] indicate the type 3 defects to be missing Fe atoms in the top-most layer. Figure 5.31 compares experimental $I-V$ curves taken from the surfaces with high and low defect density imaged by STM in Fig. 5.30a and b. Although the two data sets look quite similar, an $R$-factor of 0.19 is obtained, when comparing them with each other. The low defect density data revealed the R-factor of 0.20 for the best-fit structure shown in Fig. 5.29, whereas the high defect density data revealed an R-factor of 0.25 for this model. In order to account for the high concentration of type 3 defects, three different types of surface vacancies in concentrations up to $50 \%$ were simulated in LEED intensity calculations. A LEED program that introduces occupation numbers for the surface atoms was used, thereby simulating a random distribution of vacancies on the surface [285]. The removal of $\mathrm{O}_{\mathrm{a}}$ atoms always increased the $R$-factors, the removal of three $\mathrm{O}_{\mathrm{b}}$ atoms together with the Fe atom above revealed only neglegible improvements of the $R$-factors. Removal of the top-most Fe atom considerably improved the $R$-factors, the best-fit resulted in a $12 \%$ reduction of $R_{p}$ for a surface 
defect concentration of $33 \%$. This defect concentration agrees reasonably well with the STM observation in Fig. 5.30a.

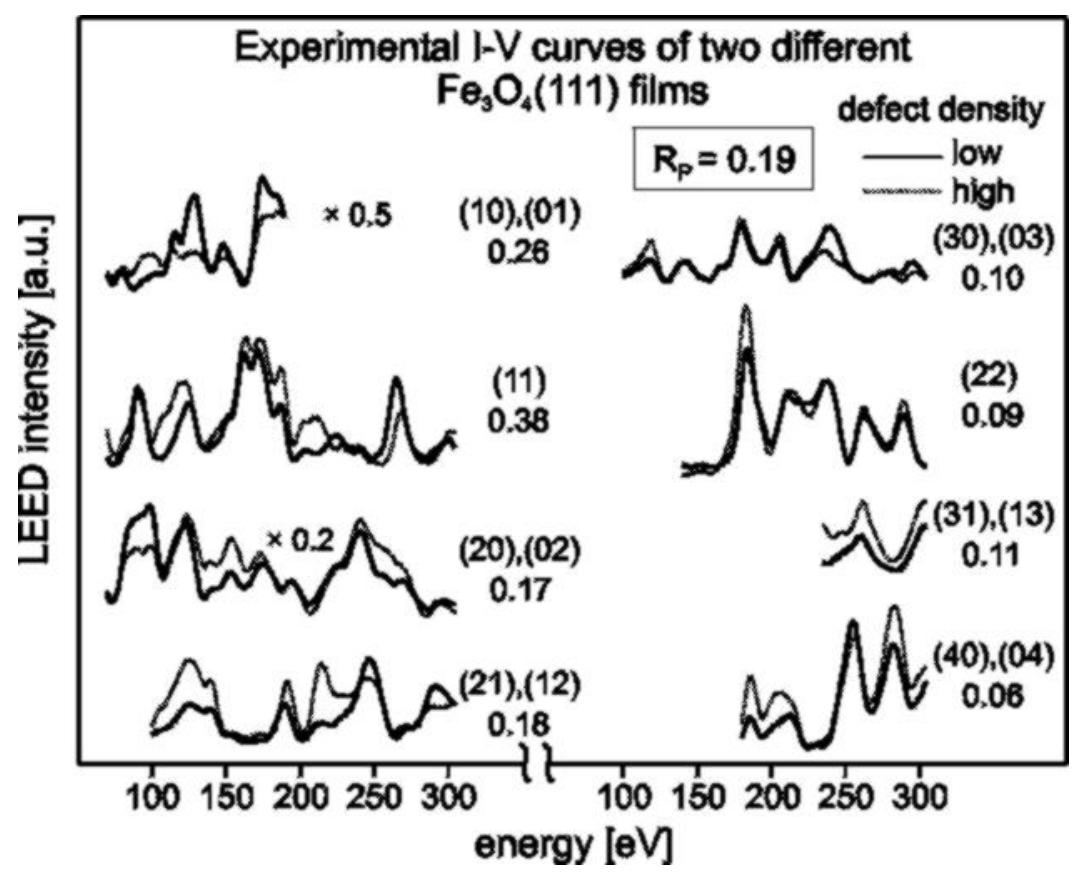

Fig. 5.31. Comparison of experimental $I-V$ curves taken from $\mathrm{Fe}_{3} \mathrm{O}_{4}(111)$ films with high and low defect densities, which were imaged by STM as shown in Fig. $5.30 \mathrm{a}$ and b. After [277].

The LEED analysis, thus, revealed the type 3 defects to be missing Fe atoms in the top-most layer. Since they appear as missing protrusions in the STM images of the $\mathrm{Fe}_{3} \mathrm{O}_{4}(111)$ surface, the hexagonal arranged protrusions observed on the regular surface areas correspond to the positions of $\mathrm{Fe}_{\text {tet } 1}$ atoms, as depicted in Fig. 5.2. This interpretation is also supported by electronic band-structure calculations for $\mathrm{Fe}_{3} \mathrm{O}_{4}$. Upto $1 \mathrm{eV}$ above and below the Fermi level, the $\mathrm{Fe} 3 \mathrm{~d}$ projected density of states is considerably higher, when compared to the $\mathrm{O} 2 \mathrm{p}$ projected density of states $[236,286]$, which also explains the absence of a strong bias-dependency in the STM images of the regular surface.

\subsubsection{Discussion}

\section{(i) Biphase structures formed below $T=1000 \mathrm{~K}$}

For growth temperatures below $1000 \mathrm{~K}, 5-15 \%$ of the surfaces are covered with domains exhibiting different surface structures with four protrusions (or depressions) $3 \AA$ apart within one $6 \AA$ unit cell. These structures arrange in triangular or hexagonal patches that form periodic superstructures or nonperiodic long-range modulations. The formation of these structures seems to depend critically on the preparation conditions. Within the annealing temperature range from 870 to $1000 \mathrm{~K}$ at constant $\mathrm{O}_{2}$ partial pressures of $10^{-6} \mathrm{mbar}$ the portion of these structures decreased with temperature.

Similar structures were observed by Thornton and coworkers, who performed detailed STM studies on several Fe-oxide single-crystal samples. On $\mathrm{Fe}_{3} \mathrm{O}_{4}(111)$ surfaces prepared by $\mathrm{A}^{+}$ion bombardment and annealing to $1100 \mathrm{~K}$ in vacuum, different hexagonal structures with short-range $6 \AA$ and $3 \AA$ periodicities were formed, which are also arranged in triangular and hexagonal patches that form periodic superstructures [287]. This phenomenon was called biphase ordering and interpreted as coexisting domains of $\mathrm{Fe}_{3} \mathrm{O}_{4}(111)$ and $\mathrm{FeO}(111)$ phases, although no $\mathrm{FeO}(111)$ LEED spots could be observed. Detailed models for these structures were proposed, and the formation of FeO was attributed to the reducing preparation conditions applied. The authors also observed regions that looked like the $\chi$ regions in Fig. 5.26, which like they we interpreted as regular $\mathrm{Fe}_{3} \mathrm{O}_{4}(111)$ terminations not reduced to 
FeO. Pure $\mathrm{FeO}(111)$ surfaces could be prepared by continued cycles of sputtering and annealing at $1000 \mathrm{~K}$. On an $\alpha-\mathrm{Fe}_{2} \mathrm{O}_{3}(0001)$ single-crystal, prepared in a similar way, islands forming a superstructure with a periodicity of about $40 \AA$ was formed. This biphase structure was interpreted as coexisting islands of $\alpha-\mathrm{Fe}_{2} \mathrm{O}_{3}(0001)$ and $\mathrm{FeO}(111)$ phases with the latter being formed on top of the underlying $\alpha-\mathrm{Fe}_{2} \mathrm{O}_{3}(0001)$ substrate, because of the reducing preparation conditions applied [138, 288]. The longe-range superstructure was explained by the lattice mismatch between the two types of O sublattices involved.

We have observed that the $\mathrm{Fe}_{3} \mathrm{O}_{4}$-like $\chi$-regions increase in size with increasing annealing temperature. At first glance, this would indicate that higher annealing temperatures represent more oxidizing conditions for a given $\mathrm{O}_{2}$ pressure, which is opposite to what is expected, according to the phase diagram of the Fe- $\mathrm{O}_{2}$ bulk system in Fig. 5.1. However, as discussed in [225], an oxidation of the Fe-terminated $\mathrm{Fe}_{3} \mathrm{O}_{4}(111)$ surface could also result in an $\mathrm{O}$ terminated structure by adsorption of an $\mathrm{O}$ monolayer and a rearrangement of the Fe cations. It would look similar to the $\mathrm{O}$ terminated FeO film surfaces described in Section 5.3 with its $3 \AA$ surface periodicity. The phases with $3 \AA$ surface periodicity would then represent $\mathrm{O}$ terminated $\mathrm{Fe}_{3} \mathrm{O}_{4}$ patches rather than $\mathrm{FeO}$-like phases and, thus, a higher oxidation state. This would also easily explain why no LEED spots corresponding to the $\mathrm{FeO}(111)$ periodicity were observed.

\section{(ii) $\mathrm{Fe}_{3} \mathrm{O}_{4}(111)$ surface structure formed at $\mathrm{T}=1000 \mathrm{~K}$}

After annealing to $1000 \mathrm{~K}$ in $10^{-6}$ mbar $\mathrm{O}_{2}$ partial pressure, one surface termination is formed on epitaxial $\mathrm{Fe}_{3} \mathrm{O}_{4}(111)$ films. The LEED analysis reveals an $R$-factor of $R_{p}=0.20$, which is a very good value, considering the complexity of the $\mathrm{Fe}_{3} \mathrm{O}_{4}$ spinel structure. The surface exposes $1 / 4 \mathrm{ML}$ of $\mathrm{Fe}_{\text {tet } 1}$ atoms exposed in the top-most layer above a close-packed O layer [284]. The same termination was found in a previous LEED analysis [179] but surface relaxations were slightly different. It was checked that it corresponded to a local R-factor minimum in parameter space [284]. The first layer is strongly relaxed inward (41\% when compared to the bulk value), and considerable relaxations up to the fourth interlayer spacing penetrate into the bulk in an oscillating manner (see Fig. 5.29). An inward relaxation of the outermost layer is expected for iono-covalent systems, such as Fe-oxides, when considering both electrostatic and covalent mechanisms that determine the equilibrium interatomic distances for undercoordinated surface atoms in such materials [6]. They were also observed experimentally for the $\mathrm{Cr}_{2} \mathrm{O}_{3}(0001)$ surface [180]. Theoretically, they were predicted for the $\alpha-\mathrm{Fe}_{2} \mathrm{O}_{3}(0001)$ [182] and $\mathrm{Al}_{2} \mathrm{O}_{3}(0001)$ [181] surfaces.

The simple electrostatic and dangling-bond considerations, determining the energetics of metaloxide surfaces, were discussed in Section 2.8. Although all $\mathrm{Fe}_{3} \mathrm{O}_{4}(111)$ terminations represent unstable type 3 surfaces, the (111) plane is a stable surface orientation of magnetite that often occurs on naturally grown crystals [224]. The bulk repeat unit measures $4.85 \AA$ along the [111] direction of $\mathrm{Fe}_{3} \mathrm{O}_{4}$ and extends over six atomic planes (see Fig. 5.27). For the best-fit structure, it is given by the sequence $\mathrm{Fe}_{\text {tet } 1}-\mathrm{O}_{1}-\mathrm{Fe}_{\text {oct1 }}-\mathrm{O}_{2}-\mathrm{Fe}_{\text {tet2 }}-\mathrm{Fe}_{\text {oct2 }}$. Assuming full ionic charges for $\mathrm{Fe}$ and $\mathrm{O}$, it has an electric dipole moment of -6.06 eA perpendicular to the surface, which is caused by an excess surface charge of $-1.25 e$ per $(1 \times 1)$ unit cell. This is one of the smallest excess charges of all $\mathrm{Fe}_{3} \mathrm{O}_{4}(111)$ surface terminations considered in the LEED analysis (cf. Table in [284]). The best-fit structure also has the lowest number of dangling bonds among all models tested. These stability considerations support the termination found in the LEED analysis. Nevertheless, a reduction of the ionic charge density is required on the outer layers to cancel the macroscopic electric field and stabilize the system. Nonstoichiometry on the surface can not account for this, since the dominant type of surface defects are positively charged Fe cation vacancies, which even increase the negative excess surface charge. 
The $\mathrm{Fe}_{3} \mathrm{O}_{4}(111)$ surface must, therefore, be stabilized by the strong interlayer relaxations in the surface region. It is well-known from calculations that the surface density of states is strongly modified on relaxed polar metal-oxide surfaces, when compared to that of the bulk. Often the conduction band of the outermost metal layers is shifted down and the valence-band of the outermost $O$ layers is shifted up in energy, as predicted for the polar $\mathrm{MgO}(111)$ surface [289-291]. For this system the valence and conduction bands even overlap, so that the surface becomes metallic. A large electron transfer from the $\mathrm{O}$ valence to the $\mathrm{Mg}$ conduction band takes place, which reduces the surface charge. For the polar $\mathrm{ZnO}(0001)$ and $(000 \overline{1})$ surfaces no metallization is predicted, but there is a charge transfer that reduces the surface charge and increases the covalency at the surface, which results from shifts of the atomic levels [292]. A smaller bandgap and an increased covalency is also predicted for the $\alpha-\mathrm{Fe}_{2} \mathrm{O}_{3}(0001)$ surface. Also, strong surface states located close to the Fermi level are formed [182]. These surface states also cause a charge redistribution that reduces the excess surface charge and stabilizes the surface. There exist no calculations for the $\mathrm{Fe}_{3} \mathrm{O}_{4}(111)$ surface so far, but most likely the large relaxations found for this surface also change the surface density of states and cause a charge transfer that reduces the excess surface charge, thereby stabilizing this polar termination.

Thornton and coworkers investigated $\mathrm{Fe}_{3} \mathrm{O}_{4}(111)$ and $\alpha-\mathrm{Fe}_{2} \mathrm{O}_{3}(0001)$ single-crystal surfaces that were prepare by $\mathrm{Ar}$ ion bombardment and annealing to $1050 \mathrm{~K}$ in vacuum and in $\mathrm{O}_{2}$ partial pressures between $10^{-7}-10^{-6} \mathrm{mbar}$, with annealing times ranging from 10 to 60 minutes [137, 288, 293]. Both samples exhibited the characteristic $\mathrm{Fe}_{3} \mathrm{O}_{4}(111)$ LEED pattern, and with STM two distinct surface terminations denoted $\mathrm{A}$ and $\mathrm{B}$ were observed. They are separated by steps $3.8 \pm 0.5 \AA$ and $0.5 \pm 0.2 \AA$ high, where both heights are smaller than the distance between equivalent surface terminations of $\mathrm{Fe}_{3} \mathrm{O}_{4}(111)$. Termination A displays atomic resolution STM images that resemble those in Fig. 5.30, where protrusions form a hexagonal lattice with a $6 \AA$ periodicity independent of the bias voltage. Depending on the bias voltage, termination $\mathrm{B}$ also exhibits protrusions with a $6 \AA$ periodicity or honeycomb-like patterns with protrusions $3 \AA$ and $6 \AA$ apart. For termination $\mathrm{B}$, a bulk termination was proposed with $1 / 2 \mathrm{ML}$ of Fe atoms on top $\left(1 / 4 \mathrm{ML} \mathrm{Fe}_{\text {oct } 2}+1 / 4 \mathrm{ML} \mathrm{Fe}_{\text {tet1 }}\right)$, corresponding to the layer sequence $\mathrm{Fe}_{\text {oct2 }}-\mathrm{Fe}_{\text {tet1 }}-\mathrm{O}_{1^{-}} \cdots$ (see Fig. 5.27). Each $\mathrm{Fe}$ atom creates a protrusion in the observed honeycomb pattern. For termination, A a model was proposed where $3 / 4 \mathrm{ML}$ of $\mathrm{Fe}_{\text {oct } 1}$ atoms are located underneath $1 / 4 \mathrm{ML}$ of outermost $\mathrm{O}$ atoms. Each trimer of $\mathrm{Fe}$ atoms is capped by an $\mathrm{O}$ atom creating the protrusion in the STM images with a $6 \AA$ periodicity. According to the notation of Table 5.3, termination $\mathrm{A}$ and $\mathrm{B}$ correspond to models \#11 and \#7, respectively. Based on the LEED analysis presented above they can clearly be ruled out for the surface structure formed on the epitaxial $\mathrm{Fe}_{3} \mathrm{O}_{4}(111)$ films. Whether these different findings are caused by differences between single-crystal samples and epitaxial films, or by the slightly different preparation conditions, has to be clarified in future studies.

\subsection{Surface structures on $\alpha-\mathrm{Fe}_{2} \mathrm{O}_{3}(0001)$ films}

\subsubsection{Pressure dependence of surface structures}

A closed $\mathrm{Fe}_{3} \mathrm{O}_{4}(111)$ film was prepared as described earlier. The sample was then transferred into the high-pressure cell and oxidized for $5 \mathrm{~min}$ at $\mathrm{T}=1000 \mathrm{~K}$ in $10^{-1} \mathrm{mbar} \mathrm{O}_{2}$ for $5 \mathrm{~min}$. In order to quench the structure formed under these conditions, the sample was cooled down to room temperature before pumping off the $\mathrm{O}_{2}$. The sample was transferred back into the STM chamber and analyzed without further annealing. This film exhibited the LEED pattern shown in Fig. 5.3d, which corresponds to an unreconstructed $\alpha-\mathrm{Fe}_{2} \mathrm{O}_{3}(0001)$ surface with a hexagonal unit cell that has a lattice constant of about $5 \AA$. AES, XPS and UPS spectra presented in Section 5.2 confirm the formation of single phased $\alpha-\mathrm{Fe}_{2} \mathrm{O}_{3}$ films. The morphology of these films as depicted in Fig. 5.21d is almost unchanged, when compared to the original $\mathrm{Fe}_{3} \mathrm{O}_{4}(111)$ film. However, the crystallite edges are less 
well-defined. The atomic step density has increased and the atomically flat terraces are not larger than $100 \AA$ in size, which is the consequence of the large mass transport during the phase transformation from $\mathrm{Fe}_{3} \mathrm{O}_{4}$ to $\alpha-\mathrm{Fe}_{2} \mathrm{O}_{3}$. The atomic resolution STM images (Fig. 5.32) exhibit bright spots (protrusions if considered topographically) forming a hexagonal lattice with a $5 \AA$ periodicity. This does not correspond to the periodicity of the close packed $\mathrm{O}$ layer, but to the overall periodicity of the unreconstructed $\alpha-\mathrm{Fe}_{2} \mathrm{O}_{3}(0001)$ surface, which is determined by the Fe layers (cf. Fig. 5.2). The bright spots are less pronounced than, e.g., on the $\mathrm{Fe}_{3} \mathrm{O}_{4}(111)$ surface (Fig. 5.30c) and have varying intensities, which is a characteristic feature of this surface.

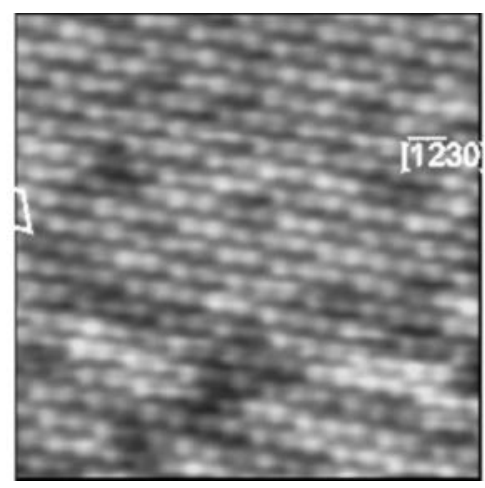

Fig. 5.32. $85 \times 85 \AA^{2}$ STM image of $\alpha-\mathrm{Fe}_{2} \mathrm{O}_{3}(0001)$ surface prepared at $T=1000 \mathrm{~K}$ in $10^{-1} \mathrm{mbar}_{2}$. Indicated hexagonal unit cell has lattice constant of $5 \AA$ and corresponds to unreconstructed $\alpha$ $\mathrm{Fe}_{2} \mathrm{O}_{3}(0001)$ surface structure. $U_{t}=1.5 \mathrm{~V}, I_{t}=1.3$ nA. From [269].

In order to study the influence of $\mathrm{O}_{2}$ partial pressure and temperature on the surface structure, the film was oxidized a second time for $15 \mathrm{~min}$ at $\mathrm{T}=1120 \mathrm{~K}$ in $10^{-3} \mathrm{mbar}_{2}$. The surface order improved, as judged by STM, the step density decreased and the terraces were wider. Figure 5.33a shows a large scale STM image of this film. The terraces are several hundred $\AA$ wide and consist mainly of domains labeled A. Bright islands denoted B with diameters of 20 to $200 \AA$ located predominantly near step edges have formed. The line scans show that these islands apparently are 1 to $1.5 \AA$ higher than the $\mathrm{A}$ domains. Since this is clearly smaller than the distance between identical (0001) surface terminations of $\alpha-\mathrm{Fe}_{2} \mathrm{O}_{3}$, which is $2.29 \AA$, A and $\mathrm{B}$ domains obviously represent different surface terminations. The height difference between equivalent regions $\mathrm{A}-\mathrm{A}$ and $\mathrm{B}-\mathrm{B}$ separated by steps measures $2.3 \AA$, as expected. About $30 \%$ of the entire surface are covered by termination B. Both terminations exhibit bright spots (Fig. 5.33b) forming hexagonal lattices with a periodicity of $5 \AA$. Across A-B domain boundaries, the protrusions are in registry. The termination $\mathrm{A}$ has the same corrugation amplitude as the image of Fig. 5.32. The corrugation profile below the image reveals a step height of about $1 \AA$ between the two terminations and an atomic corrugation amplitude that is larger for termination $\mathrm{A}$ $(\approx 0.3 \AA)$ than termination $\mathrm{B}(\approx 0.1 \AA)$.

Figure 5.33c shows a large scale STM image after oxidizing the film for 15 min in $10^{-4} \mathrm{mbar}_{2}$. Three terraces separated by straight steps are visible. They are not atomically flat, but exhibit irregularly shaped bright and dark patches with lateral dimensions between 20 and $100 \AA$. They could be assigned to $\mathrm{B}$ and $\mathrm{A}$ domains on the basis of their different corrugation and their apparent height difference of about $1 \AA$. Both terminations cover similar areas on the surface. The area covered by termination $\mathrm{B}$ has, thus, increased upon oxidation at a lower $\mathrm{O}_{2}$ pressure. Oxidation in $10^{-5}$ mbar yields a surface consisting almost completely of type B domains.

Figure 5.34 shows a large scale image after an additional oxidation treatment of the sample for $5 \mathrm{~min}$ at $\mathrm{T}=1120 \mathrm{~K}$ in $10^{-6} \mathrm{mbar}_{2}$. A honeycomb superstructure with a periodicity of about $40 \AA$ has formed. The step height is again $2.3 \AA$. This superlattice corresponds to that observed by Condon et al. [138] on $\alpha-\mathrm{Fe}_{2} \mathrm{O}_{3}(0001)$ single-crystal surfaces prepared by repeated cycles of sputtering and annealing at $1100 \mathrm{~K}$ in vacuum followed by a final annealing at $1100 \mathrm{~K}$ in $10^{-6} \mathrm{mbar}_{2}$. It was 
explained by coexisting $\alpha-\mathrm{Fe}_{2} \mathrm{O}_{3}(0001)$ and $\mathrm{FeO}(111)$ domains (biphase structure) arranged in an ordered way, as discussed in Section 5.5C.
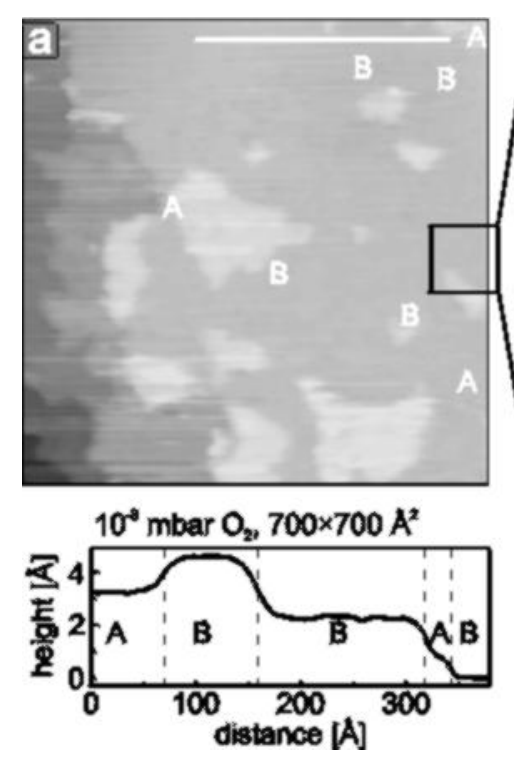
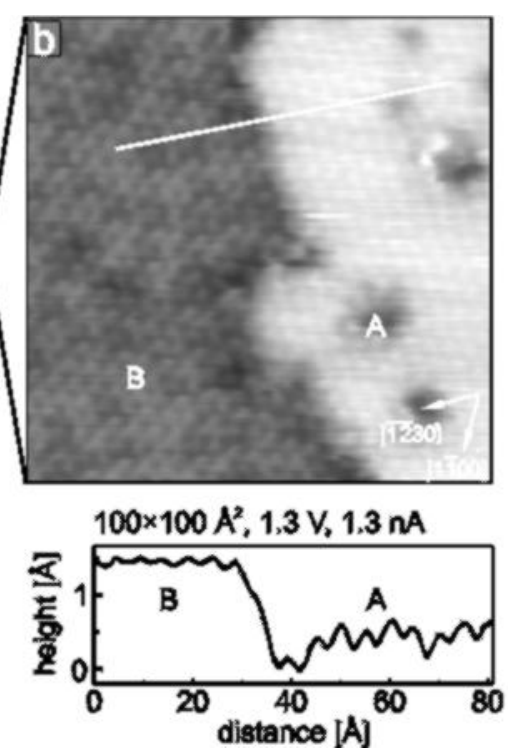

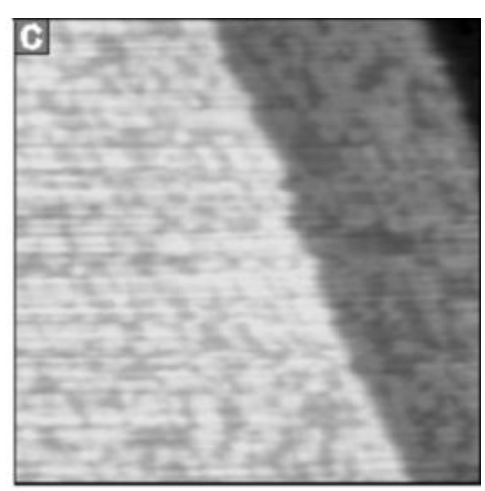

$10^{-4} \mathrm{mbar} \mathrm{O}_{2} 700 \times 700 \AA^{2}$

Fig. 5.33. (a), (b): $\mathrm{STM}$ images of $\alpha-\mathrm{Fe}_{2} \mathrm{O}_{3}(0001)$ film prepared at $\mathrm{T}=1120 \mathrm{~K}$ in $10^{-3}$ mbar $\mathrm{O}_{2}$ partial pressure. Line scans were taken along white lines in images. Differently terminated domains A and B

are separated by apparent height difference of about $1 \AA$. Both exhibit bright spots, which are in registry across domain boundaries and form hexagonal lattices with $5 \AA$ periodicity. (c) After oxidation at $1120 \mathrm{~K}$ in $10^{-4}$ mbar $_{2}$ portion occupied by $\mathrm{B}$ domains has increased but domain size has decreased. From [269].

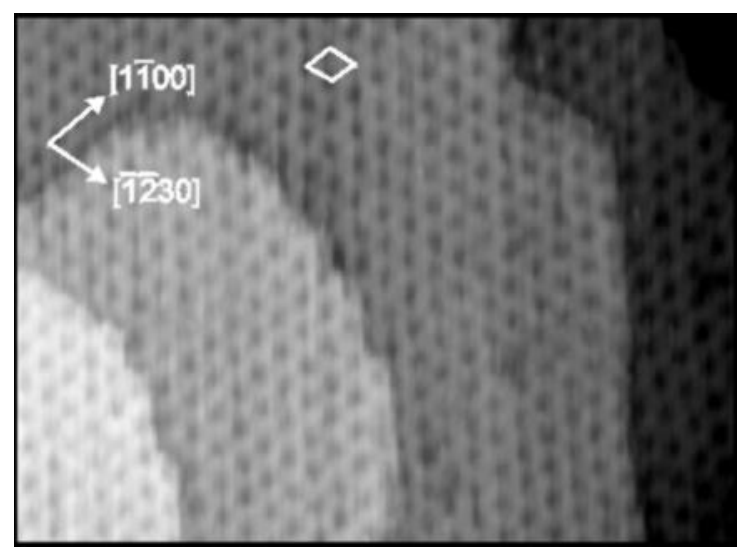

Fig. 5.34. 1000×600 $\AA^{2}$ STM image showing a honeycomb superstructure with periodicity of about $40 \AA$. It is due to biphase structure formed by $\alpha-\mathrm{Fe}_{2} \mathrm{O}_{3}(0001)$ and $\mathrm{FeO}(111)$ domains arranged in ordered manner.

\subsubsection{LEED analysis}

Figure 5.35 shows LEED I-V curves of the $\alpha-\mathrm{Fe}_{2} \mathrm{O}_{3}(0001)$ surfaces prepared by oxidizing in $1 \mathrm{mbar}$ and in $10^{-5}$ mbar $\mathrm{O}_{2}$ representing the almost pure $\mathrm{A}$ and $\mathrm{B}$ termination, respectively. They differ strongly and it is obvious that they represent different structures. The $R_{P}$-factor when comparing them is high (>0.65), which rules out that they are related. Based on calculations presented in the next section it was suggested that structure $A$ was $O$ terminated and structure $B$ was Fe terminated. The adsorption measurements, to be presented in Section 6, suggested an influence of Fe sites on B- 
terminated $\alpha-\mathrm{Fe}_{2} \mathrm{O}_{3}(0001)$, which supported its interpretation as Fe terminated. This was, however, not confirmed by the analysis of the surface structure and composition analysis using LEED and ISS.

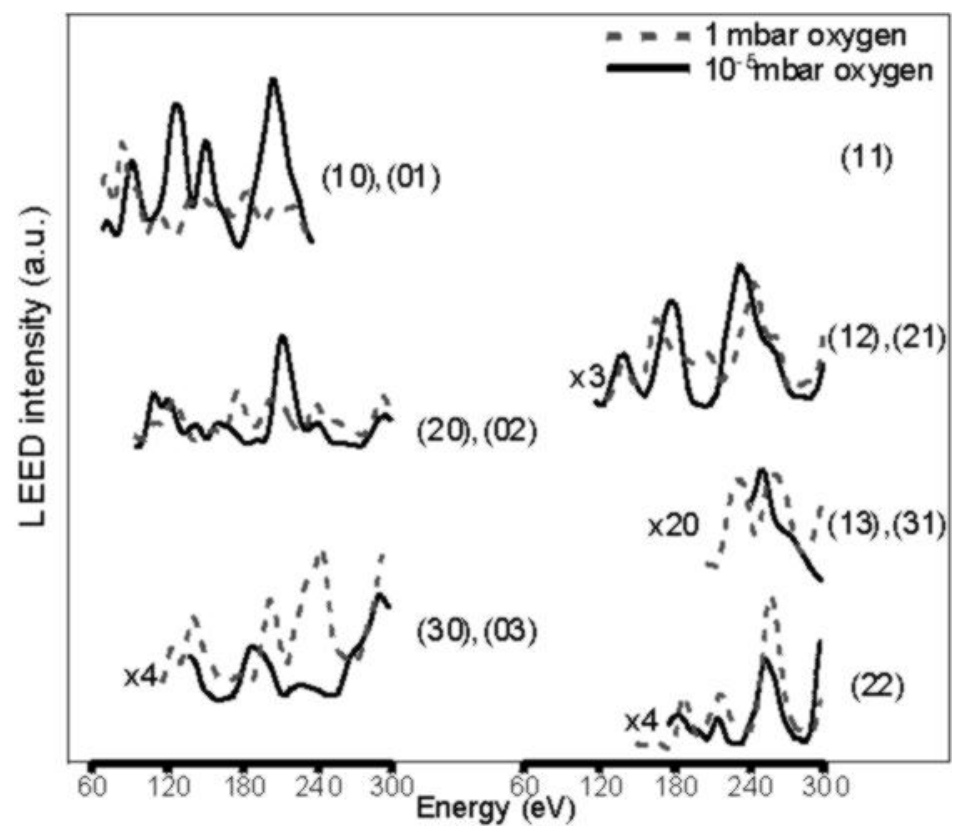

Fig. 5.35. Comparison of LEED $I-V$ spectra for $\alpha-\mathrm{Fe}_{2} \mathrm{O}_{3}(0001)$ prepared in $1 \mathrm{mbar}$ and $10^{-5} \mathrm{mbar} \mathrm{O}_{2}$. From [294].

A fully dynamical LEED analysis, as described in Section 5.5, was performed. As in the case of $\mathrm{Fe}_{3} \mathrm{O}_{4}(111)$, all conceivable initial surface terminations were tested, including structures with changed stacking sequences in the topmost layers. Again, a structural refinement was performed for the structures, which gave the best $R_{P}$-factors in the initial screening search.

\section{(i) Termination A after oxidation in 1 mbar $\mathrm{O}_{2}$}

There are two structures, which fit the LEED data best and have similar values of the $R_{P}$ factor. Both are $\mathrm{O}$ terminated and correspond to the structure in Fig. 5.2c with the top Fe layer removed, but their relaxation is quite different. The best-fit structure is strongly relaxed. The first O-Fe layer distance is reduced by $54 \%$ and the second Fe-Fe layer distance is increased by $46 \%$ (see below, Fig. 5.37). This follows the same rules as for $\mathrm{Fe}_{3} \mathrm{O}_{4}$ and other oxides as discussed in Section 5.52. The $R$ factor is comparatively high $\left(R_{P}=0.38\right)$.

The first interlayer distance in the second best-fit structure is almost unchanged (1\% contraction) but the second one (between the two Fe-layers forming the honeycomb layer, see Fig. 5.2) is reduced by $95 \%$, which means that, within the error bars, these Fe-layers have moved into one plane. The Oatoms of the top layer rearrange in-plane. Always three of them rotate by $3^{\circ}$ around the underlying $\mathrm{Fe}$ atoms. The $R$-factor of this structure is $R_{P}=0.41$. The $R$-factors for the next-best structures are so much higher that they can be ruled out. All structural data and atomic coordinates are given in [294].

The $R$-factor can be reduced considerably $\left(R_{P}=0.29\right)$ by mixing both structures incoherently, so that the real surface structure my consist of a domain mixture of the two structures. One could have expected that these different domains were distinguishable in the STM images, but there is no evidence for this. This may be coincidental for the used parameters (tunneling voltage and current), supported by the fact that the relaxations of all subsurface layers of both structures on the same bulk structure add up in a way that the top O-layers are in the same plane. 


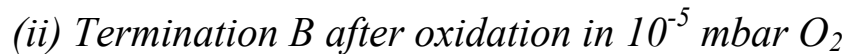

Three surface structures give similarly good $R$-factors. The best-fit structure $\left(R_{P}=0.227\right)$ is also $\mathrm{O}$ terminated. However, the relaxations are very different to that of the A-surface. The first O-Fe layer distance is expanded by $10 \%$, the second Fe-Fe distance is also expanded by $28 \%$ and the third $\mathrm{Fe}-\mathrm{O}$ layer distance is reduced by $24 \%$ (see Fig. 5.37). The next best-fit structure $\left(R_{P}=0.254\right)$ is terminated by a single Fe layer (see Fig. 5.2) and the third structure $\left(R_{P}=0.255\right)$ is terminated by a double Fe layer (honeycomb layer in Fig. 5.2). Also, these structures are strongly relaxed. All structural data and atomic coordinates are given in [294]. Other structure models give clearly higher $R_{P}$-factors and can be ruled out. From the LEED analysis alone, it is not possible to decide, which of these three structures is the true one. If, however, termination A is O terminated, the bright spots in STM correspond to the periodicity of full composite Fe layer beneath. The observation that the bright spots in Fig. 5.33b are in registry when crossing an $\mathrm{A}$ to $\mathrm{B}$ domain boundary would therefore be consistent with a double $\mathrm{Fe}$ or an $\mathrm{O}$ termination of the domains $\mathrm{B}$ but not with a single Fe termination. If, however, an Fe termination would be favourable, a single Fe termination would be plausible because it represents a stable type 2 configuration (see Section 2.8) whereas the double Fe termination would be unstable. The puzzle is resolved by ISS measurements, which confirm an $\mathrm{O}$ termination. Figure 5.36 shows two spectra taken in the $\left[\begin{array}{ll}10 & \overline{1}\end{array}\right]$-azimuth at different incidence angles. Only signals from $\mathrm{O}$ and $\mathrm{Fe}$ are visible. Whereas the Fe signal is fairly strong for an incidence angle nearer to normal, it is reduced to zero for a more grazing incidence, where only scattering from the topmost layer is possible. This proves clearly that $\mathrm{Fe}$ is not contained in the topmost layer.

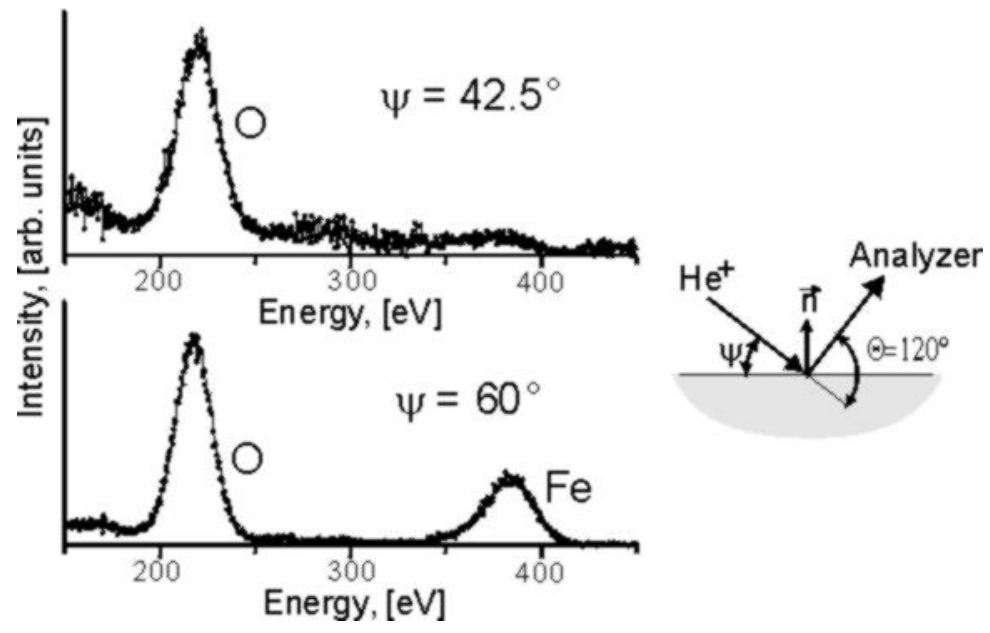

Fig. 5.36. ISS of $\alpha-\mathrm{Fe} 2 \mathrm{O} 3(0001)$ in $[10 \overline{1} 0]$-azimuth for two angles of incidence. For more grazing incidence, $\mathrm{Fe}$ signal disappears completely proving that surface is O terminated [295].

We remain, thus, with an $\mathrm{O}$ terminated surface with a quite strange outward relaxation of the first $\mathrm{O}$ layer. This relaxation would be plausible, if the surface was hydroxylated. Due to its low scattering power, $\mathrm{H}$ in the top layer would not contribute significantly to the LEED diffraction intensity and is therefore almost invisible for LEED. Also, in ISS H cannot be discriminated from the high background in the high-loss region. The outward relaxation found here $(+10 \%)$ lies well within the range found for hydroxilated $\alpha-\mathrm{Al}_{2} \mathrm{O}_{3}(0001)$ [296-299] and $\alpha-\mathrm{Fe}_{2} \mathrm{O}_{3}(0001)$ [300] surfaces ( +3 to $+20 \%$ ).

Figure 5.37 illustrates how hydroxilation may occur by water from the residual gas. Whereas annealing at $p\left(\mathrm{O}_{2}\right)>1$ mbar always results in a closed $\mathrm{O}$ termination, also by oxidizing possibly existing surface $\mathrm{H}$, annealing at lower pressures may lead to an equilibrium concentration of $\mathrm{O}$ vacancies in the top layer. At these vacancies, water from the residual gas may adsorb and dissociate heterolytically to form $\mathrm{OH}^{-}$and $\mathrm{H}^{+}$. The latter would react with a neighbouring $\mathrm{O}$ atom to form a second $\mathrm{OH}^{-}$group. 
Possibly, the bond of adjacent $\mathrm{O}$ atoms to the surface is weakened by such a $\mathrm{OH}$ nucleus, so that further $\mathrm{O}$ is removed and the $\mathrm{OH}$ island grows by further water adsorption as discussed by several authors (see discussion, Section 5.6.4). The lower the $\mathrm{O}_{2}$ pressure, the higher is the concentration of $\mathrm{O}$ vacancies and, thus, the $\mathrm{OH}$ island density. This explains why not only the area occupied by type $\mathrm{B}$ domains increases with decreasing $\mathrm{O}_{2}$ pressure, but also why their density increases (Fig. 5.33c) with the result that their size decreases. At intermediate oxidation pressures, both $\mathrm{A}$ and $\mathrm{B}$ terminations are observed with the A/B occupation ratio increasing continuously with $p\left(\mathrm{O}_{2}\right)$. Since this should not occur for an equilibrium phase transition between two phases (see the discussion in [225]), it must be a result of the reaction kinetics.

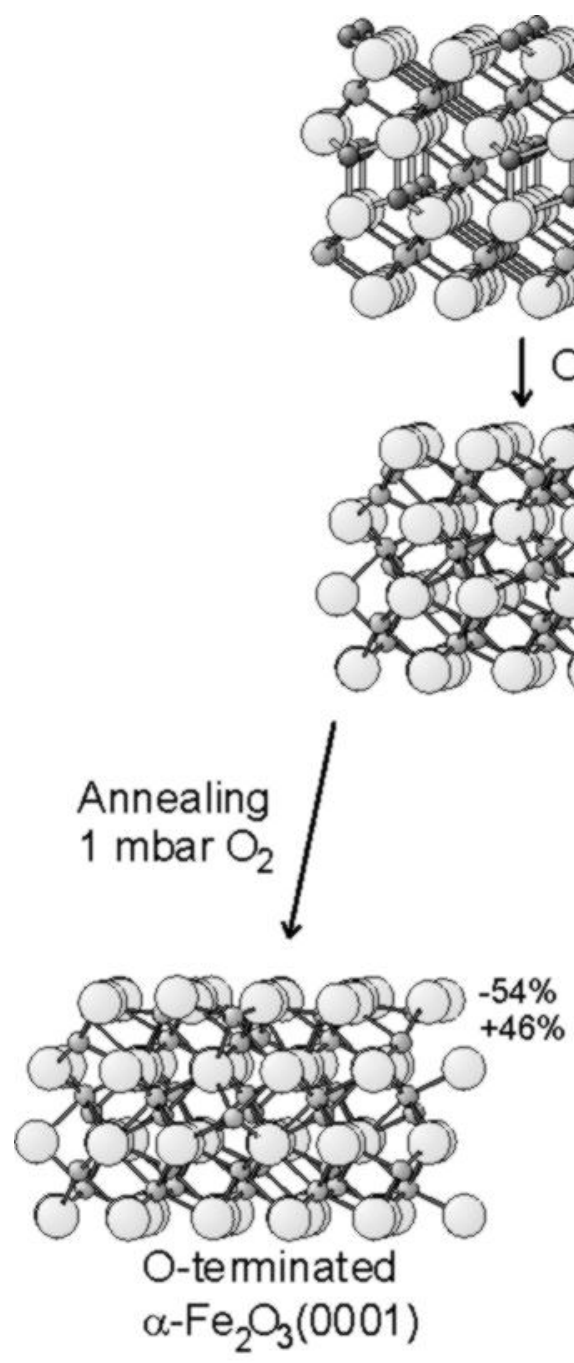

\section{$\mathrm{Fe}_{3} \mathrm{O}_{4}(111)$}

Oxidation $>10^{-3} \mathrm{mbar} \mathrm{O}_{2} / 1100 \mathrm{~K}$

O-terminated $\alpha-\mathrm{Fe}_{2} \mathrm{O}_{3}(0001)$

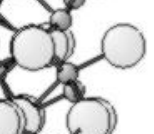

\section{.}
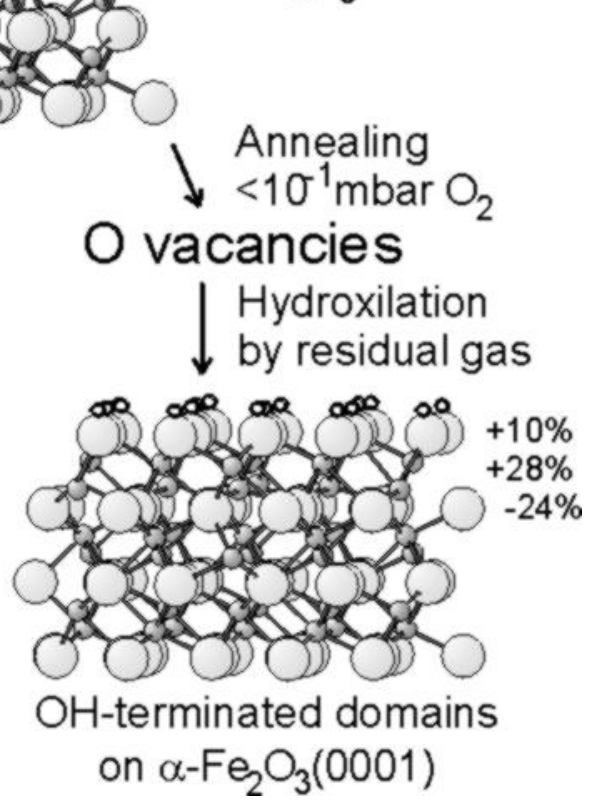

Fig. 5.37. Proposed mechanism for hydroxilation of $\mathrm{O}$ terminated $\alpha$ $\mathrm{Fe}_{2} \mathrm{O}_{3}(0001)$ film after annealing at different $\mathrm{O}_{2}$ pressures. Upon final annealing at low $\mathrm{O}_{2}$ pressure, vacances may be created, which are quickly hydroxilated by water from residual gas [294].

\section{(iii) Mixed structures}

The $I$ - $V$-curves of surfaces formed at intermediate $\mathrm{O}_{2}$ pressures, consisting of mixed $\mathrm{A}$ and $\mathrm{B}$ domains during oxidation (Fig. 5.33), can well be reproduced as a weighted sum of the $I$ - $V$-curves of the pure high- and low-pressure structures. It appears, however, that the A domains in the mixed structures consist of the best-fit structure only and do not contain contributions of the next best-fit structure. This would imply that the next best-fit structure is hydroxilated preferentially. Although experimental evidence still is lacking, we anticipate that the hydroxilated domains are chemically less active so that at least the chemisorption properties of mixed structures would be dominated by the best- 
fit structure of the A domains. This is supported by the comparison of adsorption energies in Section 6.4 .

\subsubsection{Calculations}

As shown in Fig. 5.2, the $\alpha-\mathrm{Fe}_{2} \mathrm{O}_{3}$ structure can be viewed as a stack of hexagonal $\mathrm{Fe}$ and $\mathrm{O}_{3}$ layers along the [0001] axis, $\cdots-\mathrm{Fe}-\mathrm{Fe}-\mathrm{O}_{3}-\mathrm{Fe}-\mathrm{Fe}-\mathrm{O}_{3} \cdots$. While the Fe layers form a unit cell with a lattice constant of $5.0 \AA$, the $\mathrm{O}$ layers form a unit cell with a lattice constant of $2.9 \AA$. The atomic density in the $\mathrm{O}$ layers is three times that of the Fe layers, which is indicated by the subscript 3.

$\mathrm{Ab}$-initio spin-density functional theory calculations were performed for the $\alpha-\mathrm{Fe}_{2} \mathrm{O}_{3}(0001)$ surface [182]. The Gibbs free energy $\Omega$ of a slab at temperature T and $\mathrm{O}_{2}$ partial pressure $p$ is given by

$$
\Omega=E^{\text {total }}+p V-T S-\mu_{\mathrm{Fe}} N_{\mathrm{Fe}}-\mu_{\mathrm{O}} N_{\mathrm{O}} .
$$

$E^{\text {total }}$ is the total energy of the slab, $\mu_{\mathrm{Fe}}$ and $\mu_{\mathrm{O}}$ are the chemical potentials of $\mathrm{Fe}$ and $\mathrm{O}$, respectively. $N_{\mathrm{Fe}}$ and $N_{\mathrm{O}}$ are the numbers of $\mathrm{Fe}$ and $\mathrm{O}$ atoms in the supercell. For typical pressures and temperatures the $p V$ and $T S$ terms can be neglected. The $\mathrm{Fe}$ and $\mathrm{O}$ chemical potentials are related to each other by the existence of the $\mathrm{Fe}_{2} \mathrm{O}_{3}$ bulk phase, which gives

$$
\Omega=E^{\text {total }}-1 / 2 N_{\mathrm{Fe}} \mu_{\mathrm{Fe} 2 \mathrm{O} 3 \text { (bulk) }}+\left(3 / 2 N_{\mathrm{Fe}}-N_{\mathrm{O}}\right) \mu_{\mathrm{O}},
$$

where $\mu_{\mathrm{Fe} 2 \mathrm{O} \text { (bulk) }}$ is the total energy per bulk $\mathrm{Fe}_{2} \mathrm{O}_{3}$ formula unit. Figure 5.38 shows the results of (5.3) for different surface terminations as a function of $\mu_{\mathrm{O}}-\mu_{\mathrm{O}}$ (gas), which increases with increasing ambient $\mathrm{O}_{2}$ partial pressure. Models with $\mathrm{O}$ vacancies were also considered, such as $\mathrm{O}_{1}-\mathrm{Fe}-\mathrm{Fe} \cdots$, where the subscript denotes an $\mathrm{O}$ terminated surface with only $1 / 3 \mathrm{ML}$ of $\mathrm{O}$ atoms in the topmost layer. $\gamma$ is the Gibbs free energy per surface area. The meaningful range for $\mu_{\mathrm{O}}-\mu_{\mathrm{O}}$ (gas) indicated by the dotted lines is limited by the conditions that the chemical potential of Fe has to be smaller than that of an atom of bulk $\mathrm{Fe}$, and the chemical potential of $\mathrm{O}$ has to be smaller than that of an $\mathrm{O}$ atom in an $\mathrm{O}_{2}$ molecule. Otherwise, an $\mathrm{Fe}$ or $\mathrm{O}_{2}$ condensate will form on the surface.

The calculations reveal a most stable $\mathrm{O}_{3}$-terminated surface under high $\mathrm{O}_{2}$ pressures, and a most stable Fe-terminated surface under low $\mathrm{O}_{2}$ pressures. Large interlayer relaxations were obtained from these calculations, which are listed in Table 5.4. As on $\mathrm{Fe}_{3} \mathrm{O}_{4}(111)$, for the Fe terminated surface Fe$\mathrm{O}_{3}-\mathrm{Fe}-\cdots$ again an inward relaxation of the outermost $\mathrm{Fe}$ layer and alternating relaxations down to the fourth layer are found. For the $\mathrm{O}$ terminated surface $\mathrm{O}_{3}-\mathrm{Fe}-\mathrm{Fe} \cdots$, a huge contraction is found between the two Fe layers underneath the topmost $\mathrm{O}$ layer. These relaxations considerably lower the surface energies, which can be seen from the dashed lines in Fig. 5.38, indicating the energies of the corresponding two unrelaxed terminations. Regarding the electrostatic surface energies in a purely ionic model, the Fe-terminated surface is a stable type 2 surface according to the classification of Tasker. The $\mathrm{O}_{3}$-terminated surface is of type 3 and should be unstable, it must be stabilized by a charge transfer that compensates for the macroscopic electric field. This is most likely caused by the strong surface state obtained from the calculation, which is located below the Fermi level at the Fe layer underneath the outermost $\mathrm{O}$ layer. When occupied, it may reduce the negative excess surface charge, thus, stabilizing the polar $\mathrm{O}_{3}$-terminated surface. Also, for both surfaces increased covalent bonding and smaller bandgaps than in bulk $\mathrm{Fe}_{2} \mathrm{O}_{3}$ are obtained.

In these calculations, the influence of hydrogen or water was not considered. The stability of a hydroxilated in comparison to an $\mathrm{O}$ or Fe terminated surface has therefore not yet been established theoretically for this system. 


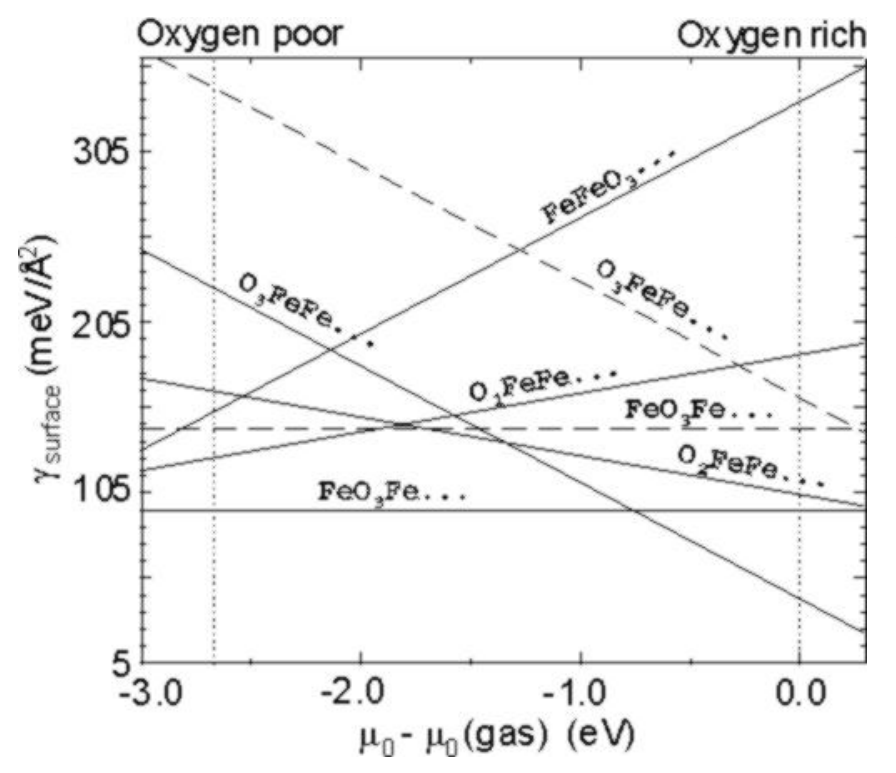

Fig. 5.38. Surface energies of different $\alpha$ $\mathrm{Fe}_{2} \mathrm{O}_{3}(0001)$ surface terminations. $\mu \mathrm{O}$ (gas) is chemical potential per $\mathrm{O}$ atom in molecular $\mathrm{O}_{2}$.

Allowed range of $\mu_{O}-\mu_{O}$ (gas) is indicated by vertical dotted lines, where left one corresponds to strongly Fe-rich (i.e., O-poor) conditions, and right one corresponds to strongly O-rich conditions (i.e., high $\mathrm{O}_{2}$ gas pressure). Full lines show relaxed geometries, and dashed lines give results for unrelaxed surfaces for comparison. From[182].

TABLE 5.4. Interlayer relaxations at $\mathrm{Fe}-\mathrm{O}_{3}-\mathrm{Fe}-\cdots$ and $\mathrm{O}_{3}-\mathrm{Fe}-\mathrm{Fe}-\cdots$ surface terminations of $\alpha$ $\mathrm{Fe}_{2} \mathrm{O}_{3}(0001)$ relative to corresponding bulk spacings obtained from ab-initio calculation [182].

\begin{tabular}{|c|c|c|}
\hline Interlayer & $\mathrm{Fe}_{3}-\ldots$ termination & $\mathrm{O}_{3}-\mathrm{Fe}-\ldots$ termination \\
\hline $\mathrm{Fe}-\mathrm{O}_{3}$ & $-57 \%$ & \\
\hline $\mathrm{O}_{3}-\mathrm{Fe}$ & $+7 \%$ & $-1 \%$ \\
\hline $\mathrm{Fe}-\mathrm{Fe}$ & $-33 \%$ & $-79 \%$ \\
\hline $\mathrm{Fe}-\mathrm{O}_{3}$ & $+15 \%$ & $+37 \%$ \\
\hline $\mathrm{O}_{3}-\mathrm{Fe}$ & $+5 \%$ & $-6 \%$ \\
\hline $\mathrm{Fe}-\mathrm{Fe}$ & $-3 \%$ & $+16 \%$ \\
\hline $\mathrm{Fe}-\mathrm{O}_{3}$ & $-1 \%$ & $-4 \%$ \\
\hline $\mathrm{O}_{3}-\mathrm{Fe}$ & $+4 \%$ & $+4 \%$ \\
\hline
\end{tabular}

\subsubsection{Discussion}

$\mathrm{Ab}$ initio calculations on the $\alpha-\mathrm{Fe}_{2} \mathrm{O}_{3}(0001)$ surface predict different equilibrium surface terminations. At high $\mathrm{O}_{2}$ pressure, a purely $\mathrm{O}$ terminated surface is predicted, whereas at low $\mathrm{O}_{2}$ pressures a termination by a single Fe layer is stabilized. Different surface terminations, depending on the $\mathrm{O}_{2}$ pressure during oxidation at elevated temperature, were also observed experimentally. It seemed straightforward to identify them with the theoretically predicted terminations. The adsorption measurements on the surface prepared at low $\mathrm{O}_{2}$ pressure (presented in Section 6) showed close similarities to those on the Fe terminated $\mathrm{Fe}_{3} \mathrm{O}_{4}(111)$ surface, which also suggested that it was $\mathrm{Fe}$ terminated. A first hint that this interpretation was doubtful came from the observation that the adsorption properties of the differently prepared surfaces did not differ strongly. The dynamical LEED analysis and the ISS measurements presented in Section 5.6.2 gave the final evidence that no $\mathrm{Fe}$ terminated surface had been prepared. Both $\mathrm{A}$ and $\mathrm{B}$ domains were $\mathrm{O}$ terminated. The relaxation on the termination $\mathrm{A}$ (high $\mathrm{O}_{2}$ pressure) agrees roughly with that of the termination predicted theoretically at high $\mathrm{O}_{2}$ pressure. Termination $\mathrm{A}$ is, thus, purely $\mathrm{O}$ terminated $\left(\mathrm{O}_{3}\right)$. The relaxation on termination $\mathrm{B}$, however, is very unusual. The first $\mathrm{O}$ layer relaxes outward, which would destabilize the surface concerning ionicity arguments. Therefore, it is proposed that this surface is hydroxylated. A model is 
proposed, which assumes hydroxilation by water from residual gas, attacking the surface at vacancies in the top $\mathrm{O}$ layer. The equilibrium density of these vacancies should increase with decreasing $\mathrm{O}_{2}$ pressure. A contradiction to the theoretical predictions does not exist, since the influence of $\mathrm{H}$ or water was not included in the calculations.

Although not known exactly, the partial pressure of water was always considerably lower than that of $\mathrm{O}_{2}$ during oxidation. The existence of a hydroxylated surface would therefore imply that hydroxylation is strongly favoured. The Fe terminated $\mathrm{Fe}_{3} \mathrm{O}_{4}(111)$ surface is capable to dissociate water easily (see Section 6.2). Fe terminated $\alpha-\mathrm{Fe}_{2} \mathrm{O}_{3}(0001)$ would expose $1 / 3 \mathrm{ML}$ Fe and it seems plausible that it should be even more reactive for hydroxylation, since no oxidation is necessary to form one of the stable Fe hydroxides.

Indeed, $\alpha-\mathrm{Fe}_{2} \mathrm{O}_{3}(0001)$ hydroxylation was found to be facile [298, 301]. For an Fe terminated and even more for a defective $\mathrm{O}$ terminated $\alpha-\mathrm{Fe}_{2} \mathrm{O}_{3}(0001)$ surface, the hydroxylation energy was calculated to be very large $(-298.1 \mathrm{~kJ} / \mathrm{mol})$ [302]. An Fe terminated surface was found to be completely unstable in presence of excess water $(>67 \%$ coverage) and was predicted to relax, leaving an $\mathrm{O}$ terminated hydrated layer with $\mathrm{Fe}(\mathrm{OH})_{3}$ subunits behind [300]. $\mathrm{OH}$ species on the (0001) face of hematite were found to be difficult to remove (in contrast to surface $\mathrm{OH}$ groups on other hematite planes) and were thermally stable up to at least $1073 \mathrm{~K}$ in $\mathrm{O}_{2}$ atmosphere as evidenced by infrared spectroscopy [303], and $\mathrm{OH}$ on Fe terminated $\alpha-\mathrm{Fe}_{2} \mathrm{O}_{3}(0001)$ could not be removed by excessive heating at $900 \mathrm{~K}$ without reducing the near-surface region to $\mathrm{Fe}(\mathrm{II})$ [301]. Also the isostructural $\alpha$ $\mathrm{Al}_{2} \mathrm{O}_{3}(0001)$ surface has been found to get hydroxilated easily [63, 297, 298, 304-308]. This was confirmed by theory [296].

It is thus very likely that both a defective $\mathrm{O}$ and an Fe terminated surface of $\alpha-\mathrm{Fe}_{2} \mathrm{O}_{3}(0001)$ films are hydroxylated, when the water $/ \mathrm{O}_{2}$ pressure ratio exceeds a certain, possibly low value and that once hydroxylated they are very stable. Whereas an almost defect free purely $\mathrm{O}$ terminated surface may be prepared under sufficiently high $\mathrm{O}_{2}$ pressure, it seems doubtful that the residual gas pressure of water can be suppressed so far that an Fe terminated surface - if once produced - may be stabilized long enough to perform meaningful experiments.

The bright spots observed by STM on both the A and B terminated surfaces do not represent the periodicity of the topmost $\mathrm{O}$ and $\mathrm{OH}$ layers, respectively, but that of the Fe layer(s) beneath. The reason is that the electronic properties of the subsurface Fe are changed significantly by the surface relaxation. Spin-density functional theory [182] showed for the $\mathrm{O}_{3}$-terminated surface that $\mathrm{Fe} 3 \mathrm{~d}$ derived surface states are formed near the Fermi edge, which spatially even extend through the top $\mathrm{O}$ layer. These Fe $3 \mathrm{~d}$ derived states represent the only occupied and unoccupied states near the Fermi edge and are therefore imaged by STM, confirming the suggestion of Eggleston [309]. They may also noticably affect the surface reactivity. The bright spots observed by STM are, thus, no topographic protrusions but are caused completely by electronic effects. The different apparent corrugation amplitudes on $\mathrm{O}$ terminated $\mathrm{A}$ and hydroxylated $\mathrm{B}$ type domains indicate different local densities of states (LDOS). Also, the LDOS of the Fe layers below the top $\mathrm{O}$ or hydroxy layer must be different leading to apparant step heights of 1-1.5 $\AA$ instead of the determined geometric distance between the $\mathrm{O}$ planes of $\sim 0.5 \AA$.

Several previous studies also revealed the $\alpha-\mathrm{Fe}_{2} \mathrm{O}_{3}(0001)$ surface structure to depend critically on the preparation parameters. Henrich and coworkers prepared single-crystals by sputtering and subsequent annealing in $10^{-6} \mathrm{mbar}_{2}$ for 30-60 minutes [135, 310]. Based on the observed LEED pattern they obtained a surface region reduced to $\mathrm{Fe}_{3} \mathrm{O}_{4}$ for annealing temperatures of $1050 \mathrm{~K}$, whereas a LEED pattern, corresponding to a stoichiometric $\alpha-\mathrm{Fe}_{2} \mathrm{O}_{3}(0001)$ surface was obtained after annealing to $1170 \mathrm{~K}$. This must be a surface effect, since it seems to be in contrast to bulk thermodynamics. Thornton and coworkers studied natural $\alpha-\mathrm{Fe}_{2} \mathrm{O}_{3}(0001)$ single-crystal surfaces, which were prepared 
by repeated cycles of sputtering and annealing to $1100 \mathrm{~K}$ in vacuum, followed by a final annealing to $1100 \mathrm{~K}$ in $10^{-6}$ mbar $\mathrm{O}_{2}$. A biphase structure, interpreted as coexisting islands of $\alpha-\mathrm{Fe}_{2} \mathrm{O}_{3}(0001)$ and $\mathrm{FeO}(111)$ phases, was observed. These phases existed in domains forming a superstructure with a periodicity of about $40 \AA[138,288]$. The same superstructure is obtained on the epitaxial film shown in Fig. 5.34 for very similar reducing preparation conditions, indicating the formation of the same structure. Hochella and coworkers studied $\alpha-\mathrm{Fe}_{2} \mathrm{O}_{3}(0001)$ surfaces under polyphenyl ether, which were obtained by fracturing along (0001) in air [134]. Since these surfaces were exposed to air, an $O$ terminated surface or a surface covered by $\mathrm{O}$ and water adsorbates is expected. For negative bias voltages, protrusions forming hexagonal lattices with $3 \AA$ periodicities were observed, positive bias voltages resulted in varying images with $3 \AA$ and $5 \AA$ periodicities. STM image simulations, based on Hartree-Fock calculations, revealed that these protrusions are located at the Fe atom positions of the surface Fe layer in case of an Fe-termination, or at the positions of the second Fe layer in case of an $\mathrm{O}_{3}$-termination. This agrees with the interpretion of the STM protrusions in this work, which is attributed to the $\mathrm{Fe} 3 \mathrm{~d}$ surface states on both the $\mathrm{O}_{3}$-terminated and the hydroxylated $\alpha-\mathrm{Fe}_{2} \mathrm{O}_{3}(0001)$ surfaces.

\subsection{Summary}

Fe-oxides grow in a Stranski-Krastanov mode onto $\mathrm{Pt}(111)$ forming well-ordered films if prepared by repeated cycles of Fe deposition and subsequent oxidation. The initial layer-by-layer growth of $\mathrm{FeO}(111)$ is limited to a maximal thickness of $2.5 \mathrm{ML}$ by the electrostatic surface energy building up with increasing number of Fe-O bilayers. On top of this $\mathrm{FeO}$ film, a homogeneous nucleation of threedimensional $\mathrm{Fe}_{3} \mathrm{O}_{4}(111)$ islands begins. Eventually, these islands coalesce and form $\mathrm{Fe}_{3} \mathrm{O}_{4}(111)$ films, usually at least $100 \AA$ thick, which can be transformed into $\alpha-\mathrm{Fe}_{2} \mathrm{O}_{3}(0001)$ films by a high pressure oxidation treatment. These multilayer films form smooth surfaces on a mesoscopic scale, with vertical roughnesses ranging from $30-150 \AA$ on a lenght scale of $1 \mu \mathrm{m}$. Their morphologies and their atomic step densities can be varied within a certain range by choosing the proper preparation parameters.

While the first $\mathrm{FeO}$ layers represent a two-dimensional system with properties different from $\mathrm{FeO}$ bulk crystals, photoelectron spectroscopy and X-ray absorption measurements indicate that the $\mathrm{Fe}_{3} \mathrm{O}_{4}$ and $\alpha-\mathrm{Fe}_{2} \mathrm{O}_{4}$ multilayer films are equivalent to their corresponding bulk counterparts. This is also confirmed by the dynamical LEED intensity analysis performed for the $\mathrm{Fe}_{3} \mathrm{O}_{4}(111)$ and $\alpha-\mathrm{Fe}_{2} \mathrm{O}_{3}(0001)$ surfaces, which rule out $\mathrm{FeO}$ related models. This agrees with recent Mössbauer spectroscopy and optomagnetic measurements, which also revealed bulk properties for $\mathrm{Fe}_{3} \mathrm{O}_{4}(111)$ multilayer films grown onto $\mathrm{Pt}(111)$ [311].

The three oxides exhibit different LEED patterns from, which they easily can be identified. They all form strongly relaxed, unreconstructed bulk terminated surface structures. The ultrathin $\mathrm{FeO}(111)$ films form four different coincidence structures as the coverage increases. They all are terminated by close-packed $\mathrm{O}$ layers. Compared to bulk $\mathrm{FeO}$, the films are laterally expanded and have reduced interlayer spacings, which reduces their electrostatic surface energies.

The surface structures formed on thicker $\mathrm{Fe}_{3} \mathrm{O}_{4}$ and $\alpha-\mathrm{Fe}_{2} \mathrm{O}_{3}$ films critically depend on the preparation procedure, as observed for many metal-oxide single-crystal samples. However, a welldefined $\mathrm{Fe}_{3} \mathrm{O}_{4}(111)$ surface structure can be prepared, which exposes $1 / 4 \mathrm{ML}$ of $\mathrm{Fe}$ atoms above a hexagonal close-packed $\mathrm{O}$ layer. The outermost Fe layer is strongly relaxed inward as expected for polar surfaces of ionic materials. The dominating type of point defect are missing Fe atoms in the topmost layer.

The structure of $\alpha-\mathrm{Fe}_{2} \mathrm{O}_{3}(0001)$ surfaces depends on the $\mathrm{O}_{2}$ pressures during the oxidation treatment at elevated temperature. In agreement with ab-initio calculations, oxidation at $1 \mathrm{mbar} \mathrm{O}_{2}$ pressure results in an $\mathrm{O}$ terminated surface with strong inward relaxation of the top $\mathrm{O}$ layer. Quite unexpected, 
oxidation at $10^{-5}$ mbar $\mathrm{O}_{2}$ pressure leads to a surface, which apparently is also $\mathrm{O}$ terminated, however, with a quite unusual outward relaxation of the $\mathrm{O}$ layer, which is consistent with hydroxylation. It is likely that this is due to the unavoidable influence of water in the residual gas. The Fe-terminated surface, predicted by ab-initio calculations for low $\mathrm{O}_{2}$ pressure, was therefore not observed so far. The calculation also showed that the electronic structure near the surface is strongly modified for the $\mathrm{O}$ terminated $\alpha-\mathrm{Fe}_{2} \mathrm{O}_{3}(0001)$ surface. Surface states near the Fermi level related to the Fe $3 \mathrm{~d}$ states of the $\mathrm{Fe}$ layer below the top $\mathrm{O}$ layer stabilize this polar surface by reducing the charge in the topmost $\mathrm{O}$ layer. They are also responsible for the apparent corrugation observed in STM, which thus turns out to be caused by electronic and not by topographic properties. These Fe derived surface states also determine the chemical and catalytic surface properties. STM measurements show that different types of atomic surface defects, such as steps, vacancies and modified surface atoms are always present on the Fe-oxide surfaces. These defect sites have different local electronic structures and might be very reactive.

\section{Adsorption studies}

Adsorption studies were performed on 1-2 ML thick $\mathrm{FeO}(111)$ films, on $\mathrm{Fe}_{3} \mathrm{O}_{4}(111)$ multilayer films and on $\alpha-\mathrm{Fe}_{2} \mathrm{O}_{3}(0001)$ films. The $\mathrm{FeO}(111)$ films are $\mathrm{O}$ terminated. The $\mathrm{Fe}_{3} \mathrm{O}_{4}(111)$ were prepared by a final oxidation at $\mathrm{T}=1000 \mathrm{~K}$ in $10^{-6} \mathrm{mbar}_{2}$ resulting in a single termination exposing $1 / 4$ ML of Fe ions above a hexagonal close-packed $\mathrm{O}$ layer. The $\alpha-\mathrm{Fe}_{2} \mathrm{O}_{3}(0001)$ films were prepared by a first oxidation treatment at $\mathrm{T}=1000 \mathrm{~K}$ in $10^{-1} \mathrm{mbar} \mathrm{O}_{2}$ and a final oxidation at $900-1000 \mathrm{~K}$ in $10^{-6}$ mbar $\mathrm{O}_{2}$. A single phase surface with a LEED pattern as in Fig. 5.3d was always observed under these conditions. For a biphase structure as shown in Fig. 5.34, higher temperatures are necessary. Although the final oxidation conditions differed slightly from the treatment described in Section $5.6(1120 \mathrm{~K}$ in $10^{-5} \mathrm{mbar}_{2}$ for the B-termination), we believe that the surface was B-terminated. After every single temperature ramp in TDS, the films were cleaned by heating to $\mathrm{T}=900 \mathrm{~K}$ in $10^{-6} \mathrm{mbar}_{2}$ in order to remove possible $\mathrm{C}$ deposits.

\subsection{Ethylbenzene adsorption}

\subsubsection{Thermal desorption spectroscopy}

\section{(i) TDS spectra}

Figure 6.1 shows TD scans for molecular desorption of EB after exposing the three Fe-oxide films to increasing amounts of EB. The inserts show the characteristic LEED patterns of the films. On the purely O-terminated surface of the $\mathrm{FeO}(111)$ monolayer film in Fig. 6.1a the initial desorption signal appears at $T=210 \mathrm{~K}$. With increasing exposure it shifts to $200 \mathrm{~K}$, until the signal becomes saturated at about $1.3 \mathrm{~L}$. We define this saturation coverage as $1 \mathrm{ML}$. The small shift to lower temperatures indicates a small repulsive adsorbate-adsorbate interaction and a first-order desorption kinetics. This weakly bound physisorbed species is denoted by $\beta$-EB. Upon further exposure, a new desorption signal at $T=155 \mathrm{~K}$ evolves. As shown in the insert this signal exhibits a common leading edge for all exposures, which is characteristic of a zero-order desorption kinetics. It does not become saturated upon further exposure and corresponds to condensed multilayers of EB denoted as $\alpha$-EB. 

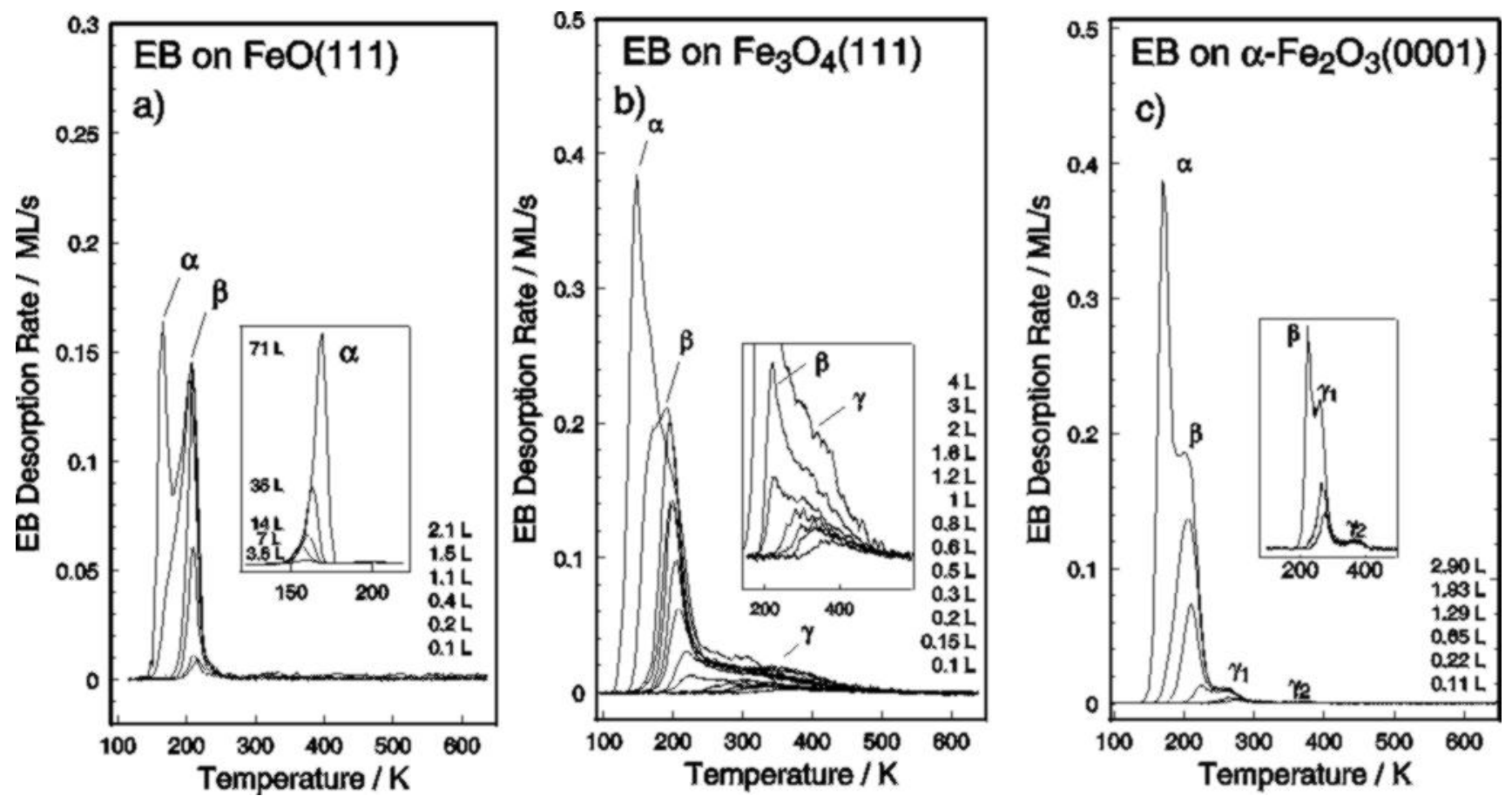

Fig. 6.1. Thermal desorption spectra of EB adsorbed on epitaxial Fe-oxide films grown onto Pt(111). (a) 1-2 ML thick $\mathrm{FeO}(111)$ film, (b) $\mathrm{Fe}_{3} \mathrm{O}_{4}(111)$ and (c) $\alpha-\mathrm{Fe}_{2} \mathrm{O}_{3}(0001)$ multilayer films. Exposures are given in Langmuir units $\left(1 \mathrm{~L}=1.33 \times 10^{-6} \mathrm{mbar} \cdot \mathrm{s}\right)$. LEED patterns of clean surfaces are shown in inserts. After [364, 365].

The TDS traces of EB adsorbed on the Fe-terminated surface of an $\mathrm{Fe}_{3} \mathrm{O}_{4}(111)$ multilayer film are shown in Fig. 6.1b. A desorption signal with a maximum around $T=400 \mathrm{~K}$ is observed for the lowest exposure, which corresponds to chemisorbed EB denoted by $\gamma$. With increasing exposure the peak maximum shifts to $250 \mathrm{~K}$ and a broad desorption signal is formed extending from 250 up to $450 \mathrm{~K}$. This chemisorbed $\gamma$-EB species becomes saturated at exposures between 0.5 and $0.8 \mathrm{~L}$, and a firstorder desorption kinetics is assumed for them. Upon further exposure, physisorbed $\beta$-EB appears, followed by the condensed $\alpha$-EB phase. The $\beta$ signal exhibits first-order desorption kinetics with a desorption maximum shifting from 210 to $190 \mathrm{~K}$, the $\alpha$ signal shows zero-order desorption kinetics and the same desorption temperatures as on the $\mathrm{FeO}(111)$ surface. Before the $\gamma$ signal becomes saturated, the $\beta$ peak already begins to form. We ascribe this deviation from sequential filling of adsorption sites to kinetic effects. The equilibrium occupation of $\gamma$ and $\beta$ sites is not established at the adsorption temperature of $100 \mathrm{~K}$ and not even during the heating ramp. The $\gamma$ and $\beta$ signals are saturated, when the $\alpha$-peak appears, which always occurs at exposures around $2 \mathrm{~L}$. This general appearence of the TDS spectra and the saturation of the $\gamma$ and $\beta$ signals around $2 \mathrm{~L}$ exposure is characteristic of all the $\mathrm{Fe}_{3} \mathrm{O}_{4}(111)$ films investigated. However, the intensity ratio between the $\gamma$ and $\beta$ signals varies from film to film, which is caused by different surface defect concentrations and surface morphologies. As deduced from the diffuse background intensities in the LEED patterns, which is a measure for the concentration of surface point defects, the $\gamma$ signal increases and the $\beta$ signal decreases with decreasing surface defect concentration. On surfaces with low defect densities more chemisorbed $\gamma$-EB is present. On the well-ordered $\mathrm{Fe}_{3} \mathrm{O}_{4}(111)$ film in Fig. 6.1b, the $\beta$-EB saturation coverage was the same as on the $\mathrm{FeO}(111)$ film, $\Theta_{\text {sat }}=1 \mathrm{ML}$. The saturation coverage of $\gamma$-EB was $0.8 \mathrm{ML}$, which is derived by integration of the respective contributions in the $2 \mathrm{~L}$ desorption trace. 
Figure 6.1c shows the TDS traces for EB on the B-terminated surface of an $\alpha-\mathrm{Fe}_{2} \mathrm{O}_{3}(0001)$ multilayer film. The inset shows that a molecular desorption signal labelled $\gamma_{2}$ appears at the beginning. It extends from 300 up to $450 \mathrm{~K}$ with its maximum located at around $400 \mathrm{~K}$. It always saturates at exposures around $0.1 \mathrm{~L}$. After that, a sharp desorption signal $\gamma_{1}$ appears with its maximum shifting from 275 to $250 \mathrm{~K}$. As on $\mathrm{Fe}_{3} \mathrm{O}_{4}(111)$, this $\gamma_{1}$ signal saturates at exposures between 0.5 and $0.8 \mathrm{~L}$. Together with the $\gamma_{2}$ signal, this results in a broad chemisorption signal extending from 250 up to $450 \mathrm{~K}$. However, in contrast to the $\mathrm{Fe}_{3} \mathrm{O}_{4}(111)$ surface, two clearly separated chemisorption states $\gamma_{1}$ and $\gamma_{2}$ are visible. As on $\mathrm{Fe}_{3} \mathrm{O}_{4}(111)$ and $\mathrm{FeO}(111)$, the physisorbed $\beta$ signal evolves upon further exposure. The the $\gamma$ and $\beta$ states are occupied almost sequentially. The desorption maximum of the $\beta$ signal shifts from 210 to $200 \mathrm{~K}$ with increasing exposure until it saturates at exposures around $2 \mathrm{~L}$. The $\alpha$ signal then starts to desorb at $155 \mathrm{~K}$, as on the $\mathrm{Fe}_{3} \mathrm{O}_{4}(111)$ surface. We assume first-order desorption kinetics for all the signals, except for the $\alpha$ signal, which shows zero-order desorption kinetics.

\section{(ii) Adsorption kinetics}

The adsorption kinetics of $\mathrm{EB}$ at the adsorption temperature of $100 \mathrm{~K}$ is illustrated by the uptake curves in Fig. 6.2. The EB coverage $\Theta$, determined from the area under the desorption traces, is plotted against the exposure $\varepsilon$ for the three surfaces investigated, not considering the condensed $\alpha$ species. On the $\mathrm{FeO}(111)$ surface the coverage increases linearly with exposure until it saturates at about $1.3 \mathrm{~L}$. This saturation coverage is defined as $1 \mathrm{ML}$. Since no strict sequential adsorption occurs for the $\beta$ and $\gamma$ states on the $\mathrm{Fe}_{3} \mathrm{O}_{4}(111)$ and $\alpha-\mathrm{Fe}_{2} \mathrm{O}_{3}(0001)$ surfaces, the sums of the $\beta+\gamma$ and $\beta+\gamma_{1}+\gamma_{2}$ peak areas are shown in Fig. 6.2. They also increase linearly until saturation is reached at exposures around $2 \mathrm{~L}$. Desorption of the condensed $\alpha$-species (onset marked by arrows) starts only after the $\beta+\gamma$ or $\beta+\gamma_{1}+\gamma_{2}$ phases have reached saturation. The $\beta$ and $\gamma_{1}$ peak areas were also determined separately (not shown here). They also increase fairly linearly with exposure, until saturation is reached, whereas the $\gamma_{2}$ peak area shows a non-linear increase for room temperature adsorption. On all films, the uptake curve for $\alpha$ EB (not shown) is linear without saturation, as expected for condensation.

The first derivatative of the uptake curves in Fig. 6.2 reveals the relative sticking coefficients $s / s_{0}=\mathrm{d} \Theta_{r} / \mathrm{d} \varepsilon$ as a function of the relative coverages $\Theta_{r}=\Theta_{x} / \Theta_{\text {sat }}$, where $x=\beta, \beta+\gamma$ and $\beta+\gamma_{1}+\gamma_{2}$ for the $\mathrm{FeO}(111), \mathrm{Fe}_{3} \mathrm{O}_{4}(111)$ and $\alpha-\mathrm{Fe}_{2} \mathrm{O}_{3}(0001)$ surfaces, respectively. These curves are plotted in Fig. 6.3. For all surfaces, the sticking coefficients are almost constant upto saturation. It is obvious that EB does not obey a Langmuir adsorption kinetics $s=s_{0}(1-\theta)$ (dotted line). The concave curves in Fig. 6.3 indicate a precursor mediated adsorption process. The adsorption kinetics via a mobile precursor was introduced by Kisliuk [106, 107] as discussed in Section 2.5. Within this model, the sticking coefficient is given by (2.17), and the fits represented by the solid lines in Fig. 6.3 yield a value of the Kisliuk constant $K$ between 0.06 and 0.02 . As shown in the next section, the sticking coefficient is near unity at the exposure temperature of $100 \mathrm{~K}$. This means that the probability of a precursor molecule on an empty site (intrinsic precursor) to transform into the chemisorbed state $p_{c h}^{\prime} \cong 1$ and the probability to desorb $p_{d}^{\prime} \cong 0$. The low observed value of $K$ is therefore due to a low desorption probability of an extrinsic precursor molecule $p_{d}^{\prime \prime} \cong 0.02-0.06$. The competing migration probability of the precursor on occupied sites must, thus, be $0.94-0.98$, which implies high mobility. 


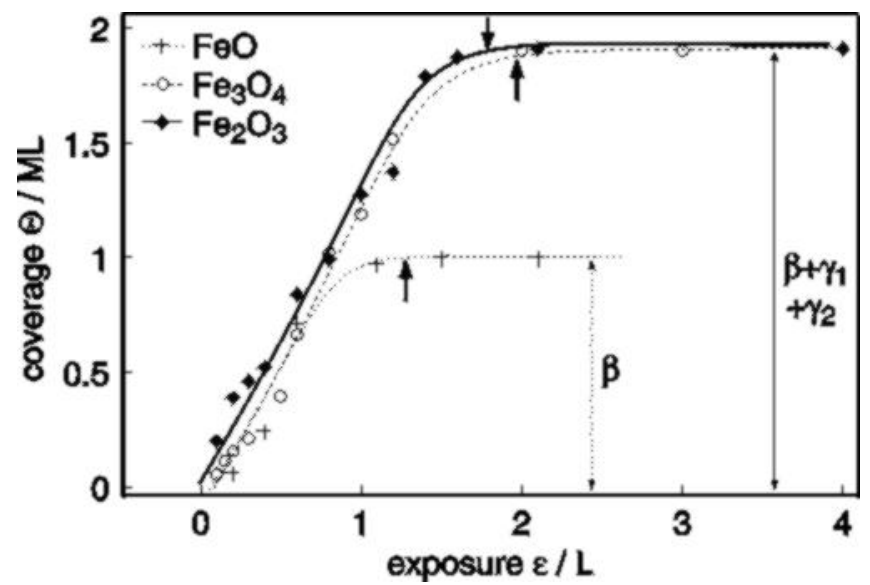

Fig. 6.2. Uptake curves coverage $\Theta$ versus exposure $\varepsilon$ for $\mathrm{EB}$ adsorbed onto $\mathrm{FeO}(111)$, $\mathrm{Fe}_{3} \mathrm{O}_{4}(111)$ and $\alpha-\mathrm{Fe}_{2} \mathrm{O}_{3}(0001)$ surfaces at $T=100$

$\mathrm{K}$. Coverage is evaluated from areas of corresponding TDS peaks. Saturation coverage of $\beta$-EB on $\mathrm{FeO}(111)$ is defined as $1 \mathrm{ML}$. Arrows indicate exposure where peak of condensed $\alpha$-EB starts to develop. After [235, 364].

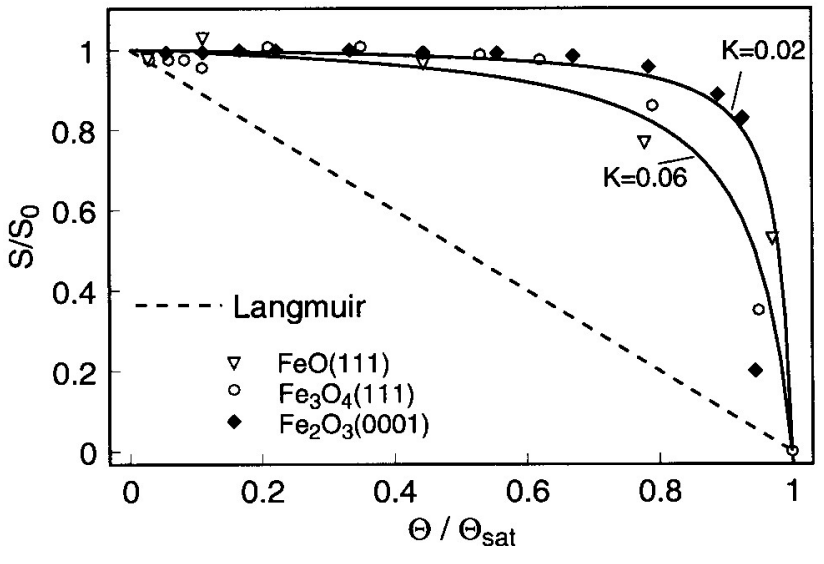

Fig. 6.3. Relative sticking coefficient $s / s_{0}$ vs. relative coverage $\theta / \theta_{\text {sat }}$ for data of Fig. 6.2, EB adsorption on $\mathrm{FeO}(111), \mathrm{Fe}_{3} \mathrm{O}_{4}(111)$ and $\alpha$ $\mathrm{Fe}_{2} \mathrm{O}_{3}(0001)$. Solid lines represent fits to data points according to Kisliuk model [106, 107] for an adsorption kinetics via mobile precursor states.

Dashed line indicates Langmuir like behaviour without mobile precursor states. From [235].

\section{(iii) Desorption energies and frequency factors}

For the condensed $\alpha$-EB signal with zero-order desorption kinetics the desorption energies $E_{d e s}$ and frequency factors $v$ were determined directly from the Polanyi-Wigner equation (2.7), because the desorption rate does not depend on coverage, in this case. The mean value is $E_{d e s}=44 \mathrm{~kJ} / \mathrm{mol}$, which agrees well with the heat of vaporization of $\mathrm{EB}$ of $42.8 \mathrm{~kJ} / \mathrm{mol}$ at room temperature [312]. The mean frequency factor is $v=5 \times 10^{8} \mathrm{~s}^{-1}$. Since a complete analysis was not possible with the available data, the leading edge analysis was used for determining the desorption energies and frequency factors of the other adsorption states.

Figure 6.4a shows the desorption energies of EB versus coverage for the three Fe-oxide films. On $\mathrm{FeO}(111)$, the desorption energy for $\beta$-EB is constant at $50-55 \mathrm{~kJ} / \mathrm{mol}$. Beyond $1 \mathrm{ML}$, it decreases abruptly to $44 \mathrm{~kJ} / \mathrm{mole}$, the value for condensed $\alpha$-EB. On $\mathrm{Fe}_{3} \mathrm{O}_{4}(111)$ the initial desorption energy of the $\gamma_{1}$ state is higher than $90 \mathrm{~kJ} / \mathrm{mol}$, and decreases very quickly with coverage to the value of physisorbed $\beta$-EB of $50-55 \mathrm{~kJ} / \mathrm{mole}$. This decrease is, in part, due to the sequential occupation of less strongly bound chemisorption states as the surface coverage increases, which causes the broadening of the $\gamma_{1}$ signal. However, repulsive lateral adsorbate-adsorbate interactions may also contribute to the decreasing desorption energy. As on $\mathrm{FeO}(111)$, the value for $\beta$-EB is constant $(50-55 \mathrm{~kJ} / \mathrm{mol})$. At about 2 ML coverage the desorption energy drops to the final value for $\alpha-E B$, which is the same as on $\mathrm{FeO}(111)$. As discussed above, the equilibrium distribution among $\beta$ - and $\gamma$-EB on $\mathrm{Fe}_{3} \mathrm{O}_{4}(111)$ is not established and the $\beta$ states start to become occupied long before the $\gamma$ states are saturated. Since desorption at the threshold is then dominated by $\beta$-EB desorption, the early drop of $E_{\text {des }}$ in Fig. 6.4 does not mark $\gamma$ saturation coverage. This is different for the transition from $\beta$ - to $\alpha$-desorption energies, because desorption of $\alpha$-EB does not start before saturation of $\beta+\gamma$. On the $\alpha-\mathrm{Fe}_{2} \mathrm{O}_{3}(0001)$ surface, the desorption maximum of the broad $\gamma_{2}$ signal reveals a desorption energy of about $94 \mathrm{~kJ} / \mathrm{mol}$, which then drops to the value for the $\gamma_{l}$ state of $64 \mathrm{~kJ} / \mathrm{mol}$. At about $0.5 \mathrm{ML}$ coverage the value of the 
$\beta$ state of $50-55 \mathrm{~kJ} / \mathrm{mol}$ is reached, which remains constant upto about $2 \mathrm{ML}$. The desorption energy then drops to the value for $\alpha-\mathrm{EB}$ of $44 \mathrm{~kJ} / \mathrm{mol}$. All values are listed in Table 6.4.
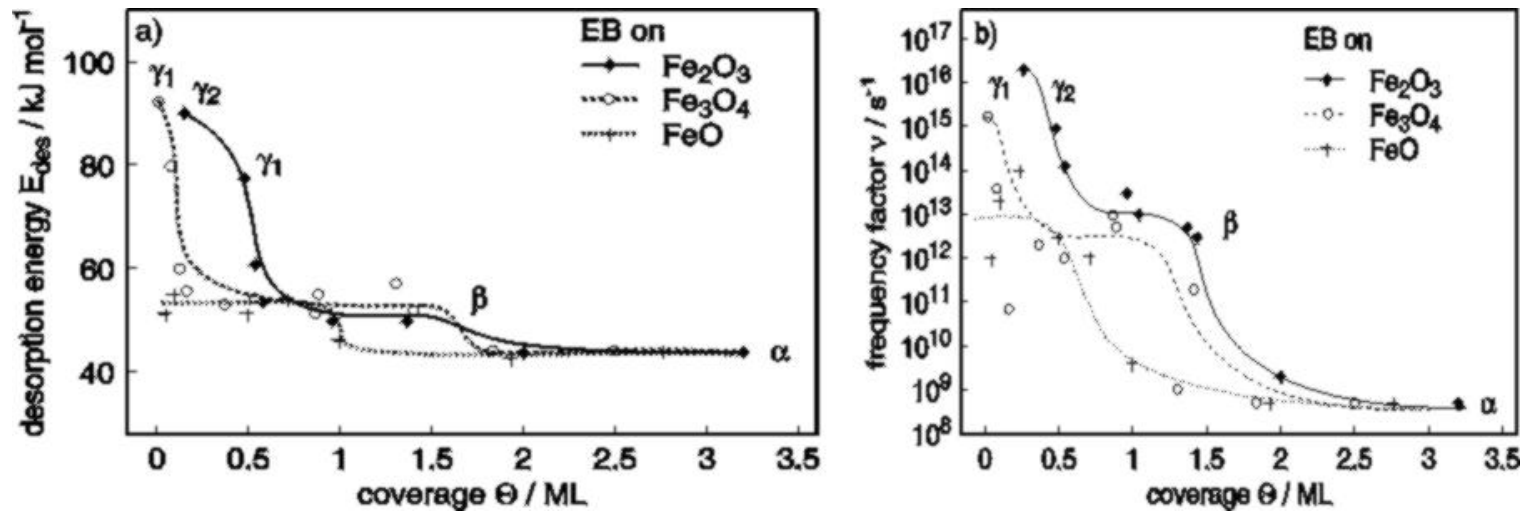

Fig. 6.4. Desorption energies $E_{\text {des }}$ (a) and preexponetial frequency factors $v$ (b) versus surface coverage $\theta$ for ethylbenzene adsorbed onto $\mathrm{FeO}(111), \mathrm{Fe}_{3} \mathrm{O}_{4}(111)$ and $\alpha-\mathrm{Fe}_{2} \mathrm{O}_{3}(0001)$ surfaces. After [235, 364].

The error of frequency prefactors determined by the leading edge method is high, which is the reason why the values for EB adsorption on the well-ordered films in Fig. 6.4b scatter strongly. The general shape, however, of the curves is similar to those of the desorption energies. This so-called compensation effect has been observed for many adsorption systems [129].

\subsubsection{UPS measurements - adsorbate identification and thermodynamics}

\section{(i) Adsorption-desorption equilibrium and coverage determination}

Figure 6.5 presents the set of UP spectra for EB adsorbed on $\mathrm{Fe}_{3} \mathrm{O}_{4}(111)$. Shown is the spectrum of the clean surface with no EB admission and the spectra measured under a constant EB partial pressure of $4 \times 10^{-9}$ mbar with the sample at temperatures between $300 \mathrm{~K}$ and $142 \mathrm{~K}$. Each spectrum represents adsorption-desorption (A-D) equilibrium at the respective temperature and is characterized by a certain coverage, a certain shape of the adsorbate spectrum and a certain work function change with respect to the clean surface. The latter is given by the shift of the low energy onset of the adsorbate covered spectrum with respect the spectrum of the clean surface. The spectra were taken after stepwise decrease of the temperature $T$. The adsorbate induced emission and, thus, the coverage increases with decreasing $T$. Stepwise increase of $T$, results in the same sequence of spectra over almost the whole coverage range, which proves that A-D equilibrium is generally established.

The adsorbate coverage was determined in two independent ways. The first method uses the attenuation of the substrate emission by the adsorbate overlayer. The attenuation factor is

$$
A F=I / I_{0}=\exp \left(-d / l_{e}\right) .
$$

$I_{0}$ and $I$ are the substrate intensities of the clean and of the covered surface, $d$ is the overlayer thickness and $l_{e}=\lambda_{e} \cos \theta$ the electron escape depth $\left(\lambda_{e}\right.$ is the mean free path and $\theta$ the mean escape angle of the photoelectrons with respect to the surface normal; $\theta=42^{\circ}$ for the CMA used here). The adsorbate thickness $d$ in units of the escape depth $l_{e}$ is then simply given by

$$
d / l_{e}=\ln (1 / A F)
$$

and is proportional to the coverage $\Theta$. The only region in the spectra without adsorbate emission is near the Fermi edge. The attenuation factor was adjusted, so that the difference spectra became zero at 
the positions marked by an arrow in Figs. 6.5 and 6.6. A problem may arise, if the substrate spectra are not only attenuated, but also changed in shape, due to the chemical interaction with the adsorbate. There are indications that this does happen. The effect is small, however, and the position of adjustment in the spectra has been selected to be affected as little as possible by this influence.

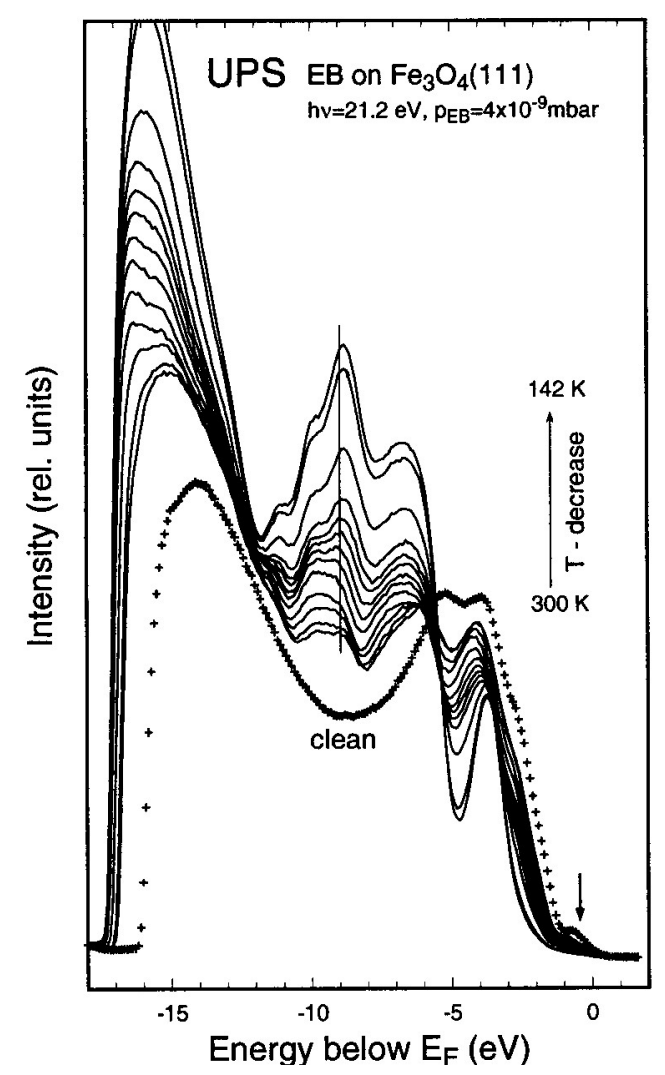

Fig. 6.5. He I excited UP spectra of clean epitaxial $\mathrm{Fe}_{3} \mathrm{O}_{4}(111)$ film and of $\mathrm{Fe}_{3} \mathrm{O}_{4}(111)$ surface in adsorption-desorption equilibrium with ethylbenzene at $p_{E B}=4 \times 10^{-9} \mathrm{mbar}$ at different sample temperatures between $300 \mathrm{~K}$ and $142 \mathrm{~K}$. Binding energy scale refers to Fermi energy $E_{F}$. From [71].

The second method uses the adsorbate induced valence-band emission intensity as a measure for $\Theta$. Difference spectra are formed by subtracting the properly attenuated clean substrate spectrum from the spectra of the adsorbate covered surface. Figure 6.6 shows these difference spectra for EB on $\mathrm{Fe}_{3} \mathrm{O}_{4}(111)$ and $\mathrm{FeO}(111)$. The secondary electron background is subtracted from the difference spectra using an empirical curve (crosses in Fig. 6.6a) that was adjusted to the spectra at -14 to $-15 \mathrm{eV}$ binding energies. The adsorbate-induced intensity $I_{E B}$ is then obtained by integration between -12 and $5 \mathrm{eV}$ binding energy. Since the highest lying molecular orbitals of chemisorbed EB are most strongly altered by the interaction with the substrate, they were excluded from integration. Beyond a coverage of $1 \mathrm{ML}, I_{E B}$ should no longer strictly increase in proportion to $\Theta$, because of attenuation of first adsorbate layer emission by the following layers.

Photoemission intensities are determined by the dipole matrix elements, which contain the initial and final states of the photoemission process [313]. These matrix elements and therefore the measured VB intensities may depend on the strength of adsorbate-substrate and adsorbate-adsorbate interaction. They depend further on the adsorbate orientation, which may change with $\Theta$. It has been shown, that $d / l_{e}$ and $I_{E B}$ are proportional to each other, as long as EB does not dissociate [71]. This proves that the adsorbate induced valence-band intensity, which can be measured easily and non-destructively, is a reliable measure for the adsorbate coverage. Another problem may originate from instrumental long term sensitivity changes during the measurements. Such changes influence the numerical values of $d / l_{e}$ and $I_{E B}$ in opposite directions. Let us assume that the sensitivity has increased, so that the intensity $I$ of the spectrum for the covered surface is higher than it should be compared to the clean surface reference 
spectrum. The integrated adsorbate intensity $\mathrm{I}_{\mathrm{EB}}$ and the coverage value deduced from it will then come out too high. Also, the attenuation factor AF in (6.2) will come out too large, but according to (6.3) this will result in a too small value for $d / l_{e}$, which can be used to correct the experimental data for sensitivity changes. It turned out that this was useful in the measurements for the determination of the isosteric heats of adsorption, which took up to six hours. Sensitivity changes upto $4 \%$ occurred within this period.
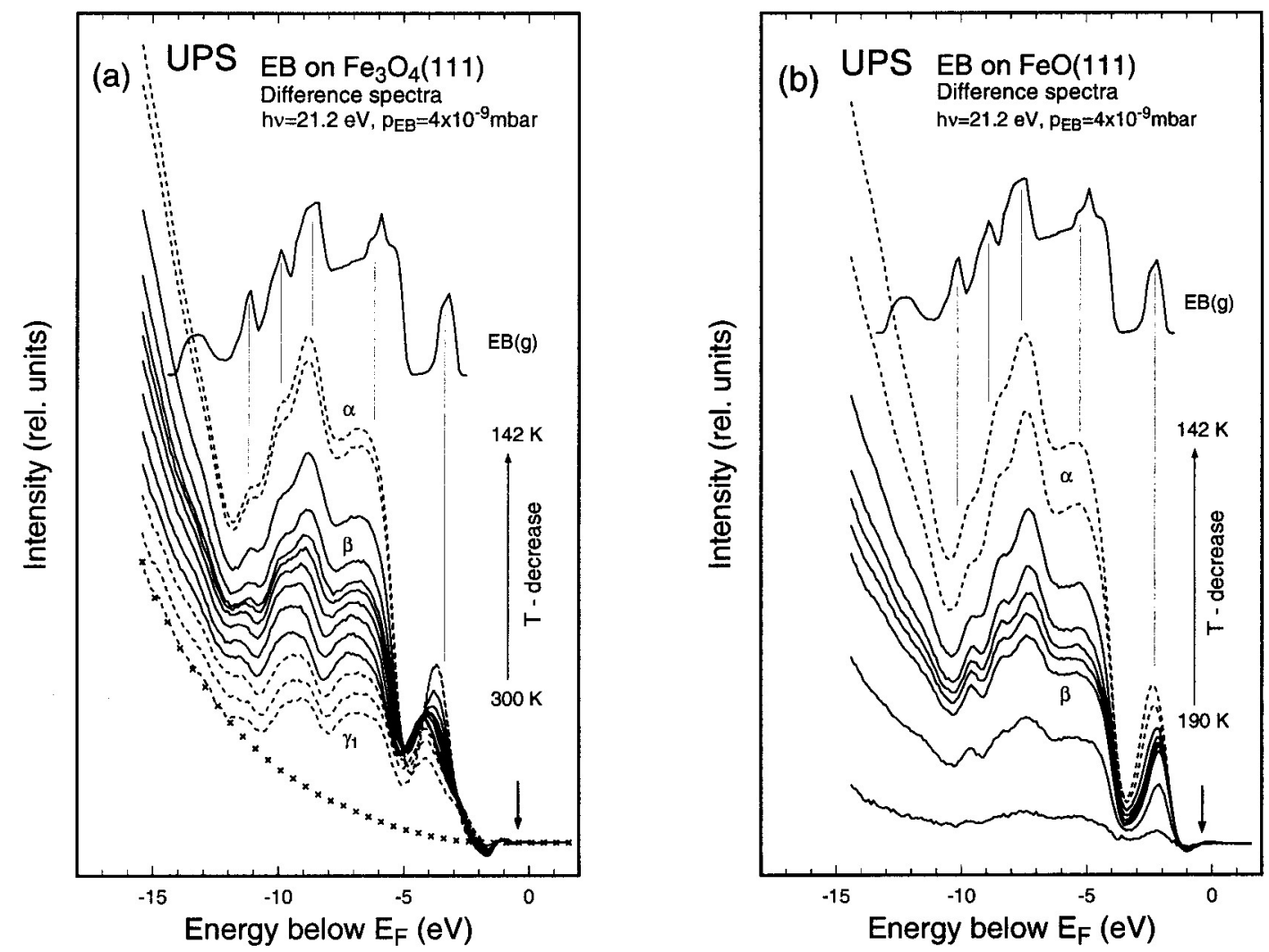

Fig. 6.6. Difference spectra between adsorbate covered and clean $\mathrm{Fe}_{3} \mathrm{O}_{4}(111)$ (a) and $\mathrm{FeO}(111)$ (b) surfaces, measured in ethylbenzene A-D equilibrium. Clean substrate spectra were attenuated to yield zero intensity at positions marked by arrows at $-0.4 \mathrm{eV} . \gamma$ marks spectra of chemisorbed $\mathrm{EB}, \beta$ marks physisorbed and $\alpha$ condensed $\mathrm{EB}$. On $\mathrm{FeO}(111)$ only $\beta$ - and $\alpha$-EB is observed. Crosses in (a) indicate used empirical background curve. Gas-phase spectrum (from [314]) is shifted to coincide with peaks of $\beta$-EB below $-9 \mathrm{eV}$ binding energy. From [71].

\section{(ii) Identification of adsorbed species}

Figure 6.6a presents difference spectra for $\mathrm{EB}$ adsorbed on $\mathrm{Fe}_{3} \mathrm{O}_{4}(111)$, measured in A-D equilibrium and obtained as described in the previous section. For comparison, the gas-phase spectrum $\mathrm{EB}(\mathrm{g})$ is shown. The spectra for the thick adsorbate layer are labelled $\alpha$. Because of their similarity to the gas-phase spectrum, we assign them to condensed molecular $\alpha$-EB. The spectra for intermediate coverages are labelled $\beta$. Their shape is the same as for $\alpha-E B$, but the temperature range of adsorption is higher. Therefore, they are assigned to physisorbed $\beta$-EB. The spectra for the lowest coverages labelled $\gamma$ show broader features than the spectra of $\alpha$-EB. The position of the highest lying peak, located at $-3.8 \mathrm{eV}$ for $\alpha$ - and $\beta$-EB, is shifted to $-4.3 \mathrm{eV}$ for $\gamma$-EB and a shoulder has formed at $-2.8 \mathrm{eV}$. We ascribe these changes to the interaction of EB with the substrate and assign $\gamma$-EB to molecularly 
chemisorbed EB. This is confirmed by the fact that it adsorbs already at $300 \mathrm{~K}$ and above. The spectra and their assignments are discussed in detail in Section 6.1.3.

Figure 6.6b shows the corresponding He I difference spectra for EB adsorbed onto a 1-2 ML thick $\mathrm{FeO}(111)$ film measured in A-D equilibrium. The most obvious difference to $\mathrm{Fe}_{3} \mathrm{O}_{4}(111)$ is the absence of a chemisorbed species. Adsorption of physisorbed $\beta$-EB starts only below $220 \mathrm{~K}$. From the early beginning of adsorption upto multilayer formation, the spectra are equal in shape and no shift or split of any molecular orbital feature is visible. Further, all orbitals seem to be shifted rigidly by about $1.5 \mathrm{eV}$ towards the right (lower $\mathrm{BE}$ ) when compared to $\mathrm{Fe}_{3} \mathrm{O}_{4}(111)$, which is due to the missing chemisorbate induced dipole on $\mathrm{FeO}(111)$, as discussed below.

\section{(iii) Adsorption kinetics and isosteric heats of adsorption}

Since all UP spectra of adsorbate covered surfaces were measured in A-D equilibrium, the plot of the adsorbate coverage in terms of $d / l_{e}$ or $I_{E B}$ versus $T$ yields adsorption isobars. The isobars for the $\mathrm{FeO}(111)$ and $\mathrm{Fe}_{3} \mathrm{O}_{4}(111)$ surfaces measured for three values of $p_{E B}$ are shown in Fig. 6.7. Even at $\mathrm{T}=360 \mathrm{~K}$, considerable amounts of $\mathrm{EB}$ are adsorbed on $\mathrm{Fe}_{3} \mathrm{O}_{4}(111)$. The UP spectra in this range correspond to chemisorbed $\gamma$-EB. The $\gamma$ coverage increases continuously with decreasing temperature. At $\mathrm{d} / \mathrm{l}_{\mathrm{e}} \approx 0.55$, the $\gamma$-species saturates and $\beta$-EB begins to adsorb on top of it. This can be deduced from the UP spectra in Fig. 6.6a, which start to change their shape (especially of the lowest BE peak) at this coverage. The steep increase below $170 \mathrm{~K}$ marks the beginning of $\alpha$-EB condensation. The isobars for EB adsorption on $\mathrm{FeO}(111)$ show no adsorption above $250 \mathrm{~K}$, since no chemisorbed species exists

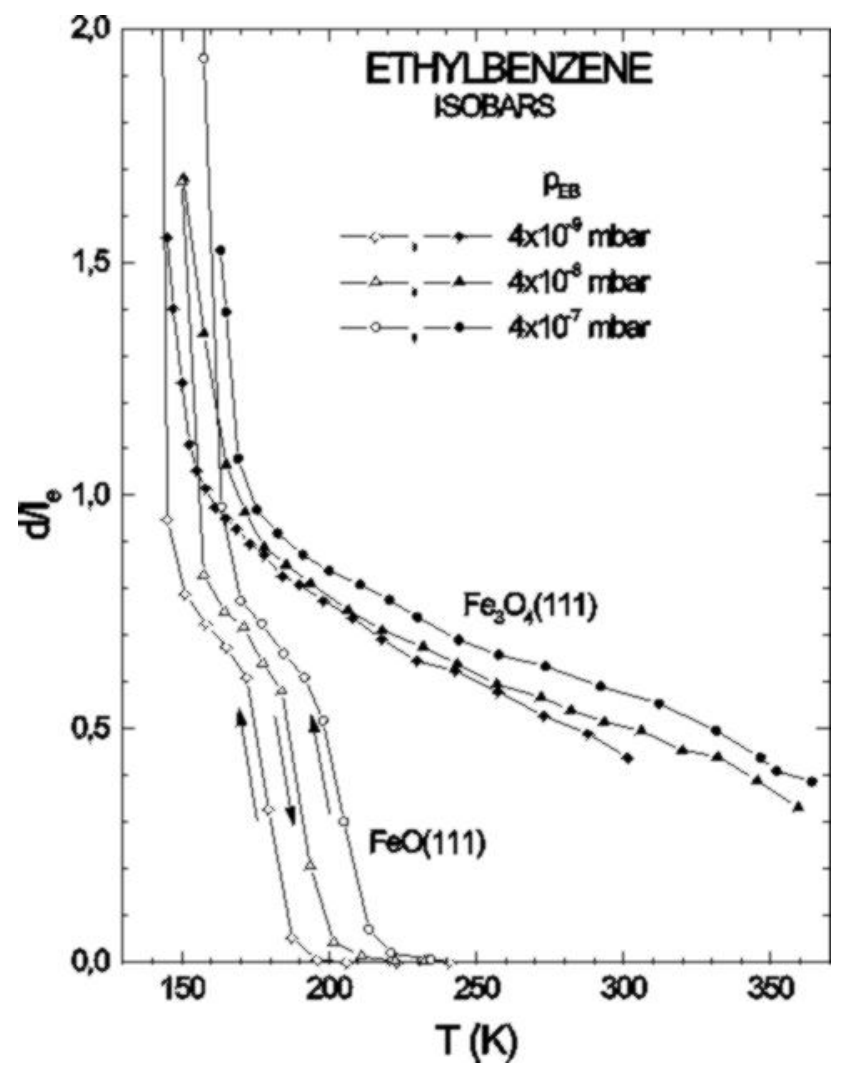

Fig. 6.7. Adsorption isobars coverage (in terms of $d / l_{e}$ ) versus temperature $T$ for adsorption of EB on $\mathrm{FeO}(111)$ and $\mathrm{Fe}_{3} \mathrm{O}_{4}(111)$ at different pressures as indicated. From [364]. 
here. Below $220 \mathrm{~K}$, the curves increase steeply with decreasing temperature, which is due to physisorption of $\beta$-EB as known from the UP spectra in Fig. 6.6b. A shoulder at $d / l_{e} \approx 0.7$ marks the saturation of the $\beta$-EB phase, followed by a further increase towards lower $T$, due to beginning condensation of $\alpha$-EB.

The three isobars for $\mathrm{FeO}(111)$ and $\mathrm{Fe}_{3} \mathrm{O}_{4}(111)$ in Fig. 6.7 are at three different EB pressures. They were measured in one run. Starting with the lowest EB pressure of $4 \times 10^{-9}$ mbar the sample was stepwise cooled down to $150 \mathrm{~K}$ and the UP spectra were taken, the EB pressure was then increased to $4 \times 10^{-8}$ mbar and spectra were measured during stepwise warming up. Finally, the pressure was set to $4 \times 10^{-7}$ mbar and another set of spectra were taken during cooling. The whole measurement took about 6 hours. For each coverage $\Theta$, three $p$ - $T$-pairs can be taken from the three isobars. According to the Clausius-Clapeyron equation, as discussed in Sections 2.2 and 2.4, the isosteric heat of adsorption $q_{s t}(\Theta)$ can be deduced from the slope of the Arrhenuis plots $\ln p$ over 1/T, which are shown in Fig. 6.8. In general, the three data points for each coverage fit well to a straight line. Only in the $\beta$-EB region on $\mathrm{Fe}_{3} \mathrm{O}_{4}(111)$ do deviations occur, which are due to non-equilibrium between the $\alpha$ - and $\beta$-EB phases, as
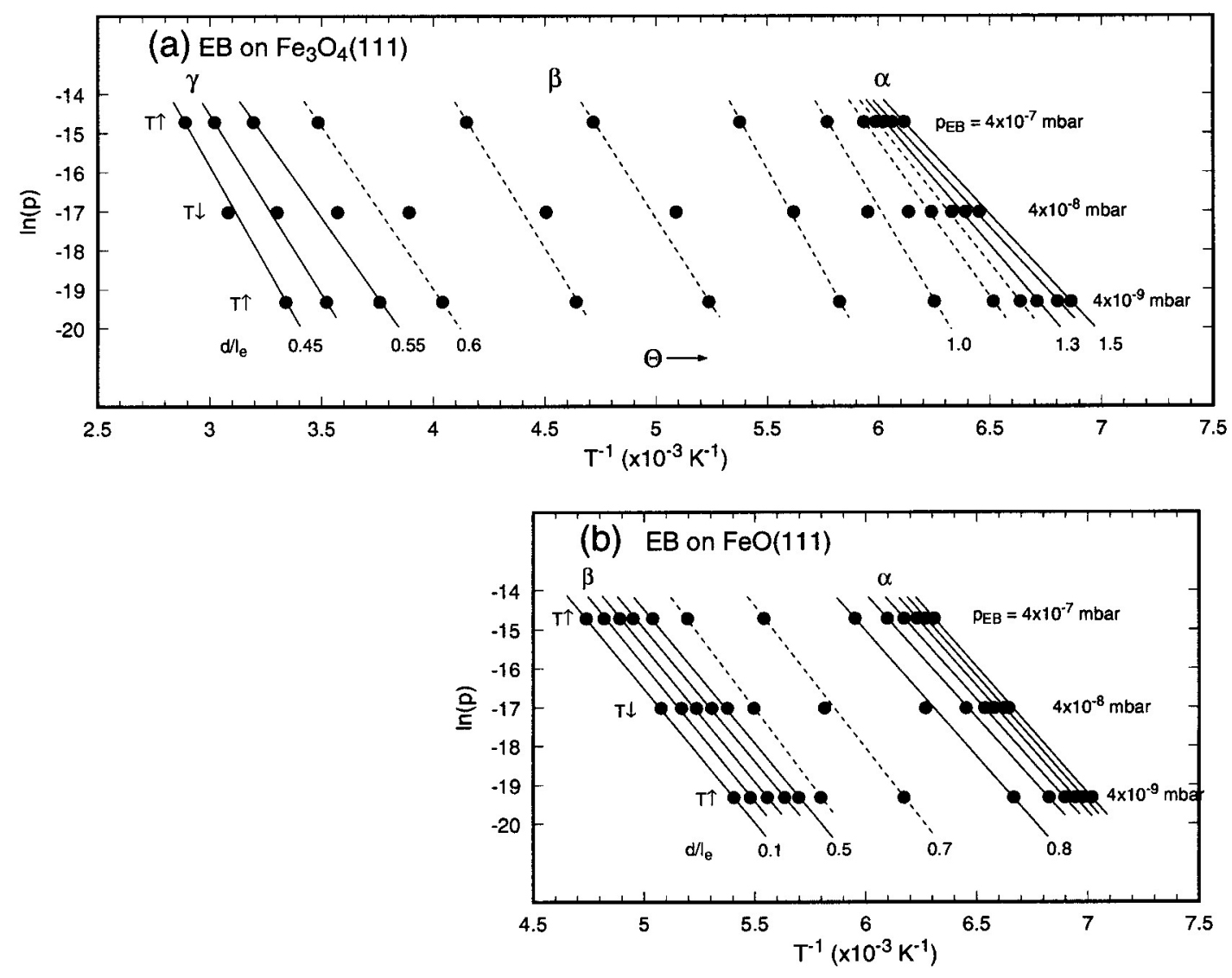

Fig. 6.8. Arrhenius plots $\ln p$ over $T^{-1}$ for different coverages (in terms of $d / l_{e}$ ) as deduced from adsorption isobars in Fig. 6.7. (a) $\mathrm{EB}$ on $\mathrm{Fe}_{3} \mathrm{O}_{4}(111)$; (b) $\mathrm{EB}$ on $\mathrm{FeO}(111)$.

discussed in the next section. Strong changes in the offset along the $1 / T$ axis occur in the transition regions between adsorption of different species, especially for EB adsorption on $\mathrm{FeO}(111)$ in Fig. 6.8b. The reason for these strong offset changes are changes in the adsorption kinetics. As derived in section 2.4, the plots in Fig. 6.8 are given by (2.15) 


$$
\ln p=\ln \left[\frac{\Theta}{1-\Theta} N_{A} \sqrt{2 \pi m k T_{\text {gas }}} \frac{v(\Theta)}{s^{*}}\right]-\frac{q_{s t}(\Theta)}{k T},
$$

which is formally equal to the Clausius-Clapeyron equation (2.6). The logarithmic offset term in brackets depends on coverage $\Theta$ and on the adsorption kinetics via the ratio of the preexponetial factors for desorption $v$ and adsorption $s^{*}$. The $\Theta / 1-\Theta$ function varies strongly only for $\Theta \rightarrow 0$, i.e. at the beginning of adsorption of a new species on top of an already saturated one. For a new species, new preexponential factors may also change the A-D kinetics. Therefore, the strong offset changes in the Arrhenius plots within small coverage ranges are due to changes in the A-D kinetics occuring when one adsorbate species is saturated and the adsorption of the next one on top of it begins.

The deduced isosteric heats of adsorption $q_{s t}(\Theta)$ for $\mathrm{EB}$ on $\mathrm{FeO}(111)$ and $\mathrm{Fe}_{3} \mathrm{O}_{4}(111)$ are shown in Fig. 6.9b. On $\mathrm{FeO}(111)$, there are two regions with slightly different values for $q_{s t}$, which correspond to physisorbed $\beta$-EB $\left(q_{s t}=58 \mathrm{~kJ} / \mathrm{mol}\right)$ and condensed $\alpha$-EB $\left(q_{s t}=52 \mathrm{~kJ} / \mathrm{mol}\right)$. The heat of adsorption for $\beta$-EB is constant within the error limits up to $\mathrm{d} / \mathrm{l}_{\mathrm{e}}=0.7$, then it drops to the lower value for $\alpha$-EB. This coverage range also corresponds to the transition between the $\beta$ and $\alpha$ ranges in Fig. 6.8a and is assigned to a closed ML of physisorbed $\beta$-EB. If we assume that EB is lying flat on the surface with the van der Waals thickness of benzene ( $d=3.7 \AA$ [315] ), we obtain (using (6.2)) a value for the electron mean free path $\lambda_{e}=6.5 \AA$ for the energy $E \approx 20 \mathrm{eV}$ above the valence-band edge, which is a reasonable value and compares well with tabulated data [316]. In Section 6.1.4, we shown that EB at saturation is actually not adsorbed flat, but at an average angle of $40^{\circ}$ with respect to the surface. This would formally alter the monolayer thickness slightly. All coverage scales in terms of monolayers are based on this calibration. On $\mathrm{Fe}_{3} \mathrm{O}_{4}(111), q_{s t}$ is about $85 \mathrm{~kJ} / \mathrm{mol}$ for chemisorbed $\gamma$-EB at low coverages and decreases to $65 \mathrm{~kJ} / \mathrm{mol}$ at $d / l_{e}=0.55$, which corresponds to the saturation coverage of $\gamma$-EB $(\Theta=0.8$ ML). The decrease of $q_{s t}$ with increasing coverage indicates a repulsive adsorbate-adsorbate interaction. Above this coverage, the $q_{s t}$ values for $\mathrm{Fe}_{3} \mathrm{O}_{4}(111)$ in Fig. 6.9b scatter and assume quite high values. This unexpected behaviour is also evident from the adsorption isobars in Fig. 6.7, which are not equidistant for $d / l_{e}$ values between 0.5 and about 1.1. The reason for this is a non-equilibrium between the $\beta$ - and $\alpha$-EB phases, which is discussed below. Therefore, the $q_{s t}$ values in this range are not reliable. The UP spectra indicate that the adsorbed species in this range is physisorbed $\beta$-EB. Beyond $d / l_{e}=1.25, q_{s t}$ reaches the final value for condensed $\mathrm{EB}(52 \mathrm{~kJ} / \mathrm{mol})$. On the $\mathrm{Fe}_{3} \mathrm{O}_{4}(111)$ surface, the thickness of the $\beta$-EB layer, as deduced from the width on the coverage scale $\Delta\left(d / l_{e}\right)=0.7$, also corresponds to about $1 \mathrm{ML}$, as on $\mathrm{FeO}(111)$.

Figure 6.9a compares the desorption energies $E_{\text {des }}$ for $\mathrm{EB}$ on $\mathrm{FeO}(111)$ and $\mathrm{Fe}_{3} \mathrm{O}_{4}(111)$ determined from the TDS spectra in Fig. 6.1 with the isosteric heats of adsorption. They generally agree well. The desorption energies for condensed $\alpha$-EB deduced from TDS are about $10 \mathrm{~kJ} / \mathrm{mol}$ smaller than the isosteric heats of adsorption deduced from UPS. This may be caused by the lower adsorption temperature $(100 \mathrm{~K})$ in the TDS measurements, where condensed EB might form a less well-ordered structure than during the UPS measurements under A-D equilibrium. Since in the UPS measurements performed under A-D equilibrium a sequential filling of the states takes place, the $q_{s t}$ values of the $\gamma$ species decrease at higher coverages, when compared to the TDS data, as discussed above. To establish equilibrium coverages below $\Theta=0.5 \mathrm{ML}$, sample temperatures above $400 \mathrm{~K}$ would be required. UP spectra in this range revealed a slow decomposition of EB. Therefore, no values of $q_{s t}$ could be determined in this coverage range. 

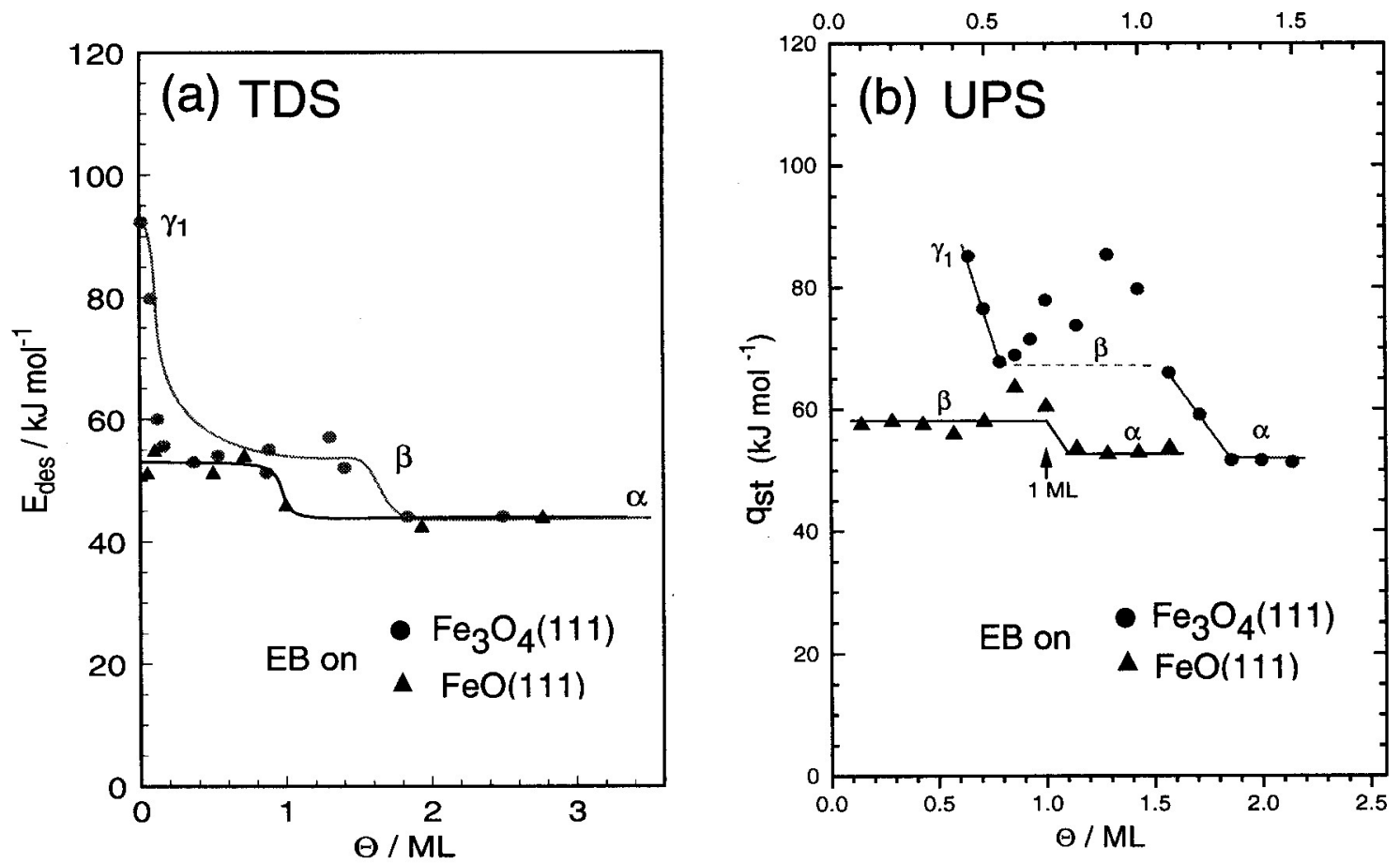

Fig. 6.9. (a) Coverage dependence of desorption energy $E_{\text {des }}$ for $\mathrm{EB}$ on $\mathrm{FeO}(111)$ and $\mathrm{Fe}_{3} \mathrm{O}_{4}(111)$ determined from TDS data in Fig. 6.1. (b) Coverage dependence of isosteric heat of adsorption $q_{s t}$ for

$\mathrm{EB}$ on $\mathrm{FeO}(111)$ and $\mathrm{Fe}_{3} \mathrm{O}_{4}(111)$ determined from $\mathrm{EB}$ adsorption isobars in Fig. 6.7 according to Clausius- Clapeyron equation. From [364].

\subsubsection{Electronic structure of adsorbates}

(i) Work function change

The work function $\varphi$ of a system with electronic states filled up to the Fermi edge (metallic) can be determined from the resolution-corrected total width $W$ of its photoelectron spectrum and is simply given by

$$
\varphi=h v-W .
$$

The work function $\varphi$ of the clean $\mathrm{FeO}(111)$ film is $\varphi_{\mathrm{FeO}(111)}=5.90 \pm 0.05 \mathrm{eV}$ [71]. This quite high value is in agreement with the purely O-terminated surface structure of this film. The $\mathrm{Fe}_{3} \mathrm{O}_{4}(111)$ surface is terminated by $1 / 4 \mathrm{ML}$ of $\mathrm{Fe}$ ions in the topmost layer, and a smaller work function $\varphi_{\mathrm{Fe} 3 \mathrm{O} 4(111)}=5.52 \pm 0.05 \mathrm{eV}$ is observed. EB adsorption greatly decreases the work function, as can be seen from the shift of the low energy onset in the UP spectra in Fig. 6.5. The work function change $\Delta \varphi$ is plotted versus the $\mathrm{EB}$ coverage $\Theta$ or $d / l_{e}$ in Fig. 6.10. On $\mathrm{FeO}(111), \Delta \varphi$ decreases by $0.05-0.1 \mathrm{eV}$ at quite small coverages. It cannot be ruled out that this is due to adsorption of EB onto small $\operatorname{Pt}(111)$ substrate regions that were not covered by Fe-oxide. After that, $\Delta \varphi$ decreases slowly and reaches -0.25 $\mathrm{eV}$ for saturation of the physisorbed $\beta$-EB layer at $1 \mathrm{ML}$ coverage. The formation of the condensed layer is connected with a further decrease down to a final value of $-0.53 \mathrm{eV}$.

The work function change induced by chemisorbed $\gamma$-EB on $\mathrm{Fe}_{3} \mathrm{O}_{4}$ is linear and quite strong. The arrows indicate the direction of the measurement. The open circles are measured upon stepwise decrease of $T$ (increasing coverage), the full circles upon stepwise increase of $T$ (decreasing coverage). For the $\gamma$-EB saturation coverage $(\Theta=0.8 \mathrm{ML}) \Delta \varphi=-1.0 \mathrm{eV}$. Using the Helmhotz-equation

$$
\Delta \varphi=n \mu / \varepsilon_{0},
$$


the dipole moment $\mu$ per adsorbed molecule can be calculated. The van der Waals area of a flat lying EB molecule is about $50 \AA^{2}$. Assuming adsorption in a flat lying configuration, the monolayer density would be $n_{M L}=2 \times 10^{14} \mathrm{~cm}^{-2}$. For $\Theta=0.8 \mathrm{ML}$, the adsorbate density is $n_{M L}=1.6 \times 10^{14} \mathrm{~cm}^{-2}$, and one obtains $\mu_{\gamma}=1.62 \mathrm{D}$ (for comparison: the dipole moment of the free water molecule is $1.84 \mathrm{D}$ ). This high value indicates strong polarization of $\gamma$-EB and is comparable with a value of $2 \mathrm{D}$ for benzene adsorbed on transition-metal surfaces [129].

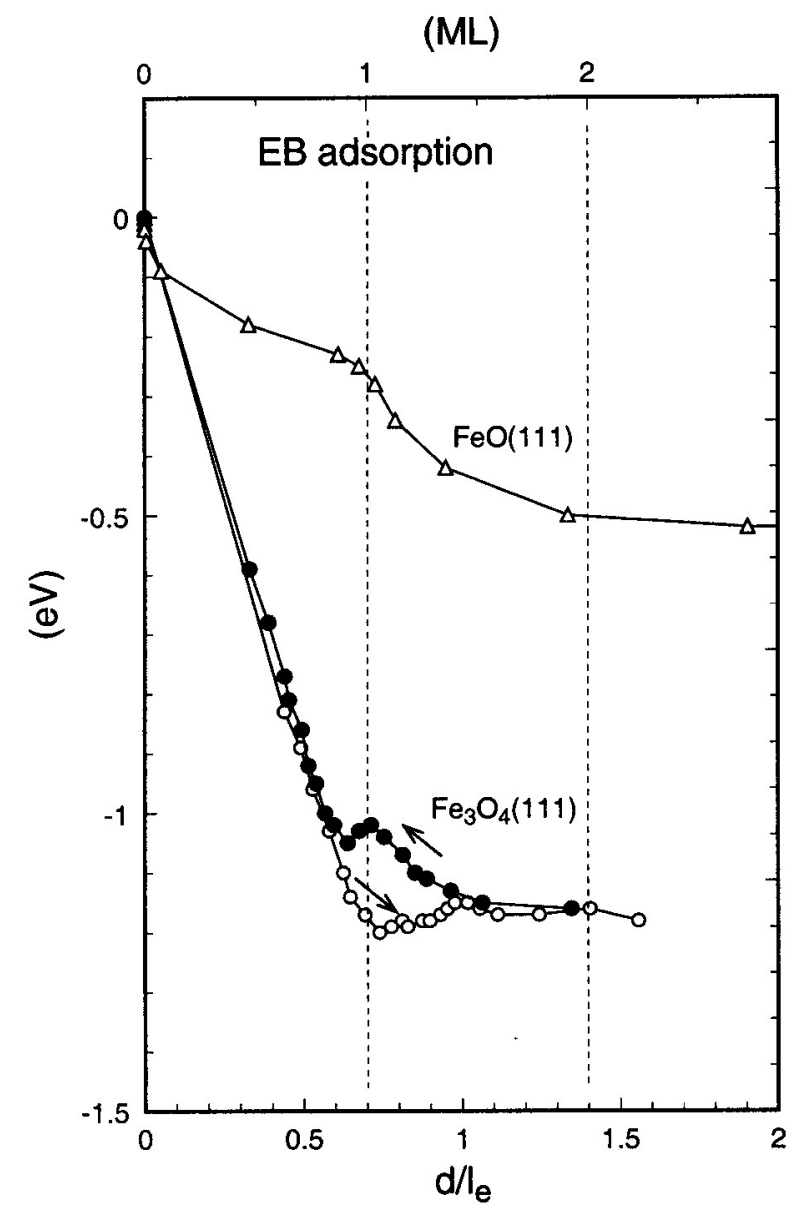

Fig. 6.10. (Left). Work function change $\Delta \varphi$ as function of EB coverage in terms of $d / l_{e}$ and ML for $\mathrm{EB}$ adsorption on $\mathrm{FeO}(111)$ and $\mathrm{Fe}_{3} \mathrm{O}_{4}(111)$.

$\mathrm{On} \mathrm{Fe}_{3} \mathrm{O}_{4}(111), \Delta \varphi$ depends on direction of coverage change in range $0.55<d / l_{e}<1.0$ and forms a hysteresis loop. From [71].

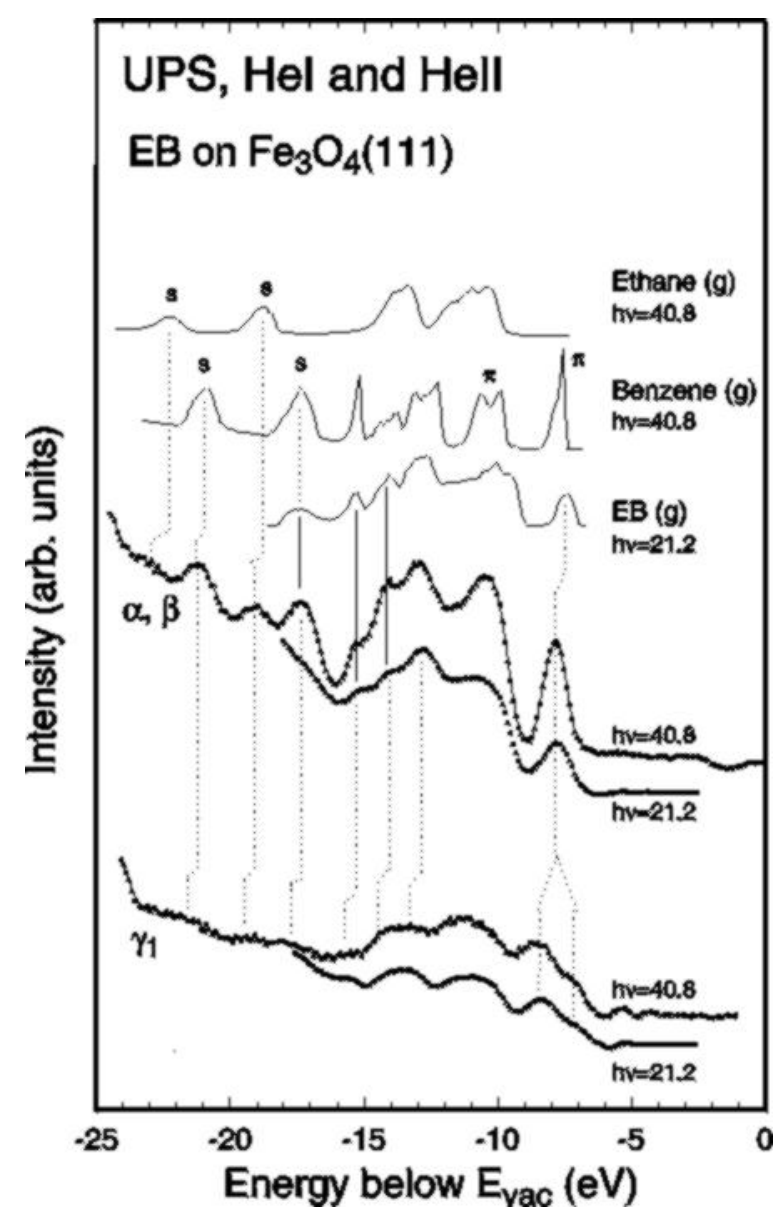

Fig. 6.11. (Right). He I and He II spectra of chemisorbed $\gamma$-EB and condensed $\alpha$-EB on $\mathrm{Fe}_{3} \mathrm{O}_{4}(111)$. They are compared to He I excited gas-phase spectrum of EB (from [314]) and with He II excited gas-phase spectra of benzene [317] and ethane [318]. $\pi$-orbitals are marked $\pi$. Orbitals labeled "s" are preferentially C2s-derived $\sigma$ orbitals. In order to adjust gas-phase spectra to adsorbate spectra in lower valence-band region (solid lines) relaxation shift $\Delta E_{R}=2.3 \mathrm{eV}$ towards right was applied. After [71].

Beyond $\gamma$ saturation, the $\Delta \varphi$ curve depends on the direction of the measurement and forms a hysteresis loop, which is interpreted as follows: With increasing coverage, first the chemisorbed $\gamma$ layer is formed, on top of it the physisorbed $\beta$ and finally the condensed $\alpha$ layer. When the condensed layer 
reaches a certain thickness, the physisorbed layer underneath transforms and adjusts its structure to the condensed layer. This starts probably around $\Theta=1 \mathrm{ML}$, where the $T \downarrow$ measurement reaches its minimum. The transformation is finished around $\Theta=1.4 \mathrm{ML}$, where the $T \downarrow$ and the $T \uparrow$ curves coincide. During the $T \uparrow$ measurement, the condensed layer continues to desorb below $\Theta=1.4$ ML since the backtransformation to $\beta$-EB is kinetically hindered. Only near $d / l_{e}=0.7$ (1 ML) does the rest of $\alpha$-EB transform back to physisorbed $\beta$-EB. Since the heat of adsorption for the condensed species is smaller, desorption proceeds "too fast" in the range $\Theta=1.4$ - $1 \mathrm{ML}$, which also results in the too low lying $4 \times 10^{-8}$ mbar isobar in Fig. 6.7. The coverage in this range is, thus, strongly influenced by the transformation kinetics between $\alpha$ - and $\beta$-EB and no equilibrium coverages are established. Consequently, the $q_{s t}$ values are unreliable.

\section{(ii) Analysis of adsorbate spectra}

In general, spectral features of adsorbed molecules are shifted towards smaller binding energies, i.e., towards the vacuum level, when compared with gas-phase spectra. This extra-atomic relaxation shift $\Delta E_{R}$ [319] originates from the screening of the hole generated during the ionization process by the surrounding condensed phase. Chemical interaction with the substrate may induce an additional bonding shift $\Delta E_{B}$ of the involved orbitals, usually towards higher BE. If the adsorbate symmetry is strongly affected, energetically degenerate orbitals may split or rearrange.

In Fig. $6.11 \mathrm{He}$ I and $\mathrm{He}$ II spectra of $\alpha$ - and $\gamma$-EB on $\mathrm{Fe}_{3} \mathrm{O}_{4}(111)$ are compared to the gas-phase spectra of EB [314], benzene [317, 320] and ethane [318]. The EB spectrum has strong similarities with the sum of the benzene and ethane spectra. The features between -9 and $-16 \mathrm{eV}$ result from overlapping contributions of the phenyl and the ethyl group but the lower lying peaks in the spectrum of adsorbed EB are well separated and can easily be assigned. The peaks at $-17.3 \mathrm{eV}$ and $-21.2 \mathrm{eV}$ correspond to $\mathrm{C} 2 \mathrm{~s}$ derived $\sigma$-orbitals of benzene and, thus, originate from the phenyl ring, whereas the peaks at $-19.0 \mathrm{eV}$ and $-23.0 \mathrm{eV}$ originate from $\mathrm{C} 2 \mathrm{~s}$ derived $\sigma$-orbitals of the ethyl group. Also the relative intensities agree with this assignment. The vacuum level $E_{V A C}$ was chosen as reference level for the adsorbate spectra. Compared to the spectra in Figs. 6.5 and 6.6 the eneergy axis is shifted by the work function values. The gas-phase spectra were adjusted by $\Delta E_{R}$ to the adsorbate spectra in the lower valence-band region at -13 to $-18 \mathrm{eV}$. The peaks in this range originate from $\sigma$-orbitals formed from relatively strongly localized $\mathrm{C} 2 \mathrm{~s}$ electrons and we expect a negligible bonding shift. The corresponding relaxation shifts $\Delta E_{R}$ of the different adsorbed species are listed in Table 6.1.

The spectra of $\alpha$ - and $\beta$-EB look equal both on $\mathrm{FeO}(111)$ and $\mathrm{Fe}_{3} \mathrm{O}_{4}(111)$ and are only shifted with respect to each other by small differences in the relaxation energies $(\leq 0.2 \mathrm{eV}$, see Table 6.2$)$. The relaxation energies are larger than the value observed for condensed benzene layers (1.0-1.4 eV [321323]). Representative of them, Fig. 6.11 shows the spectra of $\alpha$-EB adsorbed on $\mathrm{Fe}_{3} \mathrm{O}_{4}(111)$. The peaks of $\alpha$-EB at -7.8 and at $-10.4 \mathrm{eV}$ are shifted additionally by about $0.4 \mathrm{eV}$ towards higher BE with respect to the gas-phase spectrum. The $-7.8 \mathrm{eV}$ peak corresponds to the highest lying $\pi$-orbitals of the phenyl ring, which are degenerate in benzene $\left(\mathrm{e}_{1 \mathrm{~g}}\right.$ orbitals [324]). In EB, the degeneracy is lifted, but the split is too small to be resolved in the spectra. The lowest lying $\pi$-orbital of the phenyl group is expected on the low energy side of the $-10.4 \mathrm{eV}$ peak. We attribute the shift of the center of gravity of this peak with respect to the gas-phase EB spectrum to a shift of this $\pi$-orbital, too. The direction of these extra shifts of the $\Delta E_{\pi}$ is opposite to that of the relaxation shifts, and they result from bonding interactions between the $\pi$-orbitals in the condensed phase. For condensed benzene layers, such interactions have not been observed by photoemission spectroscopy [321, 322].

Apart from an additional peak broadening of the phenyl derived $\pi$-orbitals, the spectrum of the $\gamma$ species is similar to that of $\alpha$-EB, indicating a molecular chemisorption of EB. The centers of gravity 
of the lower lying orbitals (below $-12.5 \mathrm{eV}$ ) are shifted by about $0.4 \mathrm{eV}$ towards the left, indicating a reduced relaxation shift of $1.9 \mathrm{eV}$ in the first layer, when compared to $\Delta E_{R}=2.3 \mathrm{eV}$ and $2.5 \mathrm{eV}$ in the second layer and multilayer, respectively. The highly polarizable $\pi$-electrons in the condensed and physisorbed EB phases induce a stronger screening of the photoelectron hole than the ionic $\mathrm{Fe}_{3} \mathrm{O}_{4}$ surface. The strongest differences occur in the $\pi$-orbitals: The highest lying $\pi$-orbital is split into two components located -0.6 and $+0.8 \mathrm{eV}$ with respect to the corresponding signal in $\alpha$-EB, and the maximum of the peak near $-11 \mathrm{eV}$ (lowest lying phenyl derived $\pi$-orbital) is shifted by about $-0.5 \mathrm{eV}$. These changes indicate a strong chemical interaction between the $\pi$-orbitals in the phenyl ring and the oxide surface.

Similar extra bonding shifts for the $\pi$-orbitals of benzene chemisorbed on a variety of surfaces [321] [325-327], were taken as indication that the molecule is adsorbed with the plane of the ring parallel to the surface. In the next section, it is demonstrated that EB at low coverage really is adsorbed with the phenyl ring lying flat on the surface. At saturation, however, it is tilted. Nevertheless, the observed bonding shift of the $\pi$-orbitals shows that the interaction still occurs via the $\pi$-orbitals. Moreover, the observed large dipole moment of $\mu_{\gamma}=1.62 \mathrm{D}$ agrees with the strong polarizbility of the extended $\pi$ orbitals. The highest observed saturation coverage for $\gamma$-EB is $0.8 \mathrm{ML}$, which is clearly less than the saturation coverage of the physisorbed layer $(1 \mathrm{ML})$. In the previous section, it was shown that $0.8 \mathrm{ML}$ correspond to density of $1.6 \times 10^{14} \mathrm{~cm}^{-2}$. The density of a complete Fe layer is $3.3 \times 10^{14} \mathrm{~cm}^{-2}$, which is exactly twice as much. It is concluded that each EB molecule occupies two Fe sites at saturation. Defective surfaces have lower saturation coverages. As discussed in Section 5.5, the most frequent type of defects are Fe deficiencies, which shows that chemisorption occurs on regular surface areas of $\mathrm{Fe}_{3} \mathrm{O}_{4}(111)$ and that a specific interaction with the exposed Fe cations is involved. The split of the topmost $\pi$-orbital of chemisorbed EB suggests a symmetry breaking of the molecule, because of deformations of the ethyl group and/or phenyl ring, caused by the interaction with the substrate. Deformations and expanded $\mathrm{C}-\mathrm{C}$ bond distances were observed for benzene adsorbed on several transition metal surfaces [129]. On Pt(111), the benzene molecule adsorbs on bridge sites and assumes a "boat-like" shape [328], accompanied by a change from a sixfold to a twofold local symmetry. For benzene on $\mathrm{Si}(001)$, the specific interaction between the $\pi$-electrons and the dangling bonds result into even stronger modifications of the molecule geometry in the same direction, leading to a splitting of several levels in the UP spectra $[329,330]$.

\subsubsection{NEXAFS measurements}

Figure 6.12 shows NEXAFS spectra of the C K-edge for condensed EB, St, benzene and octane. In the spectrum of condensed EB and St, the spectral features A', A-E are marked. Their assignment in Table 6.1 is based on the analogy to the data for benzene and octane, which were analysed before [331, 332]. The spectra of EB and St are similar to the spectrum of benzene. The resonance A is slightly broader for EB and St than for benzene, which is ascribed to symmetry breaking by the side chains. The styrene resonance has a low energy shoulder $\mathrm{A}^{\prime}$, which can be assigned to the $\pi^{*}$-states of the vinyl group [333]. The Rydberg resonance B has a slightly higher intensity for EB and St due to additional contributions from the side chain $\mathrm{C}$ atoms. The spectra of the condensed phases were also measured at normal and glancing incidence (not shown). They are almost independent of the incidence angle, indicating an average tilt angle of $54^{\circ}$, which is also consistent with a random orientation [250].

Figure 6.13 presents $\mathrm{C} \mathrm{K}$ edge NEXAFS spectra for low EB coverages, corresponding to physisorbed $\beta$-EB on $\mathrm{FeO}(111)$ and to chemisorbed $\gamma$-EB on $\mathrm{Fe}_{3} \mathrm{O}_{4}(111)$ and $\alpha-\mathrm{Fe}_{2} \mathrm{O}_{3}(0001)$. They are compared to the multilayer spectrum. All energetic positions of the resonances agree with those of the condensed phase. The first $\pi^{*}$ resonance $(\mathrm{A})$ of condensed EB is narrow (FWHM $=0.7 \mathrm{eV}$ ). Also, for the weakly interacting $\beta$-EB on $\mathrm{FeO}(111)$ it remains narrow $(\mathrm{FWHM}=0.9 \mathrm{eV}$ ), but for the 
chemisorbed $\gamma$-species on $\mathrm{Fe}_{3} \mathrm{O}_{4}(111)$ and $\alpha$ - $\mathrm{Fe}_{2} \mathrm{O}_{3}(0001)$ it is broadened considerably $(\mathrm{FWHM}=1.2$ $\mathrm{eV})$. The $\pi^{*}$ resonances exhibit a strong dichroism with higher intensity for grazing incidence. It is much stronger for the chemisorbed species. Assuming undisturbed electronic structure of the adsorbates, the tilt angle between the plane of the phenyl ring and the surface (or between the direction of the dipole transition moment and the surface normal) can be calculated from this dichroism. This analysis yields tilt angles of $39 \pm 2^{\circ}$ on $\mathrm{FeO}(111), 7^{\circ} \pm 2^{\circ}$ on $\mathrm{Fe}_{3} \mathrm{O}_{4}(111)$ and $21^{\circ} \pm 2^{\circ}$ on $\alpha-\mathrm{Fe}_{2} \mathrm{O}_{3}(0001)$. The $\sigma^{*}$ resonances (D, E) show the opposite intensity behaviour as expected from theory [250]. When the coverage is increased, the tilt angle for the chemisorbed species increases to $40^{\circ}$ for saturation (Fig. 6.14).

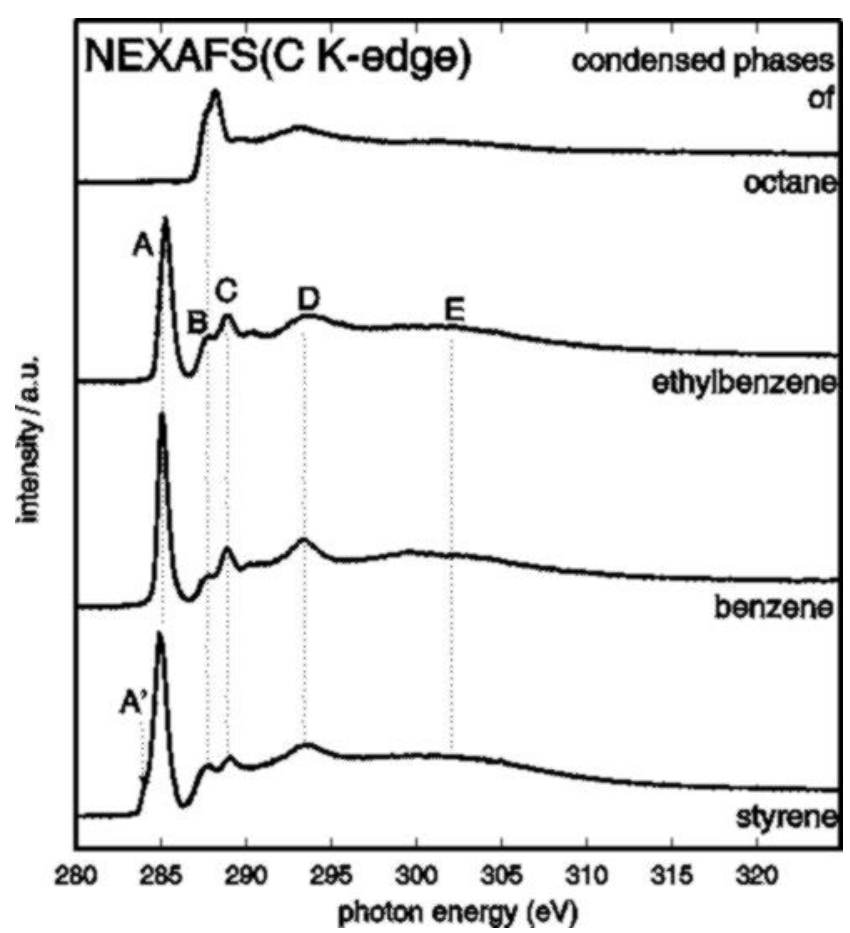

Fig. 6.12. (Left). Comparison of C K-edge NEXAFS spectra of condensed phases of styrene, benzene, ethylbenzene and octane. Assignment of resonances $\mathrm{A}^{\prime}$ and $\mathrm{A}-\mathrm{E}$ is given in table 6.1. From [358].

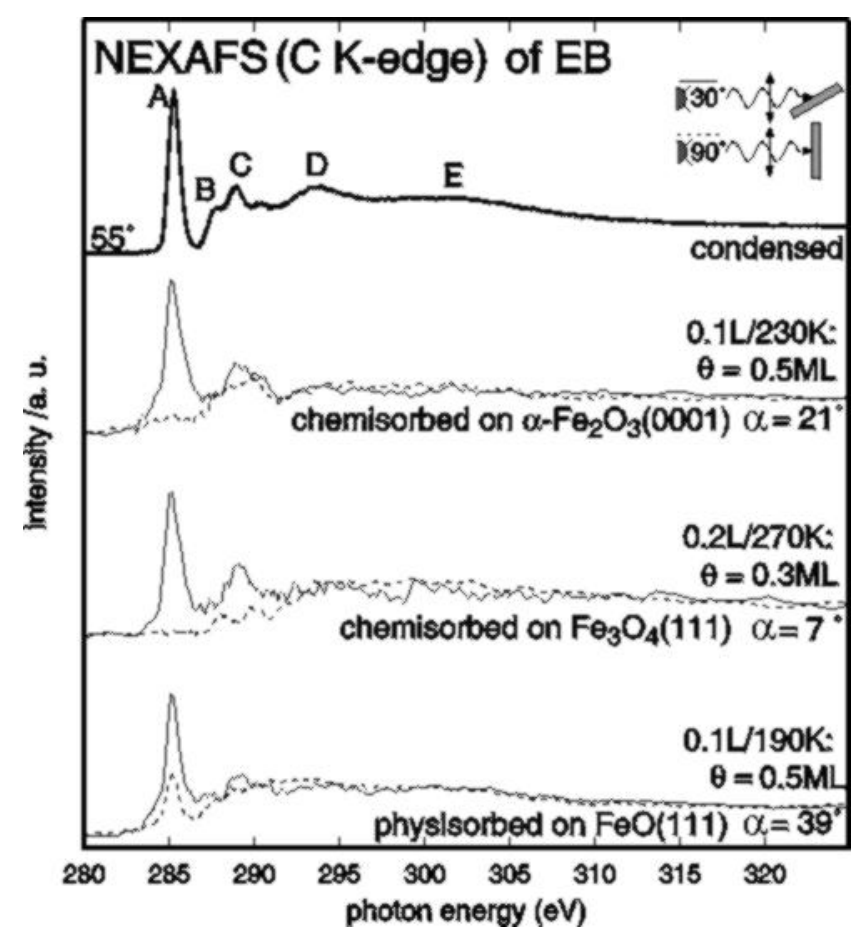

Fig. 6.13. (Right). C K-edge NEXAFS spectra of ethylbenzene layers on different Fe-oxides in submonolayer regime. Full lines: grazing incidence $\left(30^{\circ}\right)$; dashed: normal incidence $\left(90^{\circ}\right)$. Exposure conditions (doses and temperatures), resulting coverages $(\theta)$ and calculated tilt angles between molecular plane and surfaces $(\alpha)$ are indicated. From [358]. 
TABLE 6.1. Energetic positions and assignments of NEXAFS resonances observed in Figs. 6.12 6.14 .

\begin{tabular}{|c|c|c|}
\hline Resonance & Energetic position $(\mathrm{eV})$ & Assignment \\
\hline $\mathrm{A}^{\prime}$ (only styrene) & 284.1 & $\pi^{*}$, vinyl group \\
\hline $\mathrm{A}$ & 285.0 & $\pi^{*}$, ring (benzene: $\mathrm{C} 1 \mathrm{~s} \rightarrow \mathrm{e}_{2 \mathrm{u}}$ ) \\
\hline \multirow{2}{*}{$\mathrm{B}$} & 286.9 & Rydberg, side chain (octane) \\
\cline { 2 - 3 } & 287.6 & Rydberg, ring (benzene) \\
\cline { 2 - 3 } & 288.0 & Rydberg, side chain (octane) \\
\hline $\mathrm{C}$ & 288.9 & $\pi^{*}$, ring (benzene: $\left.\mathrm{C} 1 \mathrm{~s} \rightarrow \mathrm{b}_{2 \mathrm{~g}}\right)$ \\
\hline $\mathrm{D}$ & 293.1 & $\sigma^{*}$, side chain $($ octane) \\
\cline { 2 - 3 } & 293.3 & $\sigma^{*}$, ring (benzene: $\left.\mathrm{C} 1 \mathrm{~s} \rightarrow \mathrm{e}_{1 \mathrm{u}}\right)$ \\
\hline \multirow{2}{*}{$\mathrm{E}$} & 300.2 & $\sigma^{*}$, ring (benzene: $\mathrm{C} 1 \mathrm{~s} \rightarrow \mathrm{e}_{1 \mathrm{u}}+\mathrm{a}_{2 \mathrm{~g}}$ ) \\
\cline { 2 - 3 } & 301.0 & $\sigma^{*}$, side chain $($ octane $)$ \\
\hline
\end{tabular}

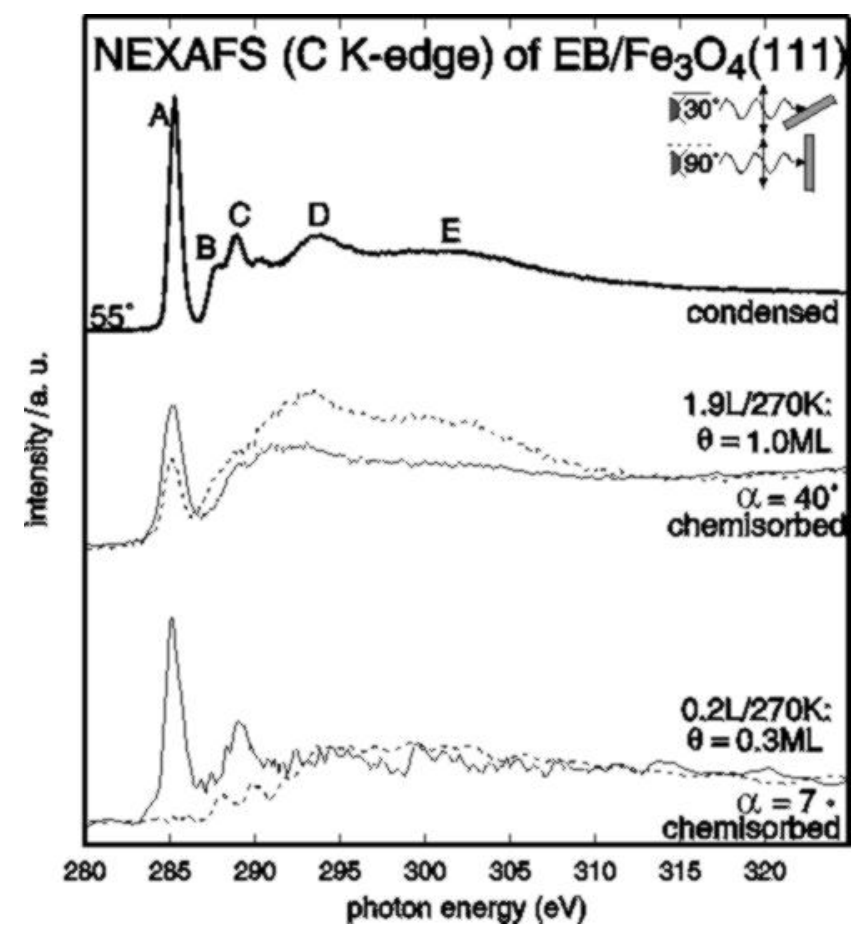

Fig. 6.14. (Left). Coverage dependence of C Kedge NEXAFS spectra for EB chemisorbed on $\mathrm{Fe}_{3} \mathrm{O}_{4}(111)$. Full lines: grazing incidence $\left(30^{\circ}\right)$; dashed: normal incidence $\left(90^{\circ}\right)$. Exposure conditions (doses and temperatures), resulting coverages $(\theta)$ and calculated tilt angles between molecular plane and surface $(\alpha)$ are indicated.

From [358].

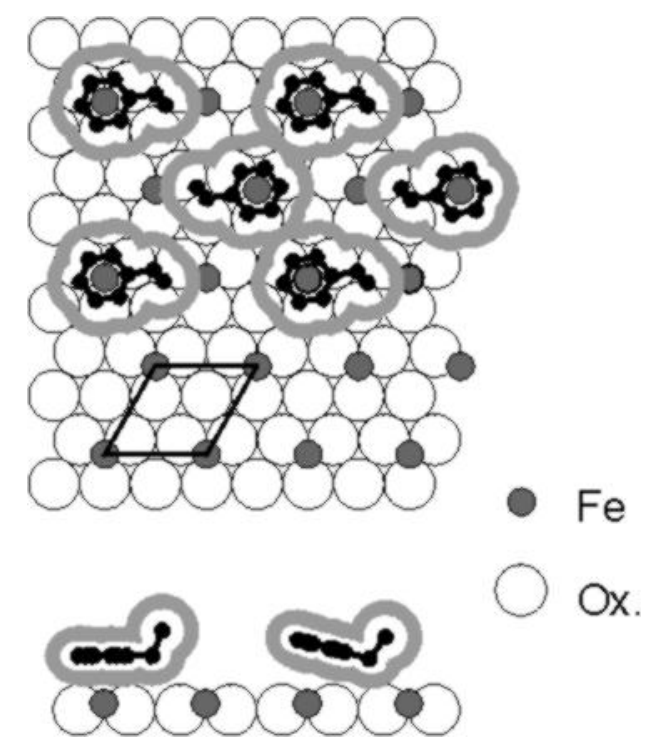

Fig. 6.15. (Right). Model for ethylbenzene chemisorbed on $\mathrm{Fe}_{3} \mathrm{O}_{4}(111)$ surface in top view (left) and side view (right). Hexagonal surface unit cell of $\mathrm{Fe}_{3} \mathrm{O}_{4}(111)$ is indicated. Lower part in top view shows clean substrate surface. Thick shaded lines represent van der Waals contour of EB.

\subsubsection{Discussion}

On the Fe-terminated $\mathrm{Fe}_{3} \mathrm{O}_{4}(111)$ surface the first layer of $\mathrm{EB}$ is chemisorbed with a maximum coverage of $0.8 \mathrm{ML}$, followed by physisorption on top of the chemisorbed layer and finally condensation. On $\alpha-\mathrm{Fe}_{2} \mathrm{O}_{3}(0001)$, the sequence of species is the same. Chemisorption takes place on 
regular surface areas and is not related to surface defects. On the purely O-terminated $\mathrm{FeO}(111)$ surface, a chemisorbed state is missing and only physisorbed and condensed EB are formed. This indicates that the chemisorption reactivity is related to the Fe cations exposed on the $\mathrm{Fe}_{3} \mathrm{O}_{4}(111)$ surfaces, whereas such sites are absent on the chemically inert $\mathrm{FeO}(111)$ surface. Further, the chemisoption on $\alpha-\mathrm{Fe}_{2} \mathrm{O}_{3}(0001)$ is related to the existence of states derived from second layer $\mathrm{Fe}$ atoms, even if the surface is O-terminated or hydroxylated. The EB molecule is initially chemisorbed with the aromatic phenyl ring lying almost flat on the surface, and it is strongly polarized with the negative charge towards the surface. The shift and split of the $\pi$-levels of EB chemisorbed on $\mathrm{Fe}_{3} \mathrm{O}_{4}(111)$, the strong work function change upon chemisorption, the broadening of the $\pi^{*}$-resonances for chemisorption on $\mathrm{Fe}_{3} \mathrm{O}_{4}$ and $\alpha-\mathrm{Fe}_{2} \mathrm{O}_{3}$ show that the chemical interaction takes place between the $\pi$ electrons of the aromatic ring and the Fe cations. The chemisorption geometry with the phenyl ring initially parallel to the surface suggests a $\eta^{6}$-like hapto complex of the Fe cation with the aromatic ring of $\mathrm{EB}$ or St. Although the molecules are tilted at higher coverage, the saturation of $\mathrm{EB}$ on $\mathrm{Fe}_{3} \mathrm{O}_{4}$ at only $0.8 \mathrm{ML}$ still suggests a quantitative relationship (two Fe sites per molecule), maybe in a tilted $\eta^{6}$ like complex. The $\pi$-electron system acts as a Lewis base, being polarized by interaction with the Lewis acidic Fe cations. A deformation of the EB molecule and changed $\mathrm{C}-\mathrm{C}$ bond distances are likely. Only a few investigations for benzene adsorbed on metal-oxide surfaces exist. Flat lying and a slighty tilted $\left(\approx 10^{\circ}\right)$ configurations were reported on the non-polar (1 $\left.\overline{1} 00\right)$ [325] and (10 $\left.\overline{1} 0\right)$ [334, 335] surfaces of $\mathrm{ZnO}$, respectively. Near parallel and tilted configurations were reported on the (100)$(1 \times 1)$ and (100)-(1×3) surfaces of $\mathrm{TiO}_{2}$ [336]. In none of these studies was the chemical interaction as strong as here, leading to a splitting of the highest lying $\pi$-orbital. This might be caused by the more exposed $\mathrm{Fe}$ atoms in the topmost layer of $\mathrm{Fe}_{3} \mathrm{O}_{4}(111)$, when compared to the other oxides. On both the $\alpha-\mathrm{Fe}_{2} \mathrm{O}_{3}(0001)$ and $\mathrm{Fe}_{3} \mathrm{O}_{4}(111)$ surfaces chemisorbed $\mathrm{EB}$ desorbs below $450 \mathrm{~K}$. However, two distinct chemisorption states $\gamma_{1}$ and $\gamma_{2}$ are observed on $\alpha-\mathrm{Fe}_{2} \mathrm{O}_{3}(0001)$, whereas a broad distribution of chemisorbed $\gamma$-EB species is observed on $\mathrm{Fe}_{3} \mathrm{O}_{4}(111)$.

The desorption energies $E_{\text {des }}$ determined by TDS agree well with the corresponding isosteric heats of adsorption $q_{s t}$, determined by UPS under adsorption-desorption equilibrium conditions, indicating non-activated adsorption for EB. The values for the different adsorption states and their coverage ranges are listed in Table 6.2, together with their relaxation shifts $\Delta E_{R}$ and $\pi$-orbital binding energy shifts $\Delta E_{\pi}$ observed in the UPS measurements. On all the surfaces the adsorption at $T=100 \mathrm{~K}$ occurs via weakly bound mobile precursor states, except for the $\gamma_{2}$ state on $\alpha-\mathrm{Fe}_{2} \mathrm{O}_{3}(0001)$, which shows a different adsorption kinetics at room temperature. As deduced from the exposures, where saturation of the first layers is observed, the sticking coefficients are close to unity for these adsorption states at $T=100 \mathrm{~K}$. EB-uptake curves measured by UPS (not shown here) also indicate such high sticking coefficients for room temperature adsorption.

Figure 6.15 presents a model for $\mathrm{EB}$ chemisorbed on $\mathrm{Fe}_{3} \mathrm{O}_{4}(111)$. The phenyl rings are assumed to be located on top of Fe cations ( $\eta^{6}$-complex). The shown saturation coverage corresponds to $0.8 \mathrm{ML}$ and it is evident that a higher coverage with all molecules in this coordination is not possible. For lower coverage, the EB molecules do not touch and may lie flat on the surface. Beyond half of the saturation coverage, they unavoidable move closer to each other than the van-der-Waals contour allows. The resulting repulsive interaction reduces the BE (Fig. 6.9b) and causes tilting. Even when tilted, the $\eta^{6}$-configuration may be retained, as schematically shown in the side view. The EB molecules are shown with the ethyl group orientation, as in the gas-phase. A change of its bending angle in the chemisorbed state may occur. The arrangement would give rise to a superstructure in LEED, which was not observed. It is very likely that a considerable rotational and translational mobility prevents long-range order. A similar arrangement is expected for EB chemisorbed on $\alpha$ $\mathrm{Fe}_{2} \mathrm{O}_{3}(0001)$, as the NEXAFS measurements revealed a similar transition from flat to tilted adsorption. 
The TDS mesurements showed that the main species $\gamma_{l}$ is bound more weakly, which suggest even stronger disorder.

TABLE 6.2. Coverage ranges (saturation of $\beta$-EB on $\mathrm{FeO}(111)$ corresponds to $1 \mathrm{ML}$ ), relaxation shifts $\Delta E_{R}$, and binding energy shifts of highest lying $\pi$-orbitals with respect to $\mathrm{EB}$ (gas) $\Delta E_{\pi}$ for $\mathrm{EB}$ adsorbed on $\mathrm{FeO}(111), \mathrm{Fe}_{3} \mathrm{O}_{4}(111)$ and $\mathrm{Fe}_{2} \mathrm{O}_{3}(111)$ surfaces. $\Delta E_{\pi}<0$ means increase of binding energy towards left in Fig. 6.11.

\begin{tabular}{|c|c|c|c|c|}
\hline Substrate & $\begin{array}{c}\text { Adsorbate } \\
\text { species }\end{array}$ & $\begin{array}{c}\text { Coverage } \\
\text { range } \Theta\end{array}$ & $\begin{array}{c}\Delta E_{R} \\
(\mathrm{eV})\end{array}$ & $\begin{array}{c}\Delta E_{\pi} \\
(\mathrm{eV})\end{array}$ \\
\hline $\mathrm{FeO}(111)$ & $\beta$-EB (phys.) & $0-1 \mathrm{ML}$ & 2.5 & -0.4 \\
\hline $\mathrm{FeO}(111)$ & $\alpha$-EB (cond.) & $>1 \mathrm{ML}$ & 2.5 & -0.4 \\
\hline $\mathrm{Fe}_{3} \mathrm{O}_{4}(111)$ & $\gamma$-EB (chem.) & $0-0.8 \mathrm{ML}$ & 1.9 & $-1.0,+0.4$ \\
\hline $\mathrm{Fe}_{3} \mathrm{O}_{4}(111)$ & $\beta$-EB (phys.) & $0.8-2.0 \mathrm{ML}$ & 2.3 & -0.4 \\
\hline $\mathrm{Fe}_{3} \mathrm{O}_{4}(111)$ & $\alpha$-EB (cond.) & $>2 \mathrm{ML}$ & 2.3 & -0.4 \\
\hline$\alpha-\mathrm{Fe}_{2} \mathrm{O}_{3}(0001)$ & $\gamma 2$-EB (chem.) & $\approx 0-0.1 \mathrm{ML}$ & - & - \\
\hline$\alpha-\mathrm{Fe}_{2} \mathrm{O}_{3}(0001)$ & $\gamma$-EB (chem.) & $\approx 0.1-0.8 \mathrm{ML}$ & - & - \\
\hline$\alpha-\mathrm{Fe}_{2} \mathrm{O}_{3}(0001)$ & $\beta$-EB (phys.) & $0.8-2.0 \mathrm{ML}$ & - & - \\
\hline$\alpha-\mathrm{Fe}_{2} \mathrm{O}_{3}(0001)$ & $\alpha$-EB (cond.) & $>2 \mathrm{ML}$ & - & - \\
\hline
\end{tabular}

${ }^{1}$ two levels observed

\subsection{Water adsorption}

\subsubsection{Thermal desorption spectroscopy}

Figure 6.16 shows TDS traces of water adsorbed on the three Fe-oxide films. As for EB, chemisorbed $\gamma$, physisorbed $\beta$ and condensed $\alpha$-species are adsorb sequentially on the Fe-terminated $\mathrm{Fe}_{3} \mathrm{O}_{4}$ and on the $\alpha-\mathrm{Fe}_{2} \mathrm{O}_{3}$ multilayer films, whereas only physisorbed $\beta$ and condensed $\alpha$-species are adsorbed on the O-terminated $\mathrm{FeO}$ film. The physisorbed $\beta$-species on $\mathrm{FeO}$ desorbs initially $T=170 \mathrm{~K}$. With increasing coverage, the desorption maximum shifts to slightly lower temperatures $(165 \mathrm{~K}$ at 0.6 L) before condensation ( $\alpha$-species) starts. Condensation is characterized by by the common leading edge of the following desorption traces. On the $\mathrm{Fe}_{3} \mathrm{O}_{4}$ multilayer film, a chemisorbed $\gamma$-species is observed for the lowest exposures. Its desorption maximum shifts from 280 to $265 \mathrm{~K}$ as the exposure increases. A pysisorbed $\beta$-species, with a desorption maximum shifting from 215 to $185 \mathrm{~K}$, evolves then, followed by condensation ( $\alpha$-species). Similar desorption spectra were measured by Vurens et al on an $\mathrm{Fe}$-oxide "multilayer film", which probably was identical to the $\mathrm{Fe}_{3} \mathrm{O}_{4}$ film prepared here [158]. $\mathrm{Fe}_{2} \mathrm{O}_{3}$ behaves very similar to $\mathrm{Fe}_{3} \mathrm{O}_{4}$. Only the desorption maxima of the chemisorbed $\gamma$-species appear at slightly lower temperatures shifting from 260 to $240 \mathrm{~K}$ with increasing exposure. The $\beta$ and and $\alpha$ signals are not clearly separated, the latter starts to desorb beyond $2.7 \mathrm{~L}$ with a common leading edge. 

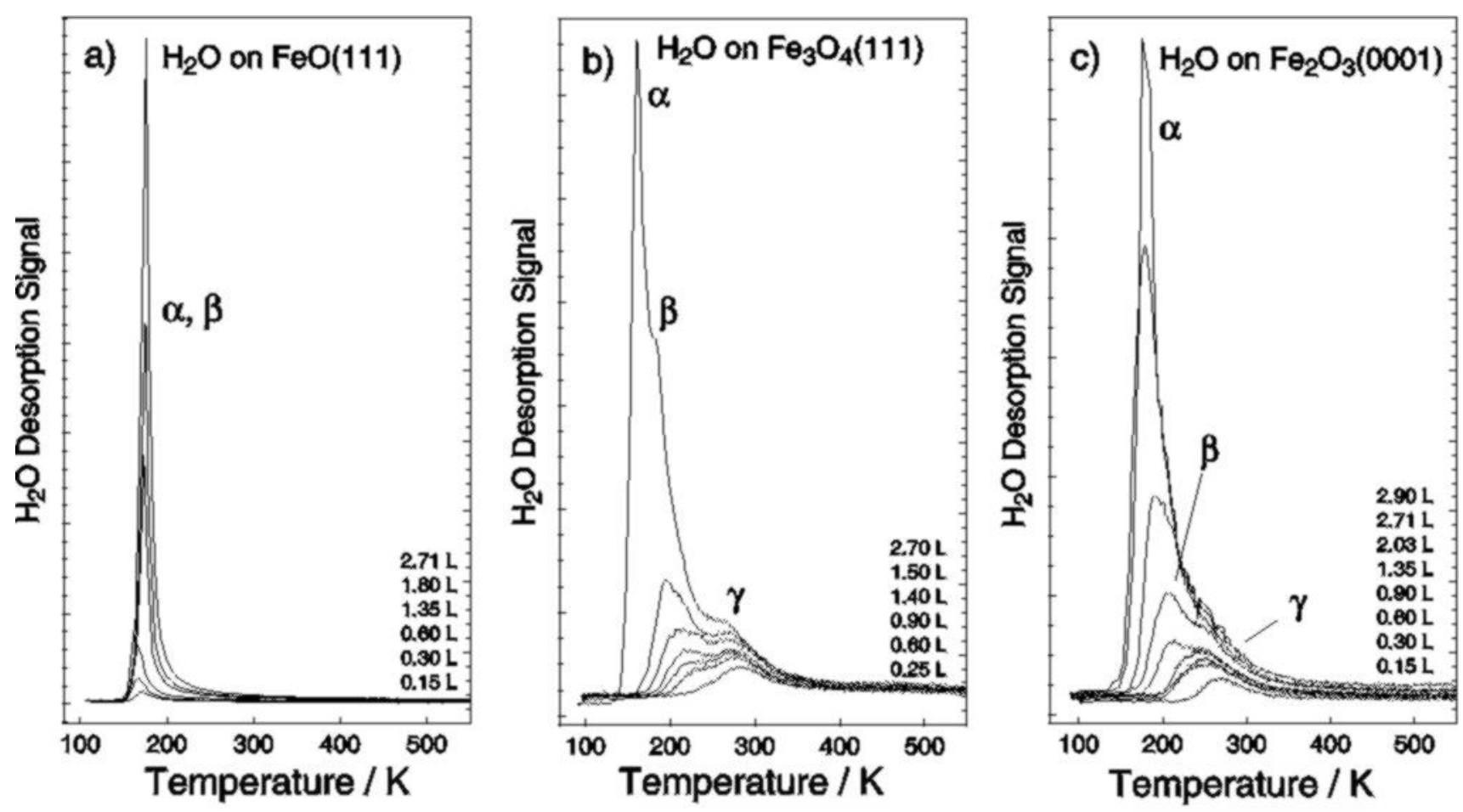

Fig. 6.16. Thermal desorption traces of water adsorbed on epitaxial Fe-oxide films grown onto $\mathrm{Pt}(111)$. (a) 1-2 ML thick $\mathrm{FeO}(111)$ film, (b) $\mathrm{Fe}_{3} \mathrm{O}_{4}$ (111) and (c) $\alpha-\mathrm{Fe}_{2} \mathrm{O}_{3}(0001)$ multilayer films. Exposures are given in units of Langmuir $\left(1 \mathrm{~L}=1.33 \times 10^{-6} \mathrm{mbar} \cdot \mathrm{s}\right)$. After $[88,366]$.

The shifts of the desorption signals of the chemisorbed and physisorbed species towards lower temperatures are attributed to repulsive adsobate-adsorbate interactions. The symmetrical shape of the chemisorbed $\gamma$-signal is consistent with second-order desorption kinetics, which also induces a shift of the desorption maximum with increasing coverage towards lower temperatures. These second-order kinetics are suggested by the observation of dissociatvely adsorbed water with UPS as presented in the next section. For physisorbed water first-order desorption kinetics are assumed.

An attempt was made to determine the desorption energies by a leading edge analysis. Since the uncertainty of the prefactors determined with this method is usually high, prefactors determined from a kinetic fit of the adsorption isobars measured by UPS (see Section 6.2.2) and from literature were used. The determined data are included in Table 6.4.

\subsubsection{UPS measurements - adsorbate identification and thermodynamics}

The valence-band spectra of adsorbed water were measured under A-D equilibrium conditions at constant water partial pressures and sample temperatures. As described for EB in Section 6.1.2, the relative adsorbate coverage was determined both from the adsorbate induced intensities $I_{a d}$ and from the attenuation of the substrate intensity measured $0.5 \mathrm{eV}$ below the Fermi level, expressed in terms of $d / l_{e}$ with $d$ the adsorbate layer thickness in units of the electron escape depth $l_{e}$. Again, both methods yield identical results [88]. Therefore, coverages will only be given in terms of $d / l_{e}$. In agreement with the TDS results, the adsorbate spectra reflect coverage dependent spectral shapes, due to sequential adsorption of chemisorbed, physisorbed and condensed water species. The species can best be distinguished by the analysis of incremental adsorbate spectra, i.e., the difference between adsorbate spectra with different coverage. 


\section{(i) Electronic structures of adsorbates}

Figure 6.17a shows the UP spectra of clean and water covered $\mathrm{FeO}(111)$ measured upon stepwise cooling of the sample, under a constant water pressure of $p\left(\mathrm{H}_{2} \mathrm{O}\right)=1 \times 10^{-8}$ mbar. They always show three features originating from the three highest occupied molecular orbitals of water. The feature around $-6 \mathrm{eV}$ corresponds to emission from the $\mathrm{O}$ lone-pair $1 \mathrm{~b}_{1}$ molecular orbital (MO), the feature between -7 and $-10 \mathrm{eV}$ is caused by the $3 \mathrm{a}_{1} \mathrm{MO}$ and that around $-13 \mathrm{eV}$ by the $1 \mathrm{~b}_{2} \mathrm{MO}$. The latter two MOs are involved in the $\mathrm{O}-\mathrm{H}$ bonds within the molecule. The $3 \mathrm{a}_{1} \mathrm{MO}$ is sensitive to the $\mathrm{H}-\mathrm{O}-\mathrm{H}$ bond angle. A detailed analysis of incremental spectra yields that the spectral shape changes at two distinct coverages representing saturation of the respective species. The corresponding saturation spectra are the two lower dotted curves, the upper dotted spectrum does not represent saturation, because it corresponds to condensed ice. This clearly indicates a sequential adsorption of three different species on $\mathrm{FeO}(111)$, whereas only two could be resolved in TDS.
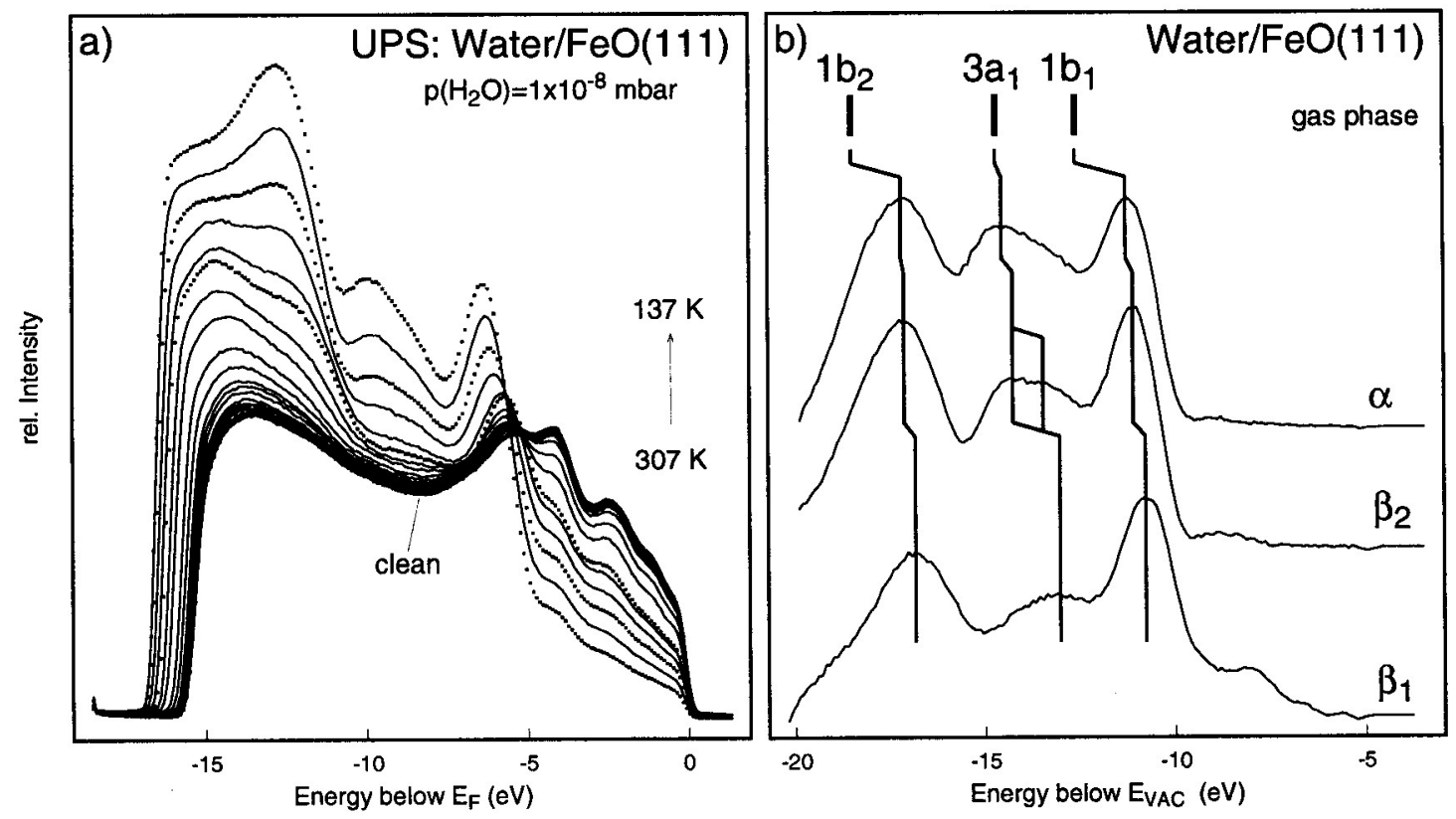

Fig. 6.17. (a) UP spectra of water on $\mathrm{FeO}(111)$ measured in adsorption-desorption equilibrium upon stepwise decreasing temperature. Spectra where $\beta_{1}$ and $\beta_{2}$ species are saturated and condensate $(\alpha)$ spectrum are dotted. (b) Incremental adsorbate spectra for observed water species on $\mathrm{FeO}(111): \beta_{1}$ (physisorbed monomeric water), $\beta_{2}$ (physisorbed $\mathrm{H}$ bonded water bilayer) and $\alpha$ (condensed ice). Bars indicate peak maxima positions of water gas-phase spectrum [320]. From [88].

Figure $6.17 \mathrm{~b}$ presents the incremental adsorbate spectra between the dotted spectra in Fig. 6.17a, labeled $\beta_{1}, \beta_{2}$ and $\alpha$. Compared to the peak positions of gas-phase water indicated by the bars [320] the whole spectrum of the initial adsorbate $\beta_{l}$ is shifted by a relaxation shift of $1.8 \mathrm{eV}$ towards lower BEs, but the relative energy separations are identical. This species is therefore interpreted as molecularly physisorbed monomeric water. It saturates at $d / l_{e}=0.55$. The work function decreases strongly. Assuming that saturation corresponds to a coverage, as in the model Fig. 6.21a, a dipole moment of 1.52 Debye units per molecule can be derived [88]. A species with similar properties observed on other surfaces has been described as two-dimensional lattice gas of water [337, 338]. The features between -5 and $-9 \mathrm{eV}$ below $E_{v a c}$ are ascribed to adsorbate induced changes in the substrate emission, which cannot be separated from the water induced emission. 
The $\beta_{2}$ species also represents physisorbed water and saturates around $d / l_{e}=1.3$, clearly more than twice the value for $\beta_{l}$. Its spectrum differs mainly by a broadening or split of the $3 \mathrm{a}_{1}$ peak. This is typical for a two-dimensional H-bonded phase, which leads to peak splittings, because of different BEs of proton-acceptor and proton-donor molecules, as calculated for a $\mathrm{H}$ bonded dimer [339, 340]. All acceptor orbitals are lowered with respect to the donor orbitals by the field of the positively charged bridging proton, leading to a splitting most clearly observable for the $3 \mathrm{a}_{1}$ orbital. The $3 \mathrm{a}_{1}$ peak in the spectrum of the condensed $\alpha$ species is shifted to an even higher BE and its split is less pronounced. This is typical for $\mathrm{H}$-bonded ice, where all water molecules have equivalent donor-acceptor-properties $[58,337]$. The average relaxation shifts evaluated from the positions of the $1 b_{1}$ and $1 b_{2}$ orbitals are also listed in Table 6.3.
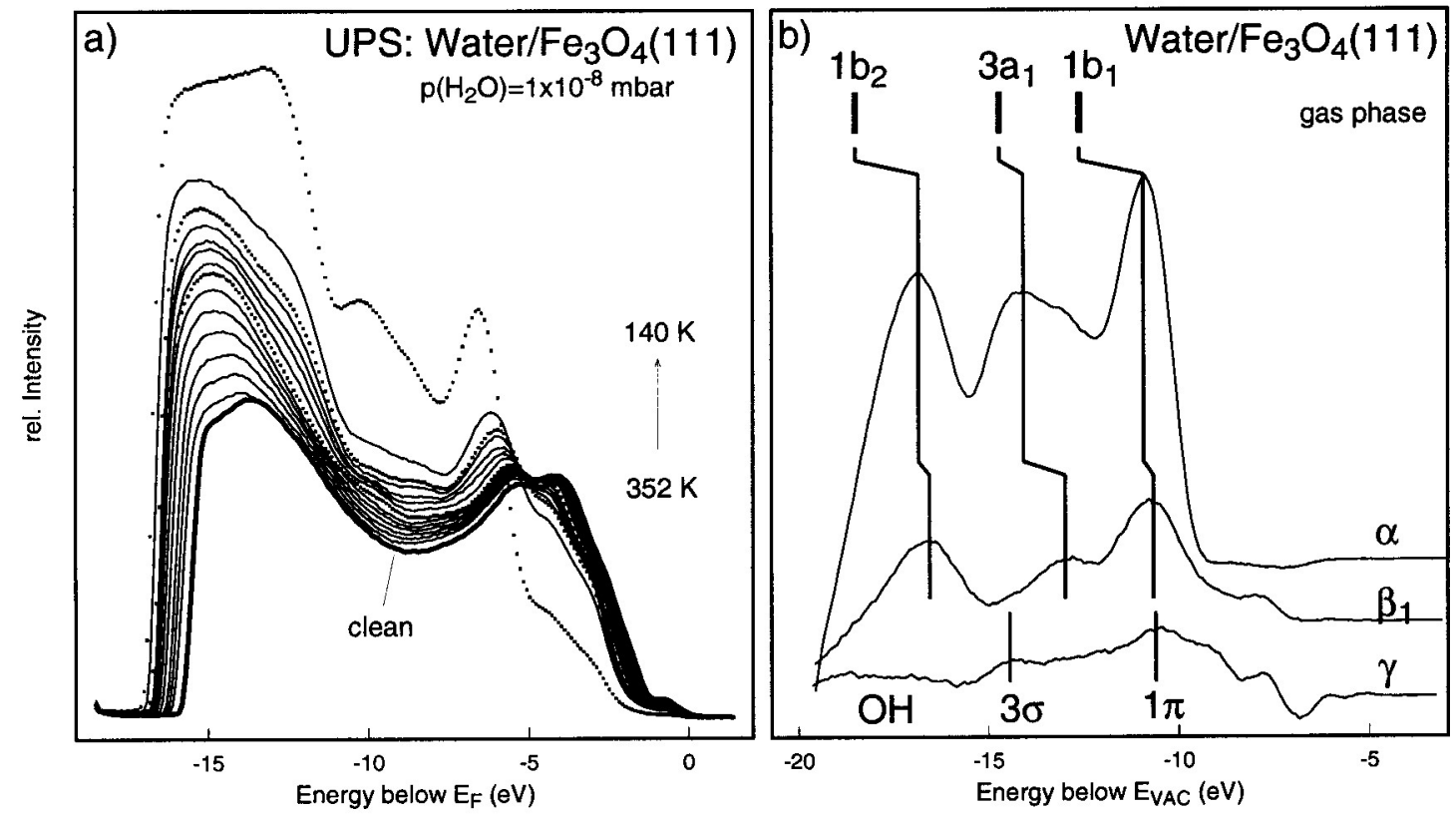

Fig. 6.18. (a) UP spectra of water on $\mathrm{Fe}_{3} \mathrm{O}_{4}(111)$ measured in A-D equilibrium upon stepwise decreasing temperature. Spectra where $\gamma$ and $\beta_{l}$ species are saturated and condensate $(\alpha)$ spectrum are dotted. (b) Incremental adsorbate spectra: $\gamma$ (dissociated water resulting in $\mathrm{OH}+\mathrm{H}$ ) $\beta_{l}$ (physisorbed monomeric water) and $\alpha$ (condensed ice). Bars indicate peak maxima positions of water gas-phase spectrum [320]. From [88].

Figure 6.18a shows the corresponding UP spectra of $\mathrm{Fe}_{3} \mathrm{O}_{4}(111)$ measured at $\mathrm{p}\left(\mathrm{H}_{2} \mathrm{O}\right)=1 \times 10^{-8}$ mbar during stepwise cooling. The incremental adsorbate spectra upon saturation of the different sequentially adsorbing species are shown in Fig. 6.18b. Three species can be distinguished. The first one $(\gamma)$ has only two main broad peaks and saturates at $d / l_{e}=0.43$. It is attributed to adsorbed $\mathrm{OH}$ species resulting from dissociation of water to form $\mathrm{OH}+\mathrm{H}$. The two peaks are due to the $1 \pi$ and $3 \sigma$ orbitals of $\mathrm{OH}$ as interpreted similarly for $\mathrm{OH}$ in $\mathrm{NaOH}$ [341] and on other metal-oxides [58, 342-344]. We also attribute the features between -5 and $-10 \mathrm{eV}$ to adsorbate induced changes in the substrate emission and possibly to emission from the coadsorbed $\mathrm{H}$ atom. From the work function decrease and a saturation coverage as in Fig. 6.21c, a dipole moment of 0.92 Debye units per $\mathrm{OH}+\mathrm{H}-$ unit is derived [88]. The spectrum of the second species is identical to that of the $\beta_{1}$ species on FeO concerning both shape and peak positions. It, thus, corresponds to physisorbed monomeric undissociated water. It saturates at a total coverage of $d / l_{e}=0.86 \pm 0.03$, exactly twice the value of the $\gamma$ species. Finally, the spectrum of condensed ice $(\alpha)$ is observed. 


\section{(ii) Isosteric heats of adsorption}

Figure 6.19a compares the adsorption isobars for $\mathrm{FeO}(111)$ and $\mathrm{Fe}_{3} \mathrm{O}_{4}(111)$ determined from UPS measurements at three water partial pressures $p\left(\mathrm{H}_{2} \mathrm{O}\right)$. The saturation coverages as determined from the difference spectra generally agree with breaks in the isobars indicated by the horizontal lines, especially for $\gamma+\beta_{1}$ on $\mathrm{Fe}_{3} \mathrm{O}_{4}$ and for $\beta_{1}$ on $\mathrm{FeO}$. For each oxide, the isobars look very similar in shape and are almost equidistant at all coverages.
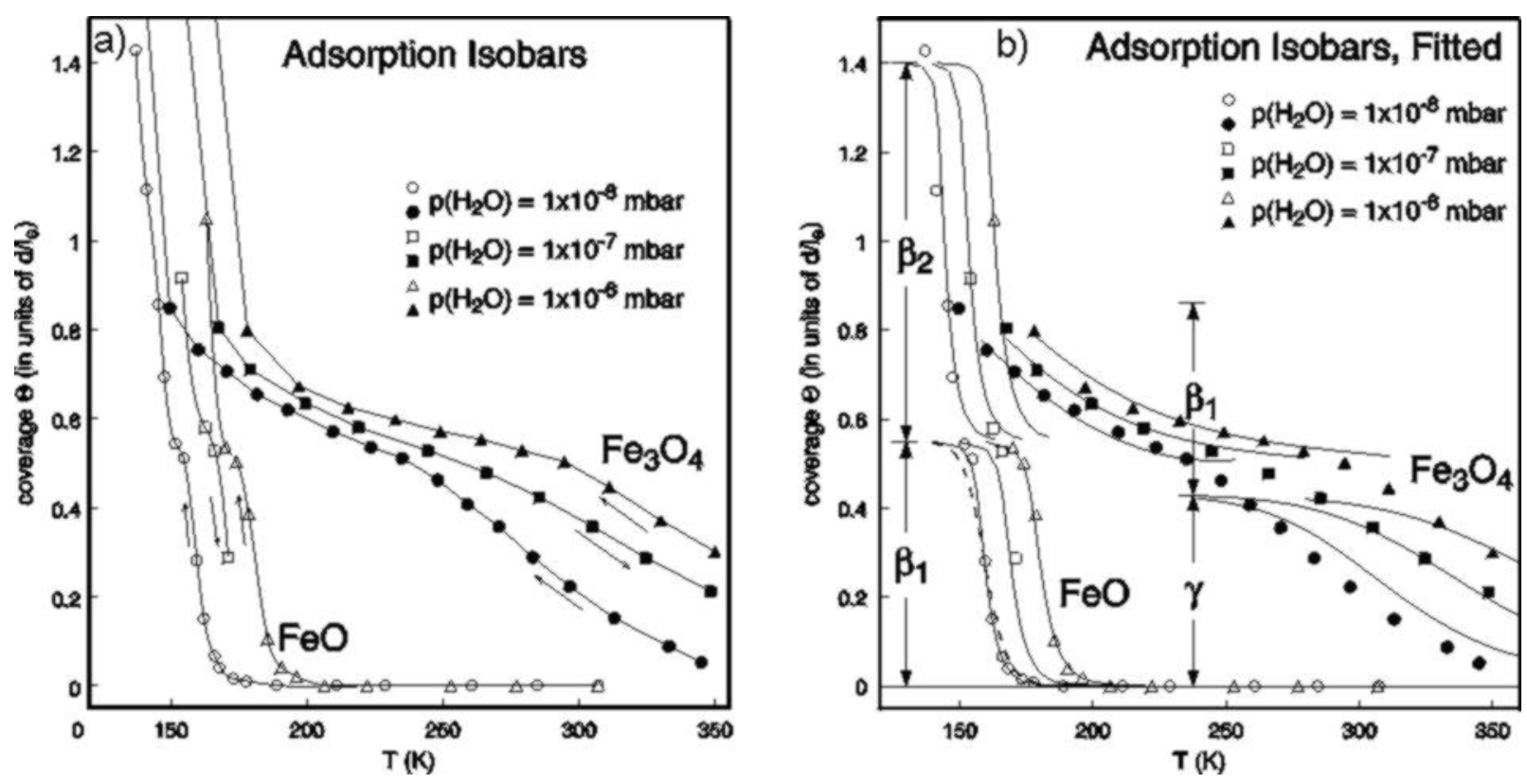

Fig. 6.19. (a) Adsorption isobars of water at indicated partial pressures on $\mathrm{FeO}(111)$ and $\mathrm{Fe}_{3} \mathrm{O}_{4}(111)$. Adsorption range and saturation coverages of different adsorbate species are marked. Arrows indicate "direction" of measurements by decreasing or increasing temperature (b) Same data with fitted curves, see text. From [88].

As described in Section 2.4 and applied for EB in Section 6.1.2, isosteric heats of adsorption $q_{s t}(\Theta)$ were determined. Together with the desorption energies $E_{d e s}$ determined by TDS they are plotted in Fig.6.20 as a funtion of the coverage $\Theta$ in terms of $d / l_{e}$ and are listed in Table 6.3.

The experimental adsorption isobars should follow one of the isobar equations (2.11), (2.12) (firstand second-order Langmuir), (2.18) (first-order with mobile precursor) or (2.19) (quasi-first-order, mobile precursor) derived in Sections 2.3 and 2.5. Figure $6.19 \mathrm{~b}$ shows the resulting fitted curves obtained by adjusting the kinetic parameters $n$ (desorption order), $v_{n}$ (frequency factor for desorption) and $K$ (Kisliuk factor). The isosteric heats of adsorption $q_{s t}(\Theta)$ from the Clausius-Clapeyron analysis are independent of the kinetics and need not be fitted. The saturation densities $\sigma_{A}$ are assumed according to the models discussed in Sections 5.3 and 5.5. In case of dissociative water adsorption on $\mathrm{Fe}_{3} \mathrm{O}_{4}(111)$, it is equal to the density of surface Fe sites, i.e., $\sigma_{A}=3.3 \times 10^{14} \mathrm{~cm}^{-2}$. For the other adsorbate species, the values are similar. The initial sticking coefficient $s^{*}$ was always assumed to be 1 . Since the equations for $b_{1}$ (2.11) and $b_{2}$ (2.12) contain only the ratios $s^{*} / v_{n}$, smaller values of $s^{*<1}$ would result in correspondingly smaller frequency factors $v_{n}$. The deduced values for $q_{s t}$ and $v_{n}$ are included in Tables 6.3 and 6.4 . 

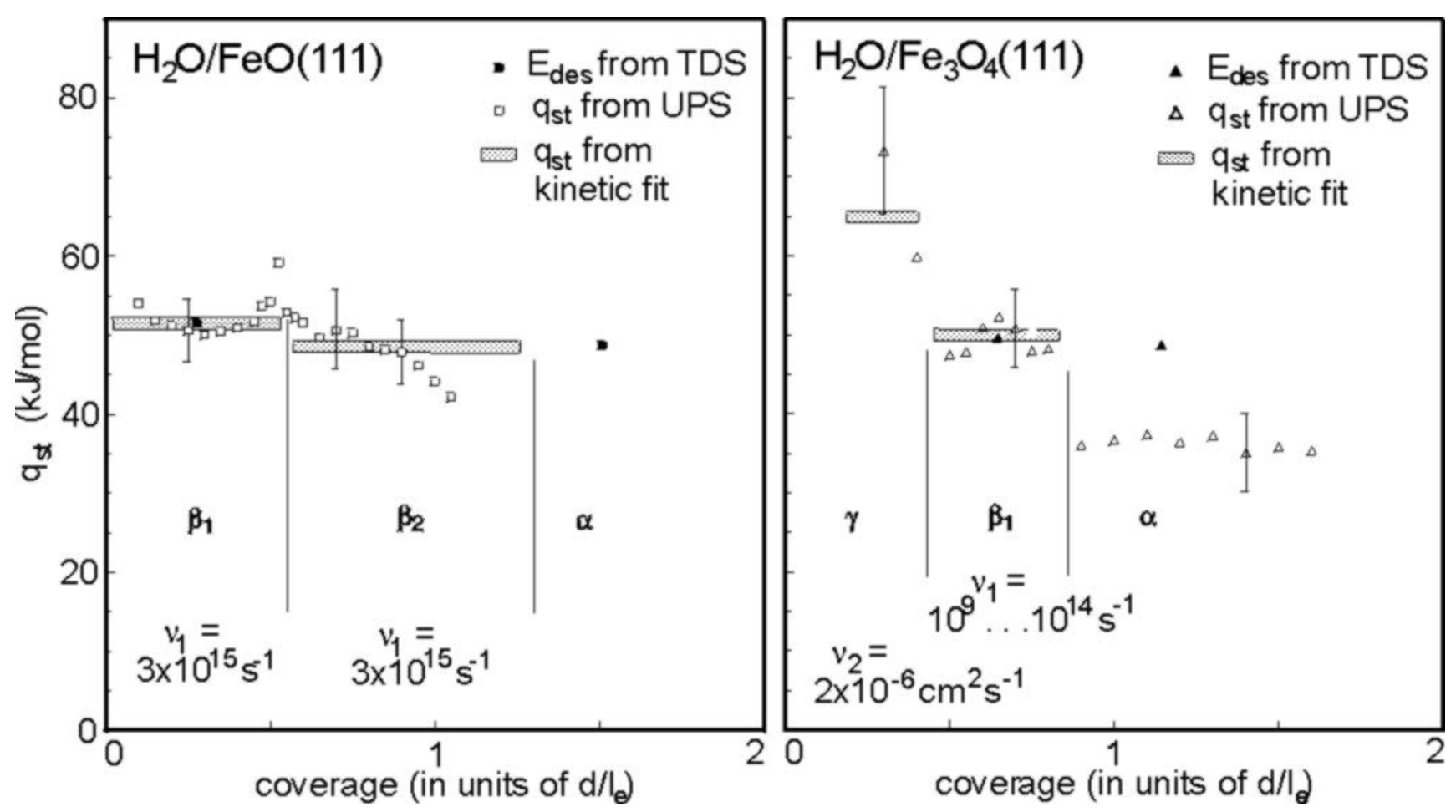

Fig. 6.20. Isosteric heats of adsorption $q_{s t}(\Theta)$ and desorption energies $E_{d e s}$ versus coverage in terms of $d / l_{e}$ for water on $\mathrm{FeO}(111)$ (a) and $\mathrm{Fe}_{3} \mathrm{O}_{4}(111)(\mathrm{b})$. Coverage ranges of different adsorbate species are indicated. After [88].

The fit of the isobars in the range of molecular $\beta_{l}$-adsorption on $\mathrm{FeO}$ was tried with the usual firstorder Langmuir adsorption and desorption kinetics using (2.11) (dashed curve in Fig. 6.19b). However, the steep coverage decrease with $T$ is much better simulated when including a mobile adsorption precursor kinetics, as described by (2.18) (solid lines in Fig. 6.19b). The resulting fit values are $q_{s t}=52$ $\mathrm{kJ} / \mathrm{mol}$, which agrees perfectly with the Clausius-Clapeyron (C-C) evaluation (Fig. 6.20), and $\mathrm{v}_{l}=$ $3 \times 10^{15} \mathrm{~s}^{-1}$. This frequency factor is comparatively high. According to the transition state theory this means that the activated complex has a high degree of freedom when compared to the adsorbed state [16]. This transition state might be indendical with the mobile precursor state in the adsorption process. A Readhead analysis of the TDS data, assuming a frequency of $10^{13} \mathrm{~s}^{-1}$, yields a desorption energy $E_{\text {des }}=41 \mathrm{~kJ} / \mathrm{mol}$, which is definitely too small. Using the value $\mathrm{v}_{l}=3 \times 10^{15} \mathrm{~s}^{-1}$, as determined from the isobar analysis, however, yields $E_{d e s}=52 \mathrm{~kJ} / \mathrm{mol}$ in perfect agreement with the value from the C-C analysis.

The fit in the $\beta_{2}$ adsorption range is less reliable, because this range is quite narrow, so that only comparatively few data points were measured and because the transition to condensation occurs without visible shoulder in the isobars. A saturation of the $\beta_{2}$ species at $d / l_{e}=1.4$, as expected from the models discussed below, has been used. A fit is only possible when a first-order mobile precursor kinetics are assumed. The fitted values are $q_{s t}=47 \pm 2 \mathrm{~kJ} / \mathrm{mol}$, which also agrees well with the average value from the $\mathrm{C}-\mathrm{C}$ evaluation, and $\mathrm{v}_{1}=3 \times 10^{(15 \pm 1)} \mathrm{S}^{-1}$. Since no $\beta_{2}$ desorption peak was resolved in TDS, comparison is not possible.

The shape of the isobars for the $\gamma$-species on $\mathrm{Fe}_{3} \mathrm{O}_{4}$ can be fitted only with (2.12) assuming dissociative adsorption and second-order desorption kinetics, which agrees with the UPS observation that the $\gamma$-species corresponds to dissociated water. Assuming quasi first-order desorption (2.19) yields steeper isobars incompatible with the experimental results, which means that the $\mathrm{OH}$ and $\mathrm{H}$ species are 
spatially separated and adsorbed independently. The fit was adjusted to the isobar for $10^{-7} \mathrm{mbar}$ and fits the isobar for $10^{-6}$ mbar with the same parameters. The calculated isobar for $10^{-8} \mathrm{mbar}$ has the same shape as the experimental curve. However, the experimental data reflect too low coverages. Probably the sticking coefficient is low, so that equilibrium coverage was not established within the waiting period at this low pressure. This low sticking coefficient may partly be due to its coverage dependence $s=s_{0}(1-\Theta)^{2}$ for dissociative adsorption, but also an activation barrier for dissociation cannot be ruled out.

The $\gamma$-isobar fit on $\mathrm{Fe}_{3} \mathrm{O}_{4}$ yields $q_{s t}=65 \pm 2 \mathrm{~kJ} / \mathrm{mol}$ and $v_{2}=(2.4 \pm 1) \times 10^{-6} \mathrm{~cm}^{2} \mathrm{~s}^{-1}$. Whereas the frequency factor derived from TDS assuming second-order desorption kinetics $\left(\mathrm{v}_{2}=10^{-(5 \pm 2)} \mathrm{cm}^{2} \mathrm{~s}^{-1}\right)$ is of the same order of magnitude, the desorption energy from TDS $\left(E_{d e s}=50 \pm 10 \mathrm{~kJ} / \mathrm{mol}\right)$ is too low. This is due to the overlap of the desorption peaks for the $\gamma$ and $\beta$ species in Fig. 6.16b, which makes $T_{\max }$ and its coverage dependence difficult to extract. The low second-order frequency factor $v_{2}$ makes the desorption process slow and suggests that $\mathrm{OH}$ and $\mathrm{H}$ have to assume a sterically demanding configuration in order to recombine and to desorb. This low frequency factor also explains why considerable amounts of the $\gamma$ species are adsorbed under equilibrium pressure at room temperature, although the heat of adsorption is not much higher than for the physisorbed $\beta$ species. Adsorption into the $\gamma$ state is fast, but desorption is slow.

The experimental isobars for the molecular $\beta_{1}$-species coadsorbed after saturation of the $\gamma$-species on $\mathrm{Fe}_{3} \mathrm{O}_{4}$ extend over a very wide temperature range and cannot be fitted with both $q_{s t}$ and $v_{1}$ being coverage-independent. Assuming $v_{l}$ to be constant, results in $q_{s t}$ values, which for small $\beta_{l}$ coverages are in the range of the $\gamma$-species $(65 \mathrm{~kJ} / \mathrm{mol})$. With increasing coverage they decrease to a final value similar to that for the $\beta_{1}$-species on $\mathrm{FeO}(52 \mathrm{~kJ} / \mathrm{mol})$. The $\mathrm{C}$-C evaluation gave no indication for such a coverage dependence, but suggests a fairly constant value of $q_{s t}=48-52 \mathrm{~kJ} / \mathrm{mol}$. Therefore, a constant value of $q_{s t}=50 \mathrm{~kJ} / \mathrm{mol}$ was assumed and the frequency factor for molecular first-order desorption $v_{l}$ was adjusted using (2.12). The resulting value for $v_{l}$ descreases from $10^{9}$ for $\Theta_{\beta l}=0.1$ over $10^{12}$ for $\Theta_{\beta 1}=0.5$ to $10^{14}$ for $\Theta_{\beta I}=0.8$, where the last value approaches that of the $\beta_{l}$-species on $\mathrm{FeO}$. The very low initial values of $\mathrm{v}_{l}$ again suggest an activated complex with reduced degrees of freedom, when compared to the adsorbed state. Since this coverage dependence does not exist for the $\beta_{l}$-species on $\mathrm{FeO}$, it was proposed that it is due to the formation of $\gamma+\beta_{1}$ coadsorption complexes, where the molecules are confined into more restricted configurations, resulting in reduced translational and vibrational degrees of freedom and, thus, lower frequency factors [88]. Such complexes, consisting of coadsorbed molecular and dissociated water species, were predicted recently by ab-initio calculations for $\mathrm{MgO}(100)$ [345] and $\mathrm{TiO}_{2}(110)$ surfaces [346].

\section{(iii) Adsorption models}

Figure 6.21 displays models for water adsorption on $\mathrm{FeO}(111)(\mathrm{a}, \mathrm{b})$ and $\mathrm{Fe}_{3} \mathrm{O}_{4}(111)(\mathrm{c}, \mathrm{d})$., which are in agreement with the following findings, obtained from the films with the lowest surface defect concentration: (i) the coverages of the $\gamma$ and the $\beta_{l}$ species on $\mathrm{Fe}_{3} \mathrm{O}_{4}(111)\left(\mathrm{d} / \mathrm{l}_{\mathrm{e}}=0.43\right.$ each)correspond to the surface Fe atom density $\left(3.3 \times 10^{14} \mathrm{~cm}^{-2}\right)$, (ii) the coverage of $\beta_{2}$ on $\mathrm{FeO}$ in terms of $d / l_{e}$ is more than twice that of $\beta_{l}$, (iii) the $\gamma$-species is dissociated $\mathrm{OH}+\mathrm{H}$, all $\beta$-species are molecular water; the $\beta_{1^{-}}$ species on $\mathrm{FeO}$ and $\mathrm{Fe}_{3} \mathrm{O}_{4}$ are not, but the $\beta_{2}$-species on $\mathrm{Fe}_{3} \mathrm{O}_{4}$ is $\mathrm{H}$-bonded, (iv) the large work function changes require an adsorption geometry with the $\mathrm{O}$ atom downwards in all cases, and (v) the relative coverages of the different species in terms of $d / l_{e}$ must be reproduced exactly. 


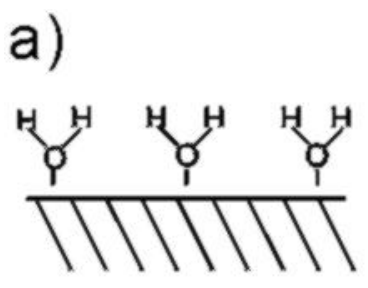

$\mathrm{FeO}(111), \beta_{1}$

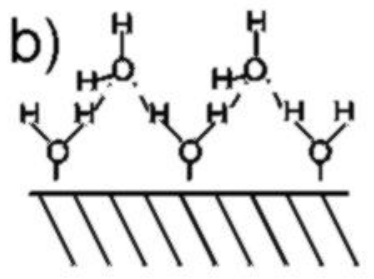

$\mathrm{FeO}(111), \beta_{2}$

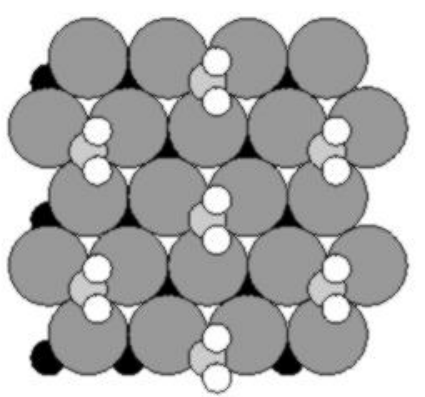

C)

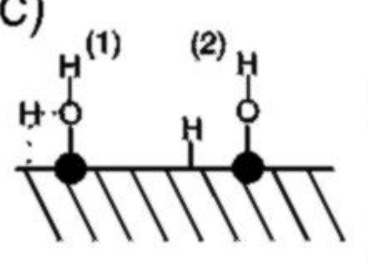

$\mathrm{Fe}_{3} \mathrm{O}_{4}(111), \gamma$
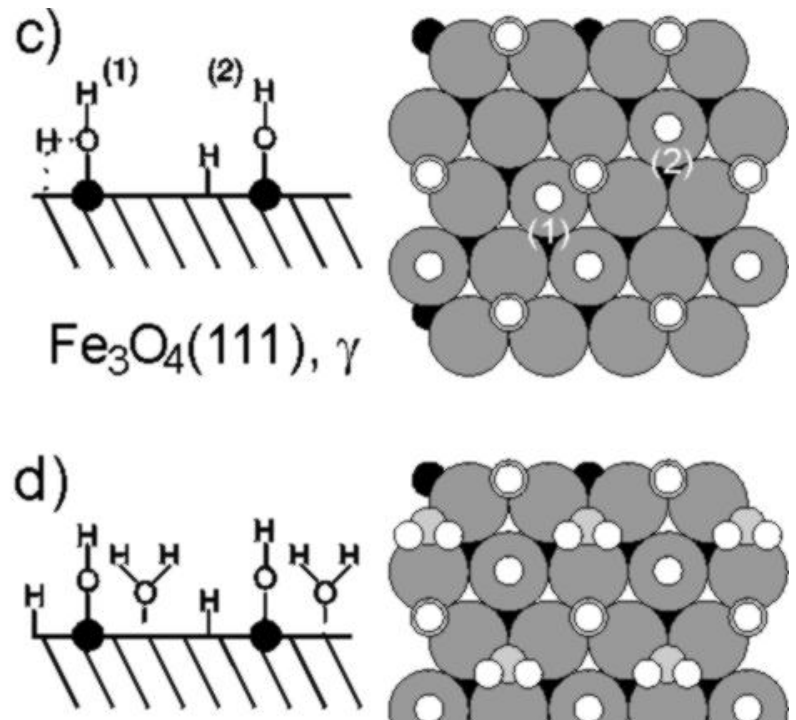

$\mathrm{Fe}_{3} \mathrm{O}_{4}(111), \gamma+\beta_{1}$

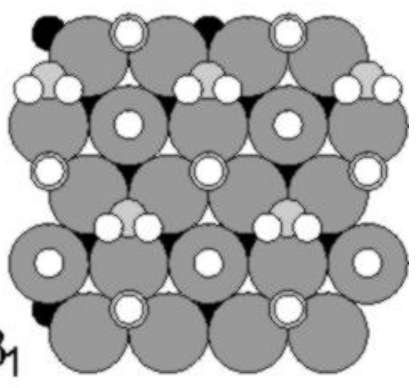

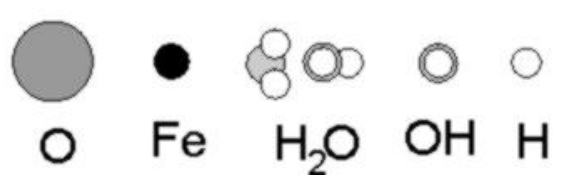

Fig. 6.21. Adsorption models for water on $\mathrm{FeO}(111)$ and $\mathrm{Fe}_{3} \mathrm{O}_{4}(111)$. (a) Monomeric $\beta_{1}$ water physisorbs onto $\mathrm{O}$ hollow sites of $\mathrm{FeO}(111)$. (b) Complete water bilayer on $\mathrm{FeO}(111)$ consisting of monomeric $\beta_{1}$ water in first layer and $\mathrm{H}$ bonded physisorbed water $\beta_{2}$ above. (c) Dissociatively chemisorbed $\gamma$ water on $\mathrm{Fe}_{3} \mathrm{O}_{4}(111)$ with $\mathrm{OH}$ groups adsorbed on surface Fe cations (which therefore are invisible in top view) and $\mathrm{H}$ atoms adsorbed on $\mathrm{O}$ anions. (d) $\mathrm{Fe}_{3} \mathrm{O}_{4}(111)$ surface completely covered by $\mathrm{OH}$ and $\mathrm{H}$ groups $(\gamma)$ and monomerically physisorbed $\beta_{l}$ water coadsorbed onto surface $\mathrm{O}$ hollow sites. After [88].

On $\mathrm{FeO}(111)$, monomeric $\beta_{1}$-water physisorbs in the first layer up to saturation at $d / l_{e}=0.55$ (Fig. 6.21a). It is bound to the surface via the $\mathrm{O}$ lone pair-orbital with the $\mathrm{H}$ atoms oriented upwards as deduced from the high initial dipole moment of $1.52 \mathrm{D}$, which is only slightly smaller than for the free water molecule $(1.84 \mathrm{D})$. This adsorbate layer corresponds to a $(\sqrt{3} \times \sqrt{3}) \mathrm{R} 30^{\circ}$ superstructure with respect to $\mathrm{FeO}(111)$ with an intermolecular distance of $5.37 \AA$. However, the corresponding superstructure spots were not observed in the LEED pattern, which suggests a high mobility of the adsorbed species. Therefore, the model in Fig. 6.21a represents an idealized frozen structure. The spectra of the second physisorbed species $\left(\beta_{2}\right)$ indicate $H$ bonding, and their saturation coverage is more than twice that of the $\beta_{1}$ species. This points towards the formation of a water bilayer, Fig. $6.21 \mathrm{~b}$, as that observed on several metal surfaces [58] and first on $\operatorname{Pt}(111)$ [347]. The second water layer connects the water molecules of the first layer via $\mathrm{H}$ bonds with one $\mathrm{H}$ atom pointing away from the surface, resulting in buckled hexagonal rings, as in ice structures. The in-plane distance between the water molecules in ice is $4.53 \AA$, which corresponds to the distance between the lower molecules in the buckled rings in Fig. $6.21 \mathrm{~b}$. Since this distance is $0.5 \AA$ shorter than the intermolecular distance in the first $\beta_{l}$ layer, the $\beta_{l}$ layer must be compressed. Further water adsorption results in the formation of thick ice layers. LEED shows that no long-range order is established. 
We assume that the exposed $\mathrm{Fe}$ cations on the $\mathrm{Fe}_{3} \mathrm{O}_{4}(111)$ surface act as acidic sites that bind the $\mathrm{OH}$ groups formed after dissociation of water. At first, the protons are adsorbed on neighboring $\mathrm{O}$ atoms (step 1 in Fig. 6.21c), then they may move to the $\mathrm{O}$ atoms not coordinated to surface Fe atoms (step 2 and right hand atomic model in Fig. 6.21c). This could also be regarded to result in $\mathrm{OH}$ groups, but as these $\mathrm{O}$ atoms belong to the substrate lattice, the interaction with the $\mathrm{H}$ atom is supposed to be weaker than in the $\mathrm{OH}$ groups adsorbed on the Fe cations. The back reaction needs the reestablishment of step 1, as intermediate, which may represent the transition state for desorption. This spatially and sterically demanding arrangement may be responsible for the observed low second-order frequency factor for desorption. The $\mathrm{OH}$ group and the adsorbed $\mathrm{H}$ atom represent the $\gamma$-species shown in Fig. $6.21 \mathrm{c}$, which saturates at $d / l_{e}=0.43$. As the coverage increases, further monomeric $\beta_{l}$ water is coadsorbed onto $\mathrm{O}$ hollow sites between the adsorbed $\mathrm{OH}$ and $\mathrm{H}$ groups (Fig. 6.21d) until saturation at $d / l_{e}=0.86$ is reached. This unusual behavior is clearly indicated by the UP spectrum for monomeric water observed in Fig. 6.18.b, which is identical to the monomeric water spectrum on $\mathrm{FeO}(111)$ in Fig. $6.17 \mathrm{~b}$. The extremely low frequency factor for desorption in the low-coverage regime of of this $\beta_{l}$ species suggests a stabilizing interaction with the $\mathrm{OH}$ and $\mathrm{H}$ groups. A second physisorbed $\beta_{2}$ layer as on $\mathrm{FeO}(111)$ cannot be formed, because the distance between the $\beta_{1}$ water molecules locked beween the adsorbed $\mathrm{OH}$ and $\mathrm{H}$ species is too large for bilayer formation. Therefore, further water adsorption results in a thick ice layer.

\section{(iv) The role of surface defects}

From the full-width of half maximum (FWHM) of the (00) beam, as a function of the electron primary energy, as well as from the background intensities in the LEED patterns, the concentration of atomic steps and point defects were quantified on different $\mathrm{Fe}_{3} \mathrm{O}_{4}(111)$ surfaces [88]. The UPS data presented above were for the best-ordered film. The UP spectra for water adsorbed on films with different surface defect concentrations all are very similar in general shape. They all show sequential adsorption of $\mathrm{OH}+\mathrm{H}(\gamma)$, physisorbed $\beta_{l}$ and condensed $\alpha$ water. The main difference is that the $\gamma$ species saturate at lower coverages on the films with higher surface point-defect concentration, indicating less water to dissociate and form $\mathrm{OH}$ and $\mathrm{H}$ species. According to the interpretation presented in the previous section, this means that the concentration of Fe cations exposed in the topmost layer is lower. This interpretation is in line with the identification of the dominating type of point defects on $\mathrm{Fe}_{3} \mathrm{O}_{4}(111)$ as missing Fe cations, as discussed in Section 5.5. It substantiates the dissociative adsorption of water to take place on regular and defect-free surface areas of $\mathrm{Fe}_{3} \mathrm{O}_{4}(111)$. However, water dissociation also might also occur on other types of surface defects, such as atomic steps.

\subsubsection{Discussion}

Water adsorption studies on metal-oxide surfaces were initiated by work on its photocatalytic decomposition on $\mathrm{TiO}_{2}$ [348], followed by investigations on other oxides and ionic compounds [5, $338,349,350]$. Molecular adsorption mostly occurs via the lone-pair of the $\mathrm{O}$ atom to an acidic surface site [58]. If water dissociates, resulting in $\mathrm{OH}$ and $\mathrm{H}$, a Lewis acidic adsorption site is needed to adsorb the $\mathrm{OH}$ species and a Brønsted basic site should be in the neighborhood to accept the $\mathrm{H}$ atom. For this reason, water dissociation on metals is often observed only when $\mathrm{O}$ is preadsorbed [58], as was observed first on $\mathrm{Pt}(111)$ [351]. On non-polar oxide surfaces, such as $\mathrm{CoO}(100)$ [342] and $\mathrm{NiO}(100)$ $[64,344]$ dissociative adsorption is found to occur only at defects, whereas the corresponding regular surface areas are inert. Dissociative adsorption was observed on $\mathrm{Ti}^{3+}$ rich $\mathrm{Ar}^{+}$-bombarded surfaces, such as $\mathrm{Ti}_{2} \mathrm{O}_{3}(047)$ [352], $\mathrm{TiO}_{2}(100)$ [353], $\mathrm{SrTiO}_{3}(100)$ [354], whereas molecular adsorption was observed on the respective stoichiometric regular surfaces. On $\mathrm{MgO}(100)$ [343] and $\alpha-\mathrm{Fe}_{2} \mathrm{O}_{3}(0001)$ 
$[136,349]$, water was found to dissociate both on regular and defective surfaces and a correlation between the defect concentration and amount of dissociative adsorption was found. On other oxides, the role of defects for the dissociative adsorption is not clear, because water dissociation occurs both on ordered and defective surfaces with a similar rates, as on $\mathrm{V}_{2} \mathrm{O}_{3}(047)$ [135], $\mathrm{TiO}_{2}(110)$ [355], $\mathrm{TiO}_{2}$ (001) [356] and $\mathrm{SnO}_{2}(110)$ [350]. These studies suggest that defect sites can mostly dissociate water, in agreement with many studies performed on polycrystalline oxide materials [3]. The reactivity of defect-free, regular oxide surfaces towards water dissociation depends most likely on the specific surface structure. The non-polar surfaces are usually less reactive, because the metal cations are shielded by the large $\mathrm{O}$ anions.

On the epitaxial $\mathrm{FeO}(111)$ and $\mathrm{Fe}_{3} \mathrm{O}_{4}(111)$ films, all water species $\left(\mathrm{H}_{2} \mathrm{O}\right.$ and $\left.\mathrm{OH}\right)$ adsorb with the $\mathrm{O}$ atoms oriented towards the substrate surfaces, as indicated by the large decrease of the work function. Analogous to the adsorption of ethylbenzene, water physisorbs molecularly and without significant chemical interaction onto the O-terminated $\mathrm{FeO}(111)$ surface, followed by the formation of a physisorbed bilayer and finally by condensation of ice. The initial physisorption of water monomers represents an unusual behavior that differs from the common adsorption behavior on metals, where water forms mostly bilayer islands or cluster-like aggregates from the beginning. Electronically, the $\mathrm{FeO}(111)$ surface seems to be "corrugated" for the water molecules by the Fe cations in the second layer (see Fig. 5.3b). As discussed in Section 5.3, the $\mathrm{FeO}(111)$ film is strongly relaxed with less ionic surface $\mathrm{O}$ atoms. The lattice is laterally expanded, so that the $\mathrm{O}$ atoms of the water molecules may interact with the Fe atoms below the topmost $\mathrm{O}$ layer, when adsorbed in $\mathrm{O}$ hollow sites. This explains the unusual adsorption geometry with the water $\mathrm{O}$ atom oriented towards the $\mathrm{O}$-terminated $\mathrm{FeO}(111)$ surface. Since the Fe-Fe nearest neighbour distance is too small for the water molecules, they assume the next-nearest packing, which corresponds to $(\sqrt{3} \times \sqrt{3}) \mathrm{R} 30^{\circ}$, as in Fig. 6.21a. Here, their distance is already too large for the formation of $\mathrm{H}$-bonded aggregates.

On the Fe-terminated $\mathrm{Fe}_{3} \mathrm{O}_{4}(111)$ surface, water chemisorbs dissociatively, followed by physisorption of monomers and final condensation of ice. From the saturation coverages it follows that one $\mathrm{OH}+\mathrm{H}$ species and one physisorbed $\mathrm{H}_{2} \mathrm{O}$ monomer per $\mathrm{Fe}_{3} \mathrm{O}_{4}(111)$ surface unit cell adsorb sequentially, before condensation of ice starts. The dissociation occurs by the simultaneous action of acidic surface Fe cations and neighboring basic $\mathrm{O}$ anions. The resulting $\mathrm{OH}$ groups are bound by the Fe cations and the $\mathrm{H}$ atoms by the $\mathrm{O}$ anions. After saturation of the $\mathrm{OH}$ and $\mathrm{H}$ species monomeric water molecules are coadsorbed onto $\mathrm{O}$ hollow sites, located between the $\mathrm{OH}$ and $\mathrm{H}$ species. This unexpected behavior is clearly indicated by the UP spectra of monomeric water, which rules out molecular adsorption of water onto the already adsorbed $\mathrm{OH}$ and $\mathrm{H}$ groups via $\mathrm{H}$ bonds. The physisorption of water molecules onto $\mathrm{O}$ hollow sites with the $\mathrm{O}$ atom oriented downwards probably is favored by the strongly reduced interlayer distance between the topmost $\mathrm{O}$ layer and the Fe layer underneath, as determined by the LEED analysis in Section 5.5. This could support an interaction between the water $\mathrm{O}$ atom and Fe cations in the second layer. The arrangement in Fig. 6.21d shows the water molecules on hollow sites without Fe cations below. This is based on the assumption of repulsive interaction between $\mathrm{H}_{2} \mathrm{O}, \mathrm{OH}$ and $\mathrm{H}$. In fact, indications for the formation of adsorption complexes were discussed in the previous section. Since no indications of $\mathrm{H}$ bonding were observed, the actual arrangement remains speculative.

The dissociative adsorption of water occurs on regular defect-free $\mathrm{Fe}_{3} \mathrm{O}_{4}(111)$ surface areas. The introduction of the dominant type of point defect, missing Fe atoms in the topmost layer, reduces the saturation coverage of $\mathrm{OH}+\mathrm{H}$. This substantiates the role of surface metal cations as reactive sites on $\mathrm{Fe}_{3} \mathrm{O}_{4}(111)$ and on metal-oxide surfaces in general. On $\alpha-\mathrm{Fe}_{2} \mathrm{O}_{3}(0001)$ TDS measurements have shown a very similar sequence of chemisorption, physisorption and condensation of water. The chemisorbed species is more weakly bound than on $\mathrm{Fe}_{3} \mathrm{O}_{4}(111)$ and, since UPS measurements have not been performed so far, it is not known if it also is dissociated. Most likely, this surface is O terminated and it 
is possible that the adsorbate feels the acidic character of the second layer Fe atoms, as was discussed for initial adsorption of EB and St (Sections 6.1 and 6.3).

TABLE 6.3. Saturation coverages $\Theta_{s a t}$, isosteric heats of adsorption $q_{s t}$, frequency factors $v_{n}$ and desorption order $n$ of different water species adsorbed on epitaxial Fe-oxide films obtained from kinetic fit of adsorption isobars measured by UPS. Also listed are relaxation shifts $\Delta E_{R}$, MO peak positions in UP spectra and desorption energies $E_{\text {des }}$ from TDS evaluations.

\begin{tabular}{|c|c|c|c|c|c|c|c|c|}
\hline Substrate & $\begin{array}{l}\text { Adsorbate } \\
\text { species }\end{array}$ & $\begin{array}{c}\Theta_{\text {sat }} \text { in units } \\
\text { of } d / l_{e}\end{array}$ & $\begin{array}{l}\Delta E_{R} \\
(\mathrm{eV})\end{array}$ & $\begin{array}{c}\left(1 \mathrm{~b}_{1}\right),\left(3 \mathrm{a}_{1}\right),\left(1 \mathrm{~b}_{2}\right) \\
\text { peak positions in } \\
\left.\text { UPS (eV below } E_{F}\right)\end{array}$ & $\begin{array}{r}q_{s t} \\
(\mathrm{~kJ} / \\
\mathrm{mol}) \\
\end{array}$ & $\begin{array}{c}v_{n} \\
\text { from kinetic } \\
\text { fit } \\
\end{array}$ & $\begin{array}{c}n \\
\text { from } \\
\text { kinetic fit }\end{array}$ & $\begin{array}{c}E_{\text {des }} \\
(\mathrm{kJ} / \mathrm{mol})\end{array}$ \\
\hline $\mathrm{FeO}(111)$ & $\begin{array}{c}\beta_{I} \\
\text { (monom.) }\end{array}$ & $0.55 \pm 0.03$ & 1.8 & $-5.9,-8.2,-12.2$ & $\overline{52 \pm 2}$ & $\begin{array}{c}3 \pm 2 \times 10^{15} \\
\mathrm{~s}^{-1}\end{array}$ & $\begin{array}{l}1, \text { mobile } \\
\text { prec., } K= \\
0.13 \pm 0.04\end{array}$ & $52)^{\mathrm{a}}$ \\
\hline$\overline{\mathrm{FeO}(111)}$ & $\begin{array}{c}\beta_{2} \\
\text { (bilayer) }\end{array}$ & $1.3 \pm 0.2$ & 1.5 & $-6.1,-8.7 /-9.6,-12.6$ & $47 \pm 2$ & $\begin{array}{c}3 \times 10^{(15 \pm 1)} \\
\mathrm{s}^{-1}\end{array}$ & $\begin{array}{l}1, \text { mobile } \\
\text { prec., } K= \\
0.08 \pm 0.05\end{array}$ & - \\
\hline $\mathrm{FeO}(111)$ & $\alpha$ (ice) & $>1.3$ & 1.3 & $-6.4,-10.0,-12.7$ & $40) \mathrm{b}$ & & & $48)^{\mathrm{c}}$ \\
\hline $\mathrm{Fe}_{3} \mathrm{O}_{4}(111)$ & $\gamma(\mathrm{OH}+\mathrm{H})$ & $0.43 \pm 0.03$ & & $-5.8(1 \pi),-9.8(3 \sigma)$ & $65 \pm 2$ & $\begin{array}{c}(2.4 \pm 1) \times \\
10^{-6} \mathrm{~cm}^{-2} \mathrm{~s}^{-1}\end{array}$ & 2 & $50 \pm 10)^{\mathrm{d}}$ \\
\hline $\mathrm{Fe}_{3} \mathrm{O}_{4}(111)$ & $\begin{array}{c}\beta_{I} \\
\text { (monom.) }\end{array}$ & $0.86 \pm 0.03$ & 1.9 & $-6.1,-8.3,-12.1$ & $50)^{\mathrm{e}}$ & $\begin{array}{c}10^{9}-10^{14} \\
(\Theta-\text { depend. })^{\mathrm{e}}\end{array}$ & 1 & $49)^{\mathrm{f}}$ \\
\hline $\mathrm{Fe}_{3} \mathrm{O}_{4}(111)$ & $\alpha$ (ice) & $>0.86$ & 1.3 & $-6.5,-9.6,-12.5$ & $38)^{b}$ & & & $48)^{\mathrm{c}}$ \\
\hline$\alpha-\mathrm{Fe}_{2} \mathrm{O}_{3}(0001)$ & $\gamma$ & $\approx 0.5)^{\mathrm{g}}$ & - & - & - & & & $63)^{h}$ \\
\hline$\alpha-\mathrm{Fe}_{2} \mathrm{O}_{3}(0001)$ & $\beta$ & $\approx 1)^{g}$ & - & - & - & & & $52)^{h}$ \\
\hline$\alpha-\mathrm{Fe}_{2} \mathrm{O}_{3}(0001)$ & $\alpha$ (ice) & $>1)^{\mathrm{g}}$ & - & - & - & & & $48)^{c}$ \\
\hline
\end{tabular}

)$^{\mathrm{a}}$ Assuming first-order desorption and $v_{1}=3 \times 10^{15} \mathrm{~s}^{-1}$, the value deduced from the kinetic fit.

)$^{\mathrm{b}}$ Lower limit.

)$^{c}$ From direct analysis of the Polanyi-Wigner equation using $v_{1}=4 \times 10^{15} \mathrm{~s}^{-1}$ from [357].

)$^{d}$ From TDS peak shift analysis assuming second-order, which also yields $v_{2}=10^{-(5 \pm 2)} \mathrm{cm}^{-2} \mathrm{~s}^{-1}$.

)$^{\text {e }}$ Assuming first-order desorption with constant $\mathrm{q}_{\mathrm{st}}$ and fitting the frequency factor $\mathrm{v}_{1}$.

)$^{\mathrm{f}}$ Assuming first-order desorption and $v_{1}=10^{13} \mathrm{~s}^{-1}$, the mean value deduced from the kinetic fit.

)$^{\mathrm{g}}$ estimated values.

)$^{\mathrm{h}}$ Redhead analysis, assuming $\mathrm{v}_{1}=10^{13} \mathrm{~s}^{-1}$.

The sticking coefficient of water is close to unity on all Fe-oxide surfaces investigated. The isosteric heats of adsorption $q_{s t}$ and the desorption energies $E_{d e s}$ of the different species were determined quantitatively and are listed in Tables 6.3 and 6.4. They have the same order of magnitude as those measured on oxide powders $(40-60 \mathrm{~kJ} / \mathrm{mol})$ [58]. Although the formation of $\mathrm{OH}$ and $\mathrm{H}$ species suggests a specific chemical interaction with the surface, the corresponding $q_{s t}$ value is not very much higher $(65 \mathrm{~kJ} / \mathrm{mol})$ than for the species physisorbed on $\mathrm{O}$ sites on $\mathrm{Fe}_{3} \mathrm{O}_{4}(111)$ and $\mathrm{FeO}(111)$ (50$52 \mathrm{~kJ} / \mathrm{mol}$ ). Nevertheless, high equilibrium coverages for dissociated water are established, even beyond room temperature. This is due to the slow recombinative desorption of water, which is of second-order and has an extremely low frequency factor. The capability of surface Fe sites to dissociate water suggests that water could provide reactive $\mathrm{O}$ or $\mathrm{OH}$ species that can take part in an oxy-dehydrogenation mechanism of ethylbenzene to styrene over Fe-oxide based catalysts. 


\subsection{Styrene adsorption}

The TDS traces for styrene desorption from the three Fe-oxide surfaces are shown in Fig. 6.22. As for $\mathrm{EB}$ and water, only physisorbed and condensed species are observed on $\mathrm{FeO}(111)$, whereas additional chemisorbed species are observed on the $\mathrm{Fe}_{3} \mathrm{O}_{4}(111)$ and $\alpha-\mathrm{Fe}_{2} \mathrm{O}_{3}(0001)$ surfaces. On all films, desorption energies around $50 \mathrm{~kJ} / \mathrm{mol}$ and $43 \mathrm{~kJ} / \mathrm{mol}$ are obtained for the physisorbed $\beta$ species and condensed $\alpha$ species, respectively.
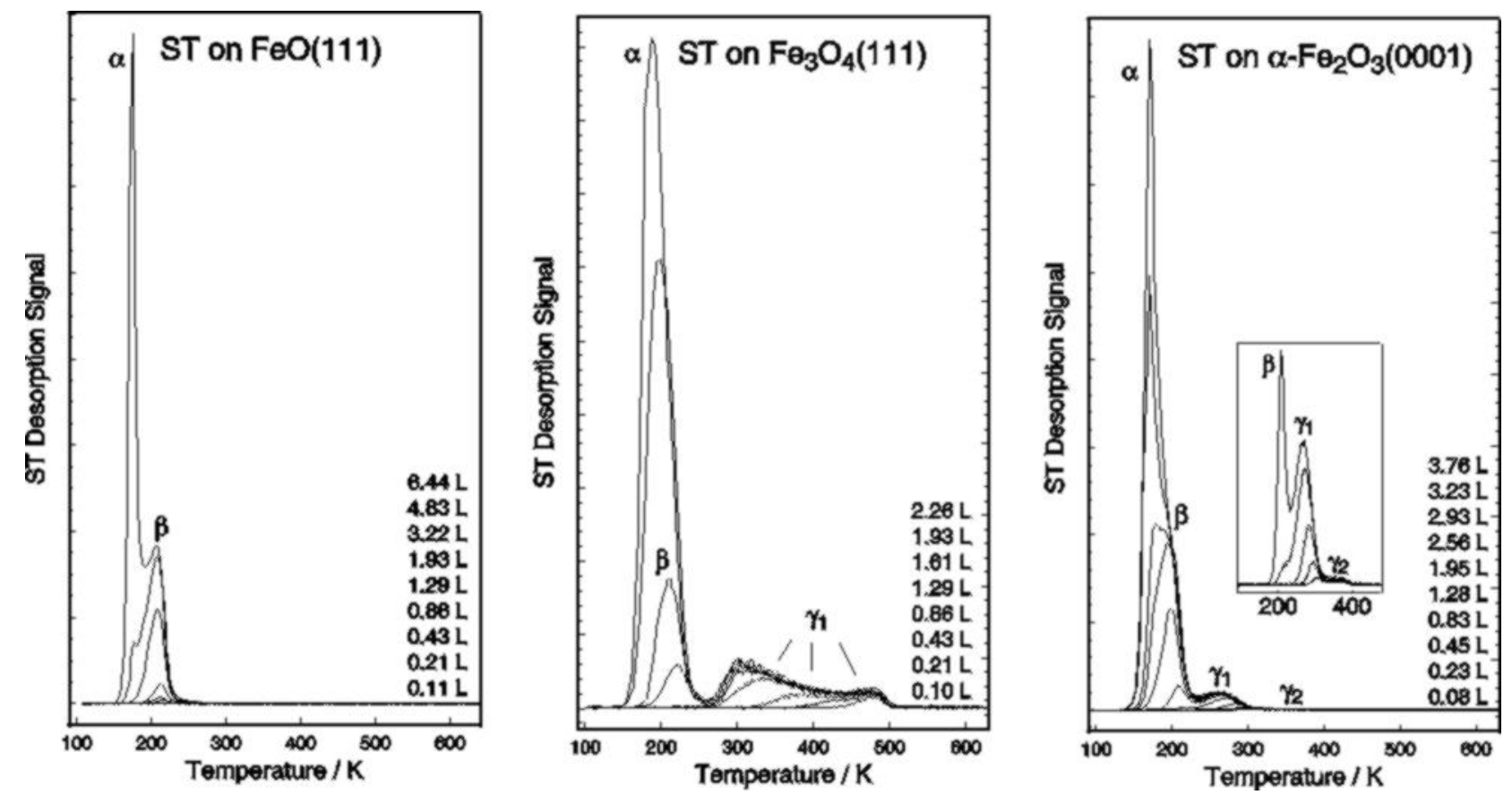

Fig. 6.22. Thermal desorption spectra of styrene adsorbed onto epitaxial Fe-oxide films grown onto $\mathrm{Pt}(111)$ : (a) 1-2 ML thick $\mathrm{FeO}(111)$ film, (b) $\mathrm{Fe}_{3} \mathrm{O}_{4}(111)$ and (c) $\alpha-\mathrm{Fe}_{2} \mathrm{O}_{3}(0001)$ multilayer films. Exposures are given in units of Langmuir $\left(1 \mathrm{~L}=1.33 \times 10^{-6} \mathrm{mbar} \cdot \mathrm{s}\right)$. After $[365,366]$.

On the $\mathrm{Fe}_{3} \mathrm{O}_{4}(111)$ surface, the chemisorbed $\gamma$ species starts to desorb at about $500 \mathrm{~K}$. This desorption temperature of the most strongly bound styrene species is slightly higher than for chemisorbed $\mathrm{EB}$ on $\mathrm{Fe}_{3} \mathrm{O}_{4}(111)(\mathrm{T}=450 \mathrm{~K})$, resulting in a higher desorption energy for styrene $(118 \mathrm{~kJ} / \mathrm{mol})$ than for EB $(91 \mathrm{~kJ} / \mathrm{mol})$. After saturation of this signal, a second desorption peak evolves around $\mathrm{T}=300 \mathrm{~K}$, resulting in a very broad chemisorption signal, extending from $280-500 \mathrm{~K}$ that we denote $\gamma$. Upon further exposure, the pysisorbed $\beta$ and condensed $\alpha$ signals evolve, which are not well separated in this case.

On the $\mathrm{Fe}_{2} \mathrm{O}_{3}(0001)$ surface, first a chemisorbed $\gamma_{2}$ signal around $\mathrm{T}=400 \mathrm{~K}$ evolves. This chemisoprtion signal appears at the same temperature as the $\gamma$ signal for low EB coverage on $\mathrm{Fe}_{2} \mathrm{O}_{3}$. It saturates at small exposures around $0.1 \mathrm{~L}$. The $\gamma_{1}$ signal then evolves with a desorption maximum shifting from $T=295 \mathrm{~K}$ to $275 \mathrm{~K}$. These temperatures are also slightly higher than for the corresponding $\gamma$ main signal of EB. It corresponds to an initial desorption energy of $73 \mathrm{KJ} / \mathrm{mol}$, about $10 \mathrm{KJ} / \mathrm{mol}$ higher than for EB. Upon further exposure, the physisorbed $\beta$ and condensed $\alpha$ signals evolve.

The chemisorbed and physisorbed species saturate around exposures of $2 \mathrm{~L}$, indicating sticking coefficients close to unity, as for styrene. Both on $\mathrm{Fe}_{3} \mathrm{O}_{4}$ and $\mathrm{Fe}_{2} \mathrm{O}_{3}$ the dominating $\gamma$ chemisorption states of styrene are slightly stronger bound than the corresponding EB species. The more strongly 
bound $\gamma_{2}$ state on the $\mathrm{Fe}_{2} \mathrm{O}_{3}$ surfaces, which sometimes is also found on $\mathrm{Fe}_{3} \mathrm{O}_{4}$, has a saturation coverage at least 10 times lower than the dominating $\gamma_{1}$ chemisorption state.

Styrene adsorption has been studied by NEXAFS on the three Fe-oxide films [333, 358]. The condensed phase spectrum of St is contained in Fig. 6.23. The assignment of the spectral features was given in Section 6.1.4. The spectrum of St is very similar to that of EB. The only clear difference is a shoulder at the low energy side of the main $\pi^{*}$ resonance in the St spectra (A' in Fig. 6.12), which originates from the vinyl group [333]. As for EB, the NEXAFS spectra of St display a clear dependence on the orientation of the $E$-vector of the incident light with respect to the surface. Figure 6.23 compares the normal and glancing incidence spectra for coverages well below saturation of the first adsorbing species, which is physisorbed $\beta$-St on $\mathrm{FeO}(111)$ and chemisorbed $\gamma$-St on $\mathrm{Fe}_{3} \mathrm{O}_{4}(111)$ and $\alpha-\mathrm{Fe}_{2} \mathrm{O}_{3}(0001)$. The tilt angle $\alpha$ of the plane of the phenyl group with respect to the surface can be deduced from the difference of the $\pi^{*}$ intensities and is given in Fig. 6.23 As for EB, the phenyl group of St physisorbed on $\mathrm{FeO}(111)$ it is strongly tilted, whereas St chemisorbed on $\mathrm{Fe}_{3} \mathrm{O}_{4}(111)$ and $\alpha$ $\mathrm{Fe}_{2} \mathrm{O}_{3}(0001)$ lies fairly flat on the surface $\left(\alpha=23\right.$ to $\left.28^{\circ}\right)$. It should be emphasized again that these tilt angles should be taken as qualitative, since the disturbance of bond lengths as well as $\mathrm{C}-\mathrm{H}$ and $\mathrm{C}-\mathrm{C}$ bond angles may contribute to the observed non-zero $\pi *$ intensity for normal light intensity as discused in Section 6.1.4. In the case of St, the intensity from the vinyl group also contributes to the $\pi *$ intensity (shoulder A'). In the gas-phase, the phenyl and vinyl groups should be in plane, but the energy needed for rotation of the vinyl group is quite low, so that the orientation of the vinyl group may deviate from that of the phenyl group in the chemisorbed state. Unfortunately, the noise level in Fig. 6.23 does not allow us to separate the intensities at normal incidence into contributions from the phenyl and vinyl groups.

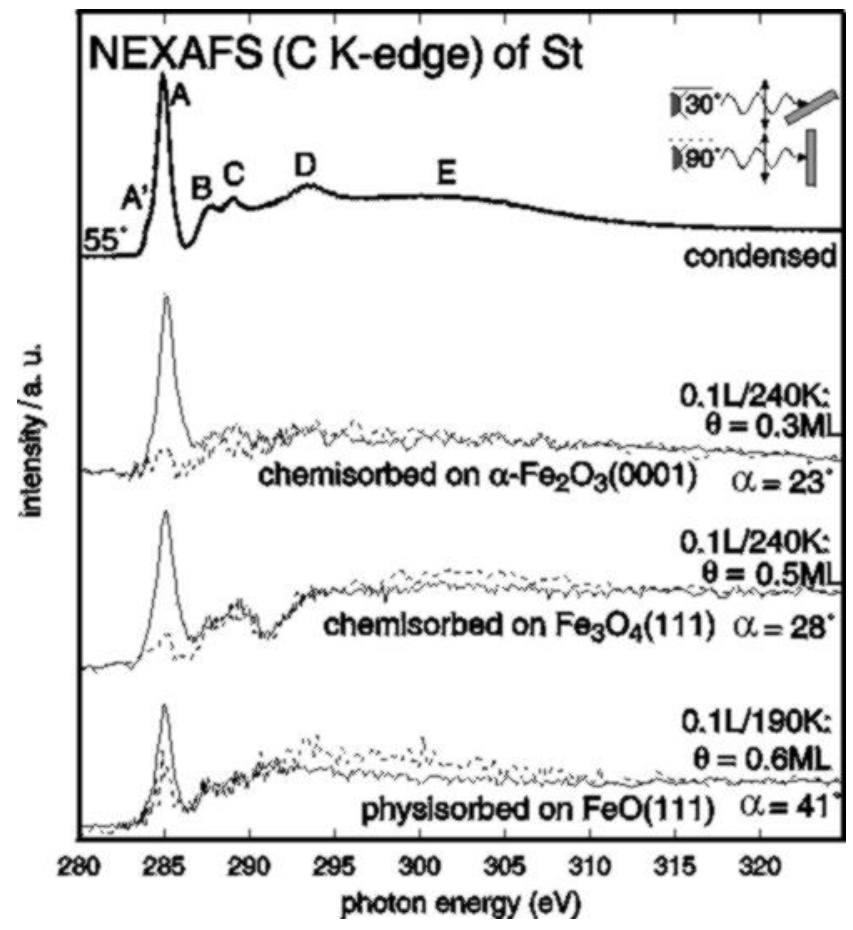

Fig. 6.23. C K-edge NEXAFS spectra of styrene layers on different $\mathrm{Fe}$-oxides in submonolayer regime. Full lines: grazing incidence $\left(30^{\circ}\right)$; dashed: normal incidence $\left(90^{\circ}\right)$. Exposure conditions (doses and temperatures), resulting coverages $(\theta)$ and calculated tilt angles between molecular plane and surfaces $(\alpha)$ are indicated. From [358].

\subsection{Summary}

The thermodynamic and kinetic data for the physisorption and chemisorption of water, EB and St on the three oxide films $\mathrm{FeO}(111), \mathrm{Fe}_{3} \mathrm{O}_{4}(111)$ and $\mathrm{Fe}_{2} \mathrm{O}_{3}(0001)$ are summarized in Table 6.4. There are certain similarities in the behaviour of all the three adsorbates. Only physisorbed states are 
observed on the $\mathrm{FeO}(111)$ film, whereas a chemisorbed state precedes physisorption on both $\mathrm{Fe}_{3} \mathrm{O}_{4}$ and $\mathrm{Fe}_{2} \mathrm{O}_{3}$. The values of $q_{s t}$ and $E_{\text {des }}$ of the physisorbed states of all adsorbates are similar, but the binding energies of the chemisorbed species differ more strongly. Both EB and St are bound more strongly on $\mathrm{Fe}_{3} \mathrm{O}_{4}(111)$ than on $\mathrm{Fe}_{2} \mathrm{O}_{3}(111)$ and water even dissociates on $\mathrm{Fe}_{3} \mathrm{O}_{4}(111)$.
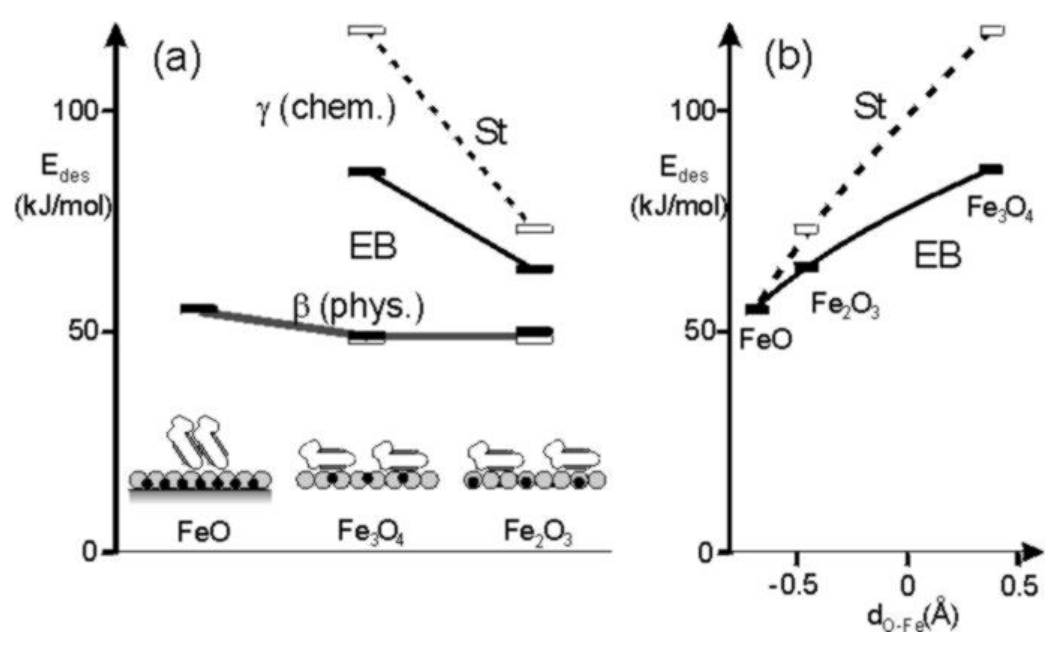

Fig. 6.24. (a). Desorption energies $E_{\text {des }}$ of physisorbed and chemisorbed EB and St on the three oxide films $\mathrm{FeO}(111), \mathrm{Fe}_{3} \mathrm{O}_{4}(111)$ and $\mathrm{Fe}_{2} \mathrm{O}_{3}(0001)$ from TDS (Table 6.4), adsorbate arrangement of initially adsorbing species at low coverage (schematic, shown for EB) from NEXAFS [358] and substrate structures from photoelectron diffraction ( $\mathrm{FeO},[260])$ and LEED $\left(\mathrm{Fe}_{3} \mathrm{O}_{4}\right.$ [284] and $\mathrm{Fe}_{2} \mathrm{O}_{3}$

[294]) The O-Fe layer distances (negative for $\mathrm{Fe}$-layer below O-layer) $d_{O-F e}$ are $-0.68 \AA$ for $\mathrm{FeO}$ (structure $\mathrm{S} 2$, Table 5.2), $-0.46 \AA$ for $\mathrm{Fe}_{2} \mathrm{O}_{3}$ (best-fit structure, see Section 5.6.2) and +0.38 $\AA$ for $\mathrm{Fe}_{3} \mathrm{O}_{4}$, Fig. 5.29). (b). $E_{d e s}$ vs. $d_{O-}$ $\mathrm{Fe}$ for the species adsorbing first on the three oxide substrates.

This is illustrated for EB and St in Fig. 6.24, which correlates the values of $E_{d e s}$, deduced from TDS, with the adsorbate orientations deduced from NEXAFS and the substrate structure deduced from LEED and photoelectron diffraction. In Fig. 6.24b, $E_{\text {des }}$ of the initially adsorbed species (physisorbed on $\mathrm{FeO}$, chemisorbed on $\mathrm{Fe}_{3} \mathrm{O}_{4}$ and $\mathrm{Fe}_{2} \mathrm{O}_{3}$ ) is plotted vs. the position of the first Fe layer with respect to the first O-layer of the relaxed substrate. $E_{d e s}$ increases monotonously with the position of the Felayer relative to the O-layer, which illustrates the importance of interaction with acidic Fe for chemisorption. The physisorption strength on $\mathrm{FeO}$ is about $55 \mathrm{~kJ} / \mathrm{mol}$. No specific interaction with the substrate occurs, the adsorbate-adsorbate interaction exceeds the adsorbate-substrate interaction and the molecules are strongly tilted. On the other substrates, the adsorbate-substrate interaction prevails and the molecules ly flat on the surfaces. Interesting is the case of $\mathrm{Fe}_{2} \mathrm{O}_{3}$, where $E_{d e s}$ for $\mathrm{EB}$ is only slightly higher than for physisorption on $\mathrm{FeO}$. The interaction with $\mathrm{Fe}$ on $\mathrm{Fe}_{2} \mathrm{O}_{3}$ is obviously just strong enough to overbalance the adsorbate-adsorbate interaction in the tilted arrangement of physisorption and to align the molecules flat on the surface. The acid-base interaction between Fe and adsorbates may thus also become effective if the atomic plane of the Fe-atoms is below the O-layer. This is related to the fact that electronic bulk states near the Fermi edge origin mainly from Fe-atoms (see Section 5.2.3). Calculations for $\mathrm{Fe}_{2} \mathrm{O}_{3}$ showed that surface states on the realaxed surface are $\mathrm{Fe}$ derived [182]. On $\mathrm{Fe}_{2} \mathrm{O}_{3}$ with $d_{O-F e}=-0.46 \AA$, Fe-states extend through the top O-layer and can interact with the adsorbates. On the $\mathrm{FeO}$ film with $d_{O-F e}=-0.68 \AA$, the Fe-layer lies too deep so that the interaction is weaker than the combined van der Waals forces of adsorbate-adsorbate and adsorbatesubstrate interaction.

The EB molecules in Fig. 6.24a are plotted with the gas phase structure [359], i.e., with the ethyl group bent out of the plane of the phenyl group. This structure may be modified in the adsorbed state. The lowest energy structure of St in the gas phase is flat with the $\pi$-orbitals both of the phenyl- and the vinyl-group forming a common conjugated system, but bending of the vinyl-group does not cost much 
energy [360]. That $\mathrm{St}$ is chemisorbed more strongly than $\mathrm{EB}$ on $\mathrm{Fe}_{3} \mathrm{O}_{4}(111)$ and $\mathrm{Fe}_{2} \mathrm{O}_{3}(0001)$, suggests a more extended $\pi$-system in St with the phenyl- and the vinyl-group in one plane

TABLE 6.4. Compilation of thermodynamic and kinetic data for water, EB and St adsorption on $\mathrm{FeO}(111), \mathrm{Fe}_{3} \mathrm{O}_{4}(111)$ and $\mathrm{Fe}_{2} \mathrm{O}_{3}(111)$ surfaces. For further details see Tables 6.2, 6.3.

\begin{tabular}{|c|c|c|c|c|c|c|c|c|c|}
\hline \multirow{2}{*}{$\begin{array}{l}\text { Sub- } \\
\text { strate }\end{array}$} & \multirow[t]{2}{*}{ Ads. } & \multicolumn{4}{|c|}{ Chemisorbate $\gamma$} & \multicolumn{4}{|c|}{ Physisorbate $\beta$} \\
\hline & & $\begin{array}{c}q_{s t} \\
(\mathbf{C C})\end{array}$ & $\begin{array}{c}\nu, n \\
\text { (kin. fit) }\end{array}$ & $\begin{array}{c}E_{d e s} \\
\text { (TDS) }\end{array}$ & $\begin{array}{c}v, n \\
\text { (TDS) }\end{array}$ & $\begin{array}{c}q_{s t} \\
(\mathbf{C C})\end{array}$ & $\begin{array}{c}v, n \\
\text { (kin. fit) }\end{array}$ & $\begin{array}{c}E_{d e s} \\
(\text { TDS }) \\
\end{array}$ & $\begin{array}{c}v, n \\
\text { (TDS) }\end{array}$ \\
\hline \multirow{4}{*}{$\begin{array}{c}\mathrm{FeO} \\
(111)\end{array}$} & \multirow[t]{2}{*}{$\mathrm{H}_{2} \mathrm{O}$} & \multirow[t]{2}{*}{--} & \multirow[t]{2}{*}{--} & \multirow[t]{2}{*}{--} & \multirow[t]{2}{*}{--} & $52\left(\beta_{1}\right)$ & \multirow{2}{*}{$\begin{array}{l}\left.3 \times 10^{15}\right) \\
\left(\beta_{1}\right), 1 / 1\end{array}$} & \multirow{2}{*}{$\begin{array}{l}52 \\
(\mathrm{R})\end{array}$} & \multirow{2}{*}{$\begin{array}{l}3 \times 10^{15} \\
(\mathrm{~A}), 1\end{array}$} \\
\hline & & & & & & $47\left(\beta_{2}\right)$ & & & \\
\hline & EB & -- & -- & -- & -- & 58 & $\begin{array}{l}4.8 \times 10^{14}, \\
1 / 1\end{array}$ & $\begin{array}{l}55 \\
(\mathrm{LE})\end{array}$ & $\begin{array}{l}5 \times 10^{12}, \\
1\end{array}$ \\
\hline & St & -- & -- & -- & -- & 55 & $\begin{array}{l}3 \times 10^{10} \\
2 / 1\end{array}$ & & \\
\hline \multirow[t]{3}{*}{$\begin{array}{c}\mathrm{Fe}_{304} \\
(111)\end{array}$} & $\mathrm{H}_{2} \mathrm{O}$ & 65 & $\begin{array}{l}2.4 \times 10^{-6} \\
\mathrm{~cm}^{2} \mathrm{~s}^{-1} \\
2 / 2\end{array}$ & $50 \pm 10$ & $\begin{array}{l}10^{-(5 \pm 2)} \\
\mathrm{cm}^{2} \mathrm{~s}^{-1} \\
2\end{array}$ & 50 & $\begin{array}{l}10^{9}-10^{14} \\
1 / 1\end{array}$ & $\begin{array}{l}49 \\
(\mathrm{R})\end{array}$ & $10^{13}$ \\
\hline & EB & $94-74$ & $\begin{array}{l}5 \times 10^{12}- \\
2 \times 10^{10} \\
1 / 1\end{array}$ & $\begin{array}{l}86 \\
(\mathrm{LE})\end{array}$ & $\begin{array}{l}1 \times 10^{12} \\
1\end{array}$ & $65-52$ & $\begin{array}{l}10^{13}- \\
5 \times 10^{15} \\
1 / 1(\mathrm{NE})\end{array}$ & $\begin{array}{l}47 \\
(\mathrm{LE}) \\
(\mathrm{NE})\end{array}$ & $\begin{array}{l}8 \times 10^{11}, \\
1\end{array}$ \\
\hline & $\mathrm{St}$ & (Frag) & & $\begin{array}{l}118 \\
\text { (LE) }\end{array}$ & $3 \times 10^{11}$ & & & $\begin{array}{l}46 \\
(\mathrm{LE})\end{array}$ & $\begin{array}{l}6 \times 10^{10}, \\
1\end{array}$ \\
\hline \multirow[t]{3}{*}{$\begin{array}{c}\alpha-\mathrm{Fe}_{2} \mathrm{O}_{3} \\
(0001)\end{array}$} & $\mathrm{H}_{2} \mathrm{O}$ & & & $\begin{array}{l}63 \\
(\mathrm{R})\end{array}$ & $10^{13}$ & & & $\begin{array}{l}52 \\
(\mathrm{R})\end{array}$ & $10^{13}$ \\
\hline & EB & & & $\begin{array}{l}64 \\
(\mathrm{LE}) \\
\end{array}$ & $1 \times 10^{12}$ & & & $\begin{array}{l}50 \\
(\mathrm{LE}) \\
\end{array}$ & $\begin{array}{l}1 \times 10^{11}, \\
1\end{array}$ \\
\hline & $\mathrm{St}$ & & & $\begin{array}{l}73 \\
\text { (LE) }\end{array}$ & $5 \times 10^{12}$ & & & $\begin{array}{l}48 \\
(\mathrm{LE})\end{array}$ & $\begin{array}{l}4 \times 10^{10} \\
1\end{array}$ \\
\hline
\end{tabular}

$q_{s t}$ : Isosteric heat of adsorption. Energies in $\mathrm{kJ} / \mathrm{mol} ; \mathrm{v}$ in $\mathrm{s}^{-1}$, if not stated otherwise. (CC): From

Clausius-Clapeyron analysis of isobars. (kin. fit): From kinetic fit of isobars; reaction order $n$ for adsorption and desorption is given. (TDS) from analysis of thermal desorption data; reaction order for desorption is given. (R): Redhead method, $1^{\text {st }}$ order, $v$ assumed. (LE): Leading edge method; large uncertainty in $v$. (NE): Ads.-des. equilibrium not established; data uncertain. (A): Value for $v$ taken from kin. fit analysis. (Frag): Observation of partial adsorbate fragmentation and coke formation; measurement in ads.-des- equilibrium not possible.

\section{Reactivity studies}

As reviewed in Section 4, the technical dehydrogenation of ethylbenzene to styrene is performed over Fe-oxide based catalysts, in the presence of steam. The most active catalysts are potassium promoted, but also unpromoted $\alpha-\mathrm{Fe}_{2} \mathrm{O}_{3}(0001)$ shows activity. Therefore, the single-crystalline $\mathrm{Fe}$ oxide films represent model systems that allow us to study correlations between the oxide 
stoichiometry, surface structure and the catalytic activity. For this purpose, $\mathrm{Fe}_{3} \mathrm{O}_{4}(111)$ and $\alpha-\mathrm{Fe}_{2} \mathrm{O}_{3}(0001)$ multilayer films with different surface defect concentrations were prepared. The defect concentrations were deduced qualitatively from the diffuse background intensities in the LEED patterns and from the FWHM of the diffraction spots. All experiments were performed under in-situ conditions. After characterization in UHV, the model catalyst films were transferred into the reaction cell, as discussed in Section 3. Post-reaction analysis was also performed in-situ, after transferring the samples back into the UHV analysis chamber.

\section{$\mathrm{Fe}_{2} \mathrm{O}_{3}$ \\ LEED before}

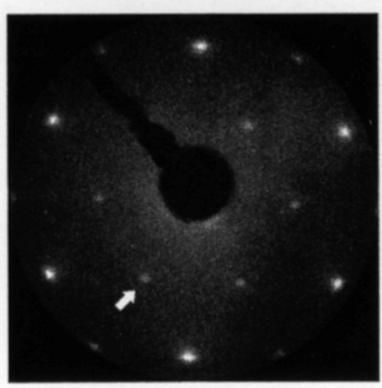

$$
\begin{aligned}
& \mathrm{I}_{\mathrm{B}} / \mathrm{I}_{\max }=0.37 \\
& \mathrm{FWHM}=9.6 \%
\end{aligned}
$$

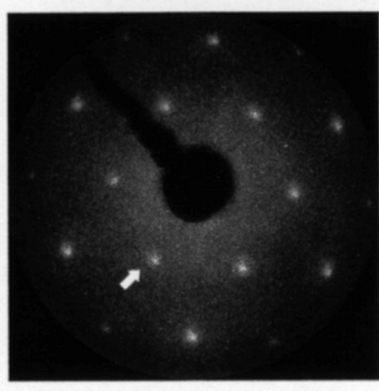

$\mathrm{I} / I_{\max }=0.53$

$\mathrm{FWHM}=13.5 \%$

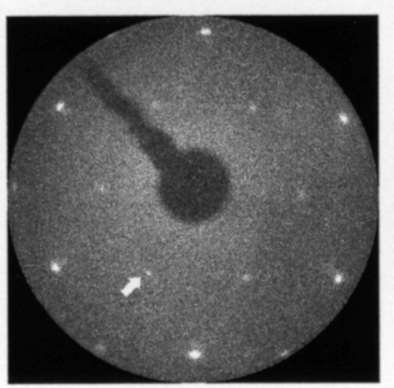

$\mathrm{I} / \mathrm{I} / \max =0.68$

$\mathrm{FWHM}=15.8 \%$

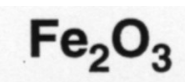

PEEM after
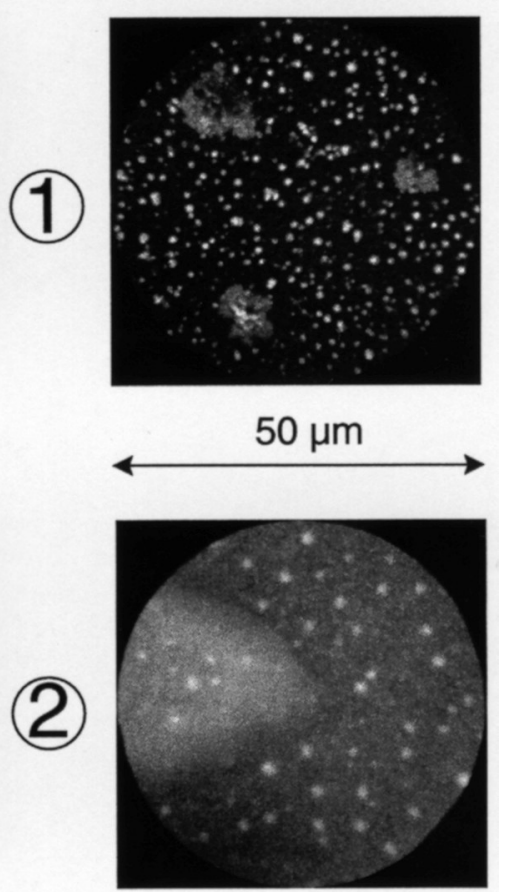

$50 \mu \mathrm{m}$

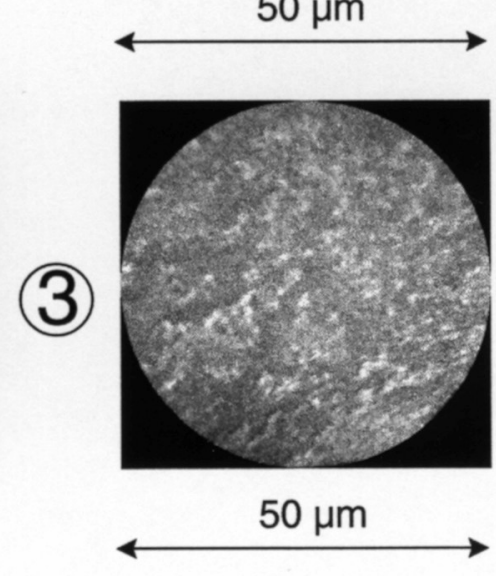

Fig. 7.1. Left: LEED patterns of three $\mathrm{Fe}_{2} \mathrm{O}_{3}(0001)$ films taken at $E=60 \mathrm{eV}$ before reaction experiments. For each pattern FWHM of (10) beam (indicated by arrows) and ratio between diffuse background intensities $I_{B}$ and intensities in (10) beam maximum $I_{\max }$ is given. Right: $50 \mu \mathrm{m}$ diameter PEEM images observed on three films after reaction experiment. From [367].

\subsection{Reaction studies at low pressures}

Stationary reaction experiments were performed, first under vacuum conditions in a water to EB mixture of 5:1 with a total gas pressure of $3 \times 10^{-6}$ mbar at $T=873 \mathrm{~K}$. The sample was placed in front of the mass spectrometer, in order to detect the reaction products. No reproducible styrene formation 
could be observed under these conditions on any of the films investigated. However, carbonaceous surface deposits formed during these experiments. Comparison of PEEM images from $\mathrm{Fe}_{3} \mathrm{O}_{4}(111)$ and $\alpha-\mathrm{Fe}_{2} \mathrm{O}_{3}(0001)$ films, taken before and after the reaction experiments, show that bright spots develop during reaction. Much more of them form on $\mathrm{Fe}_{2} \mathrm{O}_{3}$. These bright spots are created by carbonaceous deposits as deduced from AES and temperature programmed reaction (TPR) measurements, such as the one shown below in Fig. 7.5. Clearly, more EB has decomposed on $\mathrm{Fe}_{2} \mathrm{O}_{3}$, when compared to $\mathrm{Fe}_{3} \mathrm{O}_{4}$, which might have been accompanied by a styrene formation too small to be detectable.

\subsection{Reaction studies at elevated pressures}

\subsubsection{Batch-reactor experiments}

The catalytic film activities were investigated in batch-rector experiments in a water-EB mixture of 10:1 and a total gas pressure of $0.6 \mathrm{mbar}$, as described in Section 3. These conditions approximate the real situation of the technical styrene synthesis reaction. On the left side of Fig. 7.1, the LEED patterns of three $\mathrm{Fe}_{2} \mathrm{O}_{3}(0001)$ films are shown before the reaction experiments were performed. The diffuse background intensities in these LEED patterns were quantified by the ratio between the intensity $\mathrm{I}_{\max }$ in the maximum of the (10) LEED spot indicated by the arrows and the background intensity $\mathrm{I}_{B}$ measured between the main diffraction spots [258]. The FWHM of the (10) beam is quantified in percent of the first Brillouin zone width as given by the $\mathrm{Fe}_{2} \mathrm{O}_{3}(0001)$ surface unit cell. Since the electron energy of $E=60 \mathrm{eV}$ corresponds to an intermediate scattering condition, this FWHM scales with the step densities on the surfaces [361, 362]. Film (1) has the lowest step and point defect density on the surface, film (2) has a medium and film (3) has the highest step and point defect density.

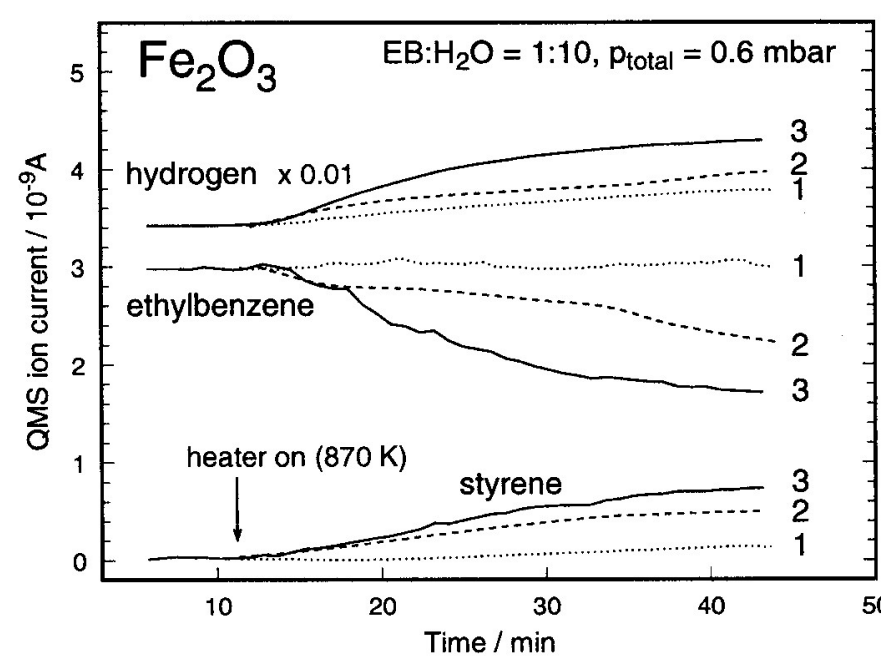

Fig. 7.2. Batch reactor experiments on epitaxial $\mathrm{Fe}_{2} \mathrm{O}_{3}(0001)$ films with low (1), medium (2) and high surface defect density (3). Initial molar ratio of ethylbenzene:water was 1:10 and total pressure 0.6 mbar. QMS intensities for molecular masses of $\mathrm{H}_{2}$ (2), $\mathrm{EB}(106)$ and styrene(104) are displayed as function of time. An offset was added to $\mathrm{H}_{2}$ signal for better presentation. From [367].

Figure 7.2 shows the results of the batch-reactor experiments performed on these films. After admission of the educts, the samples were heated to the reaction temperature of $870 \mathrm{~K}$, which was reached after $12 \mathrm{~min}$, as indicated by the arrow. For the low defect density $\mathrm{Fe}_{2} \mathrm{O}_{3}$ film (1), no significant changes in the EB and styrene signals are observed, indicating negligible catalytic activity. Only the $\mathrm{H}_{2}$ signal increases slightly. On the films with medium high defect density $(2,3), \mathrm{H}_{2}$ and styrene formation is stronger and the EB concentration decreases. Formation of styrene and $\mathrm{H}_{2}$ and consumption of EB, thus, scales with the surface defect concentration. After about 30 min reaction 
time, the active films (2) and (3) were deactivated and the styrene and $\mathrm{H}_{2}$ product concentrations do not increase significantly anymore.

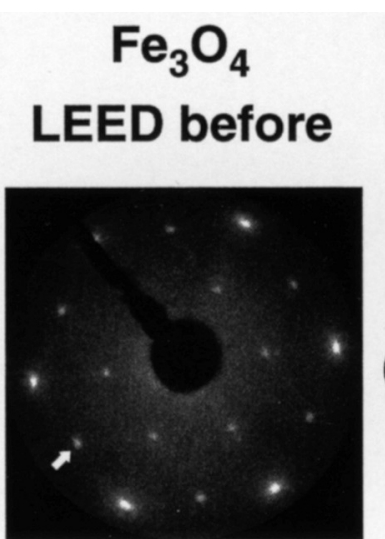

$$
\begin{aligned}
& \mathrm{I}_{\mathrm{B}} / \mathrm{I}_{\max }=0.26 \\
& \mathrm{FWHM}=7.5 \%
\end{aligned}
$$

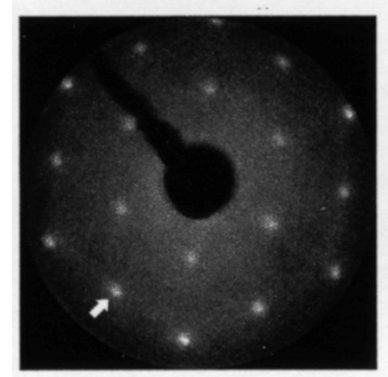

$\mathrm{I} / \mathrm{I} / \max =0.43$

$\mathrm{FWHM}=11.7 \%$

\section{$\mathrm{Fe}_{3} \mathrm{O}_{4}$}

PEEM after

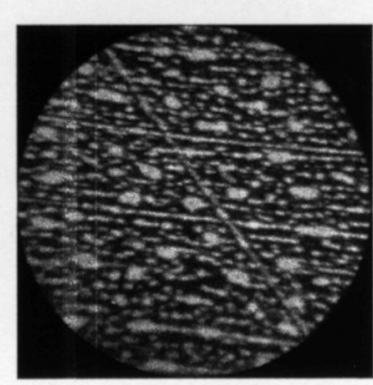

$50 \mu \mathrm{m}$
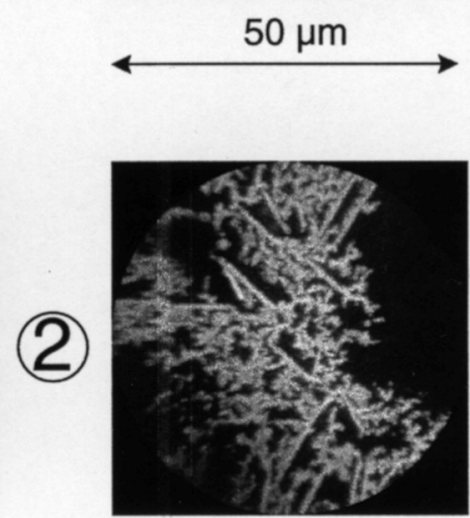

$50 \mu \mathrm{m}$

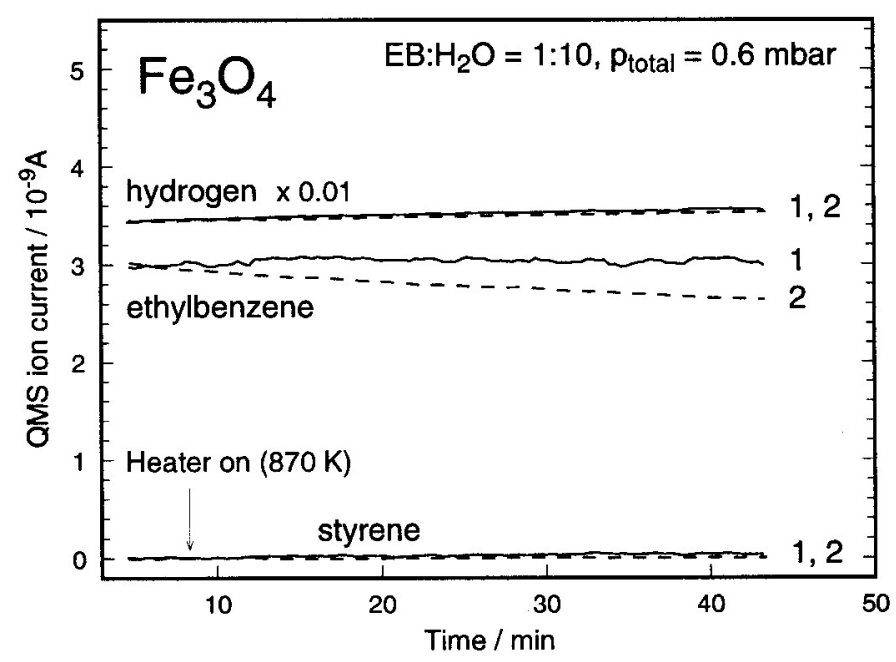

Fig. 7.3. Left: LEED patterns of two $\mathrm{Fe}_{3} \mathrm{O} 4(111)$ films taken at $\mathrm{E}=60 \mathrm{eV}$ before reaction experiment. FWHM of (10) beam and background to (10) beam intensity $I_{B} / I_{\max }$ is given. Right: 50 $\mu \mathrm{m}$ diameter PEEM images after reaction experiment. From [367].
Fig. 7.4. Batch-reactor experiments on epitaxial $\mathrm{Fe}_{3} \mathrm{O} 4(111)$ films with low (1) and high surface defect density (2). Conditions as in Fig. 7.2. From [367].

Corresponding experiments were performed on $\mathrm{Fe}_{3} \mathrm{O}_{4}$ films with low and high defect density. Figure 7.3 shows their LEED patterns and Fig. 7.4 the batch-reactor experiments performed on them. No catalytic styrene formation, no $\mathrm{H}_{2}$ evolution and no significant decrease of the EB concentration are observed, independent of the surface defect concentration. 
These reaction experiments reveal clearly that atomic surface defects act as active sites for the dehydrogenation of EB to styrene on the $\alpha-\mathrm{Fe}_{2} \mathrm{O}_{3}(0001)$ model catalyst films, whereas the $\mathrm{Fe}_{3} \mathrm{O}_{4}$ phase is always inactive.

\subsubsection{Post-reaction analysis}

After the reaction experiments, the model catalyst films are covered with carbonaceous surface deposits. From the energetic position and line shape of their $\mathrm{C}_{\mathrm{KLL}}$ Auger spectra (Fig. 7.5a) it can be concluded that they are graphitic. Figure $7.5 \mathrm{~b}$ shows a typical TPR experiment performed after the reaction experiment. The $\mathrm{Fe}_{2} \mathrm{O}_{3}$ film (3) with carbonaceous overlayer was heated in $10^{-6}$ mbar $\mathrm{O}_{2}$ with a linear temperature ramp to $1000 \mathrm{~K}$. First, the evolution of water is observed, but at the temperature where the batch-reactor experiments were run $(870 \mathrm{~K})$, massive formation of $\mathrm{CO}$ and of some $\mathrm{CO}_{2}$ occurs. After these TPR experiments, the LEED patterns of $\alpha-\mathrm{Fe}_{2} \mathrm{O}_{3}(0001)$ films exhibited additional diffraction spots corresponding to $\mathrm{Fe}_{3} \mathrm{O}_{4}(111)$, indicating a partial reduction of the $\mathrm{Fe}_{2} \mathrm{O}_{3}$ films, during the batch-reactor experiments.
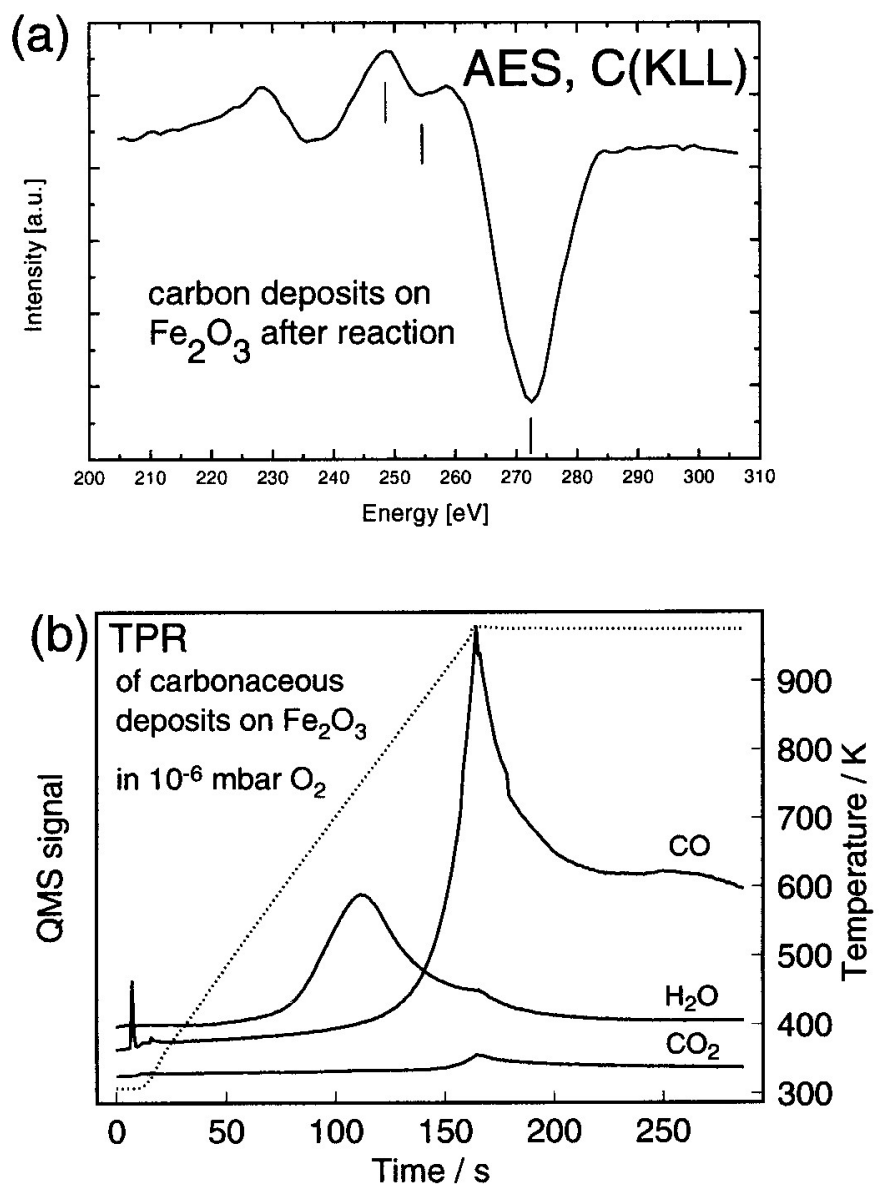

Fig. 7.5. (a) AES spectrum of $\mathrm{Fe}_{2} \mathrm{O}_{3}$ film covered by carbonaceous deposits after reaction experiment. (b) Temperature-programmed reaction experiment in $10^{-6}$ mbar $\mathrm{O}_{2}$, performed on most active $\mathrm{Fe}_{2} \mathrm{O}_{3}$ film (3) covered by a carbonaceous layer. QMS signals for molecular masses of $\mathrm{CO}, \mathrm{H}_{2} \mathrm{O}$ and $\mathrm{CO}_{2}$ are shown as a function of time. Dotted line indicates sample temperature (right ordinate scale). From [235, 367].

The hydrocarbon surface deposits were imaged with the PEEM microscope. On the low defect density $\mathrm{Fe}_{2} \mathrm{O}_{3}$ film (1) in Fig. 7.1, bright spots about $1 \mu \mathrm{m}$ in size are randomly distributed on the surface. The regions between them exhibit the same contrast as clean oxide films. Therefore, we attribute them to clean $\mathrm{Fe}_{2} \mathrm{O}_{3}$ surface regions and the bright dots to carbonaceous surface deposits. Film (2), with a medium defect density, also exhibits bright dots randomly distributed on the surface, 
but now the region between them is considerably brighter, when compared to film (1). As all PEEM images were measured under identical experimental conditions, we conclude that the regions between the bright spots are also covered with carbonaceous surface deposits. The most active $\mathrm{Fe}_{2} \mathrm{O}_{3}$ film (3) is covered by a thick carbonaceous layer after the reaction experiment, as is evident from its PEEM image with the highest brightness. This indicates that the formation of carbonaceous deposits is correlated to the catalytic activity of the films. After about $30 \mathrm{~min}$, film (2) and (3) are deactivated and completely covered with carbonaceous layers.

As on the $\mathrm{Fe}_{2} \mathrm{O}_{3}$ film (1), with a low defect density, small amounts of carbonaceous surface deposits are observed on both $\mathrm{Fe}_{3} \mathrm{O}_{4}$ films after the reaction experiments, although no styrene formation was detectable. Figure 7.3 shows that these deposits form dots and some streaky features on film (1), whereas dentritic-like structures were formed on film (2) with higher surface defect density. These dentritic structures may indicate a polymerization of the carbonaceous deposits.

\subsection{Discussion}

The kinetic parameters for the A-D processes of the molecules involved in the styrene synthesis reaction were determined quantitatively by the UHV experiments on the model catalyst surfaces. For the educt and product molecules EB and St, non-activated adsorption via weakly bound mobile precursor states into chemisorbed and physisorbed adsorption states was found, with high sticking coefficients almost upto the corresponding saturation coverages. Using the Langmuir isotherm formalism for competitive adsorption, the relative coverages of the adsorption states can be extrapolated to the technical styrene synthesis reaction conditions ( $T=900 \mathrm{~K}, p=200 \mathrm{mbar}$ ). It turns out that a negligible portion of the physisorbed states are occupied under these conditions. Table 7.1 shows the occupation extrapolated for the chemisorbed $\gamma$ states. Both EB and styrene are bound more strongly on $\mathrm{Fe}_{3} \mathrm{O}_{4}$ than on $\mathrm{Fe}_{2} \mathrm{O}_{3}$, which leads to a saturated chemisorbed phase on $\mathrm{Fe}_{3} \mathrm{O}_{4}$ and to a coverage of only about $1 / 3 \mathrm{ML}$ on $\mathrm{Fe}_{2} \mathrm{O}_{3}$. Equally important is the fact that the difference of the BEs for $\mathrm{EB}$ and $\mathrm{St}$ is larger on $\mathrm{Fe}_{3} \mathrm{O}_{4}$ than on $\mathrm{Fe}_{2} \mathrm{O}_{3}$. The relative coverages obtained from the Langmuir isotherm formalism for competitive adsorption (Table 7.1) show that the reactive sites are blocked by the styrene product on $\mathrm{Fe}_{3} \mathrm{O}_{4}\left(\Theta_{E B} / \Theta_{S t}=<0.01\right)$, whereas on $\mathrm{Fe}_{2} \mathrm{O}_{3}$ this is not the case $\left(\Theta_{E B} / \Theta_{S t} \approx 1.3\right)$. This agrees with the actual observation that $\mathrm{Fe}_{2} \mathrm{O}_{3}(0001)$ surfaces are more active for the styrene synthesis than $\mathrm{Fe}_{3} \mathrm{O}_{4}(111)$ surfaces. This result, obtained on the single-crystalline model catalyst films, confirms conclusions that were drawn from kinetic measurements over polycrystalline potassium promoted and unpromoted Fe-oxide catalysts [194]. In these reactor experiments, the styrene synthesis was studied at gas pressures in the mbar-range and it was found that the relative adsorption strength of styrene compared to EB on the sites active for dehydrogenation must be larger for the less active catalysts. Therefore, the styrene desorption was considered as the rate determining step.

However, the defect-free $\mathrm{Fe}_{2} \mathrm{O}_{3}(0001)$ surface is not active either. Defects are needed and the styrene formation increases with increasing surface defect-concentration. This clearly reveals that $\alpha-\mathrm{Fe}_{2} \mathrm{O}_{3}(0001)$ is the active Fe-oxide phase for EB dehydrogenation, but atomic defects are involved in the catalytically active sites on the unpromoted films. These defect sites may be steps, vacancies, adatoms or isolated surface atoms with a modified local electronic structure. All these types of defects are observed by STM on these surfaces. Most likely O anions with a higher basicity are exposed at the defect sites, which can deprotonate the $\mathrm{C}-\mathrm{H}$ groups of the ethyl group of the $\mathrm{EB}$ molecule. Simultaneously, or subsequently, to deprotonation, an electron must be transferred from the reaction intermediate to an acidic $\mathrm{Fe}^{3+}$ site. This may be another reason for the inactivity of $\mathrm{Fe}_{3} \mathrm{O}_{4}$, since less $\mathrm{Fe}^{3+}$ species exist in this phase, when compared to $\mathrm{Fe}_{2} \mathrm{O}_{3}$. At atomic defect sites on the $\alpha-\mathrm{Fe}_{2} \mathrm{O}_{3}(0001)$ surface, a more favorable geometric arrangement of the basic and acidic sites might be established than on regular surface areas, which is important in order to allow their effective action in an almost simultaneous manner. The NEXAFS measurements have shown that both EB and St adsorb with the 
phenyl group lying almost flat on the surface, most likely due to interaction of the $\pi$ system with acidic Fe cations. Since defects do not introduce chemisorption sites, the model presented in Fig. 7.6 was proposed. The active site is composed of regular acidic terrace site and a defect next to it, which exposes basic $\mathrm{O}$ sites in a favourable geometric arrangement. The terrace site is responsible for adsorbing and holding the molecule on site (step 1) long enough to enable the dehydrogenation reaction (step 2). After desorption of the product molecule St (step 3), the hydrogen has to be removed. Nothing is known so far about the mechanism of this step 4. Direct desorption of $\mathrm{H}_{2}$ seems unlikely, since it must be bound similarly strong as in the ethyl group of EB from which it was removed. It is possible that it desorbs in form of water consuming a lattice $\mathrm{O}$ atom. This may be replaced by dissociation of a water moleculs from the feed, which may occur locally and temporally decoupled from the EB dehydrogenation (Mars-van-Krevelen mechanism).

TABLE 7.1. Energies $E_{\text {des }}$ and frequency factors $v_{\text {des }}$ for desorption of EB and St from $\mathrm{Fe}_{3} \mathrm{O}_{4}(111)$ and $\alpha-\mathrm{Fe}_{2} \mathrm{O}_{3}(0001)$ model catalyst surfaces (from Table 6.4) as well as total coverage $\Theta=\Theta_{E B}+\Theta_{S t}$ and coverage ratio $\Theta_{E B} / \Theta_{S t}$ extrapolated from these data for conditions of technical styrene synthesis ( $T=900 \mathrm{~K}, p=200 \mathrm{mbar}$ ) using Langmuir formalism for competitive adsorption. Sticking coefficients were assumed to be $s=1$. For details see [363].

\begin{tabular}{|c|cc|cc|}
\hline & \multicolumn{2}{|c|}{$\mathrm{Fe}_{3} \mathrm{O}_{4}(111)$} & \multicolumn{2}{c|}{$\alpha-\mathrm{Fe}_{2} \mathrm{O}_{3}(0001)$} \\
& $\mathrm{EB}$ & $\mathrm{St}$ & $\mathrm{EB}$ & $\mathrm{St}$ \\
\hline$E_{\text {des }}[\mathrm{kJ} / \mathrm{mol}]$ & 86 & 118 & 64 & 73 \\
$v_{\text {des }}\left[\mathrm{s}^{-1}\right]$ & $1 \times 10^{12}$ & $3 \times 10^{11}$ & $1 \times 10^{12}$ & $5 \times 10^{12}$ \\
\hline coverage $\Theta$ & \multicolumn{2}{|c|}{1} & \multicolumn{2}{c|}{0.35} \\
$\Theta_{E B} / \Theta_{S t}$ & \multicolumn{2}{|c|}{$<0.01$} & \multicolumn{2}{|c|}{$\approx 1.3$} \\
\hline
\end{tabular}
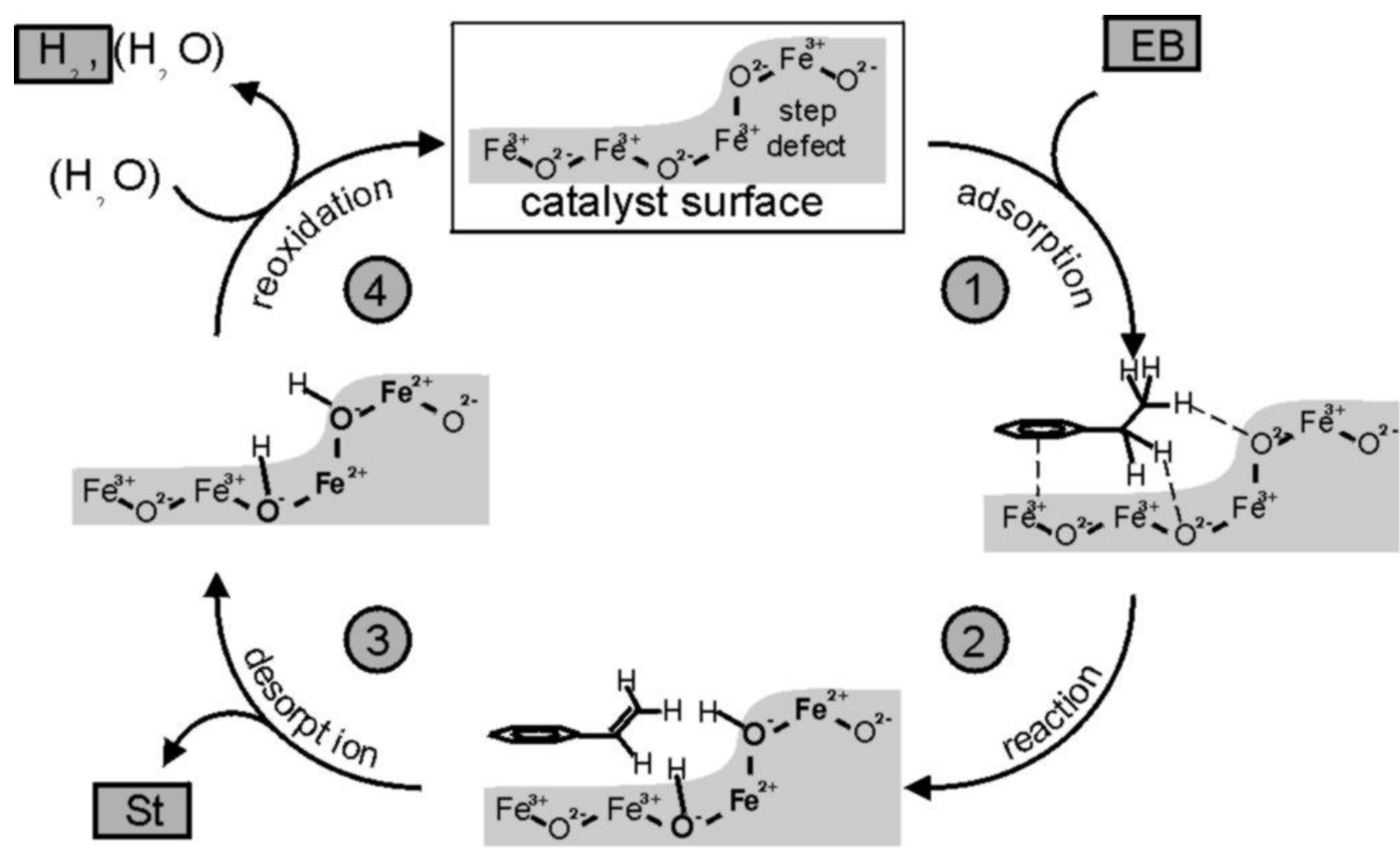

Fig. 7.6. Reaction mechanism proposed for dehydrogenation of $\mathrm{EB}$ on defective $\alpha-\mathrm{Fe}_{2} \mathrm{O}_{3}(0001)$ model catalysts. After [365]. 
Using PEEM, the formation of carbonaceous surface deposits on the model catalyst surfaces was observed. The pattern formation shows that this is a site-selective process. It presumably starts at mesoscopic structural defects, which might have different properties than the atomic defect sites for the styrene formation, and continues to grow from there forming patches on the catalyst surfaces. Such extended defect sites exist both on the active $\mathrm{Fe}_{2} \mathrm{O}_{3}$ and on the inactive $\mathrm{Fe}_{3} \mathrm{O}_{4}$ films. The growth of the carbonaceous deposits is clearly correlated to the film activities. With increasing catalytic activity its formation is enhanced, and after prolonged batch-reactor operation the active $\mathrm{Fe}_{2} \mathrm{O}_{3}$ films are completely overgrown with deposits. At this point, they are deactivated, which may be caused by the hydrocarbon deposits, which here turn out not to be catalytically active. However, a partial reduction of $\mathrm{Fe}_{2} \mathrm{O}_{3}$ to $\mathrm{Fe}_{3} \mathrm{O}_{4}$ during the reaction cannot be ruled out.

The correlation between the catalytic $\mathrm{Fe}_{2} \mathrm{O}_{3}$ film activity and the formation of carbonaceous deposits indicates the possibility that they act as catalysts in a second reaction channel for the styrene synthesis. As discussed in Section 4, the oxy-dehydrogenation of EB to styrene over various oxide compounds and phosphates was found to be catalyzed by "active coke" that formed on these surfaces. Such a mechanism was never cosidered for Fe-oxide catalysts, as there seems to exist no evidence for it. Since the dehydrogenation of EB over Fe-oxide catalysts is performed without $\mathrm{O}_{2}$ in the feed, such a mechanism would require the dissociation of water in the first step, in order to provide the oxygen for the active coke formation. A dissociative adsorption of water was found on the Fe-oxide multilayer films, and the role of the carbonaceous surface deposits has to be investigated further in future studies.

\section{Conclusion}

A comprehensive and integrative study on well-defined single-crystalline metal-oxide model systems was presented, which combines surface-science investigations in UHV with in-situ reaction experiments at elevated gas pressures. The dehydrogenation of ethylbenzene to styrene, an important synthesis reaction in chemical industry, was chosen as model system. The goal was to clarify the reaction mechanism on an atomic scale and to learn about the surface chemistry of metal-oxides in general.

Suitable samples for this project became available by the heteroepitaxial growth of well-ordered Feoxide thin films onto Pt single-crystal substrates. Three different oxide phases could be prepared, which allowed to study the influence of the oxide composition and surface structure on the chemical reactivity. Ultrathin $\mathrm{FeO}(111)$ films 1-2 monolayer thick are strongly relaxed and form a purely $\mathrm{O}$ terminated surface. They are not equivalent to the corresponding bulk oxide, but they serve as a valuable model system for studying the influence of the surface termination on the adsorption behavior of metal-oxides. $\mathrm{Fe}_{3} \mathrm{O}_{4}(111)$ and $\alpha-\mathrm{Fe}_{2} \mathrm{O}_{3}(0001)$ multilayer films at least $10 \mathrm{~nm}$ thick turned out to be equivalent to the corresponding bulk oxides, both with respect to their geometric and to their electronic structure. Therefore, these multilayer films represent model systems that simulate Fe-oxide catalysts in a realistic way. Both oxides form unreconstructed bulk terminated surface structures with strong interlayer relaxations down to the fourth layer. The $\mathrm{Fe}_{3} \mathrm{O}_{4}(111)$ exposes $\mathrm{Fe}$ cations in the topmost layer with a hexagonal close-packed $\mathrm{O}$ layers underneath. Although the $\alpha-\mathrm{Fe}_{2} \mathrm{O}_{3}(0001)$ surface most likely is $\mathrm{O}$ terminated, the $\mathrm{Fe}$ cations of the second layer are very near to the surface due, to the strong relaxation of the first interlayer distance, and act as acidic sites as well. Therefore, pairs of acidic metal sites and neighboring basic $\mathrm{O}$ sites are present on these surfaces. It is very likely that these surface structures also exist under the conditions of the technical styrene synthesis reaction, because temperature and $\mathrm{O}_{2}$ partial pressure created by homolytic dissociation of steam in the reactor atmosphere are very similar to the preparation conditions applied here. The mesoscopic film morphologies and atomic surface defect concentrations were varied within certain ranges, and the 
nature of the dominating type of atomic surface defects was clarified. This allowed to systematically study the role of surface structure, stoichiometry and surface defects for the Fe-oxide surface chemistry.

The adsorption studies show that the chemical reactivity of the Fe-oxide surfaces is related to the presence of Fe cations. Whereas the purely O-terminated $\mathrm{FeO}(111)$ surface is completely inert towards chemisorption of all gases investigated here, molecular chemisorption of etlylbenzene and styrene is observed on regular and defect-free surface areas of $\mathrm{Fe}_{3} \mathrm{O}_{4}(111)$ and $\mathrm{Fe}_{2} \mathrm{O}_{3}(0001)$, where Fe cations act as acidic adsorption sites. For low coverage, both $\mathrm{EB}$ and $\mathrm{St}$ are oriented with the phenyl ring almost parallel to the surface. On $\mathrm{Fe}_{3} \mathrm{O}_{4}(111)$, water is even chemisorbed dissociatively, due to the presence of reactive acidic $\mathrm{Fe}$ cations and basic $\mathrm{O}$ neighbors. Because these sites are arranged geometrically in a favourable way, they can act in a cooperative way. The water molecule is first adsorbed via the $\mathrm{O}$ atom onto the $\mathrm{Fe}$ site and then dissociates into $\mathrm{OH}$ and $\mathrm{H}$ species. The $\mathrm{OH}$ group remains on the $\mathrm{Fe}$ site and the $\mathrm{H}$ atom is adsorbed on an $\mathrm{O}$ site. That the chemisorption reactivity is caused by the topmost $\mathrm{Fe}$ cations was further substantiated by the decrease of chemisorbed species, when the number of point defects was increased. These point defects were identified as Fe cation vacancies. No other experiments on purely $\mathrm{O}$ terminated metal-oxide surfaces have been performed so far, but these findings are in line with results obtained on other polar and non-polar oxide surfaces [10]. Non-polar surfaces were found to be less reactive towards chemisorption, because the metal cations are shielded by the large $\mathrm{O}$ ions at the surface, wheras on polar surfaces with more exposed metal cations the reactants can approach these sites more closely, which leads to stronger chemisorption. It may be postulated as a general rule that chemisorption reactivity of metal-oxides requires the presence of acidic metal sites at the surface. For the next step in a chemical reaction, such as dissociation or dehydrogenation, the cooperative action of acidic and basic sites is required as well. The geometric arrangement of these pairs of sites on the surface and their strength of acidity and basicity most likely determines, which particular surface reaction becomes possible. Since this also depends on the geometric dimensions of the involved molecules, selectivity is also determined by these geometric factors. The adsorption studies showed that the dissociative adsorption of water is possible on the regular surfaces, but dehydrogenation of ethylbenzene to styrene does not occur.

The intrinsic kinetic parameters for the adsorption-desorption processes of the molecules involved in the styrene synthesis reaction were determined quantitatively by the UHV experiments on the model catalyst surfaces. For the educt and product molecules ethylbenzene and styrene non-activated adsorption via weakly bound mobile precursor states into chemisorbed and physisorbed adsorption states were found, with high sticking coefficients almost upto the corresponding saturation coverages. Using the Langmuir isotherm formalism for competitive adsorption, the relative coverages of the adsorption states were extrapolated to the technical styrene synthesis reaction conditions. It turned out that the $\mathrm{Fe}_{3} \mathrm{O}_{4}$ surface should be blocked completely by the styrene product molecules, which would explain their inactivity. In contrast, the $\mathrm{Fe}_{2} \mathrm{O}_{3}$ surface is only partially covered by roughly equal amounts of EB and St and site blocking should not occur.

The batch-reactor experiments performed at elevated gas pressures were in agreement with these extrapolation results. $\mathrm{Fe}_{2} \mathrm{O}_{3}$ is the catalytically active Fe-oxide phase for the dehydrogenation of ethylbenzene to styrene, whereas no styrene formation was observed on the $\mathrm{Fe}_{3} \mathrm{O}_{4}$ films. However, it turned out that the dehydrogenation does not take place on regular surface areas of $\mathrm{Fe}_{2} \mathrm{O}_{3}$, but needs atomic surface defects. Since no chemisorption states related to surface defects could be observed, this led to a model with the active site consisting of a regular chemisorption site and of a defect site next to it. No details about the structure of the defect sites are known so far, but it is likely that they expose basic $\mathrm{O}$ sites capable to dehydrogenate the ethyl group of EB. It can be anticipated that the precise geometry of this composite active site is not only necessary for its activity, but also for the selectivity of the reaction. 
A second reaction channel involving catalytically active carbonaceous deposits might be possible. This is indicated by the correlation between the catalytic $\mathrm{Fe}_{2} \mathrm{O}_{3}$ film activity and the formation of carbonaceous deposits as observed by photoelectron emission microscopy. The deposits presumably start to form at mesoscopic structural defects that are different from the atomic defect sites active for the styrene formation. After a prolonged batch-reactor operation the active $\mathrm{Fe}_{2} \mathrm{O}_{3}$ films are completely overgrown and the films are deactivated. This may be caused both by the deposits and by a partial reduction to $\mathrm{Fe}_{3} \mathrm{O}_{4}$ during the reaction. Carbon based catalysts for an oxo-dehydrogenation must contain oxygen species. Since no $\mathrm{O}_{2}$ is leaked into the batch reactor, it must be provided by reduction of the Fe-oxide or the water. The latter mechanism would require the dissociative adsorption of water in the first place, which indeed was found on the Fe-oxide model systems. Therefore the role of the carbonaceous surface deposits has to be investigated further.

The most active catalysts are potassium promoted $\mathrm{Fe}$-oxides. It has been proposed that the active sites on unpromoted and promoted catalysts might be the same, since the apparent activation energy for the overall reaction is similar [194]. However, as long as the mechanism on a molecular level is not known for both catalysts, this remains speculation. First steps in the preparation and characterization of promoted model catalyst films have been done, which indicate that the mechanisms on promoted and unpromoted catalysts are quite different $[363,365]$. Since knowledge of promoted model catalysts is much less, this review was confined to unpromoted films.

\section{Acknowledgement}

Most of the work presented in this review was performed in the Department of Inorganic Chemistry, Fritz Haber Institute of the Max Planck Gesellschaft in Berlin, Germany and we gratefully acknowledge countless fruitful discussions with its head Robert Schlögl and his permanent support. The major contributions were done by the following members, former members or guests of the model catalysis group: Michael Ritter, Detlef Zscherpel, Yvonne Joseph, Shamil Shaikhutdinov, Christian Kuhrs, Guido Ketteler, Dietrich Frickel, Sebastien Ragot, Runsheng Zhai, Andreas Munz, Yoshinobu Arita and our ingenieur Manfred Swoboda. We are indebted to them for their excellent contributions. We further acknowledge fruitful collaborations with Thomas Schedel-Niedrig, Axel Knop-Gericke, Michael Hävecker, Yong Cai, Alexander Bradshaw, Xiao-Gang Wang, Matthias Scheffler, Vladimir

Roddatis and Dangsheng Su from the Fritz Haber Institute, with $\mathrm{Ch}$. Wöll and M. Wühn from the Ruhr Universität Bochum, Lehrstuhl für Physikalische Chemie I, and with the BESTEC company.

\section{References}

[1] G. Ertl, H. Knözinger and J. Weitkamp (Eds.); Handbook of Heterogeneous Catalysis, WileyVCH, Weinheim (1997).

[2] H.-P. Boehm and H. Knözinger, in Catalysis Science and Technology, J.R. Anderson and M. Boudart (Eds.), Springer-Verlag Berlin (1983), Vol. 4, p. 39.

[3] H.H. Kung, Transition Metal-oxides: Surface Chemistry and Catalysis, Studies in Surface Science and Catalysis 45, Elsevier Science, New York (1991).

[4] H.-J. Freund and E. Umbach, Adsorption on Ordered Surfaces of Ionic Solids and Thin Films, Vol. 33 of Springer Series in Surface Science, Springer, Berlin (1993).

[5] V.E. Henrich and P.A. Cox, The Surface Science of Metal-oxides, Cambridge University Press, Cambridge (1994).

[6] C. Noguera, Physics and Chemistry at Oxide Surfaces, Cambridge University Press, Cambridge (1996).

- 121 -"2kyEi9FYSQjBVrZxIPQ.FHIAC_WRa02_review.doc", Datum: 19.02.03 
[7] G. Centi, in: Catalysis from A to Z, B. Cornils, W.A. Herrmann, R. Schlögl and C.-H. Wong (Eds.), Wiley-VCH, Weinheim (2000).

[8] P.W. Jacobs and G.A. Somorjai, J. Mol. Catal. A 131, 5 (1998).

[9] R. Schlögl, in Handbook of Heterogeneous Catalysis, G. Ertl, H. Knözinger, J. Weitkamp, (Eds.), Wiley-VCH, Weinheim (1997), Vol. 4, p. 1697.

[10] H.-J. Freund, Angewandte Chemie 109/5, 444 (1997).

[11] C. Noguera, J. Phys. Cond. Matter 12, R367 (2000).

[12] K. Kochloefl, in Handbook of Heterogeneous Catalysis, G. Ertl, H. Knözinger, J. Weitkamp, (Eds.), Wiley-VCH (1997), Vol. 5, p. 2151.

[13] I. Langmuir, J. Amer. Chem. Soc. 38, 2221 (1916).

[14] I. Langmuir, J. Amer. Chem. Soc. 40, 1361 (1918).

[15] G. Wedler, Adsorption, Chemische Taschenbücher, Vol. 9, Verlag Chemie, Weinheim (1970).

[16] K. Christmann, Surface Physical Chemistry, Steinkopff, Darmstadt (1991).

[17] A. Clark, The Theory of Adsorption and Catalysis, Academic Press, New York (1970).

[18] J.G. Dash, Films on Solid Surfaces, Academic Press, New York (1975).

[19] W.A. Steele, The Interaction of Gases with Solid Surfaces, Pergamon Press, Oxford (1974).

[20] H.-J. Freund, in Handbook of Heterogeneous Catalysis, G. Ertl, H. Knözinger, J. Weitkamp, (Eds.), Wiley-VCH (1997), Vol. 4, p. 911.

[21] J.E. Lennard-Jones, Trans. Faraday Soc. 28, 333 (1932).

[22] V. Bortolani, N.H. March, and M.P. Tosi, (Eds.), Interaction of Atoms and Molecules with Solid Surfaces, Plenum Press, New York (1990).

[23] P.S. Bagus and G. Pacchioni, J. Electr. Spectr. Rel. Phen. 69, 109 (1994).

[24] F. Boszo, G. Ertl, M. Grunze, and M. Weiss, J. Catal. 49, 18 (1977).

[25] G. Ertl, in Catalytic Ammonia Synthesis, J.R. Jennings, (Ed.), Plenum, New York (1983).

[26] D. Tomanek and K.H. Bennemann, Phys. Rev. B31, 2488 (1984).

[27] D.A. King and D.P. Woodruff, (Eds), The Chemical Physics of Solid Surfaces and Heterogeneous Catalysis, Elsevier, Amsterdam (1990), Vol. 3.

[28] K. Christmann, Surf. Sci. Rep. 9, 1 (1988).

[29] K. Wandelt, Surf. Sci. Rep. 2, 1 (1982).

[30] C.R. Brundle and J.Q. Broughton, in The Chemical Physics of Solid Surfaces and Heterogeneous Catalysis, D.A. King and D.P. Woodruff, (Eds.), Elsevier, Amsterdam (1990), Vol. 3.

[31] G. Blyholder, J. Phys. Chem. 68, 2772 (1964).

[32] B. Hammer, Y. Morikawa, and J.K. Norskov, Phys. Rev. Lett. 76, 2141 (1996).

[33] J.K. Norskov and P. Stoltze, Surf. Sci. 189, 91 (1987).

[34] A. Ruban, B. Hammer, P. Stoltze, H.L. Skriver and J.K. Norskov, J. Mol. Catal. A 115, 421 (1997).

[35] R.J. Behm, Acta Phys. Pol. A 93, 259 (1998).

[36] B. Gleich, M. Ruff, and R.J. Behm, Surf. Sci. 386, 48 (1997).

[37] M. Ruff, S. Frey, B. Gleich, and R.J. Behm, Appl. Phys. A 66, S513 (1998).

[38] M. Pöhlchen and V. Staemmler, J. Chem. Phys. 97, 2583 (1992).

[39] V. Staemmler, in Adsorption on Ordered Surfaces of Ionic Solids and Thin Films, H.-J. Freund, E. Umbach, (Eds.), Springer Series in Surface Science Vol. 33, Springer, Heidelberg (1993), p. 169.

[40] G. Pacchioni, G. Cogliandro, and P.S. Bagus, Surf. Sci. 225, 344 (1991).

[41] G. Pacchioni, T. Minerva, and P.S. Bagus, Surf. Sci. 275, 450 (1992).

[42] P. Pacchioni, Surf. Sci. 281, 257 (1993).

[43] G. Pacchioni and P.S. Bagus, in Adsorption on Ordered Surfaces of Ionic Solids and Thin Films, H.-J. Freund, E. Umbach, (Eds.), Springer Series in Surface Sciences Vol. 33, Springer, Heidelberg (1993), p. 180. 
[44] K.M. Neymann and N. Rösch, J. Chem. Phys. 168, 267 (1993).

[45] K.M. Neymann and N. Rösch, Surf. Sci. 297, 223 (1993); Chem. Phys. 177, 561 (1993).

[46] C. Pisani, R. Dovesi, R. Nada, and S. Tamiro, Surf. Sci. 216, 267 (1989).

[47] M.A. Nygren and L.G.M. Petterson, J. Electr. Spectr. Rel. Phen. 69, 43 (1994).

[48] S.M. Vesecky, X. Xu, D.W. Goodman, J. Vac. Sci. Technol. A 12, 2114 (1994).

[49] J. Heidberg and D. Meine, Ber. Bunsenges. Phys. Chem. 97, 211 (1993).

[50] D. Cappus, J. Klinkmann, H. Kuhlenbeck, and H.-J. Freund, Surf. Sci. L421, 325 (1995).

[51] R. Gerlach, A. Glebov, G. Lange, J.P. Toennies, and H. Weiss, Surf. Sci. 331, 2490 (1995).

[52] C. Xu, B. Dillmann, H. Kuhlenbeck, and H.-J. Freund, Phys. Rev. Lett. 67, 3551 (1991).

[53] M.C. Wu, C.M. Truong, and D.W. Goodman, J. Phys. Chem. 97, 4182 (1993).

[54] J. Heidberg, L. Cabigon, E. Kampshoff, M. Kandel, R. Kühnemuth, D. Meine, B. Redlich, O. Schönekäs, M. Suhren, H. Weiss, and D. Wetter, in Adsorption on Ordered Surfaces of Ionic Solids and Thin Films, H.-J. Freund, E. Umbach, (Eds), Springer Series in Surface Sciences Vol. 33, Springer, Heidelberg (1993), p. 46.

[55] R.G. Pearson, Hard and Soft Acids and Bases, Dowden, Hutchinson, and Ross Publ., Stroudsburg (1973).

[56] M.C. Kung and H.H. Kung, Catal. Rev. Sci. Eng. 27, 425 (1985).

[57] H. Knözinger, Adv. Catal. 25, 184 (1976).

[58] P.A. Thiel and T.E. Madey, Surf. Sci. Rep. 7, 211 (1987).

[59] P. Liu, T. Kendelewicz, G.E. Brown, Jr., G.A. Parks and P. Pianetta, Surf. Sci. 416, 326 (1998).

[60] M.A. Henderson, Surf. Sci. 319, 315 (1994).

[61] M.A. Henderson, S.A. Joyce and J.R. Rustad, Surf. Sci. 417, 66 (1998).

[62] P. Liu, T. Kendelewicz, G.E. Brown, Jr. and G.A. Parks, Surf. Sci. 412/413, 287 (1998).

[63] P. Liu, T. Kendelewicz, G.E. Brown, E.J. Nelson and S.A. Chambers, Surf. Sci. 417, 53 (1998).

[64] D. Cappus, C. Xu, D. Ehrlich, B. Dillmann, C.A. Ventrice, K. Al Shamery, H. Kuhlenbeck, and H.-J. Freund, Chem. Phys. 177, 533 (1993).

[65] F. Rohr, K. Wirth, J. Libuda, D. Cappus, M. Bäumer, and H.-J. Freund, Surf. Sci. L977, 315 (1994).

[66] D. Cappus, M. Haßel, E. Neuhaus, M. Heber, F. Rohr, and H.-J. Freund, Surf. Sci. 337, 268 (1995).

[67] P. Mars and D. van Krevelen, Chem. Eng. Sci. Suppl. 3, 41 (1954).

[68] D. Cappus, M. Haßel, E. Neuhaus, M. Heber, F. Rohr and H.-J. Freund, Surf. Sci. 337, 268 (1995).

[69] W. Ranke and W. Weiss, Surf. Sci. 465, 317 (2000).

[70] K. Christmann and J.E. Demuth, Surf. Sci. 120, 291 (1982).

[71] W. Ranke and W. Weiss, Surf. Sci. Surf. Sci. 414, 236 (1998).

[72] C.E. Borroni-Bird, N. Al-Sarraf, S. Andersson, and D.A. King, Chem. Phys. Lett. 183, 516 (1991).

[73] C.E. Borroni-Bird and D.E. King, Rev. Sci. Inst. 62, 1 (1991).

[74] G. Ehrlich, Adv. Catal. 14, 255 (1963).

[75] P.A. Redhead, Vacuum 12, 203 (1962).

[76] D.A. King, Surf. Sci. 47, 384 (1975).

[77] D. Menzel, in Interactions on Metal Surfaces, Topics in Applied Physics Vol. 4, Springer, Heidelberg (1975).

[78] L.A. Petermann, Prog. Surf. Sci. 1, 2 (1972).

[79] M. Polanyi, Trans. Faraday Soc. 28, 314 (1932).

[80] J.W. Niemantsverdriet, Spectroscopy in Catalysis: An Introduction, Wiley-VCH, Weinheim (2000).

[81] M.J. Dresser, T.E. Madey, and J.T. Yates, Surf. Sci. 42, 533 (1974).

[82] E. Habenschaden and J. Küppers, Surf. Sci. 138, L147 (1984). 
[83] H.J. Kreuzer and S.H. Payne, Astek, Helix Science Applications, Armandale (1993).

[84] D. Zscherpel, W. Weiss and R. Schlögl, Surf. Sci. 382, 326 (1997).

[85] C.M. Chan and A.R. Weinberg, Surf. Sci. 1, 360 (1978).

[86] J.L. Falconer and R.J. Madix, Surf. Sci. 48, 393 (1975).

[87] P.W. Atkins, Physical Chemistry, Oxford University Press, (1987).

[88] Y. Joseph, W. Ranke and W. Weiss, J. Phys. Chem. B 104, 3224 (2000).

[89] M.J. Temkin and V. Pyshev, Acta physicochim. USSR 12, 327 (1940).

[90] S. Brunauer, P.H. Emmett, and E. Teller, J. Amer. Chem. Soc. 60, 309 (1938).

[91] H. Freundlich, Colloid and Capillary Chemistry, Methew, London (1926).

[92] J. Zeldowitsch, Acta physicochim. USSR 1, 961 (1934).

[93] B. Poelsema, L.K. Verheij, and G. Comsa, Surf. Sci. 152/153, 496 (1985).

[94] K.D. Rendulic, Appl. Phys. A 47, 55 (1988).

[95] J.N. Russel Jr., I. Chorkendorff, A.M. Lanzilotto, M.D. Alvey, and J.T. Yates Jr., J. Chem. Phys. 85, 6186 (1986).

[96] R. Raval, M.A. Harrison, and D.A. King, in Chemisorption Systems, The Chemical Physics of Solid Surfaces and Heterogeneous Catalysis, D.A. King and D.P. Woodruff, (Eds.), Part B., Vol. 3, Elsevier, New York (1990).

[97] H.A. Michelsen, C.T. Rettner, and D.J. Auerbach, in Surface Reactions, R.J. Madix (Ed.), Springer Series in Surface Science Vol. 34, Springer, Heidelberg (1994), p. 185.

[98] D.A. King and M.G. Wells, Surf. Sci. 29, 454 (1972).

[99] D.A. King and M.G. Wells, Proc. Roy. Soc. London Ser. A. 339, 245 (1974).

[100] K.D. Rendulic, G. Anger, A. Winkler, Surf. Sci. 208, 404 (1989).

[101] C.T. Rettner, L.A. DeLonise, and D.J. Auerbach, J. Phys. 85, 1131 (1986).

[102] A.K. Hamza, H.-P. Steinrück, R.J. Madix, J. Chem. Phys. 86, 6506 (1987).

[103] C.T. Rettner, L.A. DeLonise, and D.J. Auerbach, J. Phys. 85, 1131 (1986).

[104] M.B. Lee, Q.Y. Yang, and S.T. Ceyer, J. Chem. Phys. 86, 2724 (1987).

[105] M.A. Morris, M. Bowker, and D.A. King, in Simple Processes at the Gas Solid Interface, C.H. Bamford, C.F.H. Tipper, R.G. Compton, (Eds.), Elsevier, Amsterdam (1984), p. 1.

[106] P.J. Kisliuk, J. Phys. Chem. Sol. 5, 78 (1958).

[107] P.J. Kisliuk, J. Phys. Chem. Sol. 3, 95 (1957).

[108] K. Christmann, O. Schober, G. Ertl, and M. Neumann, J. Chem. Phys. 60, 4528 (1974).

[109] T. Engel, J. Chem. Phys. 69, 373 (1978).

[110] G. Ertl and J. Koch, Z. Naturforsch. 25a, 1906 (1970).

[111] C.T. Campbell, G. Ertl, H. Kuipers, and J. Segner, Surf. Sci. 107, 207 (1981).

[112] J. K. Norskov and P. Stoltze, in Handbook of Heterogeneous Catalysis, G. Ertl, H. Knözinger, J. Weitkamp, (Eds.), Wiley-VCH, Weinheim (1997), Vol. 3, p. 983.

[113] B. Hammer, M. Scheffer, K.W. Jacobsen, and J.K. Norskov, Phys. Rev. Lett. 73, 1400 (1994).

[114] J.W. Evans, J. Chem. Phys. 97, 572 (1992).

[115] M.W. Deem, W.H. Weinberg, and H.C. Kang, Surf. Sci. 276, 99 (1992).

[116] V.P. Zhdanov and B. Kasemo, Surf. Sci. Rep. 20, 111 (1994).

[117] G. Ertl, in Catalytic Ammonia Synthesis, J.R. Jennings (Ed.), Plenum, New York (1993).

[118] G. Ertl, Surf. Sci. 287, 1 (1993).

[119] R.I. Masel, Adsorption and Reaction on Solid Surfaces, Wiley, New York (1996).

[120] J. Rostrup-Nielsen, in Elementary Reaction Steps in Heterogeneous Catalysis, Kluwer Academic Publishers (1993), p. 441.

[121] O. Hinrichsen, F. Rosowski, M. Muhler and G. Ertl, Chem. Eng. Sci. 51, 1683, (1996).

[122] P.B. Rasmussen, P.M. Holmblad, T. Askgaard, C.V. Ovesen, P. Stoltze, J.K. Norskov, and I. Chorkendorff, Catal. Lett. 26, 373 (1994).

[123] J.E. Rekoske, R.D. Cartright, S.A. Goddard, S.B. Sharma, and J.A. Dumesic, J. Phys. Chem. 96, 1880 (1992). 
[124] S.H. Oh, G.B. Fisher, J.E. Carpenter, D.W. Goodman, J. Catal. 100, 360 (1986).

[125] P. Stoltze and J.K. Norskov, Phys. Rev. Lett. 55, 2502 (1985).

[126] R. Schlögl, R.C. Schoonmaker, M. Muhler and G. Ertl, Cat. Lett. 1, 237 (1988).

[127] M. Muhler, F. Rosowski and G. Ertl, Cat. Lett. 24, 317 (1994).

[128] K. Heinz, Progr. Surf. Sci. 27, 239 (1988), Vacuum 41, 328 (1990).

[129] G.A. Somorjai, Introduction to Surface Chemistry and Catalysis, Wiley, New York (1994).

[130] A. Knop-Gericke, M. Hävecker, T. Schedel-Niedrig, and R. Schlögl, Ang. Chem. 13, 110 (1998).

[131] X. Bao, M. Muhler, Th. Schedel-Niedrig, and R. Schlögl, Phys. Rev. B 54, 2249 (1996).

[132] D.W. Goodman, R.D. Kelly, T.E. Madey, J.T. Yates, J. Catal. 63, 226 (1980).

[133] R.D. Kelley and D.W. Goodman, Surf. Sci. 123, L743 (1982).

[134] C.M. Eggleston and M.F. Hochella Jr., Am. Mineral. 77, 911 (1992).

[135] R.L. Kurtz and V.E. Henrich, Surf. Sci. 129, 345 (1983).

[136] R.L. Kurtz and V.E. Henrich, Phys. Rev. B 36, 3413 (1987).

[137] A.R. Lennie, N.G. Condon, F.M. Leibsle, P.W. Murray, G. Thornton and D.J. Vaughan, Phys. Rev. B 15, 10244 (1996).

[138] N.G. Condon, F.M. Leibsle, A.R. Lennie, P.W. Murray, D.J. Vaughan and G. Thornton, Phys. Rev. Lett. 75, 1961 (1995).

[139] R. Jansen, V.A.M. Brabers and H. van Kempen, Surf. Sci. 328, 237 (1995).

[140] Q. Guo and P.J. Moller, Surf. Sci. 340, L999 (1995).

[141] G. Tarrach, D. Bürgler, T. Schaub, R. Wiesendanger and H.-J. Güntherodt, Surf. Sci. 285, 1 (1993).

[142] A. Stierle, P. Bödecker and H. Zabel, Surf. Sci. 327, 9 (1995).

[143] R.M. Jaeger, H. Kuhlenbeck, H.-J. Freund, M. Wuttig, W. Hoffmann, R. Franchy and H. Ibach, Surf. Sci. 259, 235 (1991).

[144] D.R. Mueller, R.L. Kurtz, R.L. Stockbauer and T.E. Madey, Surf. Sci. 237, 72 (1990).

[145] T. Inoue, Y. Yamamoto, M. Satoh, A. Ide and S. Katsumata, Thin Solid Films 281, 24 (1996).

[146] K.-D. Schierbaum, Surf. Sci. 399, 29 (1998).

[147] V. Maurice, M. Salmeron and G.A. Somorjai, Surf. Sci. 237, 116 (1990).

[148] Th. Bertrams and H. Neddermeyer, J. Vac. Sci. Technol. B 14, 1141 (1996).

[149] W.S. Oh, C. Xu, D.Y. Kim, D.W. Goodman, J. Vac. Sci. Technol. A 15, 1710 (1997).

[150] L. Zhang, M. Kuhn and U. Diebold, Surf. Sci. 375, 1 (1997).

[151] K. Kishi, Y. Hayakawa, and K. Fujiwara, Surf. Sci. 356, 171 (1996).

[152] P.J. Chen and D.W. Goodman, Surf. Sci. 312, L767 (1994).

[153] S.C. Street, C. Xu and D.W. Goodman, Ann. Rev. Phys. Chem. 48, 43 (1997).

[154] S.A. Chambers, Surf. Sci. Reports 39, 105 (2000).

[155] A.B. Boffa, H.C. Galloway, P.W. Jacobs, J.J. Benitez, J.D. Batteas, M. Salmeron, A.T. Bell and G.A. Somorjai, Surf. Sci. 326, 80 (1995).

[156] G.S. Hermann, M.C. Gallagher, S.A. Joyce, and C.H.F. Peden, J. Mater. Res. B 14, 1126 (1996).

[157] C. Hardacre, G.M. Roe, and R.M. Lambert, Surf. Sci. 326, 1 (1995).

[158] G.H. Vurens, M. Salmeron and G.A. Somorjai, Surf. Sci. 201, 129 (1988).

[159] G.H. Vurens, D.R. Strongin, M. Salmeron and G.A. Somorjai, Surf. Sci. 199, L387 (1988).

[160] G.H. Vurens, V. Maurice, M. Salmeron and G.A. Somorjai, Surf. Sci. 268, 170 (1992).

[161] H.C. Galloway, J.J. Benitez and M. Salmeron, Surf. Sci. 298, 127 (1993).

[162] H.C. Galloway, J.J. Benítez and M. Salmeron, J. Vac. Sci. Technol. A 12, 2302 (1994).

[163] H.C. Galloway, P. Sautet and M. Salmeron, Phys. Rev. B 54, R11145 (1996).

[164] Y.J. Kim, C. Westphal, R.X. Ynzunza, H.C. Galloway, M. Salmeron, M.A. Van Hove and C.S. Fadley, Phys. Rev. B 55, R13448 (1997). 
[165] J.M. Gaines, P.J.H. Bloemen, J.T. Kohlhepp, C.W.T. Bulle-Lieuwma, R.M. Wolf, A. Reinders, R.M. Jungblut, P.A.A. van der Heijden, J.T.W.M. van Eemeren, J. Van de Stegge, W.J.M. de Jonge, Surf. Sci. 373, 85 (1997).

[166] T. Fujii, M. Takano, R. Katano, Y. Bando and Y Isozumi, J. Appl. Phys. 666, 3168 (1989).

[167] F.C. Voogt, T. Hibma, G.L. Zhang, M. Hoefman and L. Niesen, Surf. Sci. 331, 1508 (1995).

[168] Y. Gao, Y.J. Kim and S.A. Chambers, J. Vac. Sci. Technol. A 15, 332 (1997).

[169] Y. Gao, Y.K. Kim, S. Thevuthasan, and S.A. Chambers, J. Appl. Phys. 81, 3253 (1997).

[170] Y.J. Kim, Y. Gao and S.A. Chambers, Surf. Sci. 371, 358 (1997).

[171] D.M. Lind, S.D. Berry, G. Chern, H. Mathias and L.R. Testardi, Phys. Rev. B 45, 1838 (1992).

[172] P.J. van der Zaag, A.R. Ball, L.F. Feiner, and R.M. Wolf, J. Appl. Phys. 79, 5103 (1996).

[173] A.M. Stoneham, in Defects and their Structure in Non-Metallic Solids, B. Henderson and A.E. Hughes, (Eds.), Plenum, New York (1976).

[174] P.W. Tasker, J. Phys. C: Solid State Phys. 12, 4977 (1979).

[175] D.M. Heyes, M. Barber, and J.H.R. Clarke, J. Chem. Soc. Faraday Trans. II 10, 1485 (1977).

[176] D.E. Parry, Surf. Sci. 49, 433 (1975).

[177] R. Lacman, Colloq. Int. CNRS 152, 195 (1965).

[178] D. Wolf, Phys. Rev. Lett. 68, 3315 (1992).

[179] W. Weiss, A. Barbieri, M.A. VanHove, and G.A. Somorjai, Phys. Rev. Lett. 71, 1848 (1993).

[180] F. Rohr, M. Bäumer, H.-J. Freund, J.A. Mejias, V. Staemmler, S. Müller, L. Hammer and K. Heinz, Surf. Sci. 372, L291 (1997); Surf. Sci. 389, 391 (1997).

[181] I. Manassidis, A. de Vita and M.J. Gillan, Surf. Sci. 285, L517 (1993).

[182] X.-G. Wang, W. Weiss, Sh.K. Shaikhutdinov, M. Ritter, M. Petersen, F. Wagner, R. Schlög1 and M. Scheffler, Phys. Rev. Lett. 81, 1038 (1998).

[183] W.S. Yang, F. Jona, and P.M. Marcus, Solid State Comm. 43, 847 (1982).

[184] R.J. Hamers, R.M. Tromp, and J.E. Demuth, Phys. Rev. B 34, 5343 (1986).

[185] A. Barbieri, W. Weiss, M.A. Van Hove, G.A. Somorjai, Surf. Sci. 302, 259 (1994).

[186] W. Weiss, M. Ritter, D. Zscherpel, M. Swoboda and R. Schlögl, J. Vac. Sci. Technol. A 16, 21 (1998).

[187] F. Nakao, Vacuum 25, 431 (1975).

[188] H. Ohlinger and S. Stadelmann, in Ullmanns Enzyklopädie der technische Chemie, Urban und Schwarzenberg, (Eds.), München-Berlin (1965), Vol. 16, p. 460.

[189] K.K. Kearby in Catalysis, P. Emmet, (Ed.), Vol.III, Rheinhold, New York (1955), p. 469.

[190] Nova Chemicals Corp., Supplemental financial and product information 2000, www.novachem.com/investorrelations/reports/2000AnnualReport/FactBook2000.pdf.

[191] E.H. Lee, Catal. Rev. 8, 285 (1973).

[192] K. Coulter, D.W. Goodman, R.G. More, Catal. Lett. 31, 1(1995).

[193] T. Hirano, Appl. Catal. 28 (1986) 119.

[194] T. Hirano, Appl. Catal. 26 (1986) 65.

[195] M. Muhler, J. Schütze, M. Wesemann, T. Rayment, A. Dent, R. Schlögl and G. Ertl, J. Catal. 126, 339 (1990).

[196] W.D. Mross, Catal. Rev. Sci. Eng. 25, 591 (1983).

[197] M Muhler, R. Schlögl and G. Ertl, J. Catal. 138, 413 (1992).

[198] S. Carra and L. Forni, Ind. Eng. Chem. Process Des. Dev. 4, 281 (1965).

[199] N.N. Lebedev, G.V. Odabashyan, V.V. Lebedev, and M.G. Markov, Kinet. Katal. 18, 1177 (1977).

[200] P. Tetenyi, Z. Paal, M. Dobrovolszk, Z. Phys. Chem. 102, 267 (1976).

[201] W.P. Addiego, C.A. Estrada, D.W. Goodman, M.P. Rosynek, R.G. Windham, J. Catal. 146, 407 (1994).

[202] M.A. Gibson and J.W. Hightower, J. Catal. 41, 420 (1976).

[203] I. Wang, J.-C. Wu and C.-S. Chung, Appl. Catal. 16, 89 (1985). 
[204] M. Muhler, PhD thesis, Fritz Haber Institute, Berlin (1989).

[205] R.H. Allen, T. Alfrey, and L.D. Yats, US Pat. 3487564 (1970).

[206] A.J. Bridgewater, R. Burch, and P.C.H. Mitchell, Appl. Catal. 4, 267 (1982).

[207] D. Richard, P. Gallezot, D. Neibecker, and I. Tkatchenko, Catal. Today 6, 171 (1989).

[208] A. Guerrero-Ruiz, A. Sepulveda-Escribano, and I. Rodriguez-Ramos, Appl. Catal. A: Gen. 81, 81 (1992).

[209] M. Ledoux and B. Djelliulli, J. Catal. 115, 580 (1989).

[210] K.M. Minachev, V.V. Kharlamov, D.B. Tagiyev, and Z.G. Zulfugarov, Zeolites, 4, 270 (1984).

[211] A. Guerrero-Ruiz and I. Rodriguez-Ramos, Carbon 32, 23 (1994).

[212] G.C. Grunewald and R.S. Drago, J. Mol. Catal. 58, 227 (1990).

[213] T. Tagawa, K. Iwayama, Y. Ishida, T. Hattori, and Y. Murakami, J. Catal. 79, 47 (1983).

[214] Y. Murakami, K. Iwayama, H. Uchida, T. Hattori, and T. Tagawa, J. Catal. 71, 257 (1981).

[215] G. Emig and H. Hofmann, J. Catal. 84, 15 (1983).

[216] A. Schraut, G. Emig, and H. Hofmann, J. Catal. 112, 221 (1988).

[217] G.E. Vrieland, J. Catal. 111 (1988) 1 and J. Catal. 111, 14 (1988).

[218] G.E. Vrieland and P.G. Menon, Appl. Catal. 77, 1 (1991).

[219] A. Schraut, G. Emig, and H.G. Sockel, Appl. Catal. 29, 311 (1987).

[220] L.E. Cadus, O.F. Gorriz, and J.B. Rivarola, Ind. Eng. Chem. Res. 29, 1143 (1990).

[221] T.G. Alkhazov and A.E. Lisovskii, Kinet. Katal. 17, 434 (1976).

[222] E. Echigoya, H. Sano, and M. Tanaka, 8th International Congress on Catalysis, Verlag Chemie, Berlin, (1992) Vol. V, p. 623.

[223] D.B. Tagiev, G.O. Gasymov, Z.G. Zulfugarov, and K.M. Minachev, 9th Actas Simp. Iberoam. Catal. 2, 944 (1984).

[224] R.M. Cornell and U. Schwertmann, The Iron-oxides, VCH Weinheim (1996).

[225] G. Ketteler, W. Weiss, W. Ranke and R. Schlögl, Phys. Chem. Chem. Phys. 3, 1114 (2001)

[226] Equitherm Version 3.0, VCH Scientific Software, VCH, Weinheim (1993).

[227] A. Muan, Am. J. Sci. 256, 171 (1958).

[228] L.S. Darken and R.W. Gurry, J. Am. Chem. Soc. 67, 1398 (1945).

[229] T. Katsura, B. Iwasaki, and S. Kimura, J. Chem. Phys. 47, 4559 (1967).

[230] R.W.G. Wyckoff, Crystal Structures, 2nd ed., Vol. I-III, Interscience Publishers (1982).

[231] E.J. Verwey, P.W. Haayman, and F.C. Romeijn, J. Chem. Phys. 15, 458 (1947).

[232] N. Tsuda, K. Nasu, A. Yanase, and K. Siratori, Electronic Conduction in Oxides, SpringerVerlag, Berlin (1991).

[233] Th. Schedel-Niedrig, W. Weiss and R. Schlögl, Phys. Rev. B 52, 17449 (1995).

[234] W. Weiss and M. Ritter, Phys. Rev. B, 59, 5201 (1999).

[235] D. Zscherpel, PhD thesis, Oberhofer, Berlin (1998).

[236] A. Yanase and K. Siratori, J. Phys. Soc. Japan 53, 312 (1984).

[237] Z. Zhang and S. Satpathy, Phys. Rev. B 44, 13319 (1991).

[238] L. Armelao, M. Bettinelli, M. Casarin, G. Granozzi, E. Tondello, J. Phys.: Cond. Matt. 7, L299 (1995).

[239] A. Fujimori, M. Saeki, N. Kimizuka, M. Taniguchi, and S. Suga, Phys. Rev. B 34, 7318 (1986).

[240] A.Fujimori, N. Kimizuka, M. Taniguchi, and S. Suga, Phys. Rev. B 36, 6691 (1987).

[241] R.J. Lad and V.E. Henrich, Phys. Rev. B 39, 13478 (1989).

[242] L.C. Davis, Phys. Rev. B 25, 2912 (1982).

[243] A. Fujimori, F. Minami, and S. Sugano, Phys. Rev. B 29, 5225 (1984).

[244] G. Van der Laan, Solid State. Comm. 42, 165 (1982).

[245] D.E. Eastman and J.L. Freeouf, Phys. Rev. Lett. 34, 395 (1975).

[246] J.J. Yeh and I. Lindau, At. Data Nucl. Tables 32, 1 (1985).

[247] Y.Q. Cai, M. Ritter, W. Weiss and A.M. Bradshaw, Phys.Rev. B 58, 5043 (1998). 
[248] F.M.F. de Groot, M. Grioni, J.C. Fuggle, J. Ghijsen, G.A. Sawatzky, and H. Petersen, Phys. Rev. B 40, 5715 (1989).

[249] C. Colliex, T. Manoubi, and C. Ortiz, Phys. Rev. B 44, 11402 (1991).

[250] J. Stöhr, NEXAFS Spectroscopy, Springer Series in Surface Science, Vol 25, Springer, Berlin, (1996)

[251] D.W. Fisher, J. Phys. Chem. Solids 32, 2455 (1971).

[252] L.A. Grunes, R.D. Leapman, C.N. Wilker, R. Hoffmann, and A.B. Kunz, Phys. Rev. B 25, 7157 (1982).

[253] J. Ghijsen, L.H. Tjeng, J. Van Elp, H. Eskes, J. Westerink, G.A. Sawatzky, and M.T. Czyzyk, Phys. Rev. B 38, 11322 (1988).

[254] T.A. Albright, J.K. Burdett, M.-H. Whangbo, Orbital Interactions in Chemistry, Wiley, New York (1985).

[255] J.A. Tossel, D.J. Vaughan, and K.H. Johnson, Am. Mineral. 59, 319 (1974).

[256] L.F. Mattheis, Phys. Rev. 5, 190 (1972).

[257] M.A. Van Hove, W.H. Weinberg, and C.-M. Chan, Low Energy Electron Diffraction, Springer, Berlin (1986)

[258] M. Henzler and W. Göpel, Oberflächenphysik des Festkörpers, Teubner Studienbücher, Stuttgart (1994).

[259] M. Ritter, W. Ranke and W. Weiss, Phys. Rev. B 57, 7240 (1998).

[260] C.S. Fadley, M.A. Van Hove, Z. Hussain and A.P. Kaduwela, J. Electr. Spectr. Rel. Phen. 75, 273 (1995)

[261] W. Ranke, M. Ritter and W. Weiss, Phys. Rev. B 60, 1527 (1999).

[262] W. Kleber, Einführung in die Kristallographie, VEB Verlag Technik, Berlin (1979).

[263] E. Bauer and J.H. Van der Merwe, Phys. Rev. B 33, 3657 (1986).

[264] M. Bäumer, J. Libuda, and H.-J. Freund, in Chemisorption and Reactivity on Supported Clusters and Thin Films, R.M. Lambert and G. Pacchioni, (Eds.) Kluwer Acad. Press (1997), NATO ASI Ser. E Vol., p. 61-104.

[265] E. Pehlke, N. Moll, A. Kley and M. Scheffler, Appl. Phys. A 65, 525 (1997).

[266] S. Schwegmann, H. Over, V. De Renzi, and G. Ertl, Surf. Sci. 375, 91 (1995).

[267] C. Stampfl, S. Schwegmann, H. Over, M. Scheffler, and G. Ertl, Phys. Rev. Lett. 77, 3371 (1996).

[268] V.V. Roddatis, D.S. Su, C. Kuhrs, W. Ranke and R. Schlögl, Thin Solid Films, in press.

[269] M. Ritter, PhD thesis, Oberhofer, Berlin (1998).

[270] F.K. LeGoues, J. Tersoff, M.C. Reuter, M. Hammer, and R. Tromp, Appl. Phys. Lett. 67, 2317 (1995).

[271] I.M. Lifschitz and V.V. Slyozov, J. Phys. Chem. Sol. 19, 35 (1961).

[272] C. Wagner, Z. Elektrochem. 65, 581 (1961).

[273] B.K. Chakraverty, J. Phys. Chem. Sol. 28 (1967) 2401; J. Phys. Chem. Sol. 28, 2431 (1967).

[274] G. Wulff, Z. Kristallog. 34, 449 (1901).

[275] A. Zangwill, Physics at Surfaces, Cambridge University Press, Cambridge (1988).

[276] R.F.C. Farrow, G.R. Harp, R.F. Marks, T.A. Rabedeau, M.F. Toney, D. Weller and S.S.P. Parkin, J. Cryst. Growth 133, 47 (1993).

[277] Sh.K. Shaikhutdinov, M. Ritter, X.-G. Wang, H. Over and W. Weiss, Phys. Rev. B 60, 11062 (1999).

[278] Y. Joseph, G. Ketteler, C. Kuhrs, W. Ranke, W. Weiss, R. Schlögl, Phys. Chem. Chem. Phys. 3, 4141 (2001).

[279] A. Barbieri and M.A. Van Hove, Symmetrized Automated Tensor LEED package, available from M.A. Van Hove, Lawrence Berkeley Laboratory, 94720 Berkeley, USA.

[280] P.J. Rous, J.B. Pendry, D.K. Saldin, K. Heinz, K. Müller and N. Bickel, Phys. Rev. Lett. 57, 2951 (1986). 
[281] P.J. Rous and J.B. Pendry, Surf. Sci. 219, 355 (1989).

[282] P.J. Rous and J.B. Pendry, Comput. Phys. Comm. 54, 137 (1989); Comput. Phys. Comm. 54, 157 (1989).

[283] J.B. Pendry, J. Phys. C: Solid St. Phys. 13, 937 (1980).

[284] M. Ritter and W. Weiss, Surf. Sci. 432, 81 (1999).

[285] W. Moritz, available from W. Moritz, Institut für Kristallographie der Universität München, Theresienstr. 41, 80333 München, Germany.

[286] A. Yanase and N. Hamada, J. Phys. Soc. Jpn. 68, 1607 (1999).

[287] N.G. Condon, F.M. Leibsle, T. Parker, A.R. Lennie, D.J. Vaughan and G. Thornton, Phys. Rev. B 55, 15885 (1997).

[288] N.G. Condon, F.M. Leibsle, A.R. Lennie, P.W. Murray, T.M. Parker, D.J. Vaughan, and G. Thornton, Surf. Sci. 397, 278 (1998).

[289] M. Tsukada and T. Hoshini, J. Phys. Soc. Jpn. 51, 2562 (1982).

[290] J. Goniakowski and C. Noguera, Surf. Sci. 319, 68 (1994).

[291] J. Goniakowski and C. Noguera, Surf. Sci. 319, 81 (1994).

[292] M. Tsukada, E. Miyazaki, and H. Adachi, J. Phys. Soc. Jpn. 50, 3932 (1981).

[293] N.G. Condon, P.W. Murray, F.M. Leibsle, G. Thornton, A.R. Lennie and D.J. Vaughan, Surf. Sci. 310, L609 (1994).

[294] G. Ketteler, W. Weiss and W. Ranke, submitted to Surf. Rev. Lett.

[295] D. Frickel, Y. Arita, W. Ranke, to be published.

[296] X.-G. Wang, A. Chaka and M. Scheffler, Phys. Rev. Lett. 84, 3650 (2000).

[297] M.A. Nygren, D.H. Gay and R.A. Catlow, Surf. Sci. 380, 113 (1997).

[298] P.J. Eng, T.P. Trainor, G.E. Brown Jr., G.A. Waychunas, M. Newville, S.R. Sutton and M.L. Rivers, Science 288, 1029 (2000).

[299] J. Toofan and P.R. Watson, Surf. Sci. 401, 162 (1998).

[300] F. Jones, A.L. Rohl, J.B. Farrow and W. Van Bronswijk, Phys. Chem. Chem. Phys. 2, 3209 (2000).

[301] S. Thevuthasan, Y.J. Kim, S.I. Yi, S.A. Chambers, J. Morais, R. Denecke, C.S. Fadley, P. Liu, T. Kendelewicz and G.E. Brown Jr., Surf. Sci. 425, 276 (1999).

[302] S.C. Parker, N.H. De Leeuw and S.E. Redfern, Farad. Discuss. 114, 381 (1999).

[303] C.H. Rochester and S.A. Topham, J. Chem. Soc. Faraday Trans. I 75, 1073 (1979).

[304] C.F. Walters, K.F. McCarty, E.A. Soares and M.A. Van Hove, Surf. Sci. 464, L732 (2000).

[305] M.A. Henderson and S.A. Chambers, Surf. Sci. 449, 135 (2000).

[306] J. Ahn and J.W. Rabelais, Surf. Sci. 388, 121 (1997).

[307] J.M. Wittbrodt, W.L. Hase and H.B. Schlegel, J. Phys. Chem. 102, 6539 (1998).

[308] K.C. Hass, W.F. Schneider, A. Curioni and W. Andreoni, Science 282, 265 (1998).

[309] C.M. Eggleston, Am. Mineral. 84, 1061 (1999).

[310] R.J. Lad and V.E. Henrich, Surf. Sci. 193, 81 (1988).

[311] G. Thornton, priv. comm.

[312] Beilstein, 4th Edition, 3. Ergänzungswerk, Vol. V, p. 776.

[313] F.J. Himpsel, Adv. Phys. 32, 1 (1983).

[314] M. Getzlaff, priv. comm.

[315] A.J. Gordon and R.A. Ford, The Chemists Companion, Wiley, New York (1972).

[316] M.P. Seah and W.A. Dench, Surf. Interf. Anal. 1, 2 (1979).

[317] E. Lindholm, Faraday Discuss. Chem. Soc. (1972), p. 200.

[318] E. Lindholm, Faraday Discuss. Chem. Soc. (1972), p. 172.

[319] D.A. Shirley, in Photoemission in Solids I, M. Cardona and L. Ley, (Eds.), Topics in Applied Physics, Vol. 27, Springer, Berlin (1987), p. 165.

[320] D.W. Turner, A.D. Baker, C. Baker, and C.R. Brundle, Molecular Photoelectron Spectroscopy, Wiley-Interscience, New York (1970). 
[321] J.E. Demuth and D.E. Eastman, Phys. Rev. Lett. 32, 1123 (1974).

[322] K.Y. Yu, J.C. McMenamin, and W.E. Spicer, Surf. Sci. 50, 149 (1975).

[323] W. Huber, H.-P. Steinrück, T. Pache and D. Menzel, Surf. Sci. 217, 103 (1989).

[324] W.D. Grobmann and E.E. Koch, in Photoemission in Solids II, M. Cardona, L. Ley, (Eds.), Topics in Applied Physics, Vol. 27, Springer, Berlin (1978), p. 261.

[325] G.W. Robloff, H. Lüth, and W.D. Grobmann, Chem. Phys. Lett. 39, 493 (1976).

[326] G.L. Nyberg and N.V. Richardson, Surf. Sci. 85, 335 (1979).

[327] P. Hofmann, K. Horn, and A.M. Bradshaw, Surf. Sci. 105, L260 (1981).

[328] G.A. Somorjai, J. Phys. Chem. 94, 1013 (1990).

[329] S. Gokhale, P. Trischberger, D. Menzel, W. Widdra, H. Dröge, H.-P. Steinrück, U. Birkenheuer, U. Gutdeutsch, N. Rösch, J. Chem. Phys. 108, 5554 (1998).

[330] U. Birkenheuer, U. Gutdeutsch, and N. Rösch, Surf. Sci. 409, 213 (1998).

[331] K. Weiss, S. Gebert, M. Wühn, H. Wadepohl and Ch. Wöll, J. Vac. Sci. Technol. A 16, 1017 (1998).

[332] K. Weiss, J. Weckesser and Ch. Wöll, J. Mol. Struct. 458, 143 (1996).

[333] M. Wühn, Y. Joseph, P.S. Bagus, A. Niklewski, R. Püttner, S. Reiss, W. Weiss, M. Martins, G. Kaindl and Ch. Wöll, J. Phys. Chem. B 104, 7694 (2000).

[334] D. Pöss, W. Ranke, and K. Jacobi, Surf. Sci. 105, 77 (1981).

[335] J.F. Walsh, R. Davies, C.A. Muryn, G. Thornton, V.R. Dhanak, and K.C. Prince, Phys. Rev. B 48, 14749 (1993).

[336] H. Raza, P.L. Wincott, G. Thornton, R. Casanova, and A. Rodriguez, Surf. Sci. 404, 710 (1998).

[337] D. Schmeisser, F.J. Himpsel, G. Hollinger, B. Reihl, and K. Jacobi, Phys. Rev. B 27, 3279 (1983).

[338] S. Fölsch, A. Stock, and M. Henzler, Surf. Sci. 264, 65 (1992).

[339] K. Morokuma, J. Chem. Phys. 55, 1236 (1971).

[340] H. Umeyama and K. Morokuma, J. Am. Chem. Soc. 99, 1316 (1977).

[341] J.A. Connor, M. Considine, I.H. Hillier, and D. Briggs, J. Electr. Spectr. Rel. Phen. 12, 143 (1977).

[342] J.L. MacKay and V.E. Henrich, Phys. Rev. B 39, 6156 (1989).

[343] X.D. Peng and M.A. Barteau, Surf. Sci. 233, 283 (1990).

[344] J.M. McKay and V.E. Henrich, Phys. Rev. B 32, 6764 (1985).

[345] L. Giordano, J. Goniakowski and J. Suzanne, Phys. Rev. Lett. 81, 1271 (1998).

[346] P.J.D. Lindan, N.M. Harrison and M.J. Gillan, Phys. Rev. Lett. 80, 762 (1998).

[347] L.E. Firment and G.A. Somorjai, Surf. Sci. 84, 199 (1979).

[348] A. Fujishima and K. Honda, Nature 238, 37 (1972).

[349] M. Hendewerk, M. Salmeron, and G.A. Somorjai, Surf. Sci. 172, 544 (1986).

[350] V.A. Gercher and D.F. Cox, Surf. Sci. 322, 177 (1995).

[351] G.B. Fischer and B.A. Sexton, Phys. Rev. Lett. 44, 683 (1980).

[352] R.L. Kurtz and V.E. Henrich, Phys. Rev. B 26, 6682 (1982).

[353] W.J. Lo, W. Chung, and G.A. Somorjai, Surf. Sci. 71, 199 (1978).

[354] N.B. Brooks, F.M. Quinn, and G. Thornton, Vacuum 38, 405 (1988).

[355] R.L. Kurtz, R. Stockbauer, T.E. Madey, E. Roman, and J.L. De Segovia, Surf. Sci. 218, 178 (1989).

[356] P.B. Smith and S.L. Bernasek, Surf. Sci. 188, 241 (1987).

[357] R.J. Speedy, P.G. Debenetti, R.S. Smith, C. Huang and B.D. Kay, J. Chem. Phys. 105, 240 (1996).

[358] Y. Joseph, M. Wühn, A. Niklewski, W. Ranke, W. Weiss, Ch. Wöll and R. Schlögl, Phys. Chem. Chem. Phys. 2, 5314 (2000).

[359] P. Scharfenberg, B. Rozsondai, I. Hargittai, Z. Naturforsch. 35 a, 431 (1980). 
[360] J.C. Cochran, K. Hagen, G. Paulen, Q. Shen, S. Tom, M. Traetteberg, C. Wells, J. Mol. Struc. 413-414,313 (1997).

[361] M. Henzler, in Electron Spectroscopy for Surface Analysis, H. Ibach, (Ed.), Springer, Berlin (1979).

[362] W. Weiss, D. Schmeisser and W. Göpel, Surf. Sci. 207, 401 (1989).

[363] Sh.K. Shaikhutdinov, Y. Joseph, C. Kuhrs, W. Ranke, W.Weiss, Farad. Disc. 114, 363 (1999).

[364] D. Zscherpel, W. Ranke, W. Weiss, R. Schlögl, J. Chem. Phys. 108, 9506 (1998).

[365] C. Kuhrs, Y. Arita, W. Weiss, W. Ranke and R. Schlögl, Topics in Catal. 14, 111 (2001).

[366] C. Kuhrs, PhD thesis, Logos, Berlin (2000).

[367] W. Weiss, D. Zscherpel, R. Schlögl, Catal. Lett. 52, 215 (1998). 